\title{
SENSOR FUSION FOR CLOSED-LOOP \\ CONTROL OF UPPER-LIMB PROSTHESES
}

\section{UNIVERSITÄTSMEDIZIN $=\mathbf{M G}$
GÖTTINGEN}

\author{
Marković Marko \\ Universitätsmedizin Göttingen \\ Bernstein Center for Computational Neuroscience \\ Institute of Neurorehabilitation Systems \\ Universitätsmedizin Göttingen
}

This dissertation is submitted for the degree of Dr. sc. hum.

February 2016 

Supervisor: Prof. Dr. Dr. Dario Farina

Institute for Neurorehabilitation Systems

Universitätsmedizin

Georg-August-Universität Göttingen

Second Supervisor: Dr. Bernhard Graimann

Department of Translational Research and Knowledge Management

Otto Bock HealthCare GmbH

Duderstadt

Third Supervisor: Prof. Dr. Ulrich Sax

Department of Medical Informatics

Universitätsmedizin

Georg-August-Universität Göttingen 


\section{DECLARATION}

This dissertation is the result of my own work and includes no outcome of work done in collaboration except where specifically indicated in the text. It has not been previously submitted, in part or whole, to any university of institution for any degree, diploma, or other qualification.

Signed: Mopprer Maphlut

Date: $\quad 12.02 .2016$

Marković Marko

Göttingen 


\begin{abstract}
This thesis addresses open challenges in the field of myoelectrically controlled upper limb prostheses. These challenges include the inherently low bandwidth of the myoelectric control channel that makes the current control interfaces limited and unintuitive for the user, especially when controlling modern multi-function prostheses, as well as the lack of somatosensory feedback that would allow the user to better perceive the state of his/her prosthesis. This thesis aims at addressing these challenges by designing novel man-machine-interfaces, based on the latest sensing and automatic control technologies, to provide improved operation and perception of the prosthetic device. To this end, the thesis comprises introductory chapters that describe the state of the art in the field, the aim of the thesis and the used methodology, as well as four peerreviewed journal publications presenting the novel feedforward and feedback methods.

In the first two studies, I proposed and evaluated a novel system for prosthesis control based on sensor-fusion. In the classic approach, the user has the responsibility of generating all the command signals, while the prosthesis controller operates as a decoder, acquiring the signals and decoding user intention. In the novel framework, proposed here, the prosthesis is enhanced with advanced sensing and autonomous decision-making, becoming thereby an intelligent agent assisting the user. The inspiration for this novel approach comes from the modern-day autonomous robotic systems that utilize a variety of multimodal sensors and data processing methods in order to perceive and interpret the environment. In the present work, the prosthetic hand was equipped with computer vision and inertial sensing, and this information was used by the prosthesis controller to provide an additional, artificial-intelligence processing layer. This component analyzed the usage-context (environment, user and prosthesis) and, based on this, automatically controlled the hand preshape and orientation, thereby supporting the user in operating the prosthesis functions. The overall control loop is therefore simplified for the user because the sensor-fusion controller takes over the part of the inherent control complexity by adjusting the prosthesis parameters automatically. The user only provides high-level commands (e.g., grasp an object) that can be delivered robustly through a simple two-channel myoelectric interface.

In the second two studies, I introduced a versatile development framework for evaluating a variety of feedback interfaces. The framework comprises a library of
\end{abstract}


components implementing specific elements of a generic closed-loop prosthesis control system, from control inputs to the feedback interfaces. The framework operates in realtime and allows fast prototyping and testing. This has been used to develop and evaluate a novel biofeedback paradigm that closes the loop by feeding the myoelectric control signals (prosthesis input) back to the user. This is a novel approach with respect to the classic methods in literature, in which the feedback variables were the prostheses outputs (e.g., grasping force, joint angle). Due to the nature of the prosthesis control interface, in which the prosthesis reaction is proportional to the user's myoelectric commands, this paradigm allows for predictive and robust control in comparison to the state-of-the-art approaches. For example, the user can exploit the biofeedback to modulate his/her command to the prosthesis during closing, so that desired grasping force is generated, after contact (predictive force control). Finally, a practical biofeedback implementation that utilizes augmented-reality and wearable see-through display embedded in Google Glass is also presented.

In conclusion, by innovating both feedforward as well as feedback interfaces with new functionalities, this thesis advances the overall development of the modern manmachine-interfaces used for prosthesis control. This research can lead to effective and user friendly methods for control of advanced modern-day prostheses (e.g., dexterous hands, full arms), which in return could improve the utility and facilitate wider acceptance of these systems in daily life. 


\section{ACKNOWLEDGEMENTS}

Hereby I would like to acknowledge all my $\mathrm{PhD}$ supervisors and a colleagues of mine at University of Göttingen, Dr. Meike Schweisfurth and Dr. Strahinja Došen, for an excellent and continuous scientific guidance and support. 


\section{CONTENTS}

1 INTRODUCTION...................................................................................................1

1.1 DEVELOPMENT OF PROSTHETIC HANDS: HISTORICAL PERSPECTIVE .......................... 2

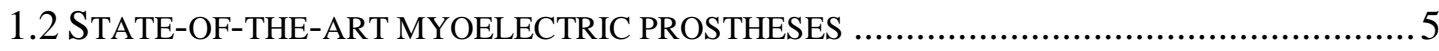

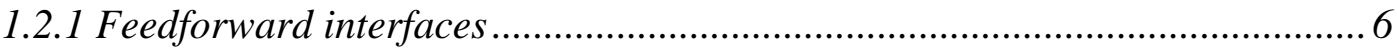

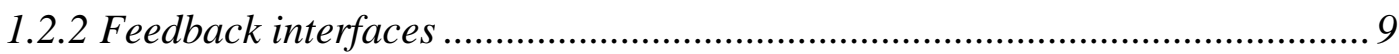

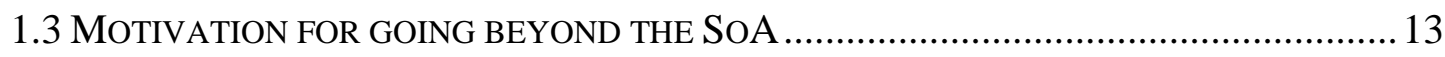

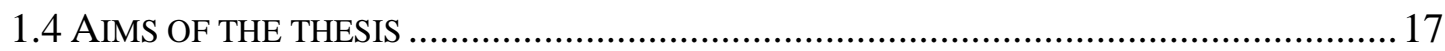

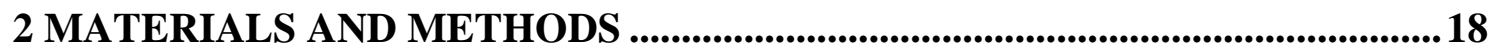

2.1 AdDRESSING Prosthesis CONTROL: SENSOR-FUSION CONCEPT ........................... 18

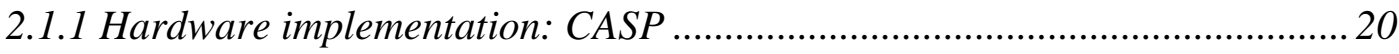

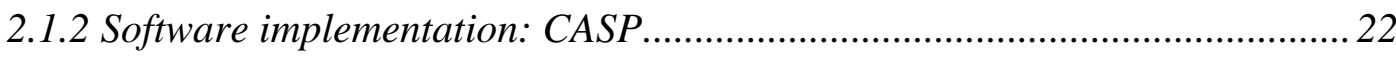

2.1.3 Experimental setup and evaluation: CASP .............................................2 26

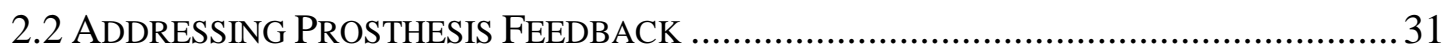

2.2.1 Closed loop development framework …..................................................... 32

2.2.2 A novel feedback concept: Biofeedback ....................................................... 36

2.2.3 Experimental setup and evaluation: Biofeedback ........................................ 36

2.2.4 Practical implementation of the Biofeedback: Google Glass........................ 38

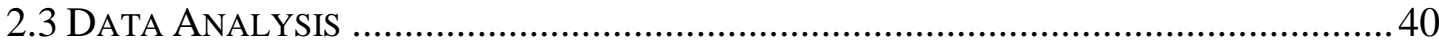

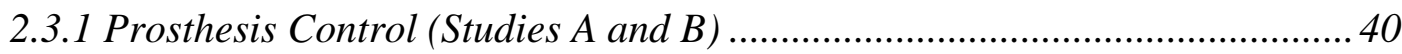

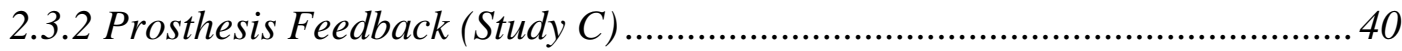

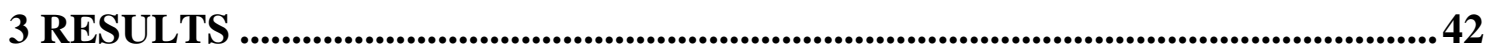

3.1 Prosthesis Control (StUdies A AND B) .................................................... 42

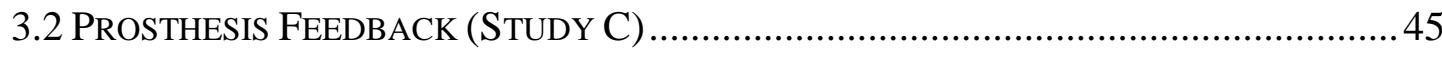

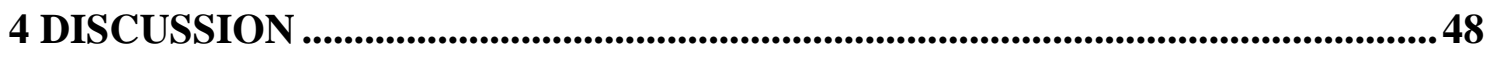

4.1 Prosthesis Control (CASP, StUdies A AND B) ................................................. 48

4.2 Prosthesis FEEdBACK (CLF, BIOFEEDBACK, StUdy C) ...................................50 


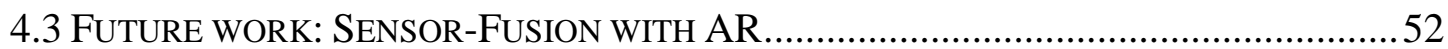

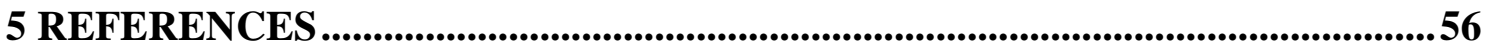

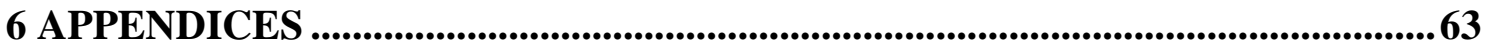




\section{LIST OF TABLES}

Table 2.1: The key-differences between the previous and current system iteration are summed up in the table below and are divided into two sections: features and technical specifications.

Table 2.2: Summary of the experimental evaluations performed in Studies A and B. (*) For more information, consult Table 2.1. (**) AR feedback is discussed separately in Appendix 1, [71](***) The outcome measures have a different abbreviation in original study.

Table 2.3: Summary of the experimental evaluations performed in Study C .37 


\section{LIST OF FIGURES}

Figure 1.1: Some compensatory strategies utilized during typical ADL (drinking, driving) by a bilateral (a) and a unilateral (b) amputee.

Figure 1.2: Overview of the prosthetic devices: (a) transradial cosmesis, (b) transradial body-powered prosthesis with a harness (image adapted from Chorost, 2012), (c) transradial myoelectric prosthesis (image adapted from Encyclopædia Britannica, Inc.), and (d) hybrid transhumeral prosthesis combining a harness for hand opening/closing and myoelectric control for elbow flexion/extension.

Figure 1.3: Prostheses recommendations based on the amputation level: ++: best option, +: good option, o: suitable option, -: unsuitable option. Image adapted from [10]

Figure 1.4: Commercially available myoelectric prostheses: a) Multi-finger articulated i-limb ultra (Touch Bionics Inc., UK, [11]), b) Michelangelo Hand with an active wrist and two-grip patterns (Otto Bock HealthCare GmbH, Germany, [12]), and c) Utah Arm 3 with an active elbow and a simple gripper (Motion Control Inc.,USA, [13])

Figure 1.5: SoA (commercial) direct control system for multi-DoF prosthesis. The user controls each DoF sequentially via two bipolar sEMG electrodes placed on the residual stump. The prosthesis will not react as long as the muscle activity is below the two predefined thresholds (T1, T2). Once the threshold is reached, the prosthesis starts the movement proportionally to the strength of the muscle contraction. The DoF switching is triggered by strongly co-contracting the two muscles for a short period of time.

Figure 1.6: Targeted muscle reinnervation procedure. Image adapted from [23]. .............................. 8

Figure 1.7: SoA (research) sEMG pattern-recognition system for multi-DoF prosthesis control. Image adapted from [32].

Figure 1.8: Three possible feedback information pathways in the context of myoelectric prostheses. Different colors correspond to different pathways (A, B, C). Pathway A is related to sensory information that is directly fed back to the CNS (e.g., visual and auditory feedback); Pathway B to the information that is conveyed to functional sensory motor systems invasively or noninvasively; Pathway $\mathrm{C}$ is related to the intrinsic feedback. Image adapted from [35].

Figure 1.9: a) Mechanical- and b) thermal-tactor used for modality matched feedback. Images adapted from [37].

Figure 1.10: a) The SoA C2-Tactor can be used for vibro-tactile stimulation (Engineering Acoustics, Inc, Florida, USA [42]); b) a disposable surface electrode typically used for electro-tactile stimulation (Spes Medica Genoa, Italy))

Figure 1.11: Amputation paradox: the higher the amputation level, the less muscles are available to control the increasing number of prosthesis functions.

Figure 1.12: Diversity of choices when designing a typical CLS. Typical CLS needs at least one component from each of the columns (a), b), c), d), and e)). The components can be combined almost arbitrary which renders a virtually infinite number of CLS that can be designed.

Figure 2.1: Comparison between a) SoA myoelectric control system and b) the novel context- and useraware prosthesis controller (CASP). With CASP the prosthesis is controlled semi-autonomously by fusing signals acquired from the user and from an array of additional external sensors; the CASP controller thus reduces the overall user throughput by taking care of the increased prosthesis complexity (note how the line thickens change between the user, the controller and the prosthesis from a) to b))

Figure 2.2: a) The relevant components of the CASP prototype are shown. b) The CASP system prototype utilized by an amputee to grasp a book. Image adapted from [72].

Figure 2.3: Conceptual scheme of the algorithm driving the CASP system. The central feature of the system is the sensor fusion, which allows for context-dependent reactive prosthesis control. List of abbreviations: Hand preshape and orientation (HPO), object of interest (OI), current prosthesis rotation (current_Rot), rotation of the selected hand posture (HPO(i)_Rot).

Figure 2.4: Experimental setup/task used for evaluating both iterations of the CASP system. The EMG electrode placement is denoted with "e". The dashed circle represents where the object should be 
released. Experimental task (1-5) is denoted in blue. (*) The IMU (hexagon) was not implemented in the first system iteration.

Figure 2.5: a) The structure of the closed-loop development framework (CLF). The implemented components are divided into eight different libraries organized into separate folders. Each component follows the same structural organization consisting of a component-model (.slx) and dependencies folders. Custom-designed CLSs are saved in a separate folder tree (test bench models). b) CLF integrated in Simulink Library Browser. In order to use it, the user needs to navigate to the desired component and simply drag and drop it into his model. . .33

Figure 2.6: An exemplary closed-loop system setup used for evaluating the human performance in steering the prosthesis' force while utilizing tactile feedback. The flash symbols indicates that the additional, component-specific, settings open once the annotated component is double-clicked (customization). Each component has an intuitive icon and displays its most important parameters in red (transparency of operation). The overall CLS model execution is configured within a single block. The model will automatically compile and execute as soon as the START button is double clicked (centralized high-level system management). The data logging block logs the model settings automatically (e.g., component and model parameters) as well as the user-selected signals.

Figure 2.7: Fundamental differences between EMG biofeedback (bioFB) and force feedback (forceFB) in the context of prosthesis control. During forceFB, the generated grasping force is transmitted to the user only after touch onset, whereas during bioFB the user receives information about the generated EMG online even before the prosthesis reacts. Image adapted from [74].

Figure 2.8: Visual scene shown to the subjects during Study C including a snapshot of the screen (a) before contact and (b) after contact. Horizontal bars, red for the flexor and blue for the extensor, showed a continuous feedback about the current level of muscle activity (prosthesis control signals). The semi-transparent blue bar indicated the hand grasping force and the green vertical line the target force level. During routine grasping, the target force was stationary, whereas in the force steering task, it was moving according to the time profile of a reference force trajectory. Note that the biofeedback was shown only during the respective feedback scenario. Image adapted from [74].

Figure 2.9: Biofeedback implementation on the Google Glass. The Glass connects to the Michelangelo prosthesis via BT and renders the acquired feedback on the OHDM. The user can switch between different representations by performing a scroll gesture on the touchpad.

Figure 3.1: Study A: a) Summary results for the CASP preshape success rate (PSR). b) Task accomplishment/failure rate (TAR/SFR) for the two operation modes: without (AUTO) and with manual user control (SEMI). Statistically significant differences are denoted by a star $(*, \mathrm{p}<0.05)$. Image adapted from [71].

Figure 3.2: Study B: a) Summary results for the average time to grasp (TTG) an object across conditions (MAN1-3, CASP) and experimental sessions (training, evaluation). The statistically significant differences are denoted by a star $(*, p<0.05)$; the symbol ' $\mathrm{C}^{\text {' }}$ indicates that the difference exists across all conditions that were performed within the same experimental session. b) 3D model showing the arm positions recorded shortly before the object was grasped. An object placed horizontally (left) and vertically (right) was grasped using MAN1 and CASP control schemes. Image adapted from [72].

Figure 3.3:Sudy C: a) Routine grasping task performance in two feedback conditions (forceFB and bioFB) and at three target force levels (30, 50, and 70\%). Boxplots depict the median (red line), interquartile range (blue box), maximal/minimal values (whiskers) and outliers (red crosses). Dashed gray lines are the target force levels. b) Force-tracking task performance. The toot mean square tracking error is given for two conditions (forceFB, and bioFB). Statistically significant differences are denoted by a star $(*, p<0.05)$. Image adapted from [74]. .46

Figure 4.1: The envisioned future of upper limb prostheses. The user wears an array of devices that communicate wirelessly in order to fuse and process multimodal sensory information. The smartphone acts as the central processing unit that communicates with a cloud in order to perform computationally intensive operations or update the map of the user's environment. The smartglasses integrate the RGB-D camera together with the see-through AR display thus acting as an artificial exteroception and feedback interface. The smartwatch/smartband is worn around the unaffected arm and provides rudimentary feedback to the user (notifications) as well as the artificial proprioception to the overall control system. 


\section{LIST OF ABBREVIATIONS AND ACRONYMS}

ADL Activities of the daily living

AR Augmented reality

AUTO Fully autonomous control

BT Bluetooth

CLF Closed-loop development framework

CLS Closed-loop system

DoF Degree-of-freedom

(s)EMG (surface)Electromyography

FoV Field-of-view

GUI Graphical user interface

HPO Hand preshape and orientation

IMU Inertial measurement unit

MAV Mean-average value

MAN Fully manual control

MMI Man Machine Interface

OEE Orientation estimation error

OHMD Optical head mounted display

OI Object of Interest

PSR Preshape success rate

RGB-D Red, green, blue (i.e., color) and depth (i.e., grayscale) image

RMS Root-mean square

RMSE Root mean square error 
SEE Size estimation error

SEMI Semi-autonomous control

SFR System-induced task failure rate

SoA State-of-the-art

TAR Task accomplishment rate

TMR Target muscle reinnervation

TTG Time to grasp 


\section{LIST OF APPENDICES}

Appendix 1: Stereovision and augmented reality for closed-loop control of grasping in hand prostheses 65 Appendix 2: Sensor fusion and computer vision for context-aware control of a multi degree-of-freedom

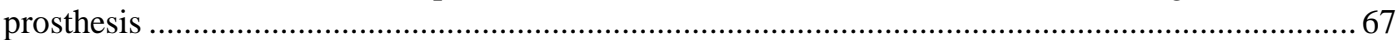

Appendix 3: Sensory feedback in prosthetics: a standardized test bench for closed-loop control .............. 69 Appendix 4: EMG Biofeedback for online predictive control of grasping force in a myoelectric prosthesis 



\section{INTRODUCTION}

Hands are an essential part of our body. They are a truly outstanding dexterous tool, essential for our interaction and perception of the world around us [1]. Therefore, it is of no surprise that their sudden loss, which is usually a consequence of a trauma caused by industrial/motor vehicle accident or equally often in current day and age battlefield injury, presents a significant impact on a person's life. It is estimated that in the United States only, there are approximately 541,000 people with an upper limb amputation [2]. Observed annually in the USA there are 18,496 upper limb amputations per year, with $99.7 \%$ of them accounting for unilateral and the rest for bilateral amputations. The vast majority of them (91\%) occur at below hand-wrist level (wrist disarticulation), and are usually triggered by trauma (68.6\%) or tumor (23.9\%) [3]. Additionally, a congenital limb deficiency that occurs in $0.02 \%$ to $0.07 \%$ of births [4] has a prevalence rate of $50 \%$ in upper limbs [3]. Worldwide there are millions of people whose lives are affected by the upper limb loss.

The loss of an upper limb is not necessarily a critically limiting factor in a person's life, especially when considering the overall versatility of people when coping with the disability in the context of activities of daily living (ADL, see Figure 1.1). Nevertheless, it is considered that, in the long run, lost functions are best to be replaced with an appropriate assistive device due to the imminent development of compensatory strategies that might have a negative impact on a person's overall well-being. In this chapter the reader will be introduced to the assistive devices, technologies, and methods that are currently at the disposal for treating limb loss. 
Figure 1.1: Some compensatory strategies utilized during typical ADL (drinking, driving) by a bilateral ( $a$ ) and a unilateral (b) amputee.
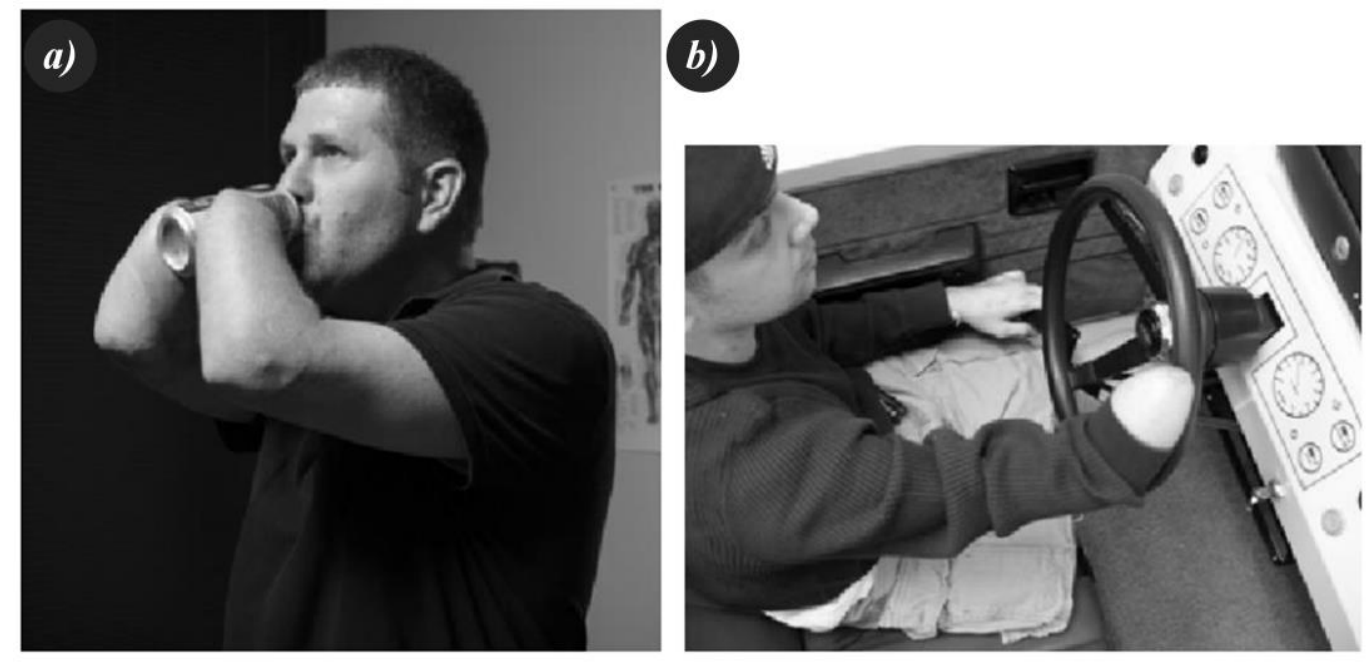

\subsection{Development of prosthetic hands: historical perspective}

Based on their function and interface, upper limb prostheses can be divided into four categories (Figure 1.2):

a) Passive: Limited or no functionality (often referred to as "cosmesis");

b) Active, body-powered: Operated and actuated directly by the user via a wearable harness;

c) Active, externally-powered: Actuated via a motor, operated via a myoelectric interface (often referred to as myoelectric prosthesis);

d) Active, hybrid: Combined body- and externally-powered systems;

Historically, the usage of passive prosthetic devices dates back to $300 \mathrm{BC}$ in old Egypt [5]. Even though very limited in functionality, they can act as support and potentially prevent some postural problems. To this date hand cosmeses are a preferred choice of many amputees due to their overall simplicity and exceptional appearance [6]. The first passive prostheses with some albeit limited dexterity appeared in the middle age and as such they were most often used as part of the bodily armor. For this reason, they were referred to as "knight hands". These prostheses used a system of springs, which could be adjusted by the contralateral hand in order to control one or more finger joints. Interestingly, Löffler [5] also reports that there were around 50 of these passive hands, ranging from simple to complex, used in $16^{\text {th }}$ and $17^{\text {th }}$ century.

The active, body powered prostheses appeared shortly after the outbreak of the $1^{\text {st }}$ World War with the first patent being submitted and accepted already in 1912 [7]. The 
body-powered systems are usually cable-driven meaning that the prosthesis functions are steered via a Bowden cable that is attached to the harness worn by the user, usually on the contralateral side. In a typical usage scenario (shown in Figure 1.2b) the user extends the arm or flexes his shoulder and the harness pulls the attached cable (1). As the cable tightens, it opens the hook (2); reversing the move closes the hook (3). In case of a multi degree-of-freedom (DoF) prosthesis, a manual switch is used to operate the DoFs sequentially (e.g., hand open/close or wrist pronation/supination). The critical downside of this system is that it actually fosters usage of over-compensation strategies on the unaffected side (e.g., pronounced shoulder disarticulation); also, the overall visual appeal of these prostheses might suffer due to the cumbersome cable/harness system. Nevertheless, body-powered prostheses are still the preferred choice of many amputees since they present a good compromise between usability, ease-of-use, and overall robustness.

Figure 1.2: Overview of the prosthetic devices: (a) transradial cosmesis, (b) transradial body-powered prosthesis with a harness (image adapted from Chorost, 2012), (c) transradial myoelectric prosthesis (image adapted from Encyclopadia Britannica, Inc.), and $(d)$ hybrid transhumeral prosthesis combining a harness for hand opening/closing and myoelectric control for elbow flexion/extension.

\section{a)}

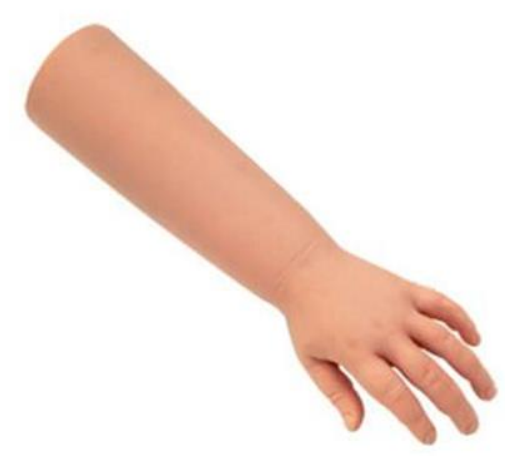

c)

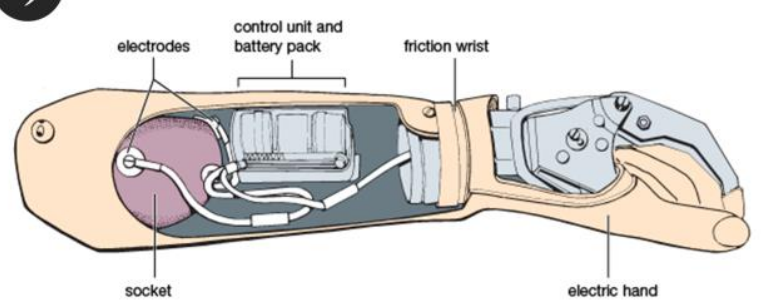

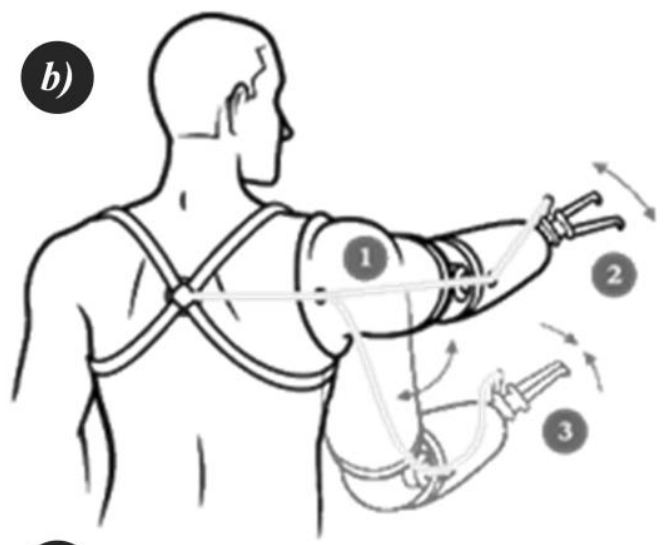

d)

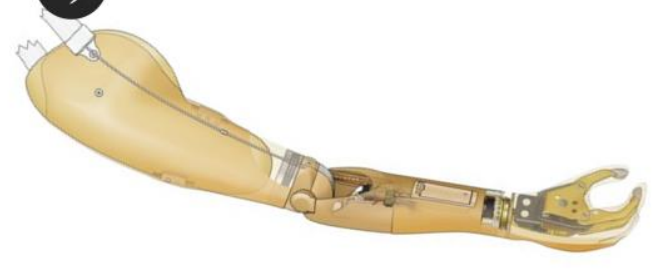

Externally powered prostheses employ specifically processed surface signals acquired from the residual limb muscles in order to control the amount of current in the prosthesis' actuator (Figure 1.2c). These prostheses are in application since approximately 50 years [8] and their control interface is based on a basic physiological 
phenomenon: generation of the muscle action potentials. During a muscle contraction, electric potentials are generated by muscle fibers and produce extracellular field potentials, also called electromyogram (EMG), which can be measured with electrodes on the skin surface [9]. The amplitude of the surface EMG (sEMG) signal depends on the number of active muscles and the rate at which they are activated [9]. Myoelectric prostheses are considered an optimal fit for transradial amputees, since the remaining wrist and finger flexion or extension muscles can be used to control the prosthesis opening or closing function in a rather intuitive manner. Myoelectric prostheses, similarly to the body-powered, can act on multiple DoFs with switching systems. Moreover, the two prosthetic types, externally and body powered ones can be even combined together in a hybrid system (Figure 1.2d).

The four prosthesis types (cosmesis, body-powered, myoelectric, and hybrid) are the choices currently available to patients. The choice most suited to a specific user depends on subjective preferences, level of amputation, age, medical implications, social environment, etc. Nevertheless, as a general rule, cosmetic prostheses are recommended for partial hand/finger or shoulder amputations, myoelectric prostheses for transradial and above elbow amputations, while the body powered prostheses fit best users who suffer from around elbow amputations (Figure 1.3).

Figure 1.3: Prostheses recommendations based on the amputation level: ++: best option, +: good option, o: suitable option, -: unsuitable option. Image adapted from [10]

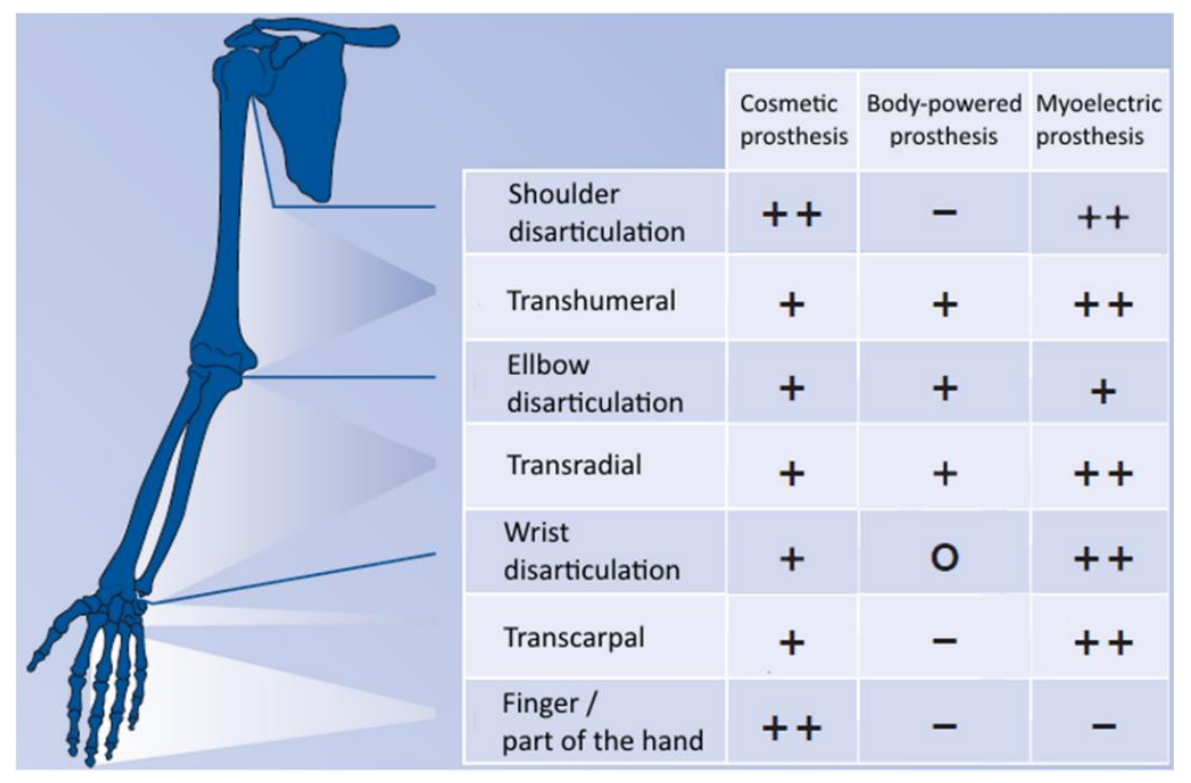

In the following chapter, the reader will be introduced to state-of-the-art (SoA) myoelectric prostheses with a specifically critical retrospective on their development, 
functionality, control, and feedback interfaces.

\subsection{State-of-the-art myoelectric prostheses}

Technologically, myoelectric prostheses present the most complex solution for replacing the lost limb with a variety of functionalities, ranging from simple grippers to highly dexterous (i.e., multi-DoF) systems (Figure 1.4).

Figure 1.4: Commercially available myoelectric prostheses: a) Multi-finger articulated i-limb ultra (Touch Bionics Inc., UK, [11]), b) Michelangelo Hand with an active wrist and two-grip patterns (Otto Bock HealthCare GmbH, Germany, [12]), and c) Utah Arm 3 with an active elbow and a simple gripper (Motion Control Inc., USA, [13])

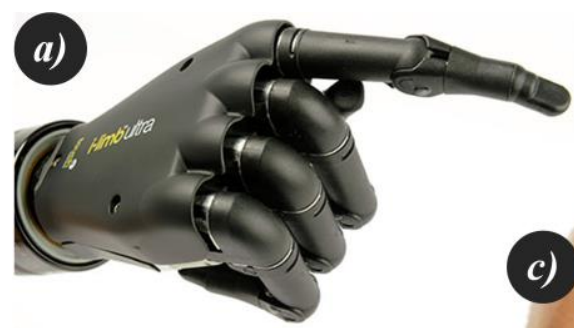

b)

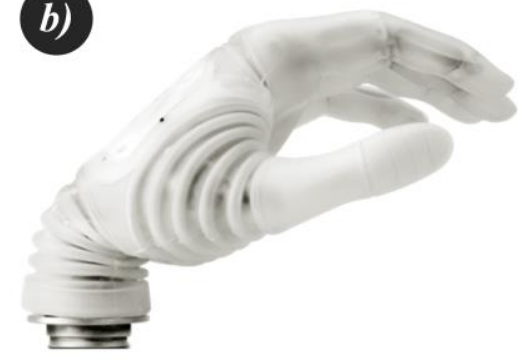

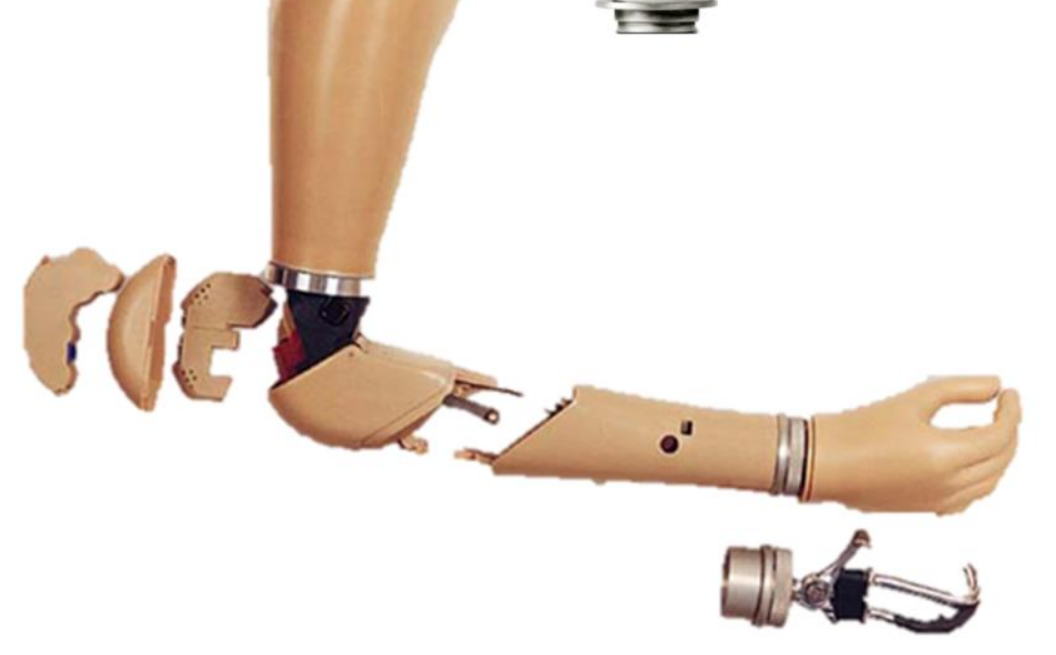

Since the prostheses are designed to replace the lost function, they cannot be extrapolated by the adopted man-machine-interface (MMI) that serves as the interaction between the prosthetic device and its user. This interaction should ideally be proficient in both directions, as we rely on both feedforward and feedback pathways in order to act in and manipulate our environment [14], [15]. Feed-forward control is based on internal models through which we anticipate a movement or upcoming interaction with an object. Internal models are built through previous sensorimotor experiences and are updated by the integration of new sensory information (i.e. sensory feedback) that we continuously receive [16], [17], [18]. Each specific behavior or movement pattern relies on its own internal model that develops from repeated practice. For example, the internal model of a power grip allows us to grasp an object as economically as possible, 
i.e. to scale the grip force according to the weight and surface of the object, so that the effort is minimized yet slips are avoided. Therefore, feedforward and feedback pathways build a closed-loop that enables precise and effortless motor control. This is the reason why, in the context of a myoelectric prosthesis control, the overall quality and performance of the employed MMI can be a decisive factor for its acceptance and utility. Ideally, a good MMI could be seen as a bidirectional communication channel through which the user can simultaneously and with great ease control (feedforward channel) and perceive (feedback channel) the prosthesis.

\subsubsection{Feedforward interfaces}

In this chapter, the reader will be introduced to the state of the art control interfaces that are used in commercial as well as in research-based myoelectric prosthetic systems.

\subsubsection{Commercial SoA}

Currently, the most commonly used commercial myoelectric interfaces are designed as two-site electrode systems that implement direct proportional control. In these systems the two electrode systems are positioned over a pair of antagonist muscles (e.g., over the wrist flexor and extensor muscle groups in case of a transradial amputation), which are then used by the amputee to directly modulate the prosthesis' movement velocity. The implementation of such system can be summarized as follows (Figure 1.5) [19]:

- The sEMG is pre-processed (i.e., amplified and filtered) in several stages via special electronics. Then, it is fed into an analog rectifier, or a similar circuit, that calculates its amplitude, either as mean-average value (MAV) or root-mean square (RMS) value or low-pass filtering;

- When the EMG amplitude is above a predefined threshold, the prosthesis moves with a velocity proportional to muscle activity - the higher the amplitude (i.e., the muscle activation), the faster the prosthesis moves. The movement direction is determined by the "larger-signal-wins" strategy: the channel with greater amplitude dictates the direction (e.g., hand open vs. close);

- Finally, in the case of a multi-DoF prosthesis, the control over each DoF is performed sequentially. The user needs to switch between, e.g., hand opening/closing and a wrist pronation/supination function via a pre-defined trigger (i.e., muscle pattern). To achieve this switching, quickly timed and strong muscle cocontractions or bursts are found to be particularly convenient; 
Figure 1.5: SoA (commercial) direct control system for multi-DoF prosthesis. The user controls each DoF sequentially via two bipolar sEMG electrodes placed on the residual stump. The prosthesis will not react as long as the muscle activity is below the two predefined thresholds $(T 1, T 2)$. Once the threshold is reached, the prosthesis starts the movement proportionally to the strength of the muscle contraction. The DoF switching is triggered by strongly co-contracting the two muscles for a short period of time.

sEMG Electrode 1 (wrist extensors)

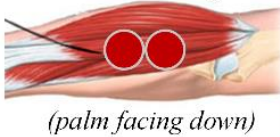
(palm facing down)

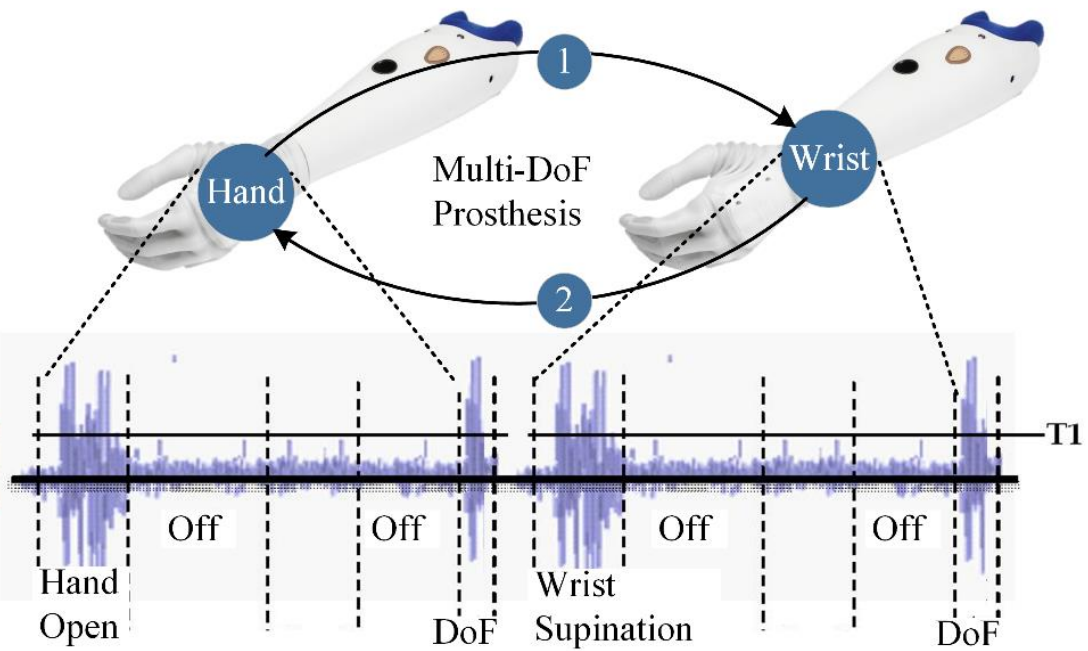

DoF Supination Switch

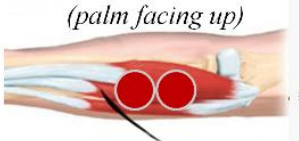

sEMG Electrode 2 (wrist flexors)

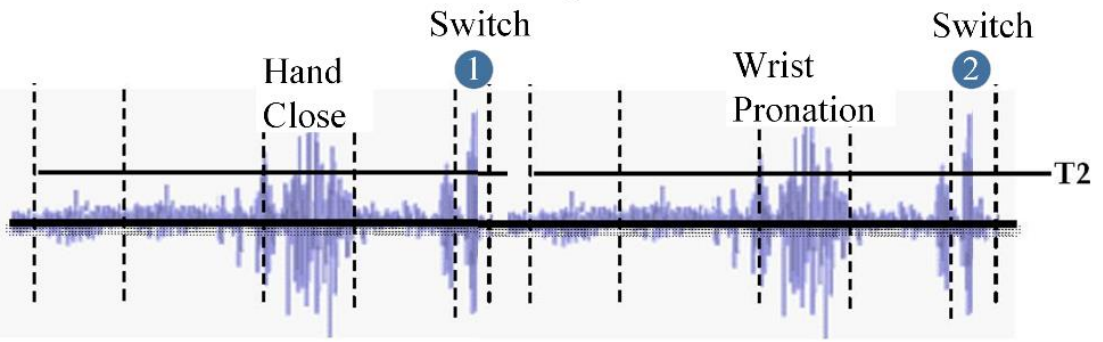

This concept of myoelectric prosthesis control exists for well over 70 years [20] and has been the first one to be used in commercial myoelectric prostheses starting from the late 60's [21]. Depending on the user needs and capabilities, the direct control systems are several: one-site control ("cookie crusher"), digital control, multi-level control, etc. [19].

Direct control of DoFs is particularly effective following target muscle reinnervation (TMR) [22]. TMR is a surgical intervention that transfers the residual nerves from an amputated limb onto alternative muscle groups (e.g., plexus muscles) that are not biomechanically relevant since they are no longer attached to the missing arm. During this procedure, target muscles are denervated so that they can be reinnervated by the residual arm nerves which are surgically placed close to their target nerve or muscle. After the post-op recovery process, the reinnervated muscles can serve as biological amplifiers of the amputated nerve motor commands (Figure 1.6). If the outcome was successful the amputee will be able to control directly, proportionally, and simultaneously up to 3-DoF's in an intuitive manner because the sites of EMG detection 
are well separated spatially.

Figure 1.6: Targeted muscle reinnervation procedure. Image adapted from [23].

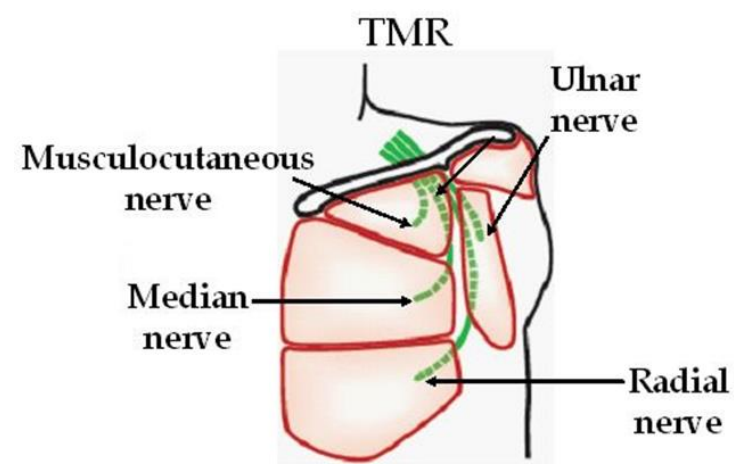

\subsubsection{Research SoA}

Opposed to the commercially available, the academic SoA is virtually unconstrained in terms of its interface size and complexity. This is because, in the academic context, the practical implementation and device costs often play a secondary role in comparison to the possibly added functionality. Therefore, the feedforward MMIs developed in academic environment often use powerful machine learning algorithms. These algorithms are able to infer complex prosthesis movement by analyzing the muscle activation patterns in the residual limb. They have been in use since around 35 years [24] but they have gained popularity only with the development of powerful microprocessors in the early 90s. Since then they constitute the primarily used MMI in research. As for direct control, there is a great variety of machine learning interfaces. They can be implemented in a virtually infinite number of ways: artificial-neuralnetworks/fuzzy logic [25], [26], [27] pattern-recognition [28], [29], [23] or regression [30], [31] based systems are just few of many existing examples in the literature. In most general sense, the pattern-recognition approach can be summed up as follows (Figure 1.7, [32]):

- The sEMG is acquired from several sites (e.g., usually from 6 to 12) distributed uniformly around the residual stump;

- Time and/or frequency features [25] are extracted over a sliding time window from each of the sEMG channel resulting in $\mathrm{L} \times \mathrm{C}$ feature matrix (where $\mathrm{L}$ represents the number of extracted features and $\mathrm{C}$ the number of channels). For performance reasons the time windows are usually $150 \mathrm{~ms}$ long with $50 \%$ overlap;

- Thus extracted, the feature matrix is fed into a machine-learning algorithm. 
Based on the prior knowledge, the machine-learning algorithm assigns the feature matrix into pre-designated movement classes (e.g., hand open, wrist supination, etc.) and moves the prosthesis accordingly. Interestingly, the proportionality of control is usually lost in this process, and additional cues need to be used in order for it to be inferred.

- It should be noted that the machine-learning algorithm must be trained before it is placed into use. The training process complexity will largely depend on the number of utilized classes, which is again correlated to the overall complexity of the prosthetic system;

Figure 1.7: SoA (research) sEMG pattern-recognition system for multi-DoF prosthesis control. Image adapted from [32].

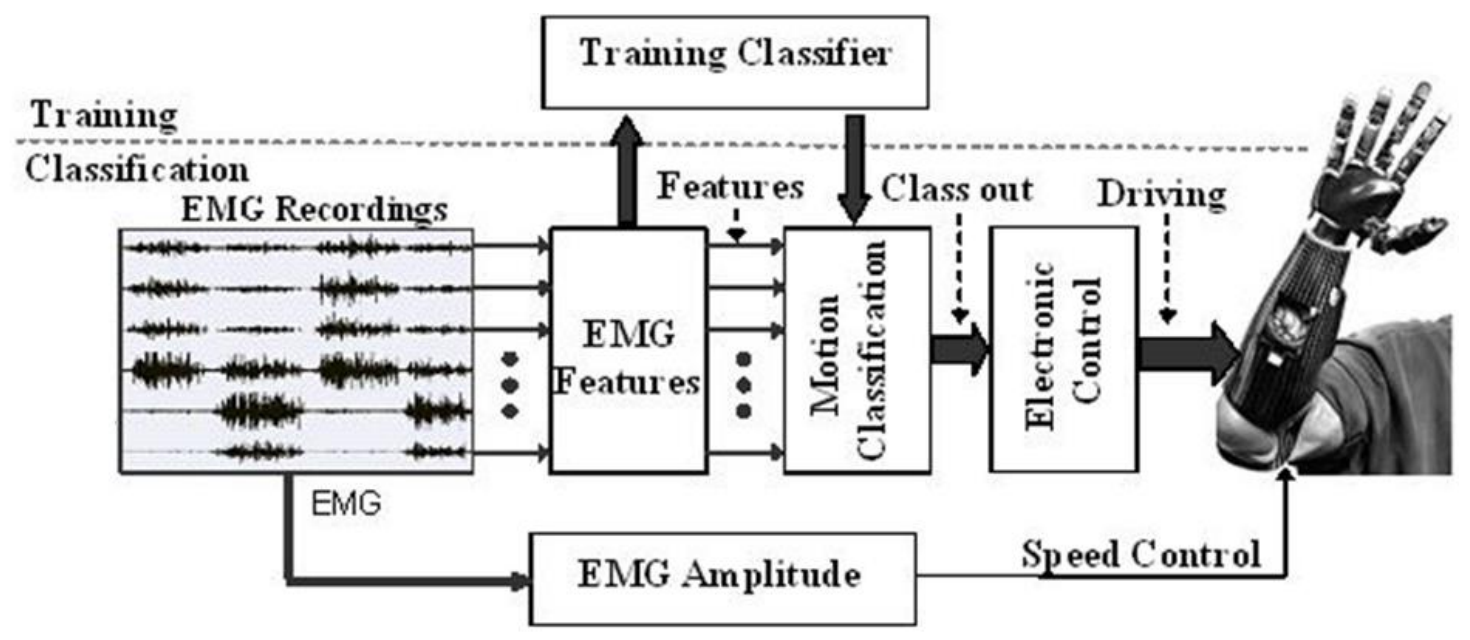

Even though the machine learning algorithms have been in development for long time, their commercial implementation has been delayed until late 2014 with the introduction of the COAPT system [33]. The reasons behind this peculiarity will be discussed in detail in chapter 1.3.

\subsubsection{Feedback interfaces}

None of the myoelectric prostheses currently used in clinical practice have purposely designed closed-loop controllers. Therefore, all current SoA feedback interfaces are still in the laboratory development phase, with the exception of a rather simple one integrated in the Vincent Evolution 2 Prosthesis (Vincent Systems GmbH [34]). Even without its commercial counterpart, prosthetic feedback is a very relevant research topic as summarized in the expert-review article by Antfolk et al. [35]. There are many pathways available to close the loop between the user and the prosthesis (Figure 1.8); but the prevalent one is the non-invasive (i.e., cutaneous). Methods for delivering 
cutaneous feedback fall into two groups: modality-matched or sensory substitution.

Figure 1.8: Three possible feedback information pathways in the context of myoelectric prostheses. Different colors correspond to different pathways $(A, B, C)$. Pathway $A$ is related to sensory information that is directly fed back to the CNS (e.g., visual and auditory feedback); Pathway B to the information that is conveyed to functional sensory motor systems invasively or noninvasively; Pathway $C$ is related to the intrinsic feedback. Image adapted from [35].

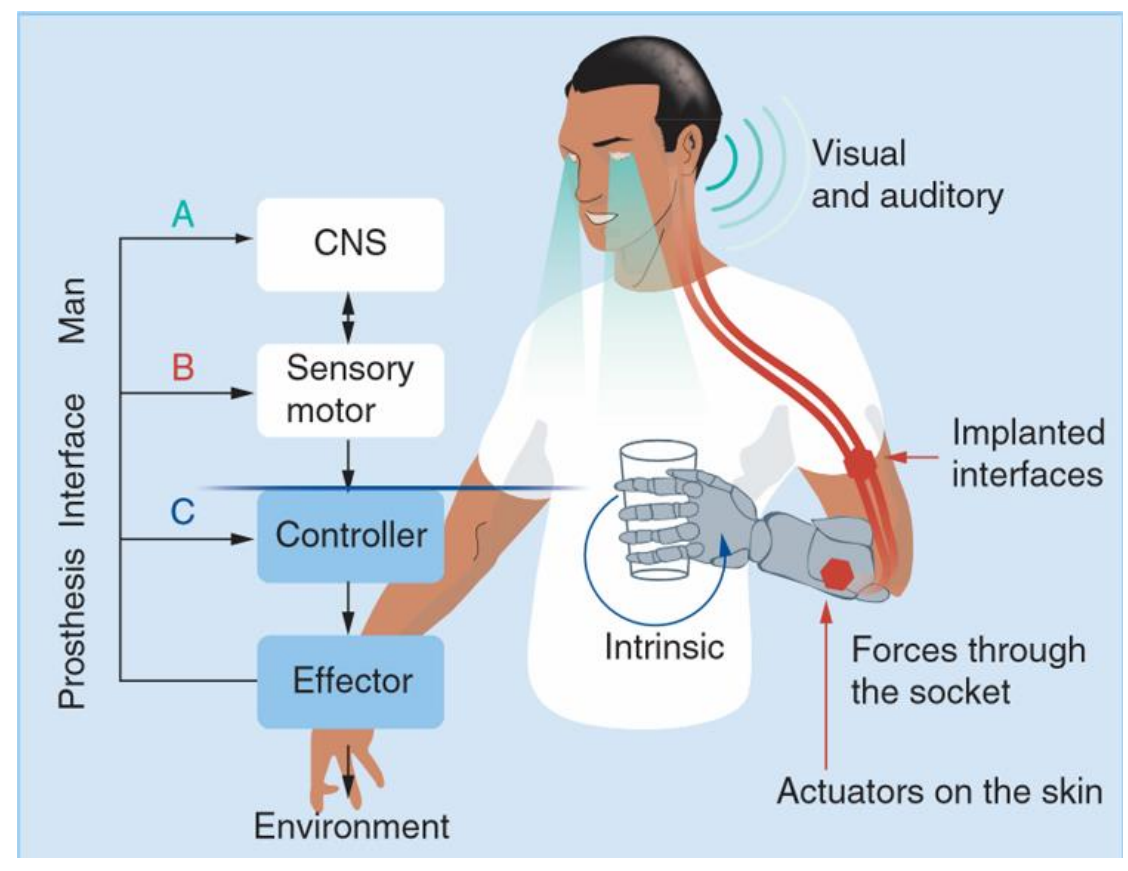

\subsubsection{Modality matched SoA}

Feedback is modality-matched when the output stimulus is felt in the same modality as the sensory input (e.g., temperature sensation is not substituted but rather directly transmitted by warming/cooling the skin surface). The development of non-invasive modality-matched feedback comes along with an array of unique challenges. In theory, it is possible to regain modality-matched touch sensations (contact, normal and shear force/pressure, vibration, texture, temperature) using noninvasive electromechanical devices coupled with thermoelectric ones (Figure 1.9). In their work, Kim et al. [36] and Armiger et al. [37] presented a miniature SoA haptic device capable to transmit touch, pressure, vibration, shear force, and temperature to the skin of the user.

Perhaps the most challenging sensory input to replace is a proprioception. Here the joint angle (e.g., of the elbow) needs to be transferred to another unaffected joint, in order to match the modality. One possible solution to this problem was proposed by Goodwin et al. [38] and later exploited by Roll et al. [39]. They demonstrated that when vibration of around $80 \mathrm{~Hz}$ frequency and sufficient intensity is applied over the tendons at the wrist, the subjects perceive it as a joint motion. This phenomenon could be utilized to 
transmit the prosthesis position in a modality matched way. Therefore, contrary to their intuitiveness, the modality-matched interfaces remain secondary to sensory substitution; crucial obstacles for their successful implementation remain their size, interface, and power consumption.

Figure 1.9: a) Mechanical-and b) thermal-tactor used for modality matched feedback. Images adapted from [37].

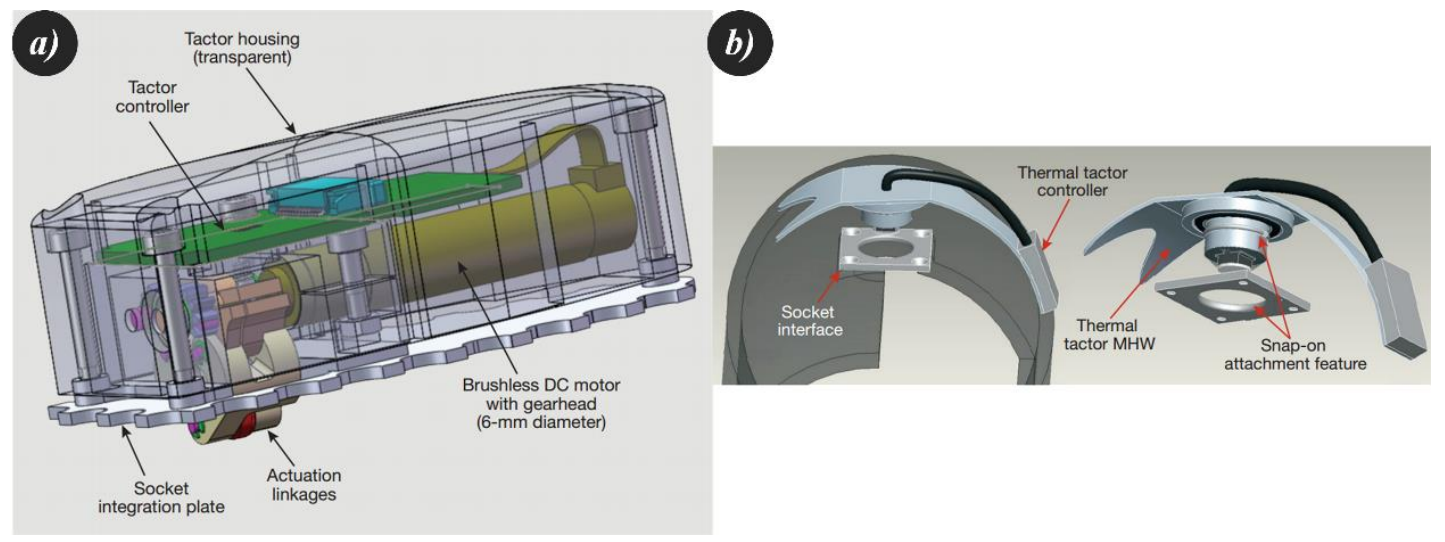

\subsubsection{Sensory substitution SoA}

Sensory substitution is a method to provide sensory information to the body either via a different sensory channel or by maintaining the same channel but by changing the modality. Typical examples of this include the substitution of vision with touch (e.g., Braille alphabet for visually impaired people) or of pressure with vibration. Its main drawback is the danger that the mapping between the physical variable and its representation could be unintuitive to the user. Even though it is not ideal, this technique has the virtue of a relatively straightforward implementation, which is the induction of either mechanical vibration (vibro-tactile) or electric current into the surface of the skin (electro-tactile) (see Figure 1.10).

The vibro-tactile stimulation is elicited on the surface of the skin by mechanical vibrations of the actuator or its contact tip. First such devices, developed specifically for prosthesis application and introduced in the early 50s, were quite bulky and power consuming [40]. But over time, the technology was perfected and they were made much more compact and energy efficient [41], [42]. Vibrotactile feedback activates mainly two types of mechanoreceptors in the skin: Pacinian corpuscles which react best to frequencies between 200 and $300 \mathrm{~Hz}$, and Meissner corpuscles which are best activated by frequencies around $50 \mathrm{~Hz}$ [43]. The sensitivity to amplitude changes is highly dependent on the location. The detection threshold is lowest on the fingertips $(0.07 \mu \mathrm{m}$ at $200 \mathrm{~Hz})$ and highest on the abdomen and the gluteal region (4-14 $\mu \mathrm{m}$ at 
$200 \mathrm{~Hz}$ ) [44]. Until now, vibro-tactile feedback has been used for feeding back a variety of prosthesis states to the user. Some of the more noticeable applications include transmission of prosthesis force, velocity, aperture or elbow position [45], [46], [47], [48].

Figure 1.10: a) The SoA C2-Tactor can be used for vibro-tactile stimulation (Engineering Acoustics, Inc, Florida, USA [42]); b) a disposable surface electrode typically used for electro-tactile stimulation (Spes Medica Genoa, Italy))

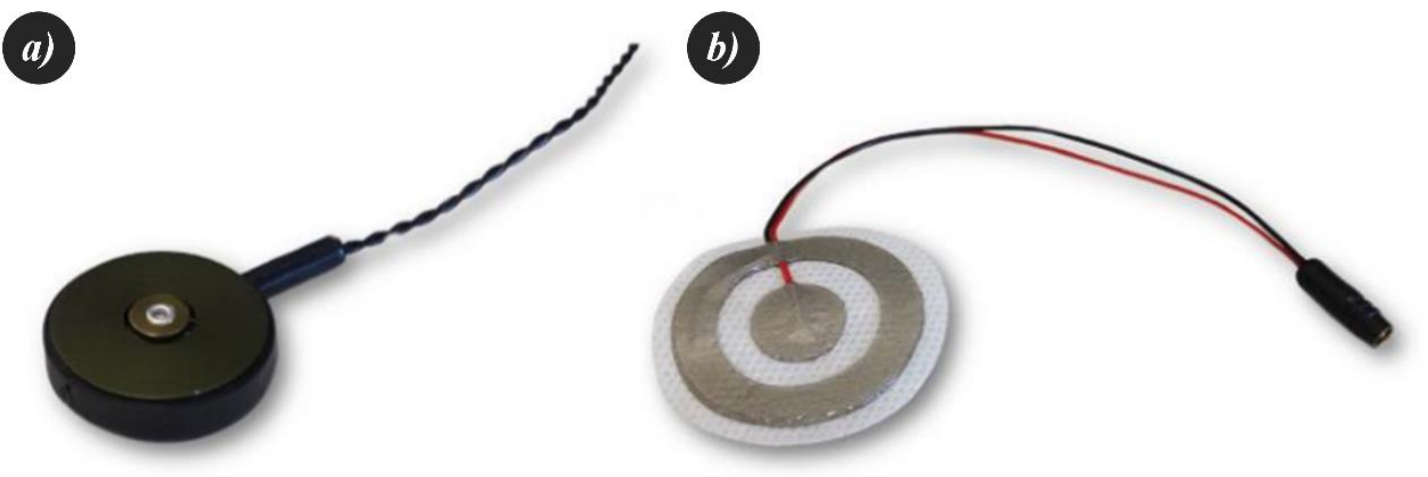

Electro-tactile stimulation induces an electric current originating from a surface electrode (e.g., typically gold, platinum, silver, or stainless steel) that passes through the skin and directly stimulates afferent fibers [49]-[51]. The current polarity and the size of the electrode determine how deep it penetrates the skin surface. This influences the type of sensory afferents activated, since the four types of mechanoreceptors are located at different depths of the dermal tissue [52]. The resulting sensation can be perceived as tingling, itching, buzzing and pinching as well as sharp, needle-like pain. In summary, the parameters of the stimulation (current, frequency, and pulse width) play an important role as do material, type, and size of the electrode, its placement location, and skin impedance. Even though initial research was conducted already in the early 70 s [53], the application of the electro-tactile stimulation interface was delayed until the early 80 s, primarily due to the interference to the EMG signals. Nowadays this pitfall is successfully resolved by using time or frequency division multiplexing [54], [55]. Similar to their vibro counterparts, the electro-tactile devices have been used in a variety of studies as the interface of choice that communicates prosthesis' grip, finger force, or touch [56], [57], [58].

Overall, the two interfaces are functionally very similar and the choice between the one or the other is driven by practical considerations such as power consumption or available space and psychological implications - amputees that suffered from an electrical shock might be negatively predisposed towards electro-tactile stimulation. 


\subsection{Motivation for going beyond the SoA}

"All myoelectric controllers based on pattern classification that have appeared in the literature since the 1990s have provided similar performance (>90\% classification accuracy) ... This conclusion heavily collides with the clinical practice and commercial data: amplitude-based myoelectric control (and not pattern classification) is used in all commercial devices and only a quarter of patients with an upper limb amputation use a myoelectric prosthesis." [59]

"Ironically, after decades of research in upper limb prosthetics, robotics, haptics and applied neuroscience, it is the very simple architecture of the body-powered prosthesis dating back to 1912, that remains the only device coming close to providing physiologically correct and acceptable sensory feedback to the user. " [35]

In a meta-study from 2007 [6] the authors performed a comparative survey of upper limb prosthetics use, acceptance, and abandonment for the past 25 years. The overall results suggested that the rejection rates of the myoelectric hand prostheses have remained exceptionally high at an average of $23 \%$ over time, despite undeniable technological advances. Another study [60] from 1995 confirms these data. Going deeper into the nature of use of prosthetic devices, one interesting subgroup emerges: amputees fitted with an active body-powered or myoelectric prosthesis that use their prosthesis in a passive way. The prevalence of this group is reported to be at $27 \%$ of all prosthetic wearers [6]. Taking into account the aforementioned complexity and cost of myoelectric prosthetic devices, these numbers are even more astonishing and suggest that there are serious pitfalls related to their usage:

- Prosthesis use is very challenging for ADLs such as hygiene, eating, grooming, and manual work [6], [61].

- The overall weight is perceived as high, whereas cosmetics and comfort are often perceived as low [6], [61].

- Low embodiment and lack of feedback: The amputees use their prosthesis more like a tool and less like a part of their own body. Except for one [34], there is no commercial system that offers even the most primitive feedback to the user [29].

If to the list of the aforementioned rejection-factors, we add that most of the amputations are unilateral and $90 \%$ of ADLs can be performed single-handed [62], the reported high rejection rates come as no surprise. It can therefore be concluded that 
current myoelectric prostheses are often perceived as too complicated and functionally irrelevant to be utilized. In comparison to the body-powered and cosmetic prostheses, the ratio between the gained function/improvement on one side and the invested price/effort on the other is not sufficiently attractive.

During the last two decades myoelectric prostheses have improved both in appearance, durability and functionality offering a dexterous and visually very appealing replacement for lost limbs [12], [63], [64], [65]. But if they have become more dexterous and better in appearance, how come that their acceptance rate has not greatly increased? The answer is that it is the man-machine-interface and not the prosthesis design/dexterity that has become a bottleneck for its successful application and overall user satisfaction.

What has not changed over the course of the past 50 years is how prostheses are controlled. The commercial myoelectric prostheses remain interfaced, as before, with proportional two-site electrode systems that usually implement mode switching for multi-DoF control. This means that by increasing the dexterity of the prosthesis the users find themselves in an inconvenient situation: they need to perform more and more time-consuming, and unintuitive mode switching [66]. If this interface is so unintuitive for multi-DoF control, why has it remained the most popular one to this date? In a recent expert article about the future of myoelectric prostheses [59] the authors reflect on the current academic and commercial MMIs and conclude that there is an increasing gap between the two: the impressive advancements from academia fail to be translated into the clinical setting. The often reported above $90 \%$ accuracy in almost all SoA pattern recognition-algorithms should be a clear indication that the long-awaited intuitive control paradigm has arrived; yet, these sophisticated algorithms remain in the lab. One possible reason for this is analyzed in [67] where a study, addressing the performance and usability of 36 myoelectric controllers, demonstrated that the relation between classification accuracy and controller usability/performance is absent or very weak at best. Another hypothesized culprit of this disappointing result is the almost unanimous agreement among the research community to exploit the EMG as the only "true" information source for prosthesis control.

It can be concluded that the SoA prosthesis control, independent of its origin (academia or commercial), follows a common master-slave state-flow. In this configuration, the user generates the commands (master) using residual muscles, the commands are acquired and decoded by the prosthesis controller (slave), and translated into actions 
(i.e., prosthesis movement). This paradigm is confronted with two paradoxes:

- First, it assumes that the user intentions can be inferred by evaluating only the muscle activity while it is well known that, in natural settings, decisions are made based on multisensory information. In the context of movement control, the sensors used are vision, muscle spindles, Golgi tendon organs, joint receptors, skin receptors, and the overall neural network at several levels of the central nervous system. The muscles are just the end-effectors that, to some extent, reflect our intention, but not the overall complexity of the decisionmaking process.

- Second, it assumes that there will always be a sufficient number of muscles to infer the user intention from, which is contradicting basic physiology; namely, the higher the amputation level the less arm muscles are available, but more prosthesis functions need to be controlled (Figure 1.11).

Figure 1.11: Amputation paradox: the higher the amputation level, the less muscles are available to control the increasing number of prosthesis functions.

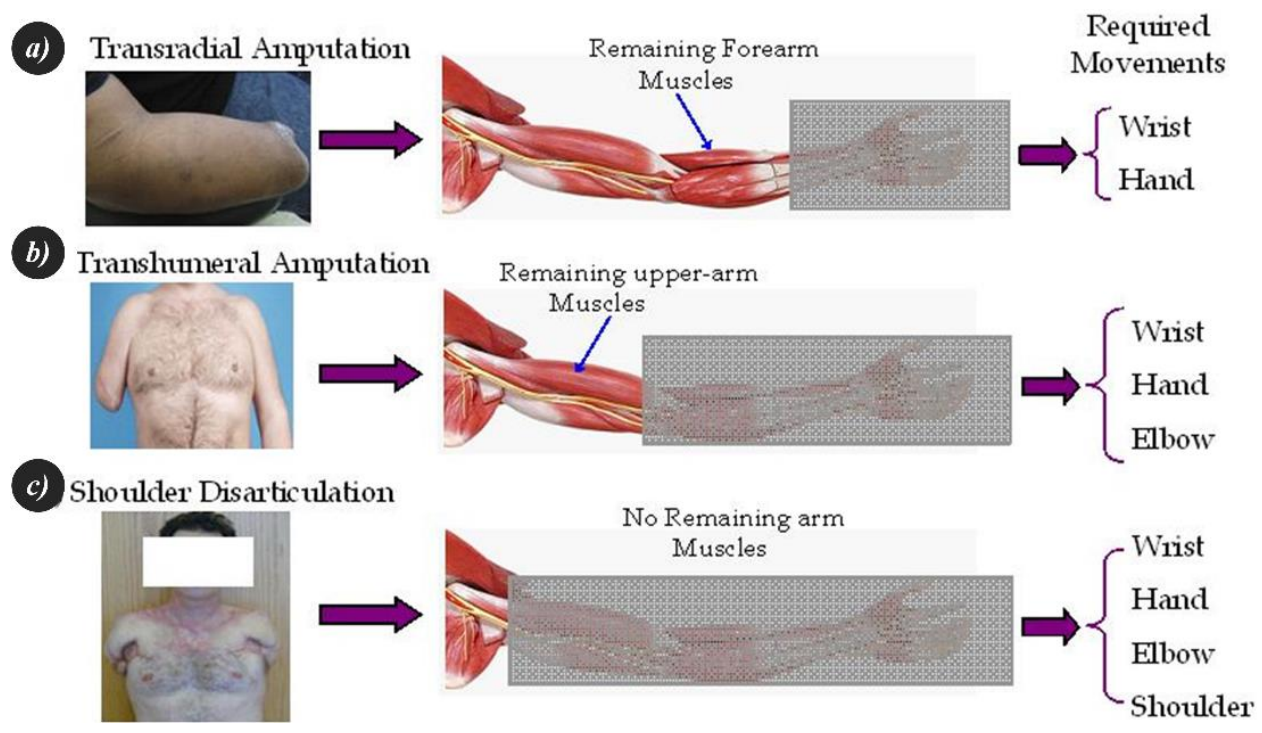

A similar spiral of problems occurs when analyzing the SoA prosthesis feedback. Since natural grasping heavily relies on tactile feedback, it is a widely accepted that the user should benefit from closed-loop control [35], [68]. However, there are many fragmented, often contradictive, attempts to confirm this hypothesis; and since the commercial implementation is lacking it could be said that their overall result is likewise disappointing.

In this thesis, it is hypothesized that there are two main contributors to this state. One is the aforementioned diversity of the feedback interfaces, modalities, and variables, 
which consequently increases the overhead during implementation and experimental validation of closed-loop systems (CLS), and thus renders the acquired results difficult to compare and evaluate (see Figure 1.12). The other one lies in the somewhat overstressed importance of restoring tactile sensation which is most commonly related to feeding back the grip force [35]. However, as demonstrated in [45], feeding back the force information has a limited utility, since closed-loop control can be exploited only during a small fraction of the normal grasping task which is performed relatively fast [1]. Moreover, it is also well known that humans make use of feedforward models of motor commands and prosthetics dynamics in order to predict the outcome [69], [70]. In this light, the SoA feedback modalities and their application need to be reevaluated if we want to advance the acceptance and functionality of myoelectric hand prostheses.

Figure 1.12: Diversity of choices when designing a typical CLS. Typical CLS needs at least one component from each of the columns $(a), b), c), d)$, and e)). The components can be combined almost arbitrary which renders a virtually infinite number of CLS that can be designed.

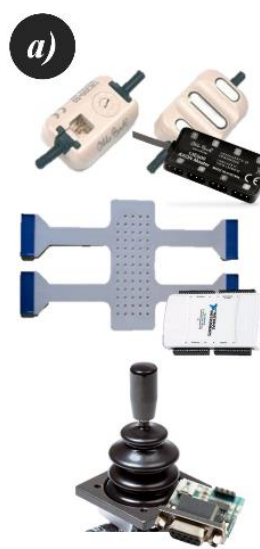

Input interface

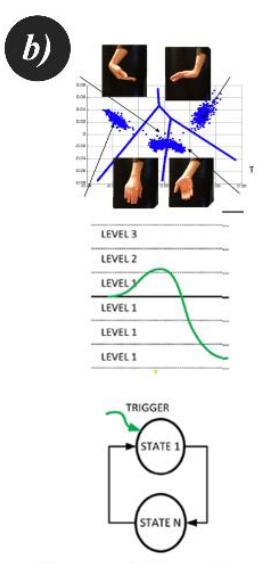

Control Interface c)
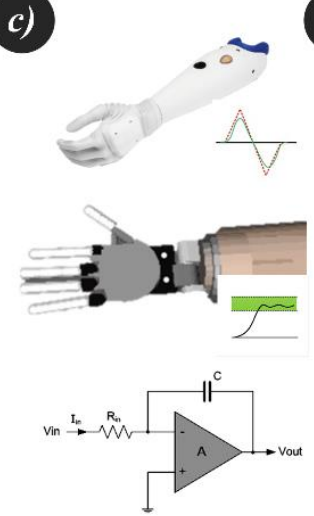

Control system d)
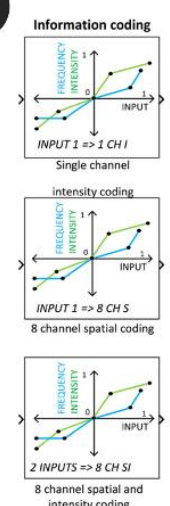

Feedback mapping Feedback interface

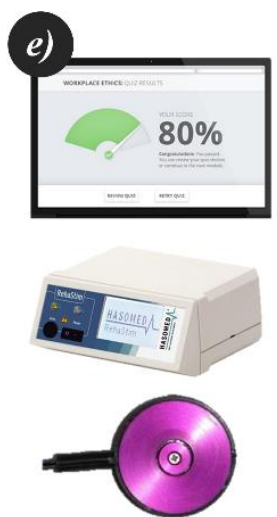




\subsection{Aims of the thesis}

In this thesis a variety of methods and system prototypes, designed to tackle the aforementioned pitfalls of SoA feedforward and feedback interfaces, are presented. More specifically, this thesis aims to address and overcome: 1) the inherently small bandwidth and robustness of the sEMG control channel; 2) the arising difficulties when designing and cross comparing various closed-loop control systems; and 3) the feedback role and its application in the context of prosthesis control.

To this end, the following chapters will briefly summarize the work presented in four peer-reviewed journal publications attached in the appendix:

- "Stereovision and augmented reality for closed-loop control of grasping in hand prostheses" (Appendix 1, [71])

- "Sensor fusion and computer vision for context-aware control of a multi degreeof-freedom prosthesis" (Appendix 2, [72])

- "Sensory feedback in prosthetics: a standardized test bench for closed-loop control" (Appendix 3, [73])

- "EMG Biofeedback for online predictive control of grasping force in a myoelectric prosthesis" (Appendix 4, [74]) 


\section{MATERIALS AND METHODS}

In this section, the reader is introduced to the methods and system prototypes together with corresponding experimental setups that were used to evaluate them.

\subsection{Addressing Prosthesis Control: Sensor-fusion Concept}

It is apparent that apart from assessing muscle activity, other methods and signal sources should also be considered when decoding the users' intentions in prosthesis control. Drawing inspiration from robotic research and applications [75], [76], a new approach has been proposed: multimodal sensor-fusion. Namely, if equipped with an array of multimodal sensors, the prosthesis controller could emulate the high-level processes traditionally considered the responsibility of the user. By fusing information from different sources, the controller is also able to detect and analyze the current context, plan the grasping action, and simultaneously and proportionally control multiple DoFs available in the prosthesis.

In one exemplary application, a novel controller was equipped with artificial vision and proprioception to perceive the state of the user, the prosthesis, and the environment. Based on this information, the controller could make autonomous decisions and automatically configure the prosthesis parameters, simultaneously and proportionally adjusting multiple DoFs according to the task demands and reactively to the user's intentions. This method could be implemented by using sensor-fusion that exploits a unique and comprehensive combination of sensing units comprising myoelectric recording, computer vision, inertial measurement, and embedded prosthesis sensors (position and force) in order to endow the controller of a multi-DoF prosthesis with the 
abilities that are usually reserved for advanced robotic systems. This would allow for continuous and simultaneous perception of the user (proprioception), the environment (exteroception) and their interaction, leading to simultaneous and proportional control of multiple DoFs through context-dependent behavior (e.g., reactive response). Importantly, this approach is not designed to implement control that is independent of the user's actions, but rather to be seamlessly integrated with them.

Figure 2.1: Comparison between a) SoA myoelectric control system and b) the novel context- and user-aware prosthesis controller (CASP). With CASP the prosthesis is controlled semi-autonomously by fusing signals acquired from the user and from an array of additional external sensors; the CASP controller thus reduces the overall user throughput by taking care of the increased prosthesis complexity (note how the line thickens change between the user, the controller and the prosthesis from a) to b)).

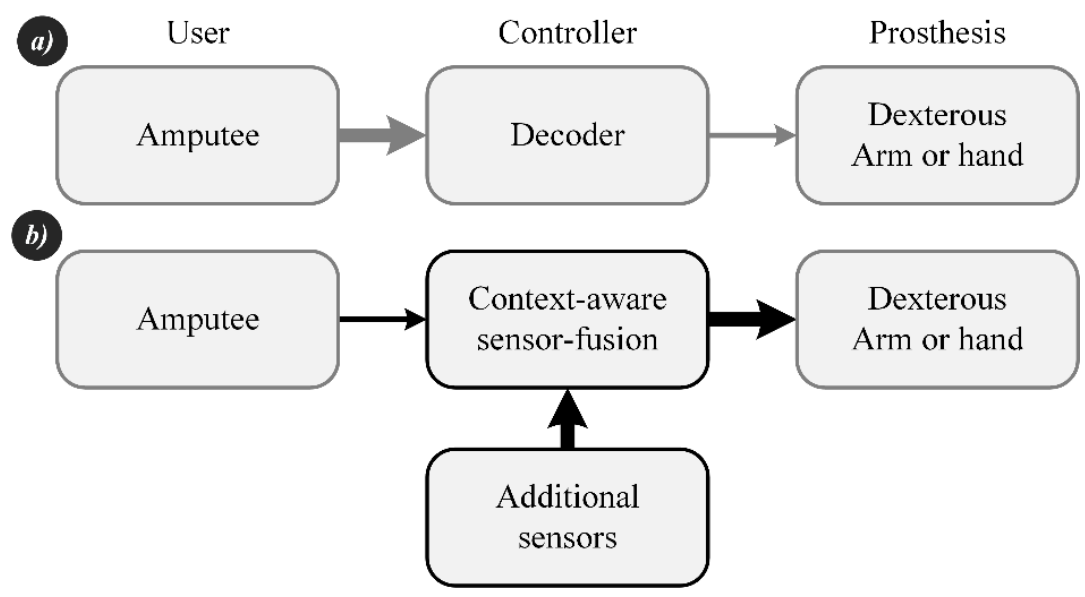

Therefore, in contrast to the previously described master-slave configuration (Figure 2.1a) and its simple "decoder", a context- and user-aware prosthesis (CASP) controller (Appendix 1-2, [71], [72], [77]) was introduced (Figure 2.1b). The CASP is characterized by:

- A semi-autonomous reactive, context- and user-aware prosthesis control that is seamlessly integrated with the user's intentions.

- System scalability: the system can be easily scaled to control an arbitrary number of DoFs (e.g., individual-finger articulated hand or full transhumeral prosthesis), while the user control interface remains virtually unchanged.

The CASP system was developed iteratively, which resulted in two prototypes, described and evaluated in two separate studies (Appendix 1-2, [71], [72]), hereafter Study A and Study B respectively. Since they can be both related to the same conceptual idea, in the following only the second (Appendix 2, [72]), more advanced one, will be described in more detail; key differences between the two are summarized 
in Table 2.1. Hereafter, unless explicitly stated, when referring to CASP, the newest system iteration will be referred. Additionally, some peculiarities of the first system (i.e., the usage of the augmented reality [AR] to close the loop) will be shortly discussed in section 4.3.

Table 2.1: The key-differences between the previous and current system iteration are summed up in the table below and are divided into two sections: features and technical specifications.

\begin{tabular}{|c|c|c|}
\hline & CASP early prototype [71] & CASP [72] \\
\hline \multicolumn{3}{|c|}{ Technical specifications } \\
\hline $\begin{array}{l}\text { Execution speed } \\
\text { and overall } \\
\text { responsiveness }\end{array}$ & $\begin{array}{l}\text { Lower; Slow single- threaded } \\
\text { object modeling }\end{array}$ & $\begin{array}{l}\text { Higher; Usage of the CPU/GPU } \\
\text { multithreading in order to speed up } \\
\text { the object modeling performance }\end{array}$ \\
\hline $\begin{array}{l}\text { Algorithm } \\
\text { complexity }\end{array}$ & $\begin{array}{l}\text { Higher; the depth image needs to } \\
\text { be calculated by matching two } \\
\text { stereo images }\end{array}$ & $\begin{array}{l}\text { Lower; the depth image is obtained } \\
\text { directly from the camera sensor }\end{array}$ \\
\hline $\begin{array}{l}\text { Algorithm } \\
\text { robustness }\end{array}$ & $\begin{array}{l}\text { Lower; specifically, in less } \\
\text { structured/textured environments }\end{array}$ & $\begin{array}{l}\text { Higher; The depth-cameras are the } \\
\text { current SoA }\end{array}$ \\
\hline Employed sensors & $\begin{array}{l}\text { EMG electrodes, stereo-camera, } \\
\text { embedded position encoders }\end{array}$ & $\begin{array}{l}\text { EMG electrodes, depth camera, } \\
\text { inertial sensor, embedded position } \\
\text { and force encoders }\end{array}$ \\
\hline \multicolumn{3}{|c|}{ Features } \\
\hline $\begin{array}{l}\text { Semi-autonomous } \\
\text { control }\end{array}$ & $\begin{array}{l}\text { Yes: grasp type (palmar, lateral, } \\
\text { bidigit, or tridigit) and apareture } \\
\text { of hand prosthesis }\end{array}$ & $\begin{array}{l}\text { Yes: orientation, grasp type, and } \\
\text { aperture of transradial prosthesis }\end{array}$ \\
\hline $\begin{array}{l}\text { Augmented reality } \\
\text { feedback }\end{array}$ & $\begin{array}{l}\text { Yes: utilized during the } \\
\text { experiments }\end{array}$ & $\begin{array}{l}\text { Yes: not utilized during the } \\
\text { experiments }\end{array}$ \\
\hline $\begin{array}{l}\text { Intention-detection } \\
\& \text { user awareness }\end{array}$ & No & Yes \\
\hline $\begin{array}{l}\text { Contextual object } \\
\text { analysis }\end{array}$ & No & Yes \\
\hline
\end{tabular}

\subsubsection{Hardware implementation: CASP}

The CASP prototype utilized the user's voluntary myoelectric commands, computervision as well as the inertial sensing to steer the prosthesis. It comprised the following components (Figure 2.2):

1) The myoelectric interface comprised two 13E200 dry EMG electrodes with integrated amplifiers (Otto Bock Healthcare GmbH, Vienna, AT) placed on the forearm over the wrist and hand flexor and extensor muscles. The electrodes directly provided smoothed signals (linear envelopes). The linear envelopes were sampled at $100 \mathrm{~Hz}$ and transferred to the host PC via a Bluetooth (BT) connection. 
2) A Creative Senz3D (Creative Technology Ltd., SG) camera simultaneously acquired color and depth images (RGB-D) [78] and was mounted on the customdesigned support glasses worn by the subject, thus ensuring that the camera was always directed towards the same scene at which the user was currently looking. The acquired image streams were transmitted to the host PC via a USB port at a $30 \mathrm{~Hz}$ refresh rate and a resolution of $640 \times 480$ pixels for RGB and $320 \times 240$ pixels for depth images, respectively.

3) The Michelangelo Hand (Otto Bock Healthcare GmbH, Vienna, AT) provides simultaneous opening and closing of all fingers with two grasp types (palmar and lateral), as well as wrist pronation and supination (i.e., three DoFs in total) [12]. The hand was instrumented with three position encoders (thumb, fingers, and wrist) and a single force transducer positioned at the base of the thumb, measuring the hand aperture, grasping force, and hand orientation relative to the socket. The prosthesis was connected to the host PC via a BT interface implementing a bidirectional communication protocol running at $100 \mathrm{~Hz}$. This communication channel was used to receive the sensor data from the prosthesis and to send the control commands to the prosthesis.

4) The inertia measurement unit (IMU) (Xsens-MTx Technologies B.V., Enschede, NL) was externally attached to the prosthesis. The IMU measured the absolute orientation of the prosthetic hand with respect to the laboratory coordinate system, i.e., yaw, roll, and pitch angles. The IMU was connected to the batterypowered acquisition and wireless transmission unit sending data to the host PC at a sampling rate of $20 \mathrm{~Hz}$.

5) The data from the prosthesis, IMU, and camera were received by the processingunit (i.e., standard PC with 8GB RAM and i7@2.9 GHz CPU), where data processing, sensor fusion, and control algorithms were performed. The host PC also provided a graphical user interface (GUI) for high-level control (e.g., starting and stopping) and system monitoring and setup. 
Figure 2.2: a) The relevant components of the CASP prototype are shown. b) The CASP system prototype utilized by an amputee to grasp a book. Image adapted from [72].

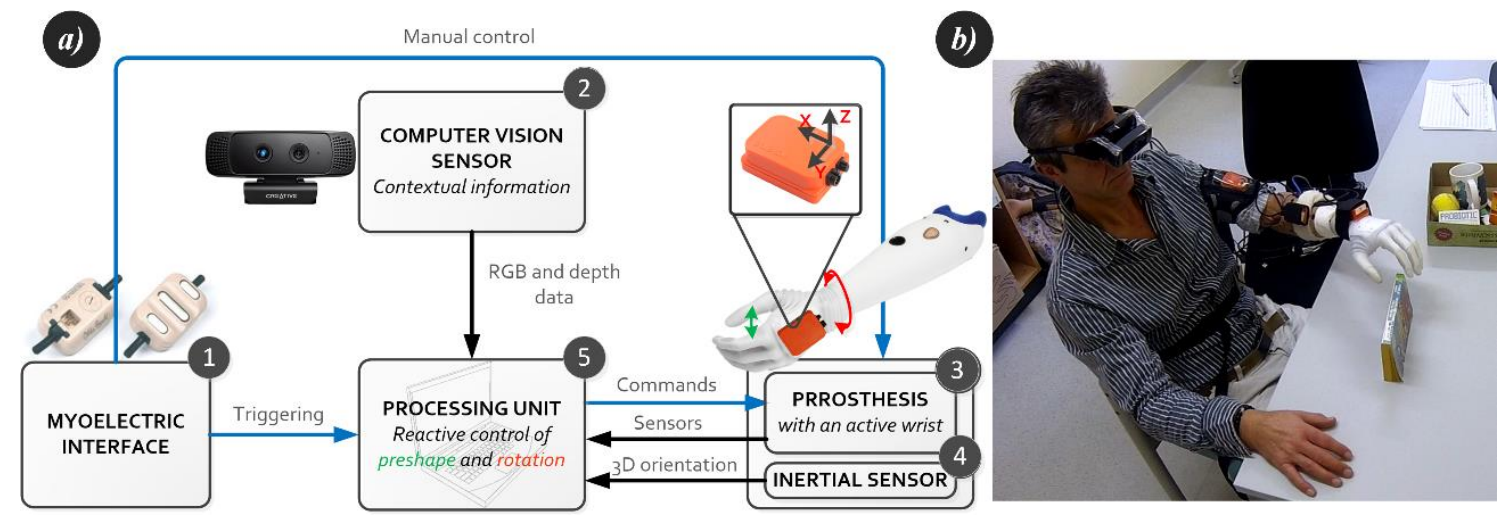

\subsubsection{Software implementation: CASP}

The algorithm driving the CASP system prototype was implemented in MATLAB 2013a (MathWorks, Natick, US-MA) as a library of individual modules using object oriented programming. Due to the specific performance requirements (e.g., intensive real-time calculations and data processing) additional application-specific computational optimizations were implemented using custom compiled C-code and CPU/GPU multithreading [79].

The system operated as a finite-state machine that is triggered by myoelectric commands or external events (e.g., object grasped/released). That is, when the user generated a myoelectric trigger command, the processing unit fused the data acquired from the sensors (i.e., prosthesis aperture, grasp type, orientation, and depth image) in order to perceive the environment (i.e., the graspable objects in it) and performed automatic and real-time updates of the prosthesis parameters. Based on the current state of the prosthesis and the estimated properties of the target object (shape, size, orientation), the prosthesis posture (i.e., grasp type, size, and wrist angle) was configured so that the hand is prepared for grasping the target object (reactive control). Additionally, once the prosthesis posture has been automatically adjusted, the user would regain a full manual control of the prosthesis through the myoelectric interface, thus being able to correct or fine-tune the autonomous decisions (semi-autonomous control).

As already stated, the core feature of the CASP algorithm (Figure 2.3) is the fusion of sensor data from several sources, including IMUs, depth camera, embedded position, and force sensors. This comprehensive sensor fusion (red dashed-line box) allows the 
algorithm to implement automatic, simultaneous, and reactive position control of the multi-DoF prosthesis. The inputs for the processing are: depth image (acquired via the infrared time-of-flight camera), intrinsic prosthesis properties (e.g. handedness, number of available DoF's), the IMU's orientation (attached externally to the prosthesis) and the data acquired from the sensors embedded into the prosthesis (force sensors and position encoders). The outputs are the control signals that automatically configure the prosthesis into the predefined posture by setting the grasp type, aperture size, and wrist rotation.

Figure 2.3: Conceptual scheme of the algorithm driving the CASP system. The central feature of the system is the sensor fusion, which allows for context-dependent reactive prosthesis control. List of abbreviations: Hand preshape and orientation (HPO), object of interest (OI), current prosthesis rotation (current_Rot), rotation of the selected hand posture (HPO(i)_Rot).

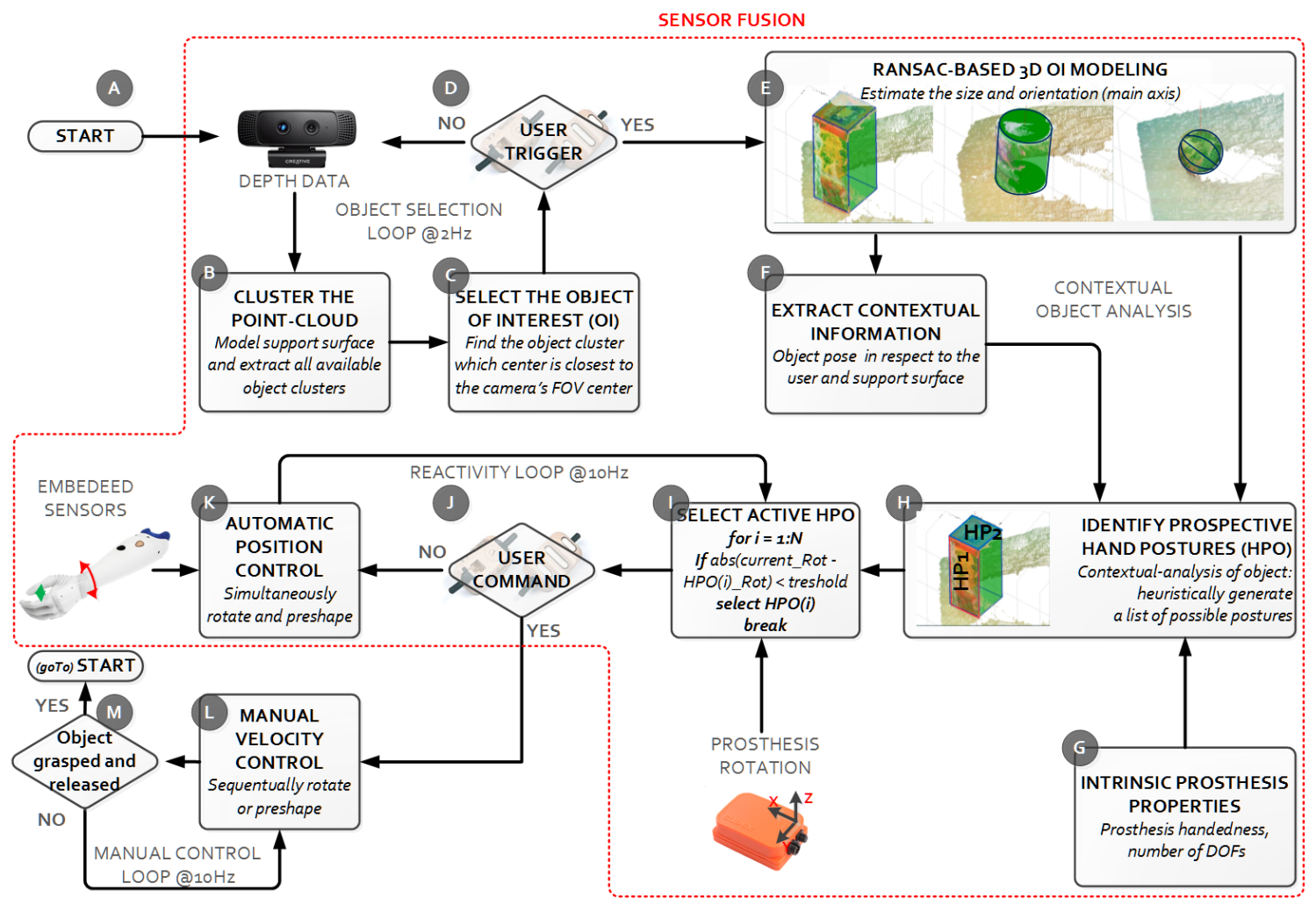

\subsubsection{Object selection loop (Figure 2.3, blocks A-D)}

The object selection loop analyzes the acquired depth images in order to extract the object of interest (OI) cluster from the 3D point-cloud. It operates at $2 \mathrm{~Hz}$, until the user generates a myoelectric command for hand opening. The aforementioned object segmentation is performed as a three-step process in which:

1) The support surface (step B) is determined by identifying the largest plane in the point-could through iterative application of the RANSAC [80] algorithm for 
plane detection.

2) All points that are above the support plane are considered to belong to a single cluster containing all present objects. This large cluster is further divided into smaller ones, each assuming to contain a single object. This is accomplished by detecting the discontinuities in the point-cloud through edge analysis applied to the raw depth image (Canny edge detector [81]). Additionally, some clusters are eliminated using post-processing. More specifically, all clusters that do not satisfy the requirements for a valid object defined as a set of thresholds are discarded (e.g., clusters that are too much dispersed, too small, or too far away are eliminated in the process);

3) From the object clusters, extracted in step 2, the one closest to the center of the camera's field-of-view (FoV) is considered the OI (step C).

Therefore, the results of the initial object selection loop are two point-cloud clusters: one belonging to OI and the other belonging to the support surface.

\subsubsection{Contextual object analysis (Figure 2.3, blocks E-H)}

Once the user issues a hand opening command through the myoelectric interface (step D), contextual analysis of the OI is performed:

1) The RANSAC method is applied once more to iteratively fit four different geometrical models (box, cylinder, sphere, and line) through the point-cloud belonging to the $\mathrm{OI}$ (step E). The model with most inliers is selected as the one best describing the shape of the OI, where the inlier is defined as a point located within the volume of the fitted geometrical shape.

2) The main-axis of the geometrical model is determined as the line passing through the object center, parallel to its longest edge (step E). The main-axis is used to determine the object pose with respect to the support surface and the user (step F).

3) The information obtained from steps $E$ and $F$ is then fused with the intrinsic prosthesis properties (step G) in order to generate the repository of viable hand preshapes and orientations (HPOs, step H). To this aim, the algorithm first identifies all object surfaces that qualify to be grasped. For example: all object surfaces that are facing away from the user/prosthesis are eliminated from further analysis. Then, a cognitive like processing algorithm implemented as a set of IF-THEN rules similar to the ones described in [82], [71], [83] is applied 
iteratively over each qualified object surface in order to generate an appropriate HPO. Therefore, each HPO is defined as a triplet including hand orientation, grasp type, and aperture that depends not only on the object but also on the intrinsic prosthesis properties (e.g., for a prosthesis that has individually controllable fingers the HPO would automatically generate more dexterous preshapes as demonstrated in [71]; Similarly, the object surfaces that qualify to be grasped depend, as well, on the prosthesis handedness). For example: a wide cylindrical object of $\mathrm{X} \mathrm{cm}$ in radius and oriented vertically would be grasped using palmar grasp, and the prosthesis would be oriented so that the palm is either vertical $\left(90^{\circ}\right)$ or horizontal $\left(0^{\circ}\right)$ when grasping from the side or above, respectively. In this process, the hand aperture size would be set to be somewhat larger than the estimated object width. The two HPOs would be therefore determined as: $\left(0^{\circ}\right.$ rotation, palmar preshape, $\mathrm{X} \mathrm{cm}$ aperture $)$ and $\left(90^{\circ}\right.$ rotation, palmar preshape, $\mathrm{X} \mathrm{cm}$ aperture).

Therefore, the outcome of this processing stage is a repository of HPOs that accommodate grasping of the target object from all the viable sides.

\subsubsection{Automatic posture control loop (Figure 2.3, blocks I-K)}

This loop operates at $10 \mathrm{~Hz}$ and fuses the repository of HPOs with the current prosthesis orientation (obtained via IMU) in order to automatically drive the prosthesis into the optimal configuration. The currently measured prosthesis orientation is compared to all HPOs from the repository, and the HPO with the closest orientation is selected as the one to be activated (step I). This is performed at least once, immediately after the contextual object analysis has been finished, in order to configure the initial HPO. Then, due to the continuous activity of this loop, the controller detects if the user tries to orient the prosthesis in order to grasp the object from a different side and activates the corresponding HPO from the repository (reactive behavior). The selected hand posture is then reached, in a closed-loop, using embedded position encoders (step K). It should be noted that the resulting amount of wrist rotation is calculated by subtracting the current socket rotation (obtained via IMU) from the activated HPO rotation (obtained from the repository of HPOs). This amount is then added to the current wrist to socket rotation (obtained via embedded position encoders) and sent as the new rotation command to the prosthesis controller (step K). 


\subsubsection{Manual control loop (Figure 2.3, blocks L-M)}

This loop operates at $10 \mathrm{~Hz}$ and implements a two-site proportional myoelectric control interface with mode switching, as described in Introduction section 1.2.1.1. The loop is entered automatically once the user issues any kind of myoelectric command during the automatic control loop operation. This allows him to voluntarily grasp and manipulate the object of interest or simply correct the system decisions. Once the object has been grasped and released the manual control loop finishes and the state-machine resets automatically to state A.

\subsubsection{Experimental setup and evaluation: CASP}

As already mentioned, the CASP system was developed iteratively, which resulted in two system prototypes. Each prototype was evaluated and published in a separate study [71], [72] (i.e., Study A and B, respectively). Even though there were differences in evaluation, the experimental setup remained virtually unchanged between the two studies. The subjects were seated in front of a table while wearing either of the two system prototype versions, as depicted in Figure 2.4. Their task was to grasp and transport, one by one, objects common to ADLs in accordance with the following instructions:

1) Starting from rest position: turn the head towards the object.

2) Trigger the system by issuing the appropriate myoelectric command and wait until the prosthesis automatically responds (i.e., until it adjusts its posture).

3) Transport the prosthesis to the object and generate appropriate grasp force in order to lift it up.

4) Transport and release the object on the pre-designated place on the table surface.

5) Return to the rest position.

While the experimental setup and the overall task remained unchanged, the experimental scenarios and accompanying evaluation paradigms were somewhat different for Studies A and B. Since it was the first study that introduced a genuinely novel concept, study A was focused on evaluating the overall robustness and feasibility of the semi-autonomous control. On the other hand, in study B, the emphasis was put on performance evaluation in the context of the commercial SoA. The key experimental remarks for both studies are summarized in Table 2.2 and additionally explained in the text below. 
Figure 2.4: Experimental setup/task used for evaluating both iterations of the CASP system. The EMG electrode placement is denoted with "e". The dashed circle represents where the object should be released. Experimental task (1-5) is denoted in blue. (*) The IMU (hexagon) was not implemented in the first system iteration.

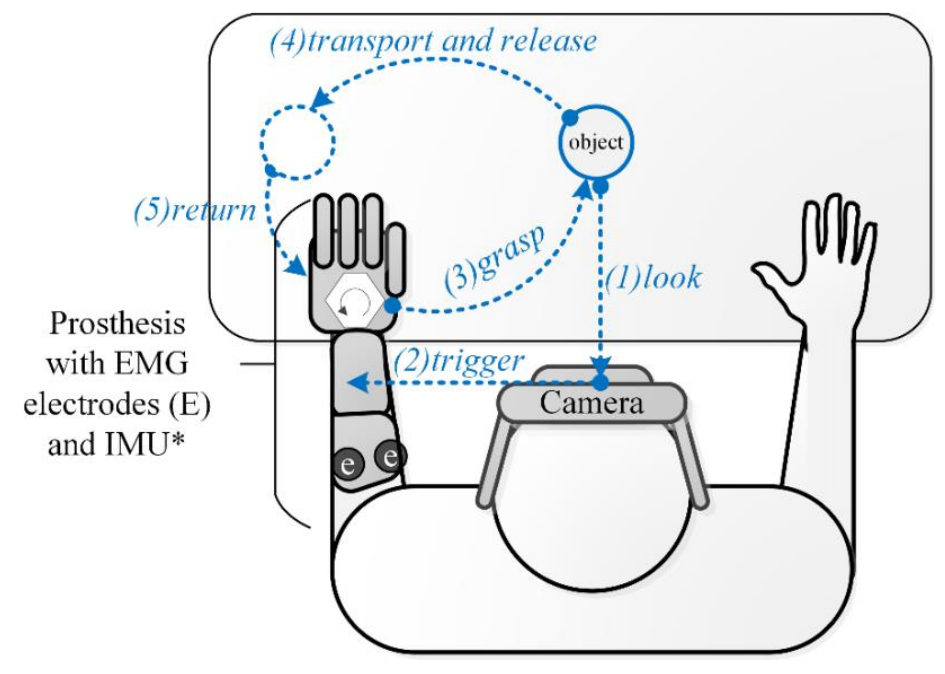

Table 2.2: Summary of the experimental evaluations performed in Studies A and B. (*) For more information, consult Table 2.1. (**) AR feedback is discussed separately in Appendix 1, [71](***) The outcome measures have a different abbreviation in original study.

\begin{tabular}{|c|c|c|}
\hline & Study A & Study B \\
\hline $\begin{array}{l}\text { Experimental setup } \\
\& \text { overall task }\end{array}$ & $\begin{array}{l}\text { Subject sits in front of the table and } \\
\text { grasps different objects }\end{array}$ & $\begin{array}{l}\text { Subject sits in front of the table and } \\
\text { grasps different objects }\end{array}$ \\
\hline System* & $\begin{array}{l}\text { CASP early prototype; Controlling } \\
\text { multi-articulated hand prosthesis ( } 4 \\
\text { grasp types: lateral, palmar, bidigit, } \\
\text { trididgit) }\end{array}$ & $\begin{array}{l}\text { CASP; controlling full transradial } \\
\text { prosthesis (two grasp types: palmar or } \\
\text { lateral and an active wrist) }\end{array}$ \\
\hline Subjects & 13 able-bodied ( $29 \pm 4$ yrs.) & $\begin{array}{l}10 \text { able-bodied }(29 \pm 4 \text { yrs.) and one } \\
\text { amputee ( } 55 \text { yrs.) }\end{array}$ \\
\hline Test scenarios & $\begin{array}{l}\text { Single session: } \\
\text { 1. Full autonomous control (AUTO) } \\
\text { 2. Semi-autonomous control } \\
\text { (SEMI) } \\
\text { 3. Utilization of the AR feedback** }\end{array}$ & $\begin{array}{ll}\text { Two sessions (training and evaluation): } \\
\text { 1. } \\
\text { 1-DoF manual SoA myoelectric } \\
\text { control (MAN 1) } \\
\text { 2. } \\
\text { 3-DoF - II- (MAN 2) } \\
\text { 4. } & \text { CASP - II- (MAN3) } \\
\text { CAStrol (CASP) }\end{array}$ \\
\hline $\begin{array}{l}\text { Number of trials per } \\
\text { scenario }\end{array}$ & $\begin{array}{l}20 \text {; the number of trials equaled to the } \\
\text { number of objects }\end{array}$ & $\begin{array}{l}\text { 17; the number of trials was greater } \\
\text { since some objects were reused in } \\
\text { different orientations }\end{array}$ \\
\hline Objects & $\begin{array}{l}20 \text { ADL objects of different } \\
\text { dimensions }\end{array}$ & $\begin{array}{l}10 \text { ADL objects of different } \\
\text { dimensions; objects presented in } \\
\text { different orientations }\end{array}$ \\
\hline $\begin{array}{l}\text { Relevant outcome } \\
\text { measures }\end{array}$ & $\begin{array}{ll}\text { 1. } & \text { Object size estimation error } \\
& \left(\mathrm{SEE}^{* * *}\right) \\
\text { 2. } & \text { Preshape success rate }\left(\mathrm{PSR}^{* * *}\right) \\
\text { 3. } & \text { Task accomplishment rate } \\
& \left(\mathrm{TAR}^{* * *}\right) \\
\text { 4. } & \text { System-induced task failure rate } \\
& \left(\mathrm{SFR}^{* * *}\right)\end{array}$ & $\begin{array}{l}\text { 1. Time to grasp (TTG) } \\
\text { 2. Object orientation estimation error } \\
\left(\text { OEE }^{* * *} \text { ) }\right. \\
\text { 3. Compensation in the shoulder joint } \\
\text { (angles) }\end{array}$ \\
\hline
\end{tabular}




\subsubsection{Study A}

The study was performed on thirteen able-bodied subjects ( $29 \pm 4$ years), which utilized an earlier version of the CASP prototype that did not feature user- and contextawareness, but only semi-autonomous control of the 4-DoF multi-articulated hand prosthesis (four grasp types). The task (i.e., to grasp and transport different objects one by one) and the experimental setup (Figure 2.4) remained the same regardless of the test scenario. The outcome of the study was the comparison of full- and semiautonomous control:

1) Autonomous control (AUTO) only. In this condition, the manual control loop was switched off, thus rendering the subjects unable to correct any decisions or eventual errors made by the CASP system. They had to accept and work with any decision that the system made for them. This condition was used to assess the baseline performance when the control was fully autonomous.

2) Semi-autonomous control (SEMI). In this condition, the CASP operated according to the full control loop (as described in 2.1.2, but without reactive loop and contextual analysis). This is to say that, in addition to the autonomous control, the system implemented SoA manual myoelectric control that was at the users' disposal. The user could thus react on system decisions and correct them if necessary. This condition was used to evaluate the performance of the CASP system in which the user and the system share the control responsibility.

The two test scenarios were performed always in the same order - first AUTO and then SEMI. Each of the test scenarios comprised 20 grasping trials. In each grasping trial, the subjects were presented with a single object and orally instructed how the prosthesis should be optimally preshaped (e.g., palmar preshape for a bottle, lateral for a pen). There was no time limit for performing the trial. During the SEMI-AUTO scenario, the subjects were instructed to trigger the CASP and correct system decisions if they did not match the orally instructed ones. In the AUTO scenario, where the manual control loop was disabled, the instruction was to grasp the object nevertheless. The following outcome measures were relevant:

- Object size estimation error (SEE): Absolute difference between the actual size (D) and estimated object size $\mathrm{d}$ : OSE $=|\mathrm{d}-\mathrm{D}|$. This measure was used to evaluate the precision of the computer-vision algorithm that clustered and modeled the objects.

- Preshape success rate (PSR): The percent of trials in which both the grasp type 
and aperture size were correct and appropriate. In this case, the hand was perfectly preshaped to grasp the target object.

- Task accomplishment rate (TAR): The percent of trials in which the task was successfully completed (the user grasped the object, lifted it off the table, brought it to the final position and released it).

- System-induced task failure rate (SFR): The percent of trials in which the user failed in the task due to the employed autonomous control logic.

The outcome measures were designed to reflect separately, the performance of the autonomous/semi-autonomous control (OSE, PSR) and the overall task accomplishment rate (TAR, SFR). The justification for this is that the two performance outcomes might be uncorrelated in an absolute sense. Namely, even gross system error that results in wrong hand preshape might lead to successful grasping and task competition (and viceversa). In the case of the semiautonomous control (SEMI), the PSR was measured after subject fine-tuned the system (if at all), whereas in the case of the autonomous control (AUTO), it was estimated directly by taking the decisions of the CASP controller.

\subsubsection{Study B}

In this study, experimental tests were performed on 10 able-bodied subjects (26 \pm 3 years, six with prior experience in myoelectric prosthesis control) and one amputee (55 years, 35 years since amputation, active 1-DoF prosthesis user). They used the newest version of the CASP prototype that featured user- and context- awareness, and was capable of semi-autonomous, reactive control of the 3-DoF transradial prosthesis (active wrist, and two grasp types: lateral and palmar). The task (i.e., to grasp and transport different objects - one by one) and the experimental setup (Figure 2.4) remained the same regardless of the test scenario. The outcome of the study is the comparison of the performance between CASP and three, progressively more complex, manual control scenarios MANn $(n=1,2$, and 3 denotes the number of manually controllable DoFs):

1) MAN1: proportional control of the prosthesis velocity of closing and opening and grasping force, hand in palmar grasp, wrist orientation fixed in the neutral position.

2) MAN2: as in MAN1 plus the subjects selected between the palmar and lateral grasps using co-contractions.

3) MAN3: as in MAN2 plus the subjects proportionally controlled the velocity of 
wrist rotation (pronation and supination), co-contractions were used to switch between the DoFs in the following order: palmar grasp, lateral grasp, and wrist rotation.

4) The CASP system operated according to the full control loop as described in 2.1.2.

The experiments were performed in two separate sessions (training and evaluation), spread across two days. Each of the two sessions comprised the four aforementioned test scenarios in a randomized order and each of them comprised 17 grasping trials. In each grasping trial, the subjects were presented with a single object and orally instructed to adjust the prosthesis so that the hand was configured appropriately for grasping the object (e.g., hand oriented vertically, preshaped palmar for grasping a bottle). There was no time limit for performing the trial. The task execution was assessed visually by the experimenter and the trial was repeated if a gross error had been made and prosthesis had not assumed the correct posture (i.e., the subject or the system employed the wrong grasp type or orientation). One important instruction concerning the orientation was that, if not necessary, the subjects should not use compensatory strategies (e.g., they should not compensate the wrist orientation from the shoulder/elbow joint). The following outcome measures were relevant:

1) The time to grasp (TTG) an object using a specific control scheme (MAN or CASP) assessing the efficacy in operating the prosthesis employing a particular control. The TTG was also used to compare the performance between the training and evaluation sessions for the same control scheme to assess if there was an improvement due to the training. The TTG was measured from the start of the trial until the hand contacted the object (force $>$ threshold).

2) The shoulder joint angles computed from the inertial data, recorded during the evaluation session only assessing the arm configuration just before the hand grasped the object $(0.5 \mathrm{~s}$ before contact). They were calculated as the Euler angles of the upper arm with respect to the immobilized trunk.

3) Additionally, during the CASP control scenario the average orientation estimation error (OEE) was calculated as the difference between the true object inclination angle (e.g., vertical equals $90^{\circ}$ ) and the estimated inclination from the CASP algorithm. This measure was used to evaluate the precision of the computer-vision algorithm that clusters and models the objects. 


\subsection{Addressing Prosthesis Feedback}

In the introduction, it has been stated that one of the main problems in developing and testing CLS is the overall fragmentation of control systems, feedback interfaces, modalities, and devices (for illustration of available choices refer to Figure 1.12). The sole variety of the available feedback modalities makes it very difficult to compare and evaluate them in a scientifically relevant and consistent manner. Additionally, each of the feedback modalities or control systems has its own software interface that is specific to the utilized hardware. Since closed-loop control of a prosthesis comprises a rather sophisticated and complex system, consisting of many components that implement both real-time feedforward as well as the feedback MMI, this often leads to a development overhead. Namely, changing just a single component (e.g., exchanging a vibro- with electro-tactile device) in the prototype might render the whole system unusable and require a lot of additional programming in order to adapt to the new software interface and communication protocol.

The problems of poor inter-component compatibility, algorithm reusability, as well as arising difficulties when comparing results obtained from different prototypes can be solved by abstracting and encapsulating the low-level functionalities in pre-designated placeholders that are interoperable, independent in function, and transparent in behavior. In addition, the placeholders should also be smart, self-configurable, and easily extendable in order to minimize the chance of human error. To this end, a closedloop development framework (CLF) was developed (Appendix 3,[73]). The framework is primarily designed for testing real-time human manual control with sensory feedback. Its overall value is best demonstrated in its application. Shortly after its deployment, several relevant experimental setups and systems, all addressing the topic of feedback in prosthetics, were evaluated and published [69], [74], [84].

One particularly interesting method that was developed and evaluated within the CLF is the usage of biofeedback in order to improve prosthesis control [74]. This method challenges the SoA concept of feedback in prosthetics, leading to functional improvements in comparison to it.

In the following chapters, the implementation of the CLF as well as the novel method for closed-loop prosthesis control (Biofeedback) will be described. 


\subsubsection{Closed loop development framework}

The CLF was implemented using a graphical programming language (Simulink) that is part of the Matlab 2013a (MathWorks, Natick, US-MA) software package. Additionally, Simulink 3D Animation, Simulink Coder, Signal Processing, and Real Time Windows Target toolboxes were used (RTWT). The framework is structured as a library of components that seamlessly integrate into the already existing Simulink Library Browser (Figure 2.5):

- The input interface library implements the generation, acquisition, and preprocessing (e.g., baseline removal, normalization, filtering) of the signals from different information sources (e.g., muscle activity, joystick, data file, predefined signal).

- The control method library implements the mapping from the input level to the system level commands. More precisely, it integrates a variety of control interfaces (e.g., proportional or state-machine control) that translate the input to corresponding system activation (e.g., DoF activation).

- The system library encapsulates a low-level interface to the real or simulated system that is controlled in the closed-loop system. The block defines intrinsic system parameters and the mapping between the control signals and the available system DoFs, defining which control input controls which system function (e.g., for prosthesis: Input 1 controls hand opening/closing, Input2 wrist rotation, etc.).

- The experimental task library compares the current versus a desired system state, as defined by the system block and the goal of the experiment, respectively. It operates in two modes: pursuit and compensatory. Based on the operation mode it determines which signal should be fed back to the user (e.g., in pursuit mode the output will be the desired and the current system state; whereas in the compensatory mode the output will be the error).

- The information-coding library translates the normalized feedback information signals into normalized stimulation parameters (i.e., frequency and intensity) for a generic multichannel feedback device. This library is one of the core CLF features since it allows for designing and reusing any custom-made coding scheme (e.g., intensity or spatial coding), independent of the underlying hardware interface.

- The feedback interface library encapsulates a low-level communication with a 
device that is used to deliver the feedback (e.g., visual, tactile, sound). Each block inside of it sends device specific commands to adjust the stimulation parameters to the normalized frequency and intensity values supplied by the information-coding block.

- The signal-processing library implements the most common signal processing features such as signal filtering, normalization, artefact removal, etc.

- The model-configuration library integrates components that are designed to govern the overall model execution (e.g., simulation duration, sampling times, etc.) and data logging of simulation signals and model parameters.

Figure 2.5: a) The structure of the closed-loop development framework (CLF). The implemented components are divided into eight different libraries organized into separate folders. Each component follows the same structural organization consisting of a component-model (.slx) and dependencies folders. Custom-designed CLSs are saved in a separate folder tree (test bench models). b) CLF integrated in Simulink Library Browser. In order to use it, the user needs to navigate to the desired component and simply drag and drop it into his model.

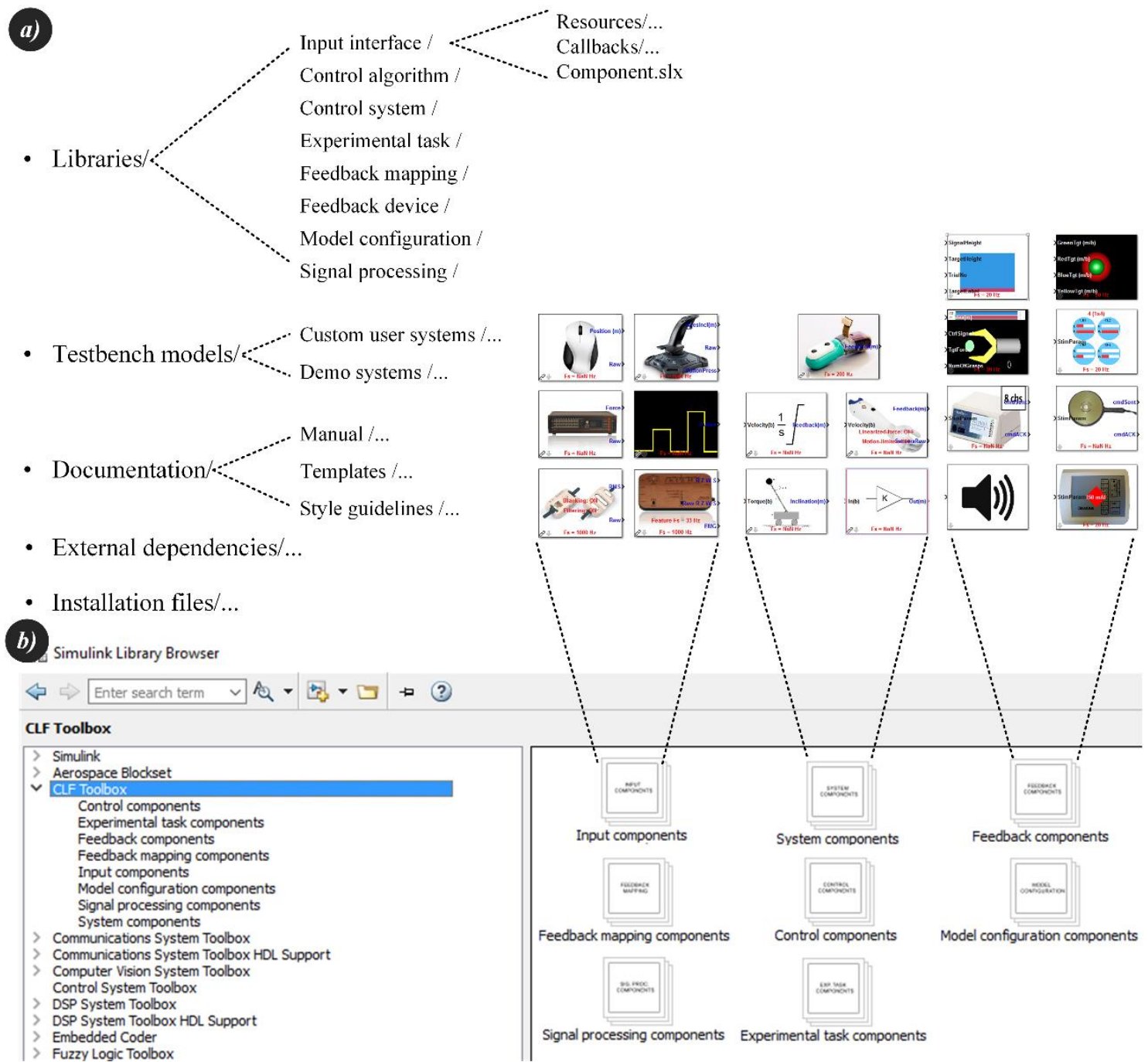


The main features of the CLF (Figure 2.6) are:

- Execution in hardware real-time with sampling frequencies of up to $20 \mathrm{KHz}$ [85], such that the system guarantees that a certain process will finish within the prescribed amount of time.

- High-level user customization, yet transparent system operation. Each block has its own GUI with appropriate customization options. The transparency of execution is achieved by associating the block functions with an intuitive icon. Additionally, the most relevant component settings (e.g., execution frequency) are annotated in red text on the icon itself.

- Interoperability of system components, such that the blocks can be exchanged freely, without breaking the system functionality. This is achieved by utilizing normalized inputs and outputs (I/O signals are always between $[-1,1])$.

- Centralized high-level system management. The CLF takes care of data logging (via DataLogging tags), execution flow, and model configuration (e.g., execution duration, sampling frequency, compiler settings, etc.).

Additionally, the CLF utilizes programming templates/style guidelines and has extensive documentation about the core system features as well as the specific component functionalities.

In an exemplary CLS setup given in Figure 2.6, the user employs a joystick to proportionally control the prosthesis' opening or closing speed by regulating its inclination angle to the left or right, respectively. The experimental task is configured as compensatory tracking of a predefined profile with the generated prosthesis force as the input. This means that the user's task is to match the prosthesis grip force according to the tracking profile. If he is successful, the resulting output of the experimental task block will be close to zero. The output of the experimental task is fed to the information-coding block that maps the sign of the error to one of the two available vibrotactile C2-tactors (residing on ulnar and radial part of the user's lower arm) and the error's amplitude to the intensity of the tactile stimulation. The user will thus feel the error as the intensity of vibration. This simple CLS setup can be used to understand the complex processes behind closed-loop prosthesis control. Namely, by simply exchanging a few blocks: e.g., the real prosthetic hand with the virtual one, the joystick with myoelectric interface, the vibro-tactile with an electro-tactile device or intensity with frequency coding, it would be possible to independently evaluate all contributing 
factors (i.e., the control interface, the control system, the information coding and the feedback modality) on the overall closed-loop performance. Importantly, since they are interoperable and use normalized $\mathrm{I} / \mathrm{O}$, exchanging the blocks within the system is as easy as performing a drag and drop operation from the Simulink Library Browser. Likewise, since the CLF logs the user-selected signals, the scripts used for data analysis can remain virtually unaltered as long as the experimental task does not change; also, the experimental-setup that was used for data acquisition can always be easily reconstructed because the model-specific parameters and settings are automatically saved.

Figure 2.6: An exemplary closed-loop system setup used for evaluating the human performance in steering the prosthesis' force while utilizing tactile feedback. The flash symbols indicates that the additional, component-specific, settings open once the annotated component is double-clicked (customization). Each component has an intuitive icon and displays its most important parameters in red (transparency of operation). The overall CLS model execution is configured within a single block. The model will automatically compile and execute as soon as the START button is double clicked (centralized high-level system management). The data logging block logs the model settings automatically (e.g., component and model parameters) as well as the user-selected signals.

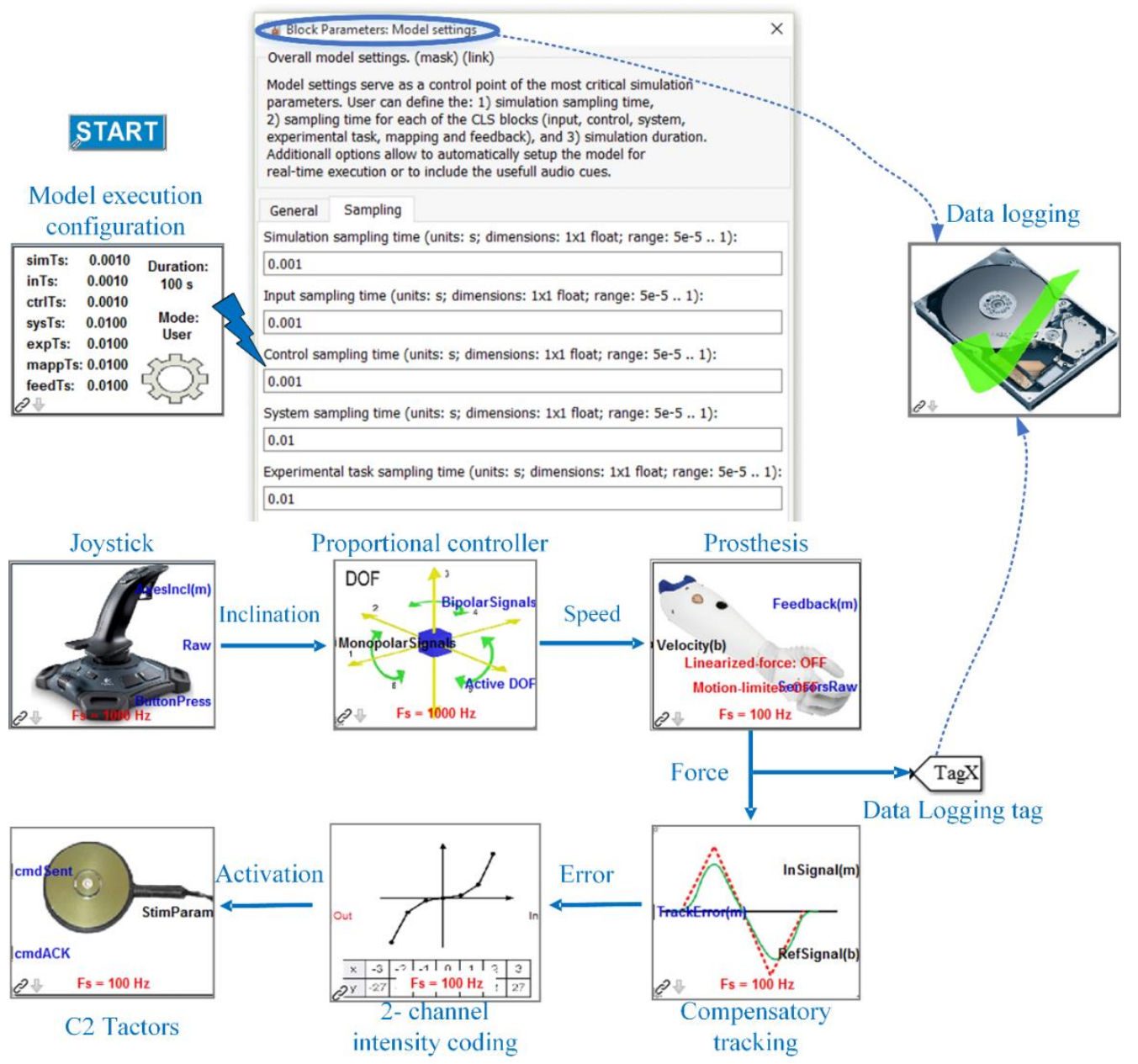




\subsubsection{A novel feedback concept: Biofeedback}

In Appendix 4 [74], it was proposed to feedback the prosthesis control signals, i.e. the generated surface EMG envelope (bioFB), instead of the state of the prosthesis (e.g., force or velocity). The justification behind this approach lies in the fact that SoA myoelectric prostheses are controlled proportionally (as described in the Introduction chapter). That is to say, that the hand closing velocity and thus the resulting grasp force are proportional to the generated myoelectric signal. Therefore, the myoelectric signal anticipates the grasping force, providing time for feedback-driven corrections and since the feedback transmits the latent state of the user (contraction strength) and not the prosthesis outputs, the slow system dynamics do not affect the control. The prosthesis time delays and inertia are outside the biofeedback loop and the online control can therefore be fast and responsive (control signal modulation, Figure 2.7). Consequently, there is more opportunity for predictive feedback-driven corrections and the proprioceptive loop is additionally reinforced.

Figure 2.7: Fundamental differences between EMG biofeedback (bioFB) and force feedback (forceFB) in the context of prosthesis control. During forceFB, the generated grasping force is transmitted to the user only after touch onset, whereas during bioFB the user receives information about the generated EMG online even before the prosthesis reacts. Image adapted from [74].

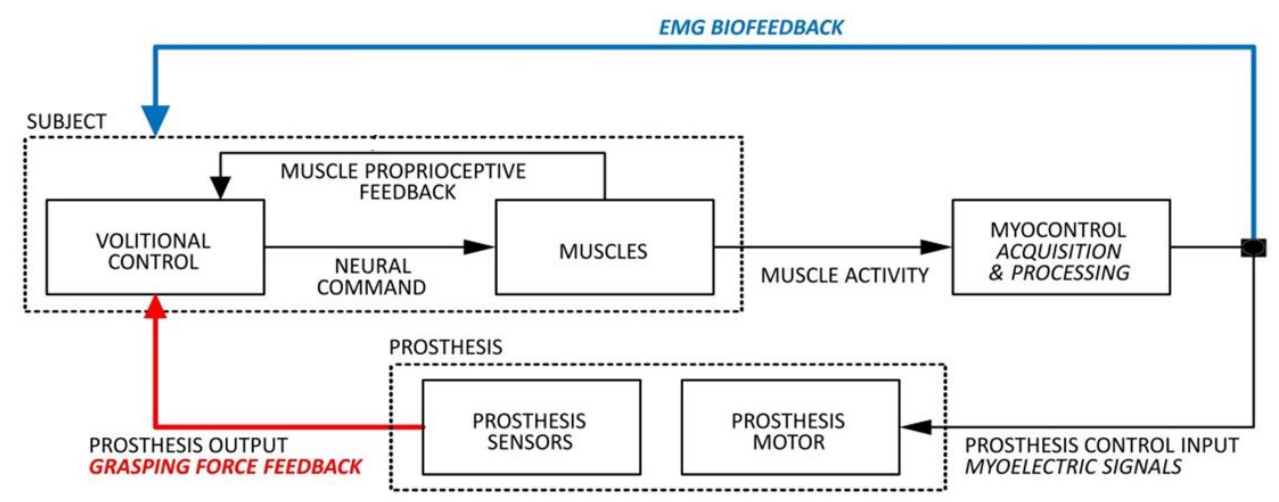

\subsubsection{Experimental setup and evaluation: Biofeedback}

Ten able-bodied subjects ( $23 \pm 3$ yrs.) and two amputees (55 and 43 years) were recruited for the evaluation study described in Appendix 4 [74] (hereafter referred as Study C). The study was designed to test the hypothesis if the biofeedback outperforms force feedback. To this end, two experimental scenarios were evaluated: 1) force generation during repetitive grasping (referred to as routine grasping) and 2) force modulation with reference tracking (referred to as force steering). Each of the two test scenarios was performed using either force- (forceFB) or bio-feedback (bioFB) at a 
time, in a randomized order. The outcome measures were the precision of generated forces (measured as the interquartile range [IQR]) and the root mean square error (RMSE) for routine grasping and force steering tasks, respectively (for more information consult Table 2.3).

The experimental setup was designed in the CLF and comprised: 1) a Michelangelo Hand prosthesis (Otto Bock Healthcare Products GmbH, Vienna, AT), 2) an EMG amplifier (INTEMG, OTBioelettronica, IT), and 3) a standard desktop computer with a 22 " screen. Independent of the experimental scenario the subjects utilized the SoA myoelectric interface in order to grasp a stiff cylindrical object that was positioned and secured between the prosthesis fingers so that the hand grasped it when closed. During the experiment, the prosthesis and the object were detached from the subject and placed in another room in order to isolate the user from the auditory feedback. The prosthesis sensor data (position and force) were sampled internally by the embedded controller $(100 \mathrm{~Hz})$ and then sent to the host PC in order to update the visual feedback displayed on the computer screen. Therefore, the user observed a graphical render of the real prosthesis grasping a real object. The bio- or force-feedback, together with the experimental task, was also rendered within the same graphical interface (Figure 2.8).

Table 2.3: Summary of the experimental evaluations performed in Study C.

\begin{tabular}{|c|c|c|}
\hline & Routine grasping & Force steering \\
\hline $\begin{array}{l}\text { Experimental setup } \\
\& \text { overall task }\end{array}$ & $\begin{array}{l}\text { The subject sits in front of the } \\
\text { computer screen and tries to match } \\
\text { the target force level while closing } \\
\text { the prosthesis in one smooth } \\
\text { movement. Three target levels } \\
\text { were used: } 30 \%, 50 \% \text {, and } 70 \% \text {. }\end{array}$ & $\begin{array}{l}\text { The subject sits in front of the } \\
\text { computer screen and tries to match } \\
110 \text {-s long pseudorandom reference } \\
\text { trajectory comprising a sequence of } \\
\text { increasing and decreasing slopes by } \\
\text { gradually opening/closing the } \\
\text { prosthesis. }\end{array}$ \\
\hline System & \multicolumn{2}{|c|}{$\begin{array}{l}\text { Implemented in the CLF; Closed-loop prosthesis control utilizing SoA } \\
\text { myoelectric interface and visual feedback. }\end{array}$} \\
\hline Subjects & \multicolumn{2}{|c|}{$\begin{array}{l}10 \text { able-bodied subjects ( } 23 \pm 3 \text { yrs.) and two amputees ( } 55 \text { and } 43 \text { years } \\
\text { old). }\end{array}$} \\
\hline $\begin{array}{l}\text { Feedback } \\
\text { conditions }\end{array}$ & $\begin{array}{l}\text { 1. Biofeedback } \\
\text { 2. Force feedback }\end{array}$ & $\begin{array}{l}\text { 1. Biofeedback } \\
\text { 2. Force feedback }\end{array}$ \\
\hline $\begin{array}{l}\text { trials } \\
\text { ion }\end{array}$ & $\begin{array}{l}150 \text { grasping trials }(50 \text { per each } \\
\text { level) }\end{array}$ & Four force tracking trials \\
\hline $\begin{array}{l}\text { Relevant outcome } \\
\text { measures }\end{array}$ & $\begin{array}{l}\text { Interquartile range (IQR): } \\
\text { Measures the dispersion of the } \\
\text { generated forces }\end{array}$ & $\begin{array}{l}\text { The quality of force tracking was } \\
\text { assessed by calculating the root } \\
\text { mean square tracking error (RMSE) } \\
\text { between the generated and the } \\
\text { reference force }\end{array}$ \\
\hline
\end{tabular}


Figure 2.8: Visual scene shown to the subjects during Study $C$ including a snapshot of the screen (a) before contact and (b) after contact. Horizontal bars, red for the flexor and blue for the extensor, showed a continuous feedback about the current level of muscle activity (prosthesis control signals). The semi-transparent blue bar indicated the hand grasping force and the green vertical line the target force level. During routine grasping, the target force was stationary, whereas in the force steering task, it was moving according to the time profile of a reference force trajectory. Note that the biofeedback was shown only during the respective feedback scenario. Image adapted from [74].
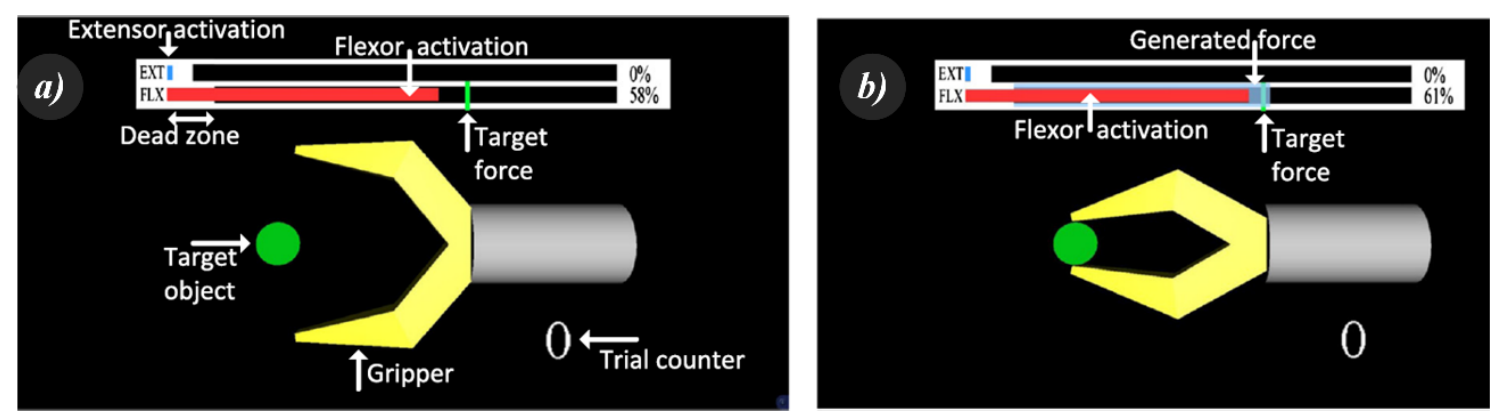

\subsubsection{Practical implementation of the Biofeedback: Google Glass}

As previously described, the biofeedback was experimentally evaluated using the standardized CLF development test bench. This had the advantage of easy system development, data logging, and standardized evaluation. Nevertheless, the biofeedback was rendered visually on a computer monitor which made it difficult to be deployed to the end-user in a practical context (e.g., ADLs).

To address this issue, the presented biofeedback scheme was implemented using a wearable augmented-reality head mounted device (i.e., the Google Glass [86], hereafter the Glass). The Glass integrates smartphone functionalities (e.g., a processor, a battery, $\mathrm{BT}, \mathrm{Wi}-\mathrm{Fi}$, and the ability to run custom-developed apps) with the optical headmounted display (OHMD) and a rudimental user interface (touch, voice commands) which makes it an ideal platform for practical deployment of the augmented-reality biofeedback.

In the current prototype, an app that runs on the Glass has been developed (Figure 2.9). The app connects to the 3-DoF Michelangelo hand prosthesis via the BT interface, fetches the feedback data (i.e., EMG activity and prosthesis states), and displays it on the embedded OHDM in real-time. Since the OHDM display is see-through the user perceives the rendered feedback information as an overlay that augments the real-world (AR feedback). It should be noted that the Glass acts as a feedback device, meaning that it does not influence the prosthesis which uses the SoA two-site myoelectric control with mode switching. In order to overcome the rather limited interaction interface (i.e., 
a single touchpad) and still offer a certain level of user interactivity and customization three different biofeedback representations were developed (Figure 2.9a, b, and c). The user can switch between them by performing a scroll gesture on the Glass touchpad:

- Figure 2.9a: In this representation the two sEMG signals that are driving the prosthesis are rendered in two separate boxes (blue bars). Each of them has a custom-defined dead-zone (dark-gray) and 4 vertical bars that act as references. In addition to the sEMG activity (biofeedback), the prosthesis force (red bar) as well as the currently controlled DoF (box-framed pictures of the palmar, lateral, and rotation) are also displayed.

- Figure 2.9b: Shows the same information as the first render but concatenates the two biofeedback bars into a single one. The resulting representation is thus very similar to a single-axis joystick with two movement directions (left and right).

- Figure 2.9c: Shows the same information as two previous renders but utilizes numbers instead of the bars. The background picture indicates the currently active DoF; The red number indicates the current force, while the bottom number indicates the activity of the corresponding sEMG channel by changing its color (blue or green for flexor or extensor, respectively).

Figure 2.9: Biofeedback implementation on the Google Glass. The Glass connects to the Michelangelo prosthesis via BT and renders the acquired feedback on the OHDM. The user can switch between different representations by performing a scroll gesture on the touchpad.

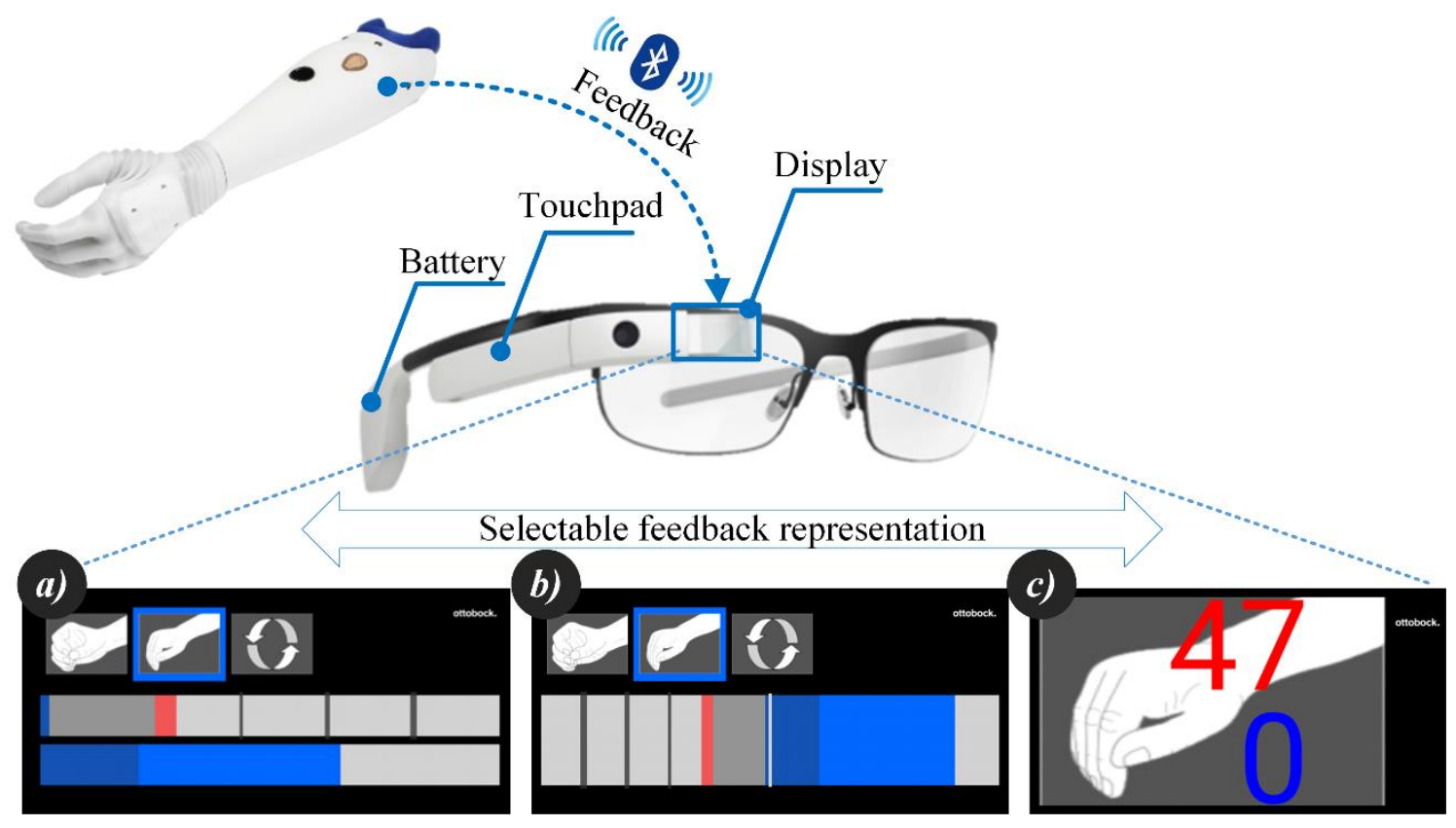




\subsection{Data Analysis}

In all three studies, the data were analysed using Matlab (MathWorks, Natick, US-MA) software package and Statistics Toolbox. The analysis was performed separately for the able-bodied and the amputee group. Since in none of the studies the proportion of amputees was large enough, the data-analysis outcomes for amputee subjects were just reported, but not tested for statistical significance.

\subsubsection{Prosthesis Control (Studies A and B)}

In Study A (used the first CASP prototype), the analysis was performed by calculating the performance outcomes (SEE, PSR, SFR, and TAR) for each grasping trial and then pooling respective trial outcomes together across all subjects for each of the conditions separately (AUTO and SEMI). The two-sided pairwise t-test for dependent samples (repeated measures) was used for pair-wise comparison between the conditions (AUTO and SEMI). A p-value of 0.05 was selected as threshold for statistical significance.

In Study B (used the CASP), the analysis was performed by calculating the performance outcomes (TTG, OEE, Shoulder Euler Angles) for each grasping trial and then pooling respective trial outcomes together across all able-bodied subjects, separately for each of the conditions (MAN1/2/3 and CASP) and each experimental session (training and evaluation). Friedman test was used to assess statistically significant differences within the group of conditions in the evaluation session, since the data did not pass the test of normality (Liliefor test). For pairwise comparisons, Tukey's honestly significant difference criterion was applied. Finally, to compare the same condition between training and testing, Wilcoxon signed-rank tests were employed. A p-value of 0.05 was selected as threshold for statistical significance.

\subsubsection{Prosthesis Feedback (Study C)}

The analysis was performed by calculating the performance outcomes of each trial (IQR or RMSE) and then pooling respective trial outcomes together across all able-bodied subjects, separately for each of the experimental scenarios (routine grasping, force steering) and conditions (bioFB, forceFB). All the results were reported as normalized forces, in fractions or percent, i.e., $100 \%$ corresponded to the maximum force of the prosthesis $(\sim 100 \mathrm{~N})$. For the routine grasping scenario, the Bartlett multiple-sample test for equal variances was applied to test for statistically significant differences in dispersion (IQR) within the conditions overall, followed by Ansari-Bradley two-sample 
test with Bonferroni correction for pairwise comparisons of the force variability between the conditions. The statistical analysis for the RMSE outcome of the force tracking task was performed using Wilcoxon signed rank test, as the data did not pass the normality test (one sample Kolmogorov-Smirnov). The threshold for statistical significance was adopted at $\mathrm{p}<0.05$. 


\section{RESULTS}

In the following paragraphs the results obtained from the two studies presenting the novel CASP system (Studies A and B, Appendix 1-2, [71], [72]) and the one study presenting the biofeedback (Study C, Appendix 4, [74]) will be shortly disseminated. The results are reported in the format: mean \pm standard deviation and are presented separately for able-bodied (figures and text) and the amputee subjects (text only).

\subsection{Prosthesis Control (Studies A and B)}

In Studies A and B, the CASP system was evaluated on a variety of objects (30 in total) in different positions and orientations, which presents a good database for evaluating the performance of the employed object modeling algorithm. The object size estimation error (SEE, Study A) and the orientation estimation errors (OEE, Study B) were $0.75 \pm$ $1.1 \mathrm{~cm}$ (mean \pm standard deviation) and $9 \pm 5^{\circ}$, respectively.

\subsubsection{Study A}

Figure 3.1 summarizes the performance of the first CASP prototype for able-bodied subjects (PSR, TFR, and SFR) in the two test scenarios (AUTO and SEMI) which included $520(13$ subjects $\times 2$ series $\times 20$ objects $)$ and 260 trials $(13$ subjects $\times 1$ series $\times 20$ objects), respectively.

When the manual control was inactive (AUTO operation mode), the preshape success rate (PSR) was 79\%. As soon as the user was allowed to correct for eventual errors (SEMI operation mode), the PSR improved significantly by $16 \%$ and reached $95 \%$ (Figure 3.1a). 
The task accomplishment outcome measures (TAR, SFR) are shown in the pie charts in Figure 3.1b. During the AUTO condition, the task was successfully completed in $73 \%$ of trials. Successful completion of the task increased significantly to $81 \%$ in the SEMI condition. This improvement was due to a significant reduction of the system-induced task failures rate (SFR), which dropped from $10 \%$ to only $3 \%$, whereas the myoelectric control induced failures remained virtually unchanged $(16-17 \%)$.

Figure 3.1: Study A: a) Summary results for the CASP preshape success rate (PSR). b) Task accomplishment/failure rate (TAR/SFR) for the two operation modes: without (AUTO) and with manual user control (SEMI). Statistically significant differences are denoted by a star (*, $p<0.05)$. Image adapted from [71].

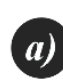

Preshape performance rate (PSR)

(able-bodied subjects)

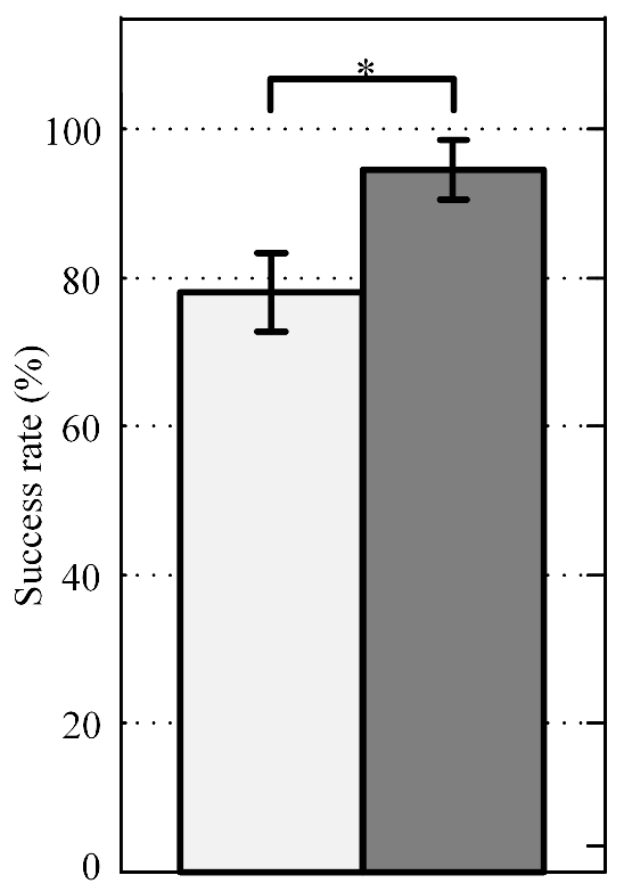

b) Task accomplishment/failure rates (TAR/SFR)

(able-bodied subjects)

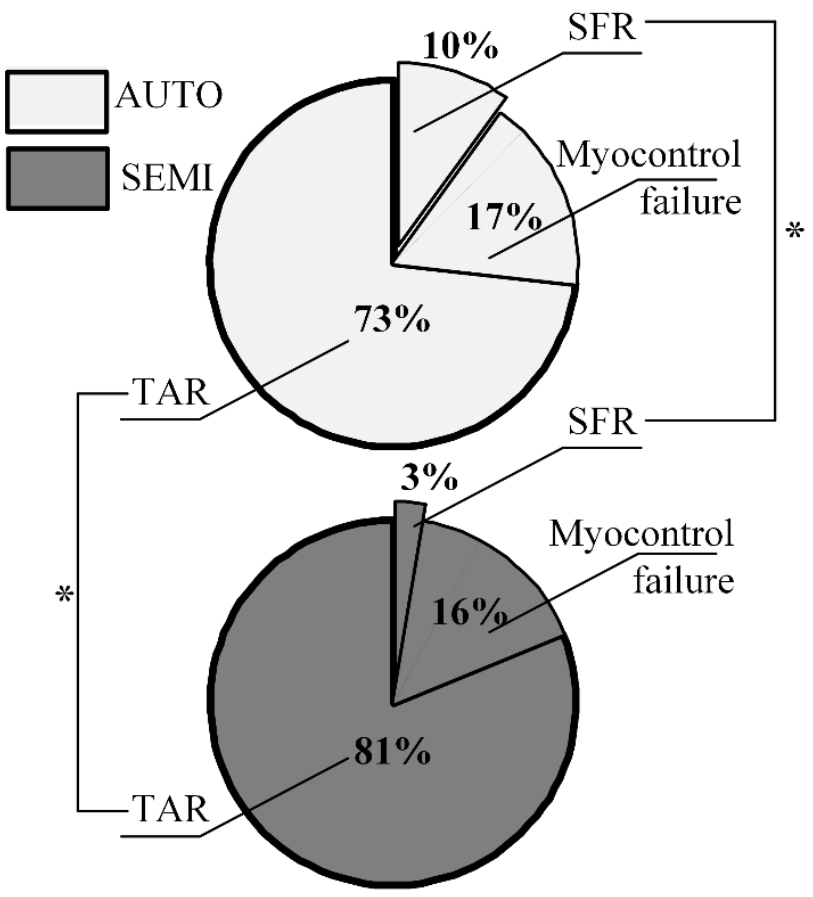

\subsubsection{Study B}

In total, 1360 grasping trials (10 subjects $\times 2$ days $\times 4$ conditions $\times 17$ trials) were performed by able-bodied subjects. One amputee subject performed an additional 136 grasping trials ( 1 subject $\times 2$ days $\times 4$ conditions $\times 17$ trials). All grasping trials were allocated evenly between the four control conditions (MAN1-3, and CASP). Figure 3.2 summarizes the results for able-bodied subjects.

The average time to grasp (TTG) for each of the four test conditions and training and evaluation sessions is shown in Figure 3.2a. During the evaluation session, the TTG in the manual control scenarios (MAN1-3) increased consistently with the number of controllable DoFs, i.e., it was $3.7 \pm 1$ s for MAN1, $4.3 \pm 1.7$ s for MAN2, and then 
increased substantially to $11.2 \pm 4.1 \mathrm{~s}$ in MAN3. The differences were statistically significant between all MAN conditions. The TTG with CASP was $5.9 \pm 1.9 \mathrm{~s}$, which was slower than in MAN1 and MAN2 but substantially faster than in MAN3. Additionally, there was virtually no improvement between the training and evaluation sessions with CASP, contrary to the MAN2 and MAN3 conditions, which improved with training.

The recorded shoulder joint angles in MAN1 and 2 differed significantly in abduction and external rotation compared to the conditions with manual (MAN3) or automatic (CASP) rotation control. Between MAN1 and 2, the angles were similar, which also held for MAN3 versus CASP (for exact values please refer to the published article in Appendix 2, [72]). Representative shoulder configurations for MAN1 and CASP are depicted in Figure 3.2b when a cup was positioned horizontally (left picture) and vertically (right picture). Since the wrist rotation was inactive, in MAN1 the user had to perform extensive compensatory movements consisting of either shoulder abduction and external rotation (left picture) or adduction and internal rotation (right picture) in order to orient the hand appropriately for grasping the object. On the other hand, there were no such over-extensive movements when using CASP and in that case the shoulder angles remained virtually unaffected by the object orientations, since the automatic control adjusted the hand orientation accordingly, using the active wrist joint. Similar trends were also observed in the amputee subject where the average TTG was $2.5 \pm 1 \mathrm{~s}, 4.7 \pm 1.8 \mathrm{~s}, 10 \pm 2.2 \mathrm{~s}$, and $5.5 \pm 1.8 \mathrm{~s}$ for MAN1-3 and the CASP conditions respectively. The subject successfully learned how to use the system and, although he was an experienced user of a classic 1-DoF myoelectric prosthesis, the results were similar to the ones obtained for able-bodied subjects. The CASP system was approximately twice as fast as manual control for the same number of DoFs (MAN3). 
Figure 3.2: Study B: a) Summary results for the average time to grasp (TTG) an object across conditions (MAN1-3, CASP) and experimental sessions (training, evaluation). The statistically significant differences are denoted by a star (*, $p<0.05)$; the symbol ' $C$ ' indicates that the difference exists across all conditions that were performed within the same experimental session. b) 3D model showing the arm positions recorded shortly before the object was grasped. An object placed horizontally (left) and vertically (right) was grasped using MAN1 and CASP control schemes. Image adapted from [72].

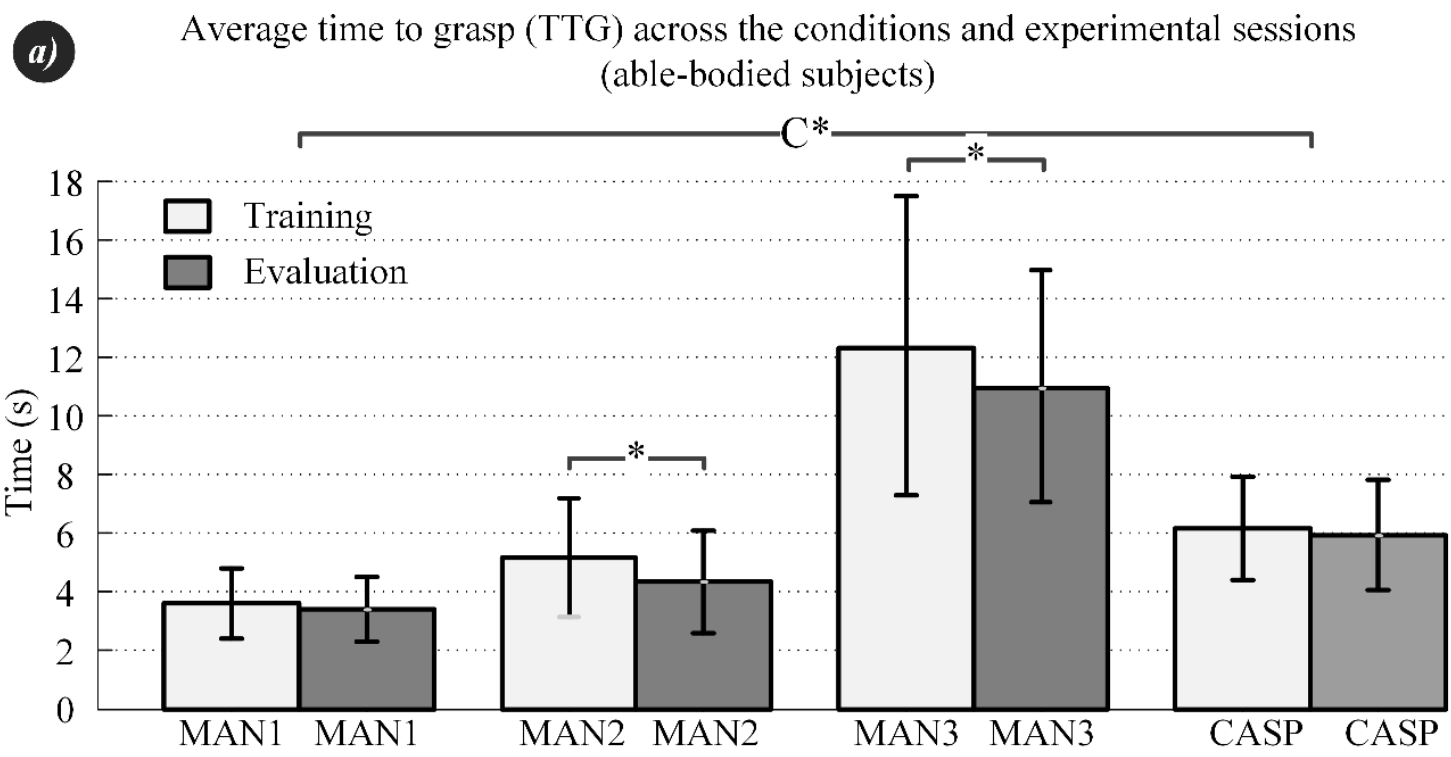

b) Representative arm configurations recorded during MAN1 and CASP conditions
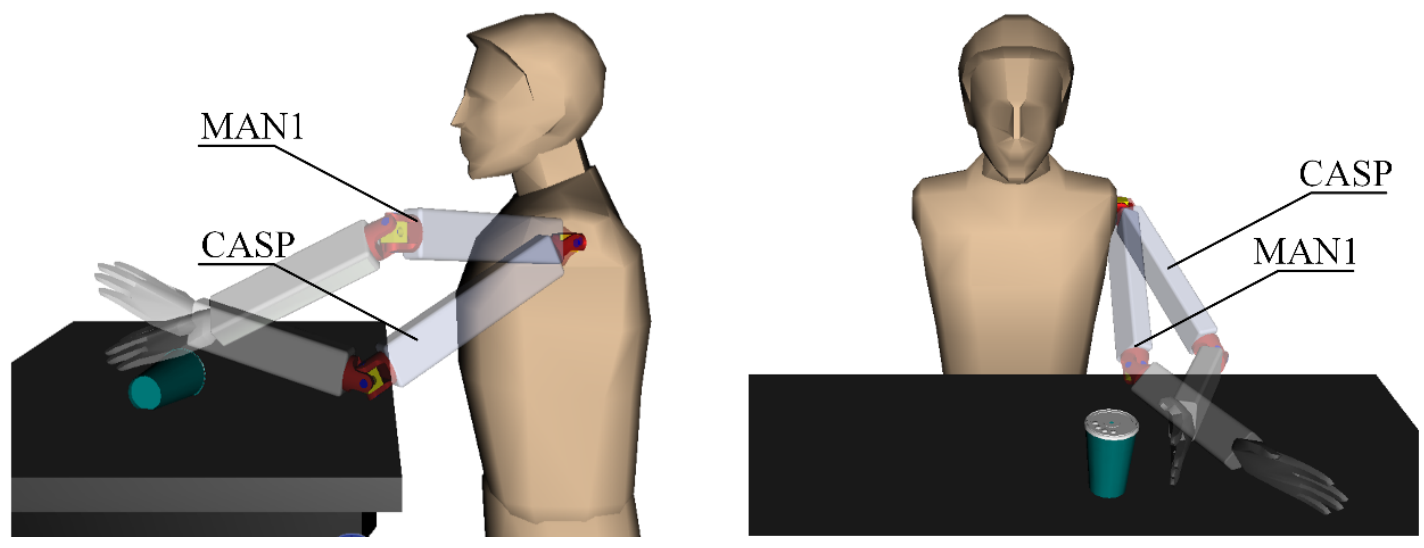

\subsection{Prosthesis Feedback (Study C)}

Summary results for ten able-bodied subjects and all conditions (force- and biofeedback) and experimental tasks (routine grasping and force steering) are presented in Figure 3.3.

Providing EMG biofeedback significantly improved the consistency in generating grasping forces at all three force levels (Figure 3.3a). Without EMG biofeedback, the IQRs were $10 \%, 14 \%$ and $16 \%$ for the target force of $30 \%, 50 \%$, and $70 \%$, respectively, 
and they were approximately twofold lower when EMG biofeedback was transmitted (i.e., $6 \%, 6 \%$, and $7 \%$, respectively). Likewise, with the force feedback, the force variability increased significantly for the higher target forces (forceFB [30\%] vs. forceFB [50\%] and forceFB [70\%]), which was not the case for the EMG biofeedback condition.

Figure 3.3:Sudy C: a) Routine grasping task performance in two feedback conditions (forceFB and bioFB) and at three target force levels (30, 50, and 70\%). Boxplots depict the median (red line), interquartile range (blue box), maximal/minimal values (whiskers) and outliers (red crosses). Dashed gray lines are the target force levels. $b$ ) Force-tracking task performance. The toot mean square tracking error is given for two conditions (forceFB, and bioFB). Statistically significant differences are denoted by a star (*, $p<0.05)$. Image adapted from [74].
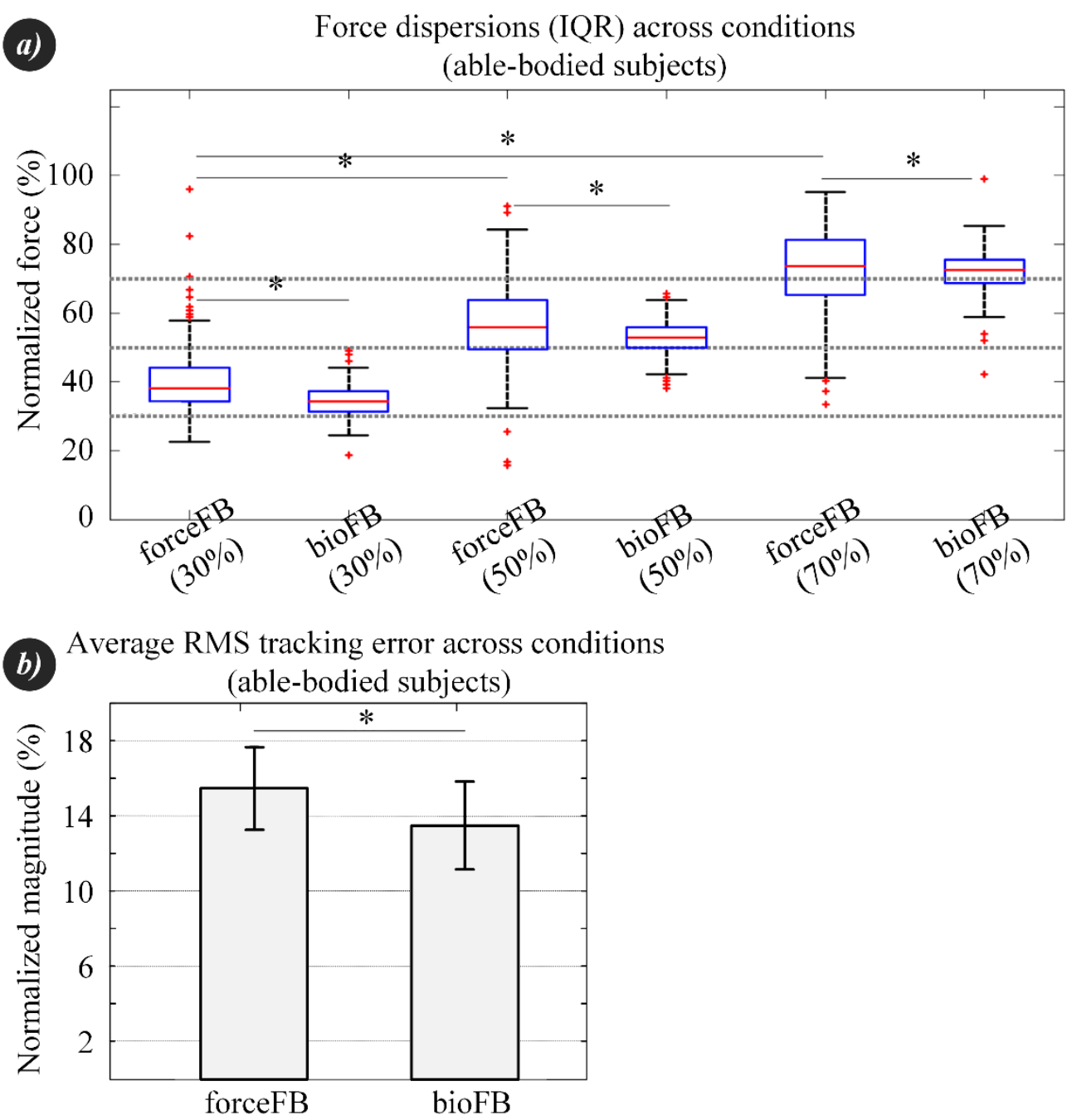

During the force steering task, providing EMG biofeedback reduced tracking error (Figure 3.3b). The decrease was modest but statistically significant $(15.5 \pm 2 \%$ for forceFB vs. $13.5 \pm 2 \%$ for bioFB, $\mathrm{p}<0.001)$.

The results for the two amputee subjects demonstrated a similar trend as in able bodied 
subjects. The provision of the EMG biofeedback reduced the IQR of the generated forces from $13 \%, 9 \%$ and $16 \%$ for forceFB to $9 \%, 8 \%$, and $10 \%$ for bioFB for the target forces of $30 \%, 50 \%$, and $70 \%$, respectively. The relative improvement was, however, less than in able-bodied subjects. The same trend can be observed in force tracking task: The tracking errors decreased from $16.8 \%$ and $18.8 \%$ in forceFB to $13 \%$ and $17.8 \%$ in bioFB for amputee 1 and 2, respectively. 


\section{DISCUSSION}

In this dissertation the contributions from four peer-reviewed publications addressing the topics of prosthesis control and feedback were presented. Specifically, in the publications presented in Appendix 1-2 ([71], [72]), the novel context and user aware system for prosthesis control (CASP) was developed and evaluated in 23 able-bodied and 1 amputee subject (Studies A and B). Likewise, in the publications presented in Appendix 3-4 ([73], [74]), closing the loop with prosthesis was addressed by presenting a comprehensive closed-loop framework (CLF) in which the novel biofeedback (bioFB) concept for enhancing myoelectric prosthesis control was developed and evaluated in 10 able-bodied and 2 amputee subjects (Study C). Hereafter, the relevant results and the methods from the four aforementioned publications will be summarized and discussed.

\subsection{Prosthesis Control (CASP, Studies A and B)}

The CASP system introduced a novel paradigm for semi-autonomous (shared), context dependent and reactive prosthesis control that infers the user intensions in real-time and adapts to them accordingly by fusing and analyzing the data from a variety of external sensors; i.e., sEMG, IMU, camera, embedded prosthesis force, and position encoders. The novel functions of CASP have been also patented in [77].

The experimental setup described in section 2.1.3 was designed to evaluate the system, during the typical object grasp and transport task, on two levels. One is the conceptual, presented in Study A, which was used to assess the overall viability and accuracy of the semi-autonomous control. The other one functional, presented in Study B, assessed the performance and functionality of the system as a whole, specifically in respect to the 
SoA myoelectric control.

The results depicted in Figure 3.1 indicate that the semi-autonomous prosthesis control concept (SEMI), in which the user and the autonomous CASP controller share the responsibility, is not only feasible but also a preferable mode of control compared to a fully autonomous one (AUTO). This is demonstrated by statistically significant increases in both the preshape accuracy (PSR) and the task accomplishment rates (TAR) (AUTO vs. SEMI). Namely, in the SEMI mode the CASP operation was not flawless but the subjects were able to manually correct most of the system-induced errors which resulted in reaching virtually $100 \%$ in PSR. Therefore, the overall feasibility of the semi-autonomous control concept is confirmed by the fact that only $3 \%$ of the task failures were caused by system-induced errors. This increase was only partly translated to an increase in the overall TAR. The reason behind this was that the task accomplishment was strongly influenced by the quality of myoelectric control which was used in both conditions (AUTO, and SEMI) for triggering specific prosthesis operations (e.g., closing/opening) and thus remained responsible for steady $16 \%$ of task failures.

Observing Figure 3.2 it can be noted that the single-DoF control (MAN1) was the simplest scenario, in which the subjects only had to reach for the object and close the hand, resulting in the smallest TTG overall. However, the subjects had to employ compensatory strategies characterized by excessive shoulder movements in order to accommodate for the lack of active DoFs. In MAN2, the control scheme increased in complexity, as the subjects had to perform a co-contraction to switch between the grasp types. The TTG increased slightly, however, the addition of a novel grasp-function did not change the reaching strategy, and the same compensatory movements were still present. Only in MAN3 and CASP, in which the wrist could be also actively rotated, the overall shoulder angles displayed a significant reduction, especially in abduction and external rotation. However, since the subjects had to perform tiresome cocontractions several times in a single grasping trial the TTG in MAN3 increased consequently by a factor of $3 / 2$ with respect to MAN1/2. In contrast, the CASP system is somewhat slower than MAN1 and 2 but results in grasping strategies and arm configurations that are similar to those employed in MAN3, with the benefit that it is substantially faster than the latter. Therefore, when using all available prosthesis DoFs, the CASP outperforms the manual myoelectric control significantly. Furthermore, the CASP system was easy to adopt and use, which is demonstrated by the fact that there 
was no significant improvement in performance between the two experimental sessions (training and evaluation were the same), in contrast to the MAN3 condition, which displayed a training effect (evaluation phase better than training). Importantly, the results obtained from an amputee likewise indicated the same trend.

Concerning the performance, the CASP system was able to respond within a second to a user-generated trigger command. The response delay included trigger detection, information acquisition, fusion, and analysis (e.g., object modeling and calculation of possible hand postures), as well as the transfer of the appropriate preshape commands to the prosthesis. The overall precision of the sensor-fusion algorithm, and specifically its core-part designated for object modeling, was likewise reasonably good. It showed on average errors less than $1 \mathrm{~cm}$ and 10 degrees' in object size (SEE) and orientation estimation (OEE), respectively.

In summary, it has been demonstrated that by integrating artificial proprioception and vision with the voluntary (myoelectric) control an innovative prosthesis control paradigm can be effectively placed into function. In its current implementation, the CASP system could automatically and simultaneously control prosthesis preshape, aperture, and orientation (grasp planning) by utilizing only a rudimental user input (i.e., a simple myoelectric trigger). Additionally, it used inertial sensing to detect changes in the hand-to-object approach strategy (i.e., the user intentions) and reacted to them in real-time by readjusting the aforementioned prosthesis parameters accordingly (reactive control). In the two studies it has been experimentally demonstrated that this concept cannot only function in practice, but also bring visible performance benefits in comparison to SoA myoelectric control. It can even be hypothesized that these automatic functions (i.e., grasp planning and reactive control) could potentially decrease the user's cognitive effort by providing him the opportunity to focus on the task rather than on how to steer the prosthesis.

\subsection{Prosthesis feedback (CLF, Biofeedback, Study C)}

The closed-loop development framework, presented in Appendix 3, was utilized to design and evaluate a variety of different CLS experimental setups ([69], [73], [74], [84]). The CLF contains many ready-to-use components that can be combined and exchanged in an arbitrary fashion, which allows for effortless system (re)configuration. Moreover, the overall structural and executional transparency make the systems designed in the CLF easy to use and debug. 
The novel biofeedback approach, presented in Appendix 4, can be regarded as a simple feedforward simulation of a prosthesis since it provides the subject with an estimate (i.e., prediction) of the command output (e.g., grasping force). In general, this allows the subject to adjust the current online command even before the outcome is generated.

Study $\mathrm{C}$ was designed to test the overall biofeedback approach feasibility. Therefore, the feedback was provided using an ideal interface (visual bar). At first, the visual feedback might seem impractical and difficult to deploy, but in the light of the recent technological advancements in the field of wearable AR devices (e.g., Google Glass) this implementation becomes not only feasible, but also very appealing and practical, as demonstrated in chapter 2.2.4. Nevertheless, the same approach could be implemented using electro- or vibro-tactile stimulation by utilizing spatial, intensity, or frequency coding, as demonstrated in [84]. During the evaluation, the biofeedback was used to predictively control the prosthesis grasping force in two experimental tasks: 1) routine grasping and 2) force tracking. The performance outcomes (IQR, RMSE) in biofeedback scenario (bioFB) were then compared to the ones achieved using the "classical" feedback loop, which relied on force feedback (forceFB) only.

The results depicted in Figure 3.3 demonstrate the superiority of biofeedback to the “classical" feedback loop. All able-bodied as well as amputee subjects demonstrated significant performance increase during the bioFB scenario across both experimental tasks (i.e., smaller force dispersions, and tracking errors). The practical implications of these results are fourfold. The quality of force steering assessed through RMSE is relevant for object holding and manipulation (e.g., squashing a tooth-paste). In practice, unilateral amputees accomplish such sensitive tasks most often using a healthy hand, due to the poor controllability and other limitations [87], which makes the improvements achieved with biofeedback even more relevant. The control reproducibility, assessed during the routine grasping task is yet another important factor that influences the overall prosthesis acceptance. The overall uncertainty of the myoelectric control channel [88] makes this very challenging to achieve in everyday application. On the other hand, the inclusion of biofeedback demonstrated that both the overall accuracy and precision (IQR) of force generation can be significantly increased. Opposed to the "classical" feedback approach, the biofeedback implementation doesn't require the integration of additional sensors into the prosthesis (i.e., force or position encoders). The standard EMG electrodes, which are already a standard part of every myoelectric prosthesis, are all that is necessary [84]. Finally, the concept of 
biofeedback, although demonstrated for single-DoF SoA myocontrol, could be generalized and extended to any number of functions or myoelectric control algorithms. For example, in a practical electro- or vibro-tactile feedback interface, when using an advanced pattern recognition/regression system, the classifier outputs could be communicated to the user by using a mixture of spatial and intensity coding. Here, the spatial information would indicate the classifier-state (i.e., active DoF), while the intensity of the stimulation would correspond to the active DoF velocity.

\subsection{Future work: Sensor-Fusion with AR}

In the past decades prosthesis control has been based almost exclusively on a single type of sensor, namely, a bio-amplifier detecting the electrical muscle activity. However, it is well known from robotics applications that a number of other sensors can be useful for control of reaching, grasping, and manipulation [59]. Similar could be said for the feedback pathway, where the SoA approaches often fail to prove the functional benefits of the feedback [35]. The present work starts from these insights and proposes the integration and fusion of additional sensors into a prosthetic device (CASP system), in order to extend the overall usability, ease of use, and intuitiveness of the employed feedforward MMI. Moreover, it also demonstrates the usage and benefits of novel feedback paradigms and interfaces (biofeedback and AR).

The next logical extension of the presented work would be a combination of the two concepts, namely multimodal sensor-fusion and biofeedback, into a single selfcontained system. This advanced system could integrate both semi-autonomous context-dependent control as well as the intuitive biofeedback together with the SoA myoelectric interface. One possible technical implementation could be the usage of head-mounted AR glasses that integrate advanced RGB-D imaging (e.g., Microsoft HoloLens or Meta Spaceglasses [89], [90]). In this implementation the AR could be used to close the loop and communicate system states (e.g., current DoF) or relevant feedback information to the user (e.g., muscle activation - biofeedback). Moreover, since the AR is rather intuitive (in comparison to cutaneous tactile feedback interfaces), transmission of highly-complex feedback information, such as a full 3D AR render of the prosthetic hand, would also be possible. One proof-of-concept implementation of such system is given in [71], where in addition to the CASP system (in its very first iteration) AR was used to communicate the overall machine states (e.g., object targeting) and prosthesis configuration (i.e., grasp type and aperture) to the user. In 
addition to this, a series of tests were conducted in order to validate that the user could comprehend and utilize the AR feedback information to control and steer the overall system behavior in real-time.

The concept of context-dependent prosthesis control could be extended even further by harvesting the possibilities available through the recent technological advancements. The envisioned future of upper limb prosthetics is to be found in Figure 4.1 and explained in greater detail in the text below.

Figure 4.1: The envisioned future of upper limb prostheses. The user wears an array of devices that communicate wirelessly in order to fuse and process multimodal sensory information. The smart-phone acts as the central processing unit that communicates with a cloud in order to perform computationally intensive operations or update the map of the user's environment. The smartglasses integrate the RGB-D camera together with the see-through AR display thus acting as an artificial exteroception and feedback interface. The smartwatch/smartband is worn around the unaffected arm and provides rudimentary feedback to the user (notifications) as well as the artificial proprioception to the overall control system.

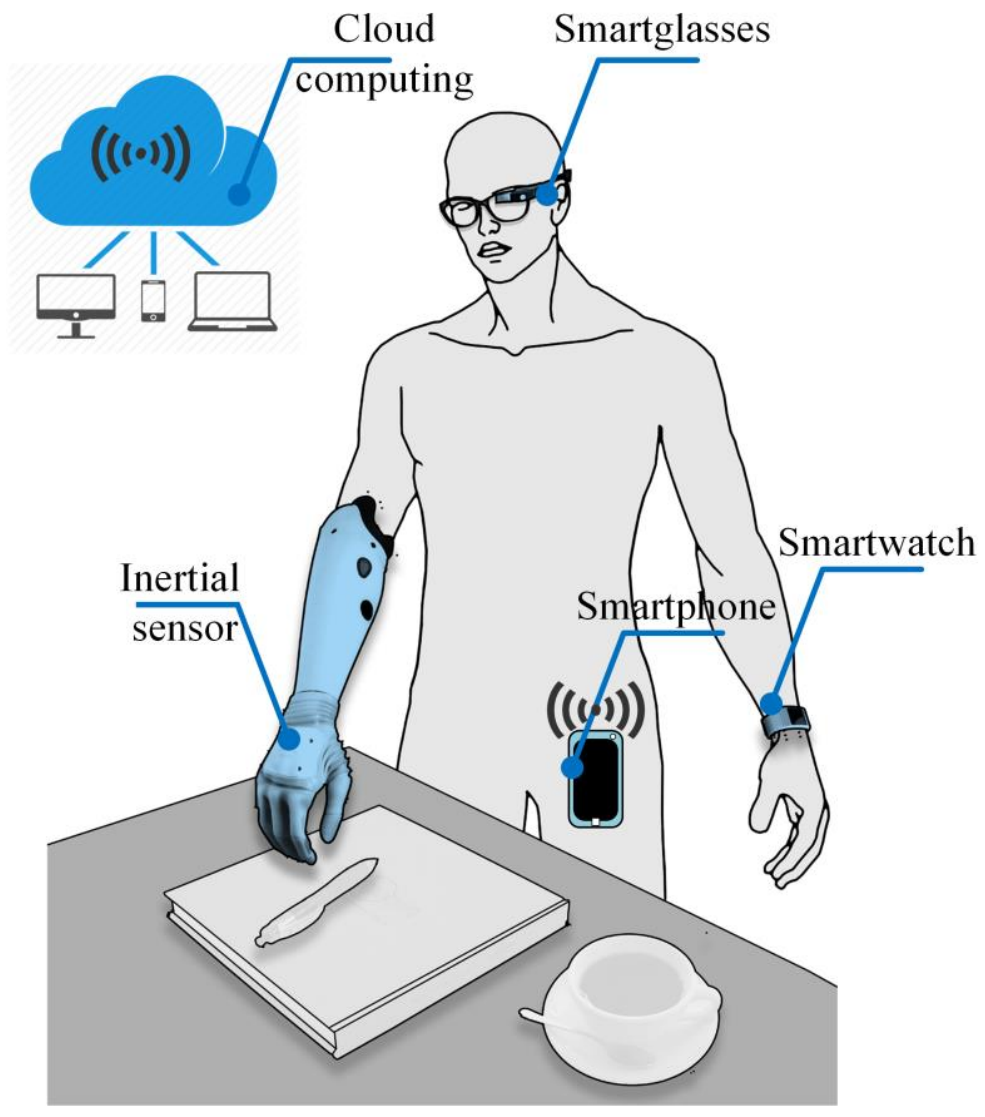

Wearable smart-gadgets such as smart-phones, smart-watches, smart-bands, and smartglasses that all have an array of inbuilt sensors (e.g., cameras, inertial sensing) and feedback interfaces (e.g., sound, vibration, display) could be utilized to better understand the context of prosthesis usage (e.g., via location tracking), provide feedback 
to the user (e.g., a buzz notification on a smart-watch, a display on the smart-glass) or even perform heavy-duty processing (e.g., on a smartphone).

Yet another technological breakthrough that could be used in the context of prosthesis control is cloud computing. Similar to modern-day voice-recognition algorithms (e.g., Google Now, Apple Siri, or Microsoft Cortana) that extract features locally (e.g., on the cell-phone) but then go on a cloud in order to perform the feature matching, computer vision systems could do just the same, thus offering virtually unlimited computing power in a compact form. This could be used to integrate complex computer-vision algorithms that allow for simultaneous user localization, environment recognition, and mapping [91], [76] which in turn could provide rich contextual information (e.g., the user is in front of his work desk and reaches out for a pen, Figure 4.1). In an exemplary application the 6-DoF location of the prosthesis could be inferred through inertial sensing and computer vision and used together with depth reasoning to focus on the part of the scene where a potential interaction can occur. The structure of the scene could be analyzed to segment and model static and moving objects and support surfaces. The object detection and analysis could be handled using a fallback approach. Namely, the system could integrate a database of daily life object models but if the objects are not found in the database they will be represented with their most similar known representative. Additionally, the tracking of the contralateral hand (e.g., via a smartwatch) could also be implemented, in interaction with objects in the environment as well as with the prosthesis, and fused with other proprioceptive estimations. Therefore, the prosthesis could be controlled to adapt to the contralateral hand and the object, thereby supporting bimanual tasks (e.g., grasping a cup with the unaffected hand and transporting it to the prosthesis).

In addition to the cloud-based computer vision and smart-gadgets artificial intelligence based on neural-networks, fuzzy-logic, or deep-learning algorithms could also be utilized to implement concepts such as learning by observation and demonstration. Namely, the system could leverage multimodal sensor data to learn about the environment and adapt to the user and likewise compare its automatic decisions to the user control, detecting when the user selected a different grasping strategy with respect to what the system has planned (learning by observation). Alternatively, the user would be able to explicitly activate the learning function, configure the prosthesis and grasp the object, while the system observes this action mapping the grasping strategy to the model of the object (learning by demonstration). 
Finally, it should be noted that the work presented in this thesis is not designed to replace the SoA upper limb prosthetic interfaces per se, but rather to enhance and endow them with new functions that would make them more intuitive, robust, understandable, and finally effortless for their end-user. 


\section{REFERENCES}

[1] C. L. MacKenzie and T. Iberall, "The Grasping Hand," Adv. Psychol., vol. 104, p. 482, 1994.

[2] K. Ziegler-Graham, E. J. MacKenzie, P. L. Ephraim, T. G. Travison, and R. Brookmeyer, "Estimating the prevalence of limb loss in the United States: 2005 to 2050.," Arch. Phys. Med. Rehabil., vol. 89, no. 3, pp. 422-9, Mar. 2008.

[3] T. R. Dillingham, L. E. Pezzin, and E. J. MacKenzie, "Limb amputation and limb deficiency: epidemiology and recent trends in the United States.," South. Med. J., vol. 95, no. 8, pp. 875-83, Aug. 2002.

[4] P. L. Ephraim, T. R. Dillingham, M. Sector, L. E. Pezzin, and E. J. MacKenzie, "Epidemiology of limb loss and congenital limb deficiency: a review of the literature," Arch. Phys. Med. Rehabil., vol. 84, no. 5, pp. 747-761, May 2003.

[5] L. Löffler, Der Ersatz für die obere Extremität: die Entwicklung von den ersten Zeugnissen bis heute. 1984.

[6] E. A. Biddiss and T. T. Chau, "Upper limb prosthesis use and abandonment: a survey of the last 25 years.," Prosthet. Orthot. Int., vol. 31, no. 3, pp. 236-57, Sep. 2007.

[7] D. W. Dorrance, "Artificial hand.," US1042413 A, 29-Oct-1912.

[8] A. Fougner, O. Stavdahl, P. J. Kyberd, Y. G. Losier, and P. A. Parker, "Control of upper limb prostheses: terminology and proportional myoelectric control-a review.," IEEE Trans. Neural Syst. Rehabil. Eng., vol. 20, no. 5, pp. 663-77, Sep. 2012.

[9] R. M. Enoka and J. Duchateau, "Physiology of Muscle Activation and Force Generation," in Surface Electromyography: Physiology, Enigineering and applications, 2nd ed., i., R. Merletti and D. Farina, Eds. Ieee Computer Soc Pr, 2015, p. 22.

[10] H. G. Näder and L. Milde, OTTO BOCK Prothesen-Kompendium. Prothesen für die obere Extremität. 2011. 
[11] "Touch Bionics, Inc. i-Limb Ultra Revolution data sheet." [Online]. Available: http://www.touchbionics.com/sites/default/files/i-

limb_ultra_revolution_datasheet.pdf. [Accessed: 12-Apr-2015].

[12] "Otto Bock HealthCare GmbH, Michelangelo ${ }^{\circledR} . "$ [Online]. Available: http://www.ottobock.com/cps/rde/xchg/ob_com_en/hs.xsl/49464.html. [Accessed: 09-Feb-2015].

[13] "Motion Control, Inc., U3 Arm Myoelectric Prosthetic." [Online]. Available: http://www.utaharm.com/ua3-myoelectric-arm.php. [Accessed: 09-Nov-2015].

[14] R. S. Johansson and K. J. Cole, "Sensory-motor coordination during grasping and manipulative actions.," Curr. Opin. Neurobiol., vol. 2, no. 6, pp. 815-823, 1992.

[15] I. Saunders and S. Vijayakumar, "The role of feed-forward and feedback processes for closed-loop prosthesis control," J. Neuroeng. Rehabil., vol. 8, no. 1, p. 60, 2011.

[16] J. Hermsdörfer, Z. Elias, J. D. Cole, B. M. Quaney, and D. a Nowak, "Preserved and impaired aspects of feed-forward grip force control after chronic somatosensory deafferentation.," Neurorehabil. Neural Repair, vol. 22, no. 4, pp. 374-384, 2008.

[17] R. N. Lemon, "Functional properties of monkey motor cortex neurones receiving afferent input from the hand and fingers," J. Physiol., vol. 311, pp. 497-519, 1981.

[18] I. Rosén and H. Asanuma, "Peripheral afferent inputs to the forelimb area of the monkey motor cortex: Input-output relations," Exp. Brain Res., vol. 14, no. 3, pp. 257-273, 1972.

[19] D. F. Lovely, "Signals and Signal Processing for Myoelectric Control," in Powered Upper Limb Prostheses, A. Muzumdar, Ed. Berlin Heidelberg: Springer-Verlag, 2004, pp. 35-54.

[20] R. Reiter, "Eine Neue Electrokunsthand," Grenzgebite der Medizin, vol. 4, p. 133, 1948.

[21] R. N. Scott and P. A. Parker, "Myoelectric prostheses: state of the art.," J. Med. Eng. Technol., vol. 12, no. 4, pp. 143-51, Jan. .

[22] T. A. Kuiken, G. A. Dumanian, R. D. Lipschutz, L. A. Miller, and K. A. Stubblefield, "The use of targeted muscle reinnervation for improved myoelectric prosthesis control in a bilateral shoulder disarticulation amputee.," Prosthet. Orthot. Int., vol. 28, no. 3, pp. 245-53, Dec. 2004.

[23] T. A. Kuiken, G. Li, B. A. Lock, R. D. Lipschutz, L. A. Miller, K. A. Stubblefield, and K. B. Englehart, "Targeted muscle reinnervation for real-time myoelectric control of multifunction artificial arms.," JAMA, vol. 301, no. 6, pp. 619-28, Feb. 2009.

[24] G. N. Saridis and T. P. Gootee, "EMG pattern analysis and classification for a prosthetic arm.," IEEE Trans. Biomed. Eng., vol. 29, no. 6, pp. 403-12, Jun. 1982.

[25] B. Hudgins, P. Parker, and R. N. Scott, "A new strategy for multifunction myoelectric control.," IEEE Trans. Biomed. Eng., vol. 40, no. 1, pp. 82-94, Jan. 1993. 
[26] J. L. G. Nielsen, S. Holmgaard, N. Jiang, K. B. Englehart, D. Farina, and P. A. Parker, "Simultaneous and proportional force estimation for multifunction myoelectric prostheses using mirrored bilateral training.," IEEE Trans. Biomed. Eng., vol. 58, no. 3, pp. 681-688, Mar. 2011.

[27] A. B. Ajiboye and R. F. ff Weir, "A heuristic fuzzy logic approach to EMG pattern recognition for multifunctional prosthesis control.," IEEE Trans. Neural Syst. Rehabil. Eng., vol. 13, no. 3, pp. 280-91, Sep. 2005.

[28] E. Scheme and K. Englehart, "Electromyogram pattern recognition for control of powered upper-limb prostheses: state of the art and challenges for clinical use.," J. Rehabil. Res. Dev., vol. 48, no. 6, pp. 643-59, Jan. 2011.

[29] C. Cipriani, C. Antfolk, M. Controzzi, G. Lundborg, B. Rosen, M. C. Carrozza, and F. Sebelius, "Online myoelectric control of a dexterous hand prosthesis by transradial amputees.," IEEE Trans. Neural Syst. Rehabil. Eng., vol. 19, no. 3, pp. 260-70, Jun. 2011.

[30] S. Amsuess, P. Gobel, B. Graimann, and D. Farina, "A Multi-Class Proportional Myocontrol Algorithm for Upper Limb Prosthesis Control: Validation in RealLife Scenarios on Amputees.," IEEE Trans. Neural Syst. Rehabil. Eng., vol. 23, no. 5, pp. 827-836, Oct. 2014.

[31] N. Jiang, H. Rehbaum, I. Vujaklija, B. Graimann, and D. Farina, "Intuitive, online, simultaneous, and proportional myoelectric control over two degrees-offreedom in upper limb amputees.," IEEE Trans. Neural Syst. Rehabil. Eng., vol. 22, no. 3, pp. 501-10, May 2014.

[32] J. Mizrahi, Advances in Applied Electromyography. InTech, 2011.

[33] “COAPT LLC, Coapt." [Online]. Available: http://www.coaptengineering.com/. [Accessed: 09-Feb-2015].

[34] Vincent Systems, "VINCENTevolution 2." [Online]. Available: http://vincentsystems.de/en/prosthetics/vincent-evolution-2/. [Accessed: 20-May2015].

[35] C. Antfolk, M. D’Alonzo, B. Rosén, G. Lundborg, F. Sebelius, and C. Cipriani, "Sensory feedback in upper limb prosthetics.," Expert Rev. Med. Devices, vol. 10, no. 1, pp. 45-54, 2013.

[36] K. Keehoon, J. E. Colgate, J. J. Santos-Munne, A. Makhlin, and M. A. Peshkin, "On the Design of Miniature Haptic Devices for Upper Extremity Prosthetics," IEEE/ASME Trans. Mechatronics, vol. 15, no. 1, pp. 27-39, Feb. 2010.

[37] R. S. Armiger, F. V. Tenore, K. D. Katyal, M. S. Johannes, A. Makhlin, M. L. Natter, J. E. Colgate, S. J. Bensmaia, and R. J. Vogelstein, "Enabling closed-loop control of the Modular Prosthetic Limb through haptic feedback," Johns Hopkins APL Tech. Dig. (Applied Phys. Lab., vol. 31, no. 4, pp. 345-353, 2013.

[38] G. M. Goodwin, D. I. McCloskey, and P. B. Matthews, "The contribution of muscle afferents to kinaesthesia shown by vibration induced illusions of movement and by the effects of paralysing joint afferents.," Brain, vol. 95, no. 4, pp. 705-48, Jan. 1972.

[39] J. P. Roll and J. C. Gilhodes, "Proprioceptive sensory codes mediating movement trajectory perception: human hand vibration-induced drawing illusions.," Can. J. Physiol. Pharmacol., vol. 73, no. 2, pp. 295-304, Feb. 1995. 
[40] J. Gonzelman, H. Ellis, and O. Clayton, "Prosthetic device sensory attachment," US2656545 A, 27-Oct-1953.

[41] C. Cipriani, M. D’Alonzo, and M. C. Carrozza, "A miniature vibrotactile sensory substitution device for multifingered hand prosthetics.," IEEE Trans. Biomed. Eng., vol. 59, no. 2, pp. 400-8, Feb. 2012.

[42] "Engineering Acoustics, Inc., C2 Tactor." [Online]. Available: http://www.atactech.com/PR_tactors.html. [Accessed: 14-Nov-2015].

[43] K. O. Johnson, "The roles and functions of cutaneous mechanoreceptors," Curr. Opin. Neurobiol., vol. 11, no. 4, pp. 455-461, 2001.

[44] A. WILSKA, "On the vibrational sensitivity in different regions of the body surface.," Acta Physiol. Scand., vol. 31, no. 2-3, pp. 284-9, Jul. 1954.

[45] A. Ninu, S. Dosen, S. Muceli, F. Rattay, H. Dietl, and D. Farina, "Closed-loop control of grasping with a myoelectric hand prosthesis: which are the relevant feedback variables for force control?," IEEE Trans. Neural Syst. Rehabil. Eng., vol. 22, no. 5, pp. 1041-52, Sep. 2014.

[46] A. Chatterjee, P. Chaubey, J. Martin, and N. Thakor, "Testing a Prosthetic Haptic Feedback Simulator With an Interactive Force Matching Task," J. Prosthet. Orthot., vol. 20, no. 2, pp. 27-34, 2008.

[47] C. Pylatiuk, A. Kargov, and S. Schulz, "Design and evaluation of a low-cost force feedback system for myoelectric prosthetic hands," J. Prosthet. Orthot., vol. 18, no. 2, pp. 57-61, 2006.

[48] E. Banziger, "Wrist Rotation Activation in Myoelectric Prosthetics -- An Innovative Approach: A Case Study," ACPOC News, vol. 30, pp. 12-14, 1996.

[49] R. Butikofer and P. D. Lawrence, "Electrocutaneous Nerve Stimulation-I: Model and Experiment," IEEE Trans. Biomed. Eng., vol. BME-25, no. 6, pp. 526-531, Nov. 1978.

[50] G. B. Rollman, "Electrocutaneous stimulation.," in Conference on cutaneous communication systems and devices, 1974.

[51] A. Y. Szeto and F. A. Saunders, "Electrocutaneous stimulation for sensory communication in rehabilitation engineering.," IEEE Trans. Biomed. Eng., vol. 29, no. 4, pp. 300-8, Apr. 1982.

[52] H. Kajimoto, N. Kawakami, T. Maeda, and S. Tachi, "Tactile Feeling Display using Functional Electrical,’ Icat'99, no. August 2015, p. 8, 1999.

[53] G. F. Shannon, "A comparison of alternative means of providing sensory feedback on upper limb prostheses," Med. Biol. Eng., vol. 14, no. 3, pp. 289-294, May 1976.

[54] R. N. Scott, R. H. Brittain, R. R. Caldwell, A. B. Cameron, and V. A. Dunfield, "Sensory-feedback system compatible with myoelectric control.," Med. Biol. Eng. Comput., vol. 18, no. 1, pp. 65-9, Jan. 1980.

[55] Y. Sasaki, Y. Nakayama, and M. Yoshida, "Sensory feedback system using interferential current for EMG prosthetic hand," in Proceedings of the Second Joint 24th Annual Conference and the Annual Fall Meeting of the Biomedical Engineering Society] [Engineering in Medicine and Biology, vol. 3, pp. 24022403. 
[56] C. Hartmann, J. Linde, S. Dosen, D. Farina, L. Seminara, L. Pinna, M. Valle, and M. Capurro, "Towards prosthetic systems providing comprehensive tactile feedback for utility and embodiment," in 2014 IEEE Biomedical Circuits and Systems Conference (BioCAS) Proceedings, 2014, pp. 620-623.

[57] N. Jorgovanovic, S. Dosen, D. J. Djozic, G. Krajoski, and D. Farina, "Virtual grasping: Closed-loop force control using electrotactile feedback," Comput. Math. Methods Med., vol. 2014, 2014.

[58] G. F. Shannon, "Sensory feedback for artificial limbs.," Med. Prog. Technol., vol. 6, no. 2, pp. 73-9, Jan. 1979.

[59] J. Ning, S. Dosen, K.-R. Muller, and D. Farina, "Myoelectric Control of Artificial Limbs-Is There a Need to Change Focus? [In the Spotlight]," IEEE Signal Process. Mag., vol. 29, no. 5, pp. 152-150, Sep. 2012.

[60] T. W. Wright, A. D. Hagen, and M. B. Wood, "Prosthetic usage in major upper extremity amputations.," J. Hand Surg. Am., vol. 20, no. 4, pp. 619-22, Jul. 1995.

[61] S. Lewis, M. F. Russold, H. Dietl, and E. Kaniusas, "Satisfaction of Prosthesis Users with Electrical Hand Prostheses and their Sugggested Improvements.," Biomed. Tech. (Berl)., Sep. 2013.

[62] M. K. Glynn, H. R. Galway, G. Hunter, and W. F. Sauter, "Management of the upper-limb-deficient child with a powered prosthetic device.," Clin. Orthop. Relat. Res., no. 209, pp. 202-5, Aug. 1986.

"DARPA." [Online]. Available: http://www.darpa.mil/Our_Work/BTO/Programs/Revolutionizing_Prosthetics.as px. [Accessed: 09-Feb-2015].

[64] "RSL Steeper, Inc. Bebionic prosthetic hand." [Online]. Available: http://bebionic.com/. [Accessed: 12-Apr-2015].

[65] "Touch Bionics, Inc. i-Limb prosthetic hand." [Online]. Available: http://www.touchbionics.com/. [Accessed: 12-Apr-2015].

[66] S. Amsuess, P. Goebel, B. Graimann, and D. Farina, "Extending mode switching to multiple degrees of freedom in hand prosthesis control is not efficient.," Conf. Proc. Annu. Int. Conf. IEEE Eng. Med. Biol. Soc., vol. 2014, pp. 658-61, Aug. 2014.

[67] B. A. Lock, Design and Interactive Assessment of Continuous Multifunction Myoelectric Control Systems. 2005.

[68] B. Peerdeman, D. Boere, H. Witteveen, R. Huis in 'tVeld, H. Hermens, S. Stramigioli, H. Rietman, P. Veltink, S. Misra, R. H. in 't Veld, H. Hermens, S. Stramigioli, H. Rietman, P. Veltink, and S. Misra, "Myoelectric forearm prostheses: State of the art from a user-centered perspective," J. Rehabil. Res. Dev., vol. 48, no. 6, pp. 719-37, Jan. 2011.

[69] S. Dosen, M. Markovic, N. Wille, M. Henkel, M. Koppe, A. Ninu, C. Frömmel, and D. Farina, "Building an internal model of a myoelectric prosthesis via closed-loop control for consistent and routine grasping.," Exp. brain Res., vol. 233, no. 6, pp. 1855-65, Jun. 2015.

[70] R. E. Johnson, K. P. Kording, L. J. Hargrove, and J. W. Sensinger, "Does EMG control lead to distinct motor adaptation?," Front. Neurosci., vol. 8, p. 302, Jan. 
2014.

[71] M. Markovic, S. Dosen, C. Cipriani, D. Popovic, and D. Farina, "Stereovision and augmented reality for closed-loop control of grasping in hand prostheses.," $J$. Neural Eng., vol. 11, no. 4, p. 046001, Aug. 2014.

[72] M. Markovic, S. Dosen, D. Popovic, B. Graimann, and D. Farina, "Sensor fusion and computer vision for context-aware control of a multi degree-of-freedom prosthesis.," J. Neural Eng., vol. 12, no. 6, p. 066022, Nov. 2015.

[73] S. Dosen, M. Markovic, C. Hartmann, and D. Farina, "Sensory feedback in prosthetics: a standardized test bench for closed-loop control.," IEEE Trans. Neural Syst. Rehabil. Eng., vol. 23, no. 2, pp. 267-76, Mar. 2015.

[74] S. Dosen, M. Markovic, S. Kelef, B. Graimann, and D. Farina, "EMG Biofeedback for online predictive control of grasping force in a myoelectric prosthesis," J. Neuroeng. Rehabil., vol. 12, no. 55, pp. 1-13, 2015.

[75] L. Bodenhagen, A. R. Fugl, A. Jordt, M. Willatzen, K. A. Andersen, M. M. Olsen, R. Koch, H. G. Petersen, and N. Kruger, "An Adaptable Robot Vision System Performing Manipulation Actions With Flexible Objects," IEEE Trans. Autom. Sci. Eng., vol. 11, no. 3, pp. 749-765, Jul. 2014.

[76] S. Chitta, E. Jones, M. Ciocarlie, and K. Hsiao, "Mobile Manipulation in Unstructured Environments: Perception, Planning, and Execution," IEEE Robot. Autom. Mag., vol. 19, no. 2, pp. 58-71, Jun. 2012.

[77] S. Dosen, M. Markovic, D. B. Popović, D. Farina, and B. Graimann, "Sensor Fusion for Control of Upper Limb Protheses: Integration of Myoelectric Control with Stereovision, Augmented Reality and Inertial Sensing," EP 13171671.4, 2013.

[78] "Creative Technology Ltd., Senz3D®." [Online]. Available: http://de.creative.com/p/web-cameras/creative-senz3d. [Accessed: 11-Feb-2015].

[79] "MathWorks, Inc., GPU Computing Support for NVIDIA CUDA-Enabled GPUs." [Online]. Available: http://www.mathworks.com/discovery/matlabgpu.html. [Accessed: 29-Jul-2015].

[80] M. A. Fischler and R. C. Bolles, "Random sample consensus: a paradigm for model fitting with applications to image analysis and automated cartography," Commun. ACM, vol. 24, no. 6, pp. 381-395, Jun. 1981.

[81] J. Canny, "A Computational Approach to Edge Detection," IEEE Trans. Pattern Anal. Mach. Intell., vol. PAMI-8, no. 6, pp. 679-698, Nov. 1986.

[82] M. R. Cutkosky, "On grasp choice, grasp models, and the design of hands for manufacturing tasks," IEEE Trans. Robot. Autom., vol. 5, no. 3, pp. 269-279, Jun. 1989.

[83] S. Došen and D. B. Popović, "Transradial prosthesis: artificial vision for control of prehension.," Artif. Organs, vol. 35, no. 1, pp. 37-48, Jan. 2011.

[84] Schweisfurth, Markovic, Dosen, Teich, and Farina, "Electrotactile EMG feedback improves the control of prosthesis grasping force, in review," J. Neural Eng., 2015.

[85] "Simulink Desktop Real-Time - MathWorks Deutschland." [Online]. Available: http://de.mathworks.com/products/simulink-desktop-real-time/. [Accessed: 02- 
Jun-2015].

[86] “Google Glass Project.” [Online]. Available: http://www.google.com/glass/start/.

[87] K. Østlie, I. M. Lesjø, R. J. Franklin, B. Garfelt, O. H. Skjeldal, and P. Magnus, "Prosthesis use in adult acquired major upper-limb amputees: patterns of wear, prosthetic skills and the actual use of prostheses in activities of daily life.," Disabil. Rehabil. Assist. Technol., vol. 7, no. 6, pp. 479-93, Nov. 2012.

[88] P. Parker, K. Englehart, and B. Hudgins, "Myoelectric signal processing for control of powered limb prostheses.," J. Electromyogr. Kinesiol., vol. 16, no. 6, pp. 541-8, Dec. 2006.

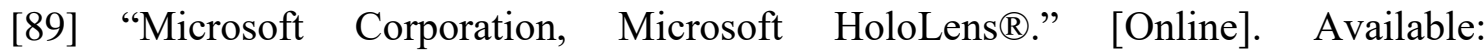
https://www.microsoft.com/microsoft-hololens/en-us. [Accessed: 15-Jun-2015].

[90] "Meta Inc., Meta 1 developer kit." [Online]. Available: https://www.getameta.com/. [Accessed: 15-Jun-2015].

[91] J. Aulinas, Y. Petillot, J. Salvi, and X. Lladó, "The SLAM problem: A survey," in Frontiers in Artificial Intelligence and Applications, 2008, vol. 184, no. 1, pp. 363-371. 


\section{APPENDICES}

Appendix 1: Stereovision and augmented reality for closed-loop control of grasping in hand prostheses 65

Appendix 2: Sensor fusion and computer vision for context-aware control of a multi degree-of-freedom prosthesis

Appendix 3: Sensory feedback in prosthetics: a standardized test bench for closed-loop control .69

Appendix 4: EMG Biofeedback for online predictive control of grasping force in a myoelectric prosthesis 
Sensor Fusion for Closed-loop Control of Upper-limb Prostheses 


\section{APPENDIX 1: STEREOVISION AND AUGMENTED REALITY FOR CLOSED-LOOP CONTROL OF GRASPING IN HAND PROSTHESES}


Sensor Fusion for Closed-loop Control of Upper-limb Prostheses 
Stereovision and augmented reality for closed-loop control of grasping in hand prostheses

This content has been downloaded from IOPscience. Please scroll down to see the full text.

2014 J. Neural Eng. 11046001

(http://iopscience.iop.org/1741-2552/11/4/046001)

View the table of contents for this issue, or go to the journal homepage for more

Download details:

IP Address: 134.76.10.66

This content was downloaded on 11/02/2016 at 21:03

Please note that terms and conditions apply. 


\title{
Stereovision and augmented reality for closed-loop control of grasping in hand prostheses
}

\author{
Marko Markovic ${ }^{1}$, Strahinja Dosen $^{1},{\text { Christian } \text { Cipriani }^{2} \text {, Dejan Popovic }}^{3}$ \\ and Dario Farina ${ }^{1,4}$ \\ ${ }^{1}$ Department of NeuroRehabilitation Engineering, Bernstein Focus Neurotechnology Göttingen, \\ Bernstein Center for Computational Neuroscience, University Medical Center Göttingen, Georg-August \\ University, D-37075 Göttingen, Germany \\ ${ }^{2}$ The BioRobotics Institute, Scuola Superiore Sant'Anna, V.le R Piaggio 34, I-56025 Pontedera, Italy \\ ${ }^{3}$ Faculty of Electrical Engineering, University of Belgrade, Bulevar kralja Aleksandra 73, \\ 11020 Belgrade, Serbia \\ E-mail: marko.markovic@bccn.uni-goettingen.de, strahinja.dosen@bccn.uni-goettingen.de, \\ ch.cipriani@sssup.it,dbp@etf.rs and dario.farina@bccn.uni-goettingen.de
}

Received 19 October 2013, revised 7 February 2014

Accepted for publication 4 March 2014

Published 3 June 2014

\begin{abstract}
Objective. Technologically advanced assistive devices are nowadays available to restore grasping, but effective and effortless control integrating both feed-forward (commands) and feedback (sensory information) is still missing. The goal of this work was to develop a user friendly interface for the semi-automatic and closed-loop control of grasping and to test its feasibility. Approach. We developed a controller based on stereovision to automatically select grasp type and size and augmented reality (AR) to provide artificial proprioceptive feedback. The system was experimentally tested in healthy subjects using a dexterous hand prosthesis to grasp a set of daily objects. The subjects wore AR glasses with an integrated stereo-camera pair, and triggered the system via a simple myoelectric interface. Main results. The results demonstrated that the subjects got easily acquainted with the semi-autonomous control. The stereovision grasp decoder successfully estimated the grasp type and size in realistic, cluttered environments. When allowed (forced) to correct the automatic system decisions, the subjects successfully utilized the AR feedback and achieved close to ideal system performance. Significance. The new method implements a high level, low effort control of complex functions in addition to the low level closed-loop control. The latter is achieved by providing rich visual feedback, which is integrated into the real life environment. The proposed system is an effective interface applicable with small alterations for many advanced prosthetic and orthotic/therapeutic rehabilitation devices.
\end{abstract}

Keywords: artificial proprioception, closed-loop control, control of grasping, stereovision, augmented reality

S Online supplementary data available from stacks.iop.org/JNE/11/046001/mmedia

(Some figures may appear in colour only in the online journal)

\footnotetext{
${ }^{4}$ Author to whom any correspondence should be addressed.
} 


\section{Introduction}

Reaching and grasping is a complex movement during which the human neuromusculoskeletal system accomplishes a number of very challenging tasks [1]. The process starts with the visual assessment of the target object to determine its location and orientation (required to correctly reach it) as well as its properties (shape and size required for prehension). This information is used by the brain to plan the motor task [2]. During the actual movement execution, the hand is transported to the target object and at the same time oriented and preshaped appropriately for the grasp based on the visual perception. The tactile and force feedback and proprioceptive feedback are used for online correction, especially during the contact phase. Importantly, these complex sensory-motor transformations are implemented by the neuromusculoskeletal system with remarkable ease and almost effortlessly for the subject, i.e., the subject is mostly concerned with the overall goal of the task (e.g., use of the object), while the actual implementation proceeds largely at a subconscious level (automatically). This natural control is made possible by a system of sophisticated sensory organs and human effectors in communication with the trained brain through a complex network of efferent and afferent channels, implementing the feed-forward and feedback sensorimotor schemes [3].

The grasping function, both motor and sensory aspects, can be lost or impaired as a result of a neurological injury, such as stroke, spinal cord injury, brachial plexus injury or limb amputation. Depending on the context, different assistive devices have been developed to restore grasping (e.g., functional electrical stimulation (FES) [4], exoskeletons [5] or prostheses [6]). Importantly, many of these devices are very advanced technological solutions striving to replicate the flexibility of the healthy human hand. Multichannel electrical stimulators with programmable timing and intensity can activate several muscles of the forearm in a coordinated fashion generating different grasps [4]. In recent years, several hand exoskeletons including separate kinematic chains for each finger have been developed and tested for force amplification and/or rehabilitation [7]. Similarly, modern prosthetic hands such as i-Limb from Touch Bionics [8] or Bebionic Hand from RSLSteeper [9] implement independent control of individual fingers. These robotic systems have enough mechanical flexibility to realize different grasp types, reflecting closely the repertoire used in daily life by a healthy human hand (e.g., palmar, lateral, pinch grip). However, user friendly control of grasping is still missing, and thereby the full capabilities of these advanced systems are largely unexploited in practice. Typically, only one grasp type is used [7] or the active grasp has to be selected manually through an unnatural (unintuitive) interface (e.g., pressing a button in FES [4], cocontracting antagonistic muscles in prosthetics [10]).

The control of grasping should include both a higher level interface for planning (grasp type, hand orientation and size selection) and a low level control of grasp execution, ideally providing also sensory feedback to the user (closedloop control). Effective and effortless implementation of these high and low level functions is a long standing challenge in rehabilitation engineering, with the most extensive research done in the field of prosthetics. In a typical approach, the control is realized by implementing a unidirectional communication pathway between the user and artificial system. The user generates all the necessary command signals (inputs), whereas the artificial controller captures the inputs, decodes them and operates the device accordingly. Therefore, the user is in control of the operation, and there is no automatic function that would decrease the need for large number of control inputs. Different signal sources have been tested to generate the control signals. In principle, high level motor commands for grasping can be detected directly at their origin (brain) using electroencephalography [11] or electrocorticography [12], but this approach is still far from being practical enough for use in daily life and requires complex instrumentation. In the previous studies, grasp type and size selection (high level control) were implemented by using foot movements detected by an insole equipped with foot switches [13], tongue control via a mouth piece integrating an inductive interface [14] and eye movements detected using electrooculography [15]. By far the most prevalent approach relied on the detection of the user's muscle activity $[10,16]$. Pattern recognition was applied to classify hand postures and even individual finger movements from the recorded multichannel surface electromyography (EMG) with high classification accuracies [17-19]. Also, implantable interfaces such as intra-fascicular neural electrodes [20] and implantable myoelectric sensors [21] have been tested for the same purpose. In contrast to a large body of work in recent years addressing the descending motor pathways, there are significantly fewer studies devoted to the restoration of feedback to the user. However, this was a popular research topic in the past [22, 23], and direct mechanical (e.g., vibrotactile or haptic $[24,25]$ ) or electrocutaneous stimulation [26] of the tactile sense were investigated as the means for providing the feedback. In some recent works, vibration motors [27], custom-made devices [28], electrical and hybrid stimulation [29] and also invasive approaches [30] have been tested.

Although success has been demonstrated, the establishment of the communication between the biological and artificial remained the challenge to be resolved. The main reason is that conventional man-machine interfaces used in practice have a limited bandwidth in both descending (feed-forward) and ascending (feedback) directions. Therefore, they cannot fully support the available functionality of the modern-day assistive devices. More recently, there are developments presented in the robotics literature inspired by human motor control demonstrating that a low-dimensional control input can be used to implement complex movements [31] and also facilitate learning [32]. A myoelectric controller presented in [33] implemented hand synergies to operate a dexterous hand prosthesis (three grasps) using only two myoelectric channels. These important advances should be used in the future to reduce the demands upon the user efforts regarding how to use the assistive system, and instead allow him/her to concentrate on the task to be accomplished.

We suggest here an approach for decreasing the burden on the user regarding how to use the assistive device. 
The idea is to enrich the artificial controller with extra sources of information, as also recently proposed in [34], in addition to the classically used human-generated command signals (e.g., myoelectric control), so that the controller is capable of autonomous decision making [35]. Consequently, certain system functions could be controlled fully automatically while the role of the user would be to supervise, correct and fine tune the system operation. The idea to automatize the assistive devices or facilitate the control using additional information sources has been addressed in the past. An early example is a prosthesis implementing automatic grasp selection based on the place of initial contact with the target object [36-38]. A commercially available prosthetic device, Sensor Hand Speed from Otto Bock [39], and some other recently presented systems [40, 41], integrate controllers that automatically compensate for object slipping. In [42] and [43], hierarchical control strategies in which myoelectric signals were used to trigger predefined high level functions (e.g., activating specific grasps) were developed and tested. Finally, an interesting sensor fusion approach, although for a different purpose, was presented in [44] where an eye tracker estimated the gaze direction, and this information was then combined with the myoelectric control to greatly improve reaching using a robotic arm.

We present a novel system for the semi-automatic and closed-loop control of grasping for assistive devices for the restoration of grasping function. As explained above, visual information is truly instrumental for the planning and execution of grasping. Therefore, we developed an artificial controller that employs state-of-the-art computer stereovision to emulate the human visual sense and automatically configure the hand into a preshape pattern that is appropriate for the target object. The fundamental components of the system are augmented reality (AR) glasses with embedded stereo cameras. The AR interface was employed to provide the user with augmented and intuitive visual feedback about the status of the hand for online correction of the grasp (i.e., closed-loop control). In particular, the AR interface is used to (virtually) project the hand into the field of view, thus informing the user about hand preshape even when it was out of view (artificial proprioception).

Automated grasp planning and execution using vision but also other interfaces such as Kinect have been addressed previously in the literature, especially in the context of autonomous robotics [45]. However, the system presented here is unique since it is designed for a specific context of human-machine interaction. Namely, it integrates highlevel functions of an automatic artificial controller with the volitional (biological) control of the user into a robust control loop operating online. The main aspect of this development is that the operational responsibility, and thus the cognitive load, are both shared between the system and the user.

The system as a whole, as well as some of the concepts and components, is being applied for the first time in this context (e.g., AR glasses, AR feedback, user-controller bidirectional communication, fusion of EMG and artificial vision). Therefore, the goal of this study was to present the control system and its components and then to evaluate the overall feasibility of the proposed approach. We tested the control system with healthy subjects who used a dexterous robotic hand in a simple manipulation task. Specifically, the goal was to address and evaluate: (1) the performance of the fully automatic as well as the semi-automatic control; (2) the user ability to successfully operate the system when sharing the control with the artificial controller; (3) the feasibility of the AR feedback by assessing if the user is able to properly perceive and successfully utilize the information provided by the AR interface. The results demonstrate that our approach is effective for the control of assistive devices, and the method becomes practical as the technologies that facilitate the method are developing rapidly and are convenient for daily use (e.g., Google glasses [46]).

\section{Material and methods}

\subsection{Overall control structure and system components}

Figure 1 summarizes the operation of the system and shows how the tasks and responsibilities are shared among different functional blocks. The user wears AR glasses with embedded stereo cameras. He/she triggers the operation of the semiautonomous controller via a simple myoelectric interface. The semi-autonomous controller selects grasp type and aperture size appropriate for the target object by processing the visual information retrieved by the stereo cameras. Accordingly, the controller sends a preshaping command to the hand. The selected grasp type and hand aperture size are visually fed back to the user through the AR glasses (AR objects). The user can employ the AR feedback to adjust the automatic decisions through the myoelectric interface. Eventually the controller implements hand closing to grasp and hand opening to release the object, in response to user myoelectric commands. The presented control is best described as semi-automatic since the user triggers the automatic operation but can also override/correct all of the decisions of the autonomous controller.

The system comprises the following components: (1) a standard laptop computer (8GB RAM, i5@2.73 GHz) equipped with a USB data acquisition card (DAQ 6210, National Instruments, US), (2) AR glasses (iWear920AR, Vuzix, UK), (3) a two-channel analogue myoelectric (EMG) amplifier (anEMG2, OTBioelettronica, IT) and (4) a robotic hand prosthesis prototype (IH2 Azzurra, Prensilia, IT).

The robotic hand was a left-handed, commercial version of the SmartHand [6]. It consists of four fingers and a thumb actuated by five electrical motors. Allowed motions are flexion/extension of the thumb, index, middle and ring-little finger as a couple and the rotation of the thumb opposition space. The hand includes encoders on each motor and an electronic controller that implements position control, by receiving commands sent over a serial bus from the laptop. The hand is mounted on a custom splint made from thermoplastic material and strapped firmly using Velcro straps and elastic bands to the subjects' forearm, so that the robotic hand is positioned directly beneath and parallel to the subjects' hand. Four different grasp types were implemented: palmar, lateral, bidigit pinch and tridigit pinch grasp. 


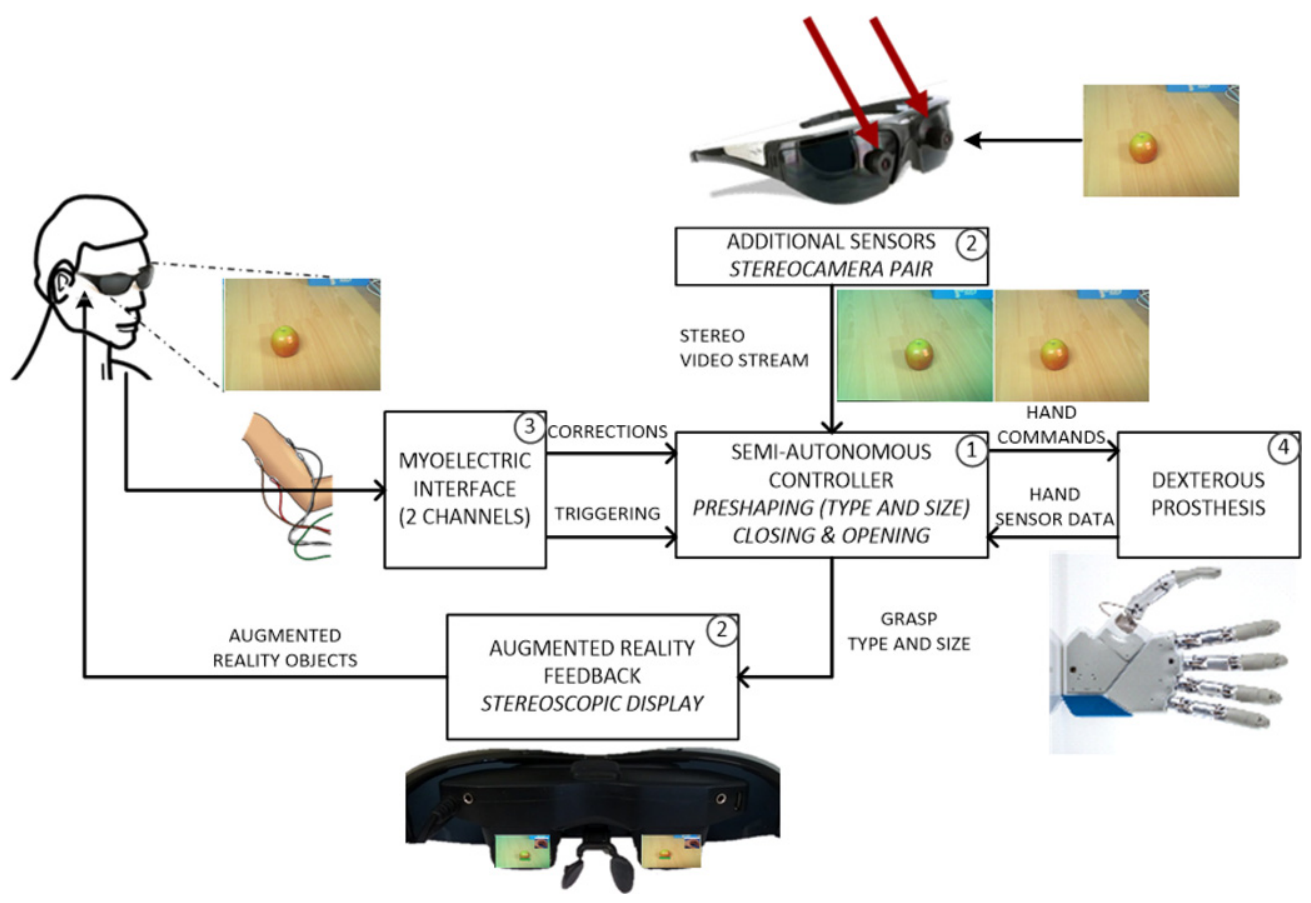

Figure 1. System architecture. The system comprises the following components: (1) semi-autonomous controller implemented on a standard laptop equipped with a data acquisition card, (2) AR glasses with an integrated pair of stereo cameras, (3) two-channel myoelectric interface and (4) a multi-grasp robotic hand.

Each of the two channels of the EMG amplifier includes a second-order band pass Butterworth filter $(10$ and $500 \mathrm{~Hz})$. The amplifier gain is adjusted individually for each subject to obtain good signal-to-noise ratios (visual inspection). Two pairs of standard $\mathrm{Ag} / \mathrm{AgCl} \mathrm{EMG}$ electrodes (Neuroline 720, AMBU, USA) are placed over the left-hand flexor and extensor muscles, with a reference electrode on the wrist. The amplifier outputs are acquired by a data acquisition card ( $1 \mathrm{kHz}$ sampling rate) connected to the laptop.

AR glasses are worn by the subjects. The glasses include stereo cameras embedded into the glass frames (resolution: $640 \times 480$ pixels, refresh rate: $30 \mathrm{~Hz}$ ). The cameras are therefore directed towards the scene the user is looking at. The video stream acquired from each camera is sent to the laptop via a USB port. The image frames are processed and then projected via a VGA port at the two panels embedded in the glass frames. The panels implement a stereoscopic display and therefore allow the user to see a single 3D image of the scene in front of him/her (i.e., as if he/she is looking without the glasses). The scene contains both real and virtual objects (AR feedback).

\subsection{Control flow and algorithm implementation}

The control flow is implemented as a finite state machine in which transitions between the states are triggered by a simple two-channel myocontrol interface. A high-level representation of the state machine is shown in figure 2(a) and illustrated using snapshots in figure 2(b) and a detailed algorithm is given in table A1 in the appendix.

The user could start operating the system by directing his/her sight and thus the glasses to the target object (figure 2(b), panel 1). After recognizing the target object, the system would acknowledge to the user the recognition of the target in the form of an overlay covering the object (object selection AR feedback) (figure 2(b), panel 2) and the user could then issue the command for opening the hand. This action would trigger the semi-automatic control and the system would analyze the scene (computer vision) to determine the geometrical model and properties (size and shape) of the target object. Based on this analysis, the system would estimate the grasp type and aperture size appropriate for grasping the object and send a preshape command to the hand (figure 2(b), panel 3). The component for stereovision analysis and subsequent grasp type and size selection based on the artificial vision is named stereovision grasp decoder. The AR feedback about the automatically selected grasp type and aperture size would then be presented to the user (figure 2(b), panel 4). The grasp-type AR feedback is presented as a visual icon, i.e., an image in the upper right corner of the subject's field of view. The aperture-size AR feedback is presented in the form of a virtual box placed just next to the target object, with one side parallel to the support plane and the other side aligned with the front side of the object. The size of this AR box is proportional to the current hand aperture size as read from the hand sensors. The user could therefore assess whether the hand was appropriately sized to grasp the target object by simply comparing the size of the virtual box to the size of the actual target object. The user could exploit this feedback to adjust the aperture (e.g., aperture too small/large) or to restart the decision process from the beginning (e.g., wrong grasp type) by issuing specific EMG commands. If the user judged the grasp type and aperture as correct, then he/she could bring 
(a) SYSTEM STATE MACHINE

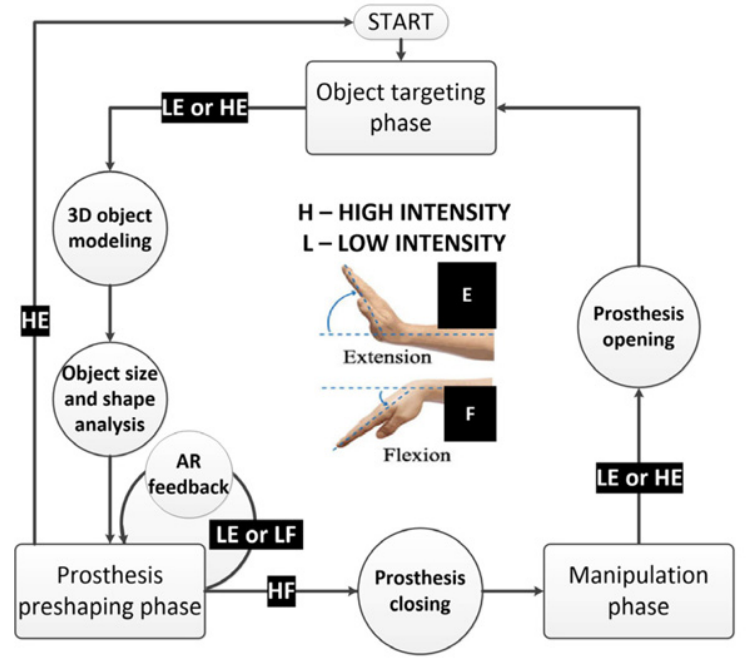

(b) CONTROL CYCLE SNAPSHOTS

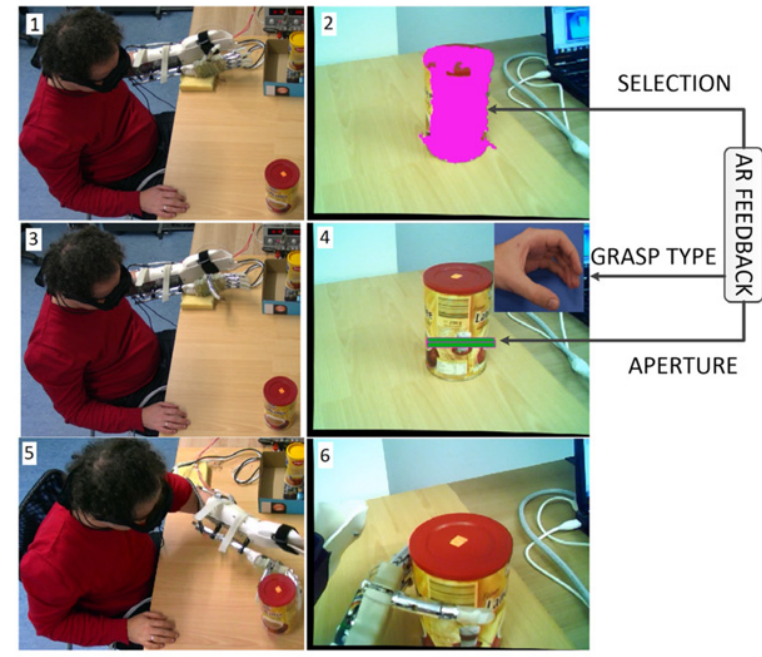

Figure 2. The control workflow: (a) system state machine and (b) control cycle in snapshots (left panel shows the setup and right panel depicts what the user actually saw through the glasses). The control loop operated as a state machine with the state transitions triggered by a simple two-channel myocontrol interface (high/low $\times$ flexion/extension). From top to bottom, the snapshots depict: (1) object targeting phase with AR feedback about object selection, (2) hand preshaping phase with AR feedback on the selected grasp type and aperture size and (3) hand closing phase.

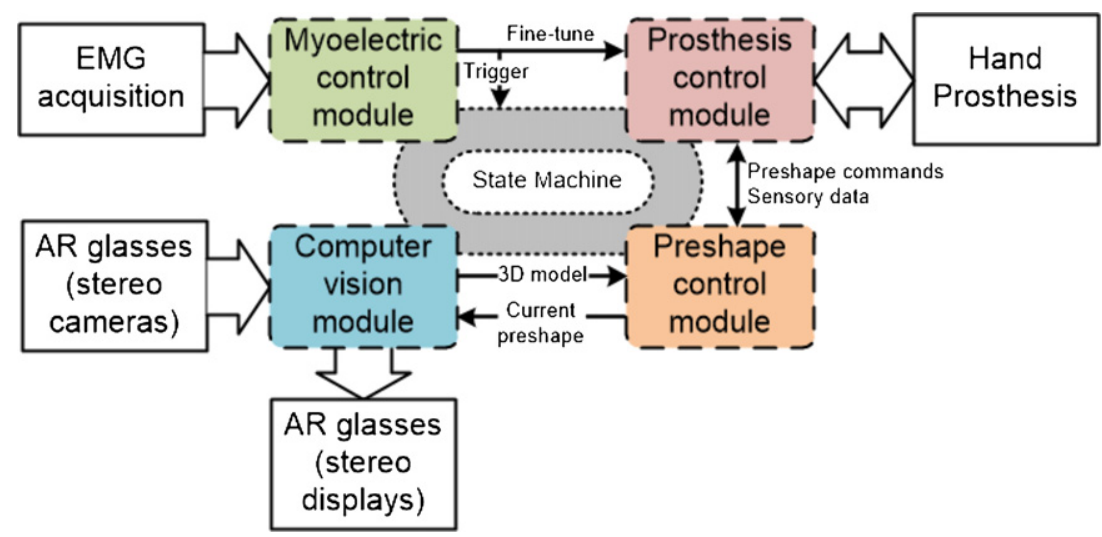

Figure 3. The interconnection of the system control software components and data flow between them. The online control software was implemented in Matlab and organized in the form of several modules with well-defined inputs and outputs.

the hand into the object vicinity and issue the EMG command to close it (figure 2(b), panels 5 and 6), manipulate the object as wished and finally reopen the hand in order to release the object (and restart). The described control system was running online and the average time needed to respond to a preshape command was less than $1 \mathrm{~s}$ (using the equipment described in the previous paragraph). This time includes command detection, stereovision processing and sending of the preshape commands to the hand.The online control software running on the laptop was implemented in Matlab 2012 and organized as a set of individual modules shown in figure 3 and described in the following. For more detailed information, see the supplementary data document attached to this paper (available from stacks.iop.org/JNE/11/046001/mmedia).

Myoelectric control module. This module implements the online acquisition and processing of the EMG signals.
The inputs for the processing are the EMG signals pickedup from the flexor and extensor muscles of the hand. The output is a 4-bit code indicating four possible user-generated commands (flexion/extension $\times$ high/low). Four commands are generated by using two-level thresholding of the RMS calculated over a $200 \mathrm{~ms}$ window (with $50 \%$ overlap) of raw EMG, and 'the first signal wins' strategy was used to resolve the eventual co-activation. This 4-bit code is fed into the state machine (figure 3 ) to trigger the state transitions and into the prosthesis control module to adjust the grasp properties (type and aperture size) (figure 3). Figure 4 demonstrates how myoelectric processing was used to command the system throughout the whole operation sequence (figure 2).

Computer vision module. This module is responsible for the bidirectional communication with the AR glasses, i.e., acquisition of the images from the stereo cameras 


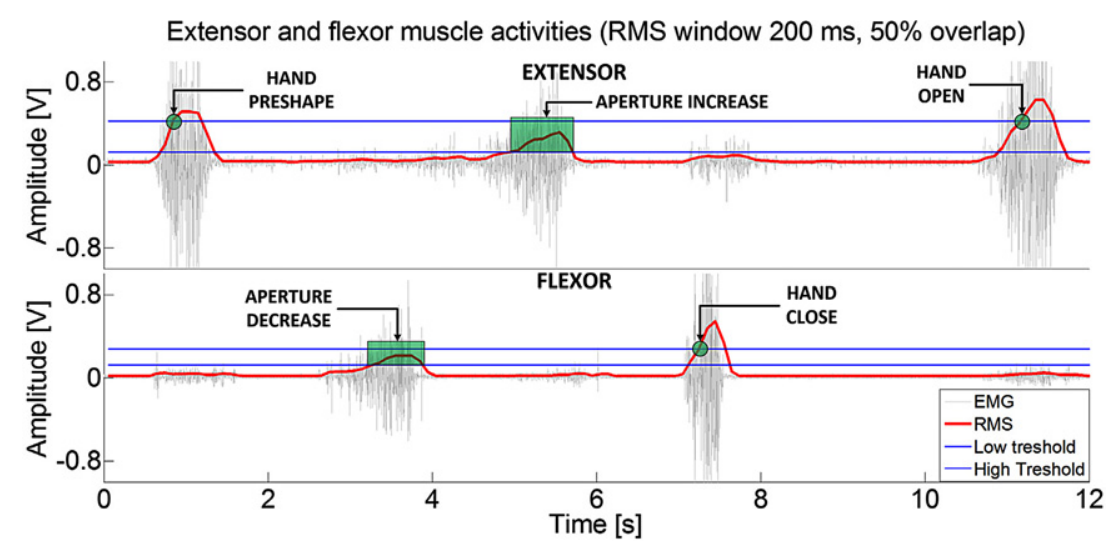

Figure 4. An example of myoelectric control during a complete trial. Top and bottom plots show the extensor and flexor activity, respectively. The thresholds for low and high activation are depicted using horizontal lines. Note that the thresholds are different for the two channels. Initially, the subject generated a high-level extensor contraction to open the hand. In response, the system initiated computer vision processing and automatically preshaped the hand. The subject then fine-tuned the hand aperture by generating low level contractions of the flexor (decrease aperture) and extensor (increase aperture). Finally, high flexor activation triggered the automatic hand closing, and thus the object was grasped. The trial ended with the automatic hand opening in response to a high level extensor contraction.

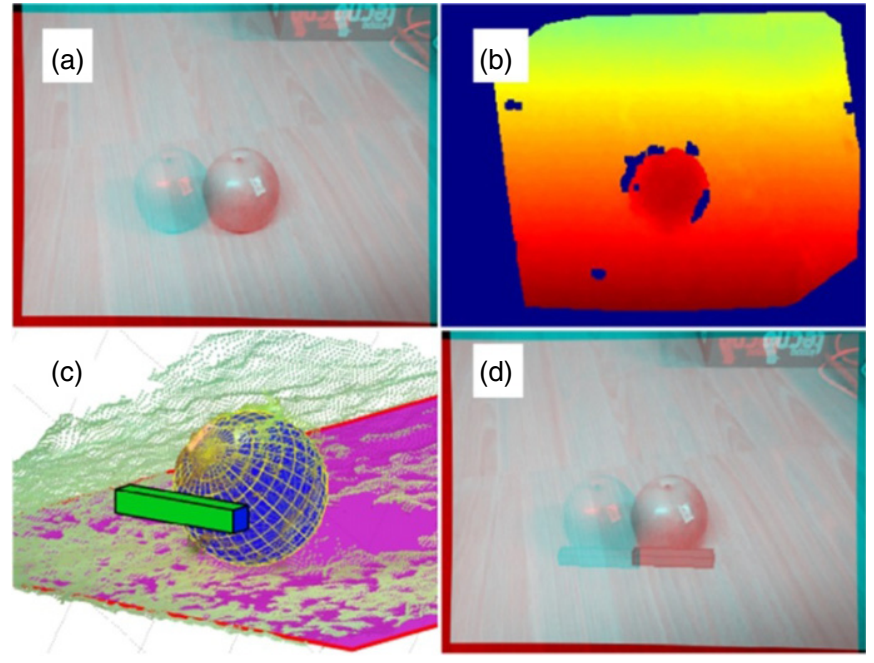

Figure 5. Illustration of the computer vision processing pipeline (computer vision module): (a) stereo pair showing the target object (an apple), (b) depth image (warmer colors denote the pixels closer to the viewer), (c) geometrical model (sphere) fitted through the cloud of points representing the target object and AR feedback object (green box) inserted into the 3D image, and (d) AR object projected into the pair of stereo images that are sent to the stereoscopic display within the AR glasses.

and control of the stereoscopic displays, and also for the processing of the image data. The inputs for processing are the two images from the stereo camera pair, and the current preshape of the hand (grasp type and aperture size) supplied by the preshape control module. The first input is used to estimate the properties of the target object (control) and the second to construct the AR feedback object (sensory feedback). The module implements a state-of-the-art computer vision processing pipeline comprising the following steps (figure 5): (1) depth estimation (figures 5(a) and (b)), (2) object segmentation, (3) 3D point cloud generation, (4) fitting of a geometrical model (box, cylinder, sphere and line) through the point cloud (figure 5(c)), and (5) construction of the virtual objects (AR feedback) and their projection into the real scene (figures 5(c) and (d)). The outputs from the module are the target object properties (shape and size) and stereo images with the embedded AR feedback. The size of the object was given in centimeters. The object properties are input for the preshape control module, whereas the images are sent to the stereoscopic panels of the AR glasses to be presented to the user. Depth estimation is implemented using an efficient large-scale stereo matching (ELAS) algorithm, as described in [47], which is applied on the rectified grayscale image pair. The cloud point is computed from the disparities using triangulation in combination with the extrinsic and intrinsic properties of the camera system, which are identified during calibration [48]. The RANSAC algorithm [49] with application specific modifications is used for point cloud processing and object modeling.

Preshape control module. The inputs for this module are the object properties estimated by the computer vision module and the current state of the hand preshape provided by the prosthesis control module, whereas the outputs are the automatically selected grasp type and size. The grasp type was selected using cognitive-like processing represented by a set of IF-THEN rules similar to the ones described in [50] and [35], while the grasp size was a continuous variable calculated as the object size estimate plus heuristically adopted extra margin of $0.5 \mathrm{~cm}$. In essence, the rule base implemented a mapping from the object properties into an appropriate grasp type (i.e., IF OBJECT SHAPE is $X$ and OBJECT SIZE larger/smaller than $Y$ THEN use GRASP TYPE $Z$ ). Palmar grasp was used for wide cylindrical and spherical objects, lateral for thin box objects, tridigit and bidigit for smaller objects etc. The computer vision module together with the preshape control module constitutes the stereovision grasp decoder. 


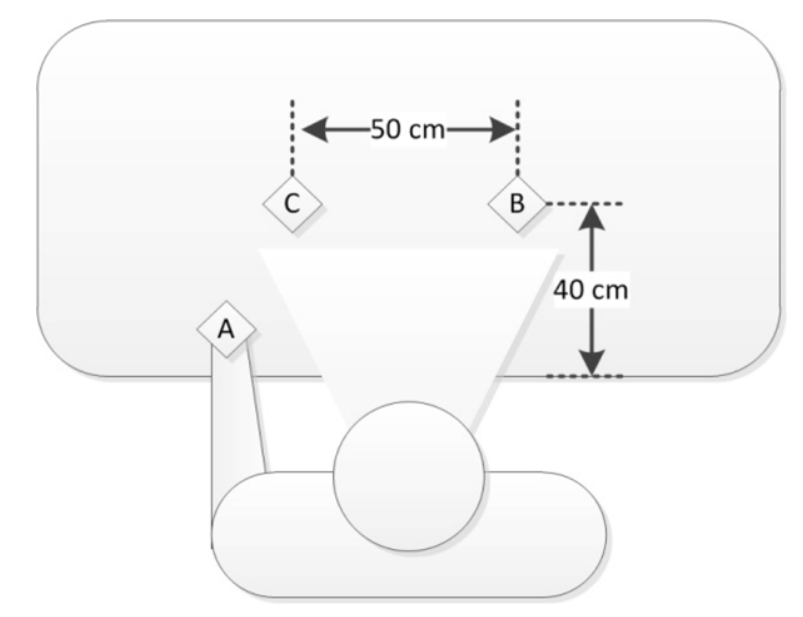

Figure 6. The experimental setup. The subjects were seated comfortably in front of a desk with three positions marked: (A) initial position to rest the prosthetic hand mounted on the subject's forearm by using a custom made splint, (B) initial position for the target objects and (C) final position to which the object had to be transported by the subject. The triangular area radiating from the subject towards the desk depicts the subject's field of view. Note that the hand was outside the subject's view when placed in the initial position.

Prosthesis control module. This module is responsible for the low level communication between the system and the hand prosthesis (i.e., sending commands and receiving sensor data over the serial bus with a sample rate of $20 \mathrm{~Hz}$ ). The inputs are desired grasp type and aperture size (automatic control) and the user commands for the correction of the hand aperture (manual control) and the output is the current preshape state determined by reading the prosthesis sensors. The prosthesis actuators are position driven. The position commands that should be sent to the hand in order to implement a grasp of certain type and size are specified using lookup tables. The latter are prepared beforehand by measuring the response of the hand to position commands covering the available range of motion.

\subsection{Experimental protocol}

The control system was tested by able-bodied subjects performing a simple grasping task. Thirteen healthy subjects $(29 \pm 4 \mathrm{yr})$ volunteered in the experiments after signing the informed consent form approved by the local ethics committee. The subjects were comfortably seated on an adjustable chair in front of a table where a workspace was organized (see figure 6). Three positions were marked on the workspace: the initial (rest) position for the hand, the initial position for the target object and a position to which the objects had to be transported after being grasped. The workspace was organized and the system was mounted so that the robot hand was outside the user's field of view while he/she was looking at the target object.

At the beginning of the session, the subject was given instructions about the system operation and experimental protocol and then he/she was allowed to briefly familiarize with the system (less than $10 \mathrm{~min}$ ). Twenty objects of different sizes and shapes (see table 1) were used as the targets and
Table 1. Tested objects, their sizes and corresponding grasp types.

\begin{tabular}{lll}
\hline Object & Size $(\mathrm{cm})$ & Grasp type \\
\hline Apple & 6.8 & Palmar \\
Coffee box & $10 \times 16.1$ & Palmar \\
Tea box & $6.5 \times 7.5 \times 16$ & Palmar \\
Mug & $8.2 \times 9.7$ & Palmar \\
Deodorant & $4.4 \times 17.2$ & Palmar \\
Tennis ball & 6.5 & Palmar \\
Thick pen & $1.8 \times 13.8$ & Lateral \\
Thin pen & $1 \times 14.3$ & Lateral \\
Fork & $1 \times 19.4$ & Lateral \\
Thin DVD box & $0.8 \times 12.5 \times 14$ & Lateral \\
Thick DVD box & $2.5 \times 14 \times 17.3$ & Lateral \\
Small plastic brick & $0.7 \times 2 \times 4$ & Bidigit pinch \\
Large bottle cap & $3.5 \times 2$ & Bidigit pinch \\
Small bottle cap & $2.6 \times 1$ & Bidigit pinch \\
Chestnut & $3 \times 1$ B & Bidigit pinch \\
USB stick & $1.3 \times 2.6 \times 6.1$ & Tridigit pinch \\
Big plastic brick & $3 \times 3 \times 4$ & Tridigit pinch \\
Espresso cup & $4.9 \times 5.3$ & Tridigit pinch \\
Lip crème & $1.9 \times 6.7$ & Tridigit pinch \\
Crème box & $1.8 \times 5$ & Tridigit pinch \\
\hline
\end{tabular}

were presented to the subjects in a randomized order. Subjects were asked to reach, grasp, transport and release each of the target objects by operating the artificial hand using the semiautonomous control loop explained in the previous paragraphs. The subjects were instructed to place the hand back in the initial position after the completion of each trial. Subjects operated the hand within four different experimental conditions with 5-10 min rest between the conditions.

(1) Automatic control with AR feedback (AUTO-AR). In this condition, the subjects could not correct the decisions made by the system/stereovision grasp decoder (refer to table A1 in the appendix). Two series of 20 trials were performed. This condition was used to assess the baseline performance when the control was fully automatic. The AR feedback did not play a useful role in this condition but was displayed to the subjects so they could familiarize with it.

(2) Semi-automatic control with AR feedback (SEMI-AR). In this condition, the system operated according to the full control sequence (without restrictions). Therefore, in addition to triggering the automatic control the user was able to correct the system decisions (grasp type and size) by relying on the AR feedback (refer to table A1 in the appendix). The subjects could decide if and how much to correct. They were instructed neither to look at the hand nor to move it from the initial position until the corrections were completed. This meant that all the adjustments had to be accomplished by relying exclusively on the AR feedback. The test included one series of 20 trials and the goal was to compare the performance of semi-automatic control with respect to the fully automatic control (AUTO-AR).

(3) Semi-automatic control with AR feedback and random errors (SEMI-AR-RE). The control was the same as in the 
Table 2. Outcome measures.

\begin{tabular}{|c|c|c|}
\hline Class & Name & Description \\
\hline $\mathrm{GC} 1^{\mathrm{a}}$ & Size estimation error & $\begin{array}{l}\text { Absolute difference between the actual size }(D) \\
\text { and estimated object size } d \text { : } \mathrm{GC} 1=|d-D|\end{array}$ \\
\hline GC2 & Hand aperture success rate & $\begin{array}{l}\text { The per cent of trials in which the selected hand } \\
\text { aperture } s \text { was: } s>(D-0.5 \mathrm{~cm}) \text { (otherwise, } \\
\text { the hand was not open enough to grasp the object) }\end{array}$ \\
\hline GC3 & Grasp type success rate & $\begin{array}{l}\text { The per cent of trials in which the selected grasp } \\
\text { type matched the correct grasp type for the given } \\
\text { object as defined in table } 1 .\end{array}$ \\
\hline GC4 & Preshape success rate & $\begin{array}{l}\text { The per cent of trials in which both the grasp } \\
\text { type and aperture size were correct (as defined above). } \\
\text { In this case, the hand was perfectly preshaped } \\
\text { to grasp the target object. Thus, GC } 4 \text { was } \\
\text { considered as the primary measure of performance. }\end{array}$ \\
\hline $\mathrm{TA} 1^{\mathrm{b}}$ & Task accomplishment success rate & $\begin{array}{l}\text { The per cent of trials in which the task } \\
\text { was successfully completed (the user grasped } \\
\text { the object, lifted it off the table, } \\
\text { brought it to the final position and released it). }\end{array}$ \\
\hline TA2 & Grasp control task failure rate & $\begin{array}{l}\text { The per cent of trials in which the user } \\
\text { failed the task due to an incorrectly } \\
\text { selected grasp type or size. }\end{array}$ \\
\hline TTA & $\begin{array}{l}\text { Time needed for the task } \\
\text { accomplishment }\end{array}$ & $\begin{array}{l}\text { The time interval from the moment when the } \\
\text { first hand preshaping command was issued } \\
\text { till the moment when the first closing command occurred. }\end{array}$ \\
\hline
\end{tabular}

SEMI-AR condition but the computer vision module was programmed to introduce random errors $( \pm 2-5 \mathrm{~cm})$ in the estimated hand aperture size. This error was added only after the grasp type was determined. As a result the estimated grasp type was (mostly) correct but the hand aperture was surely wrong forcing the subject to readjust the hand based on the visual information provided by the AR feedback as in the SEMI-AR condition (i.e., without looking at the hand). Again, it was up to the subject to decide when and how much to correct. This scenario included two series of 20 trials and the goal was to test the general perception and usefulness of the AR feedback by forcing the subjects to rely on it while finely adjusting the aperture size in practically every trial.

(4) Semi-automatic control with random errors and without the AR feedback (SEMI-VIS-RE). The system operated identically as in the SEMI-AR-RE condition but with the AR feedback turned off. The subjects now had to bring the hand close to the object and correct the aperture by directly comparing the hand to the object. This condition was tested in one series of trials and was used as the control condition for the SEMI-AR-RE.

\subsection{Data analysis}

The correct automatic control of the prosthesis and the task accomplishment were correlated but not in an absolute sense. Indeed, the task could be accomplished although a wrong grasp and/or size were selected (e.g., lateral grasp to pick up a bottle or hand fully opened to grasp a small object). To take this into account, we adopted two sets of outcome measures (see table 2), the first to evaluate the performance of the automatic or semi-automatic control of grasping (GC1-4) and the second to assess the actual accomplishment of the task (TA1-2). Additionally, in order to further quantify the stereovision grasp decoder performance and compare different operating modes, we have also measured the time from the moment when the first hand preshaping command was issued till the moment when the first closing command occurred, which is in the further text referred to as the time needed for the task accomplishment (TTA, see figure 4). Note that $100 \%$ - (TA1 + TA2) represents a per cent of the cases in which both grasp type and size were correct but the user still failed to complete the task. Put differently, this outcome measure includes the trials in which the automatic or semi-automatic grasp control was successfully accomplished, but the subjects failed the task afterwards, due to some other reasons. This could be, for example, an unintentional myoelectric triggering while approaching the object or a slippage of the object from the hand due to the prosthesis construction. We grouped these cases together into a common category of myoelectric/prosthesis control failure. As explained above, although this measure does reflect the overall performance of the conglomerate system, it is not related to its core features, which are automatic and semi-automatic grasp control. In the case of the semiautomatic control (SEMI-AR, SEMI-AR-RE, SEMI-VIS-RE), the outcomes GC2-4 were measured after the subject had fine-tuned the system, whereas in the case of the automatic control (AUTO-AR), they were the values estimated by the stereovision grasp decoder. In the SEMI conditions, we also registered if the user corrected the controller decisions (aperture size, grasp type). We assumed that the user corrected 

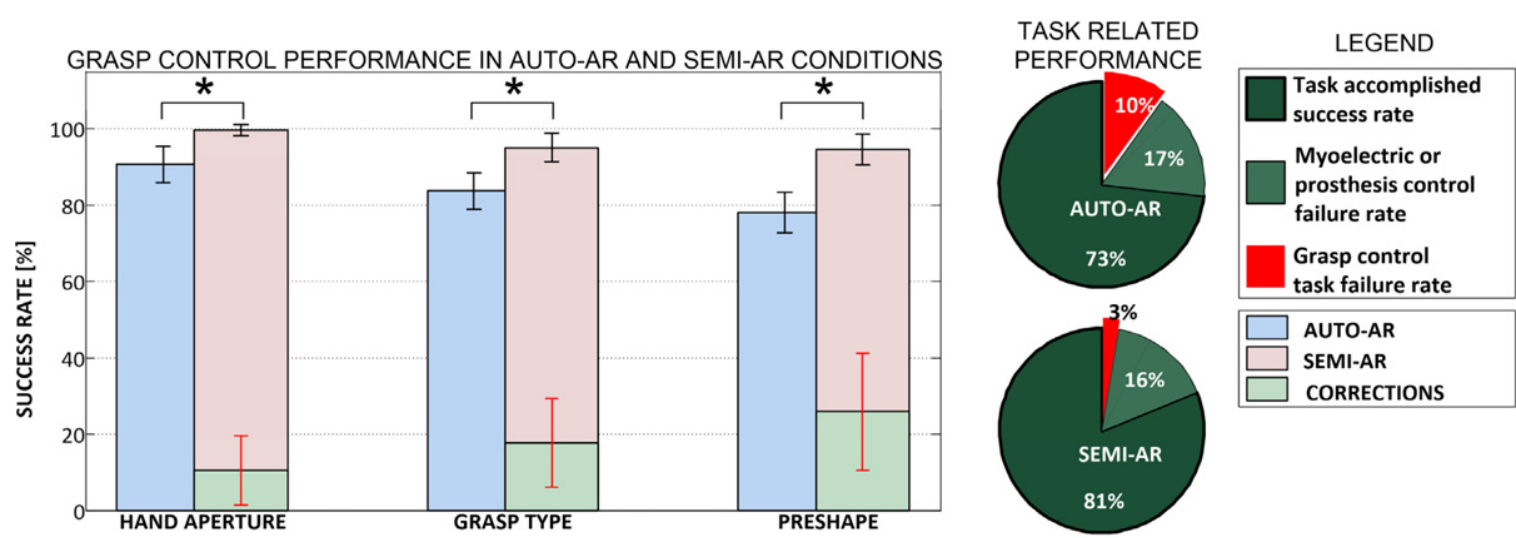

Figure 7. The grasp control and task related performance in the AUTO-AR and SEMI-AR experimental conditions. The overall rate of user corrections during the SEMI-AR is also depicted. The bars represent the mean and standard deviation of the grasp control outcome measures (GC2-4, table 2). The statistically significant differences are denoted by a star $(p<0.05)$. The pie charts depict the per cent rate for the task related performance indices (TA1-2, table 2). Note that the semi-automatic control resulted in significantly improved performance.

the preshape if he/she corrected either the grasp type or the aperture size. We used a two-sided $t$-test for dependent samples (repeated measures) for pair-wise comparison between the conditions. A $p$-value of 0.05 was selected as the threshold for statistical significance.

\section{Results}

Thirteen subjects performed a total of 1560 trials $(13$ subjects $\times 6$ series $\times 20$ objects $)$. Thus, the stereovision grasp decoder estimated a grasp type and an aperture size 1560 times. Overall, correct estimations of size and grasp type accounted for $91 \%$ and $90 \%$ of the cases, respectively. The preshape success rate (i.e., correct grasp type and aperture size) was $84 \%$. The average size estimation error was $0.73 \pm$ $1.08 \mathrm{~cm}$ (mean \pm standard deviation).

Figure 7 summarizes the results for the first (AUTO-AR) and second (SEMI-AR) experimental conditions which included $520(13$ subjects $\times 2$ series $\times 20$ objects $)$ and 260 trials $(13$ subjects $\times 1$ series $\times 20$ objects $)$, respectively. When the user was allowed to correct for the errors (SEMIAR), the performance improved significantly (bars in figure 7). The average improvement was $10 \%, 11 \%$ and $16 \%$ for GC2 (hand aperture success rate), GC3 (grasp type success rate) and GC4 (preshape success rate), respectively. The resulting performances were close to $100 \%$ : $99 \pm 5 \%, 94 \pm 9 \%$ and $94 \pm 5 \%$ for GC2, GC3 and GC4, respectively. The subjects intervened in $25 \%$ of the cases either by adjusting the hand aperture size or grasp type. A representative example of the correction of the hand aperture is shown in figure 8 . The task accomplishment outcome measures (TA1-2) are shown in the pie charts in figure 7. During the AUTO-AR condition the task was successfully completed in $73 \%$ of trials. Successful completion of the task increased significantly to $81 \%$ in the SEMI-AR condition $(p=0.041)$. This improvement was due to a reduction of the grasp control task failure rate (TA2 in table 2), which dropped from $11 \%$ to only $3 \%$. Therefore, when given the opportunity to correct and/or fine-tune the decisions of the decoder, the users were able to drive the prosthesis into

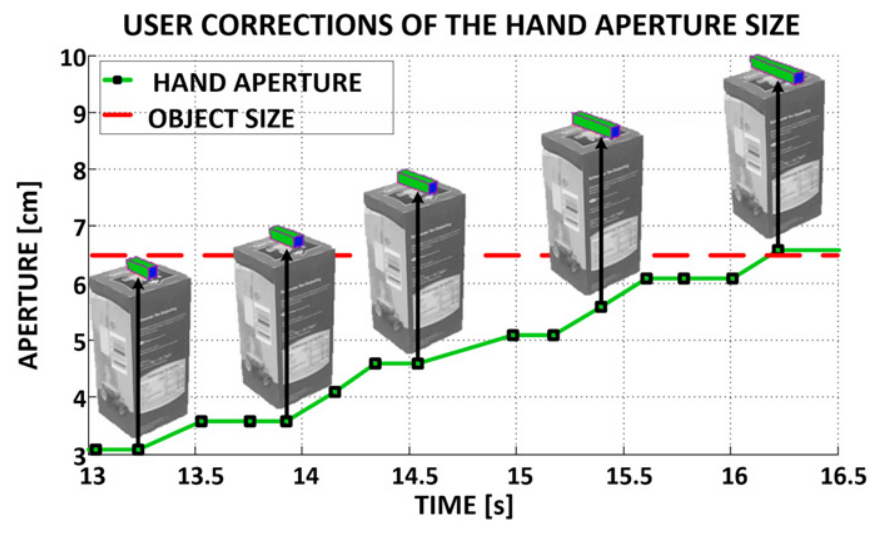

Figure 8. The sequence of user corrections of the hand aperture size. The subject relied on the AR feedback to implement the corrections while the hand was outside his/her field of view. The size of the AR box (green box) was initially smaller with respect to the size of the target object and the subject gradually opened the hand through a sequence of discrete steps (black points along the plot) until the two sizes approximately matched.

postures that yielded more stable grasps in the next phase, and thus were able to significantly improve the task completion rate (TA1). Furthermore, the average time the user spent for the task accomplishment (TTA) in the AUTO-AR scenario was $2.77 \mathrm{~s}$ in contrast to $3.47 \mathrm{~s}$ in the SEMI-AR (with no statistically significant difference, $p=0.065$ ). Therefore, we can conclude that in the SEMI-AR mode the user achieved significantly better performance compared to the AUTOAR without significantly increasing the average time needed to accomplish the task. Thus, the semi-automatic control mode of the presented system is preferred, as it offers clear performance advantages, with respect to the fully automatic control.

The results for the SEMI-AR-RE and SEMI-VIS-RE experimental conditions are presented in figure 9. In these conditions, random errors were added to the estimated aperture size. Consequently, the rate of user corrections for grasp 


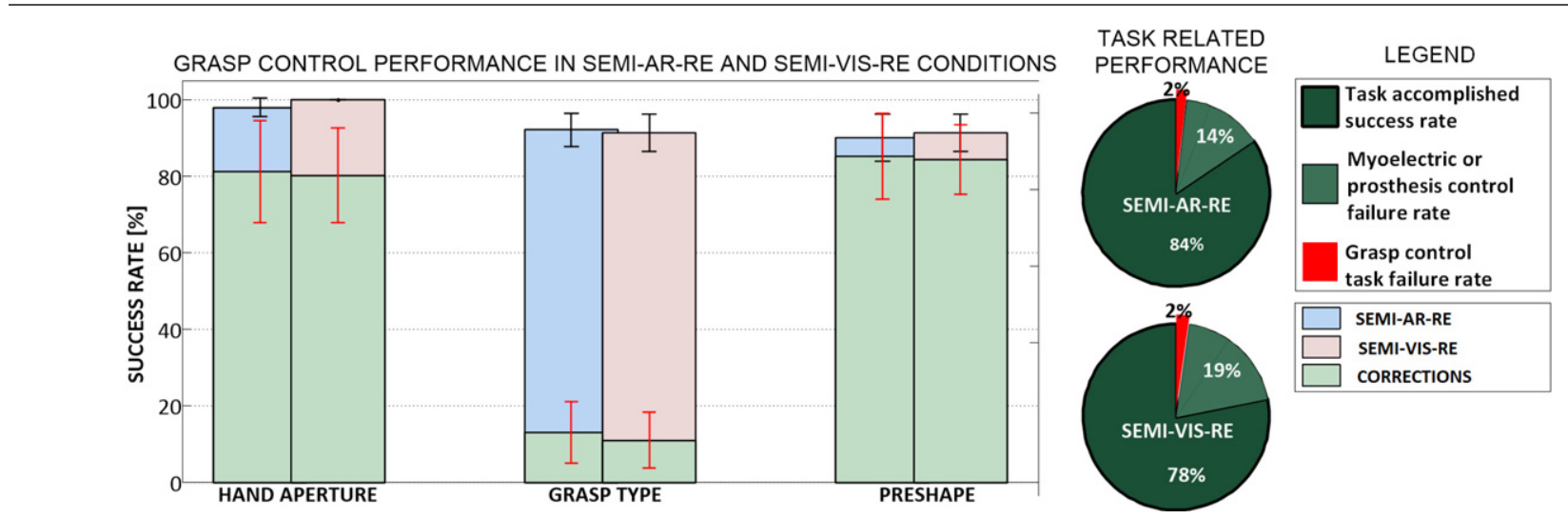

Figure 9. The grasp control and task related performance in the SEMI-AR-RE and SEMI-VIS-RE experimental conditions. The overall rate of user corrections during both scenarios is also depicted. The bars represent the mean and standard deviation of the grasp control outcome measures (GC2-4, table 2). The pie charts depict the per cent rate for the task related performance indices (TA1-2, table 2). Note that the performance measures and rate of user corrections were very similar in the two conditions.

size and the time needed to accomplish the task $(5.2 \mathrm{~s}$ in SEMI-AR-RE and $5.9 \mathrm{~s}$ in SEMI-VIS-RE) increased significantly compared to the SEMI-AR condition, whereas the rate of grasp type corrections did not statistically differ. The outcome measures achieved with SEMI-AR-RE (figure 9) did not statistically differ from those achieved with SEMI-AR (figure 7); only GC4 (preshape success rate) was statistically different $(p=0.041)$ and somewhat lower in the SEMIAR-RE condition (90\% versus 94\%). Therefore, although the subject was challenged by the poor performance of the automatic controller in SEMI-AR-RE, the overall performance was almost unaffected due to the possibility of manually adjusting the initial decisions of the stereovision grasp decoder. SEMI-AR-RE and SEMI-VIS-RE also resulted in a similar performance with no statistically significant differences $(p=$ $0.504, p=0.407, p=0.727, p=0.209$, and $p=0.977$ for GC2, GC3, GC4 and TA1, TA2 respectively). There was also no statistically significant difference in the time needed to accomplish the task (TTA) in these two conditions $(p=0.492)$. The fact that all performance measures were similar in both SEMI-VIS-RE and SEMI-AR-RE demonstrates that the subjects successfully utilized the AR feedback to correct the mistakes of the automatic controller. Therefore, the AR feedback is indeed a feasible medium to provide information to the user, i.e., in this specific case artificial-proprioception.

\section{Discussion}

As previously stated in introduction, the main contribution of this paper is a novel system that integrates volitional, biological and automatic, artificial control in order to decrease the burden on the user. This was achieved by fusing the information from two sources, i.e., artificial vision and myoelectric signals. In addition, the control loop was closed through the application of AR feedback, which is proposed as a method to further promote this integration by communicating to the user not only the prosthesis state but also internal information of the controller. The specific context (practical human-machine interaction) of the proposed method is directly reflected in the design decisions and selection of the system components. For example, in order to be robust and general, the vision module does not assume a predefined database of concrete objects. Also, the AR glasses are a wearable component which is likely to become even more ergonomic and convenient for the practical application due to the fast development of this technology (Google glasses [46]). We assessed the feasibility of this system by implementing a prototype that will be developed further in the future. The test was done in healthy subjects using an artificial hand prosthesis as a convenient experimental platform, but the proposed concept could be generalized to many assistive technologies for the restoration of reaching and grasping.

\subsection{Experimental evaluation}

The overall results for the performance of the stereovision grasp decoder (1560 trials in total) demonstrate that stereovision can be used to estimate the grasp relevant properties in a set of target objects with different shapes and sizes. The experimental task showed that after only a few minutes of training, the subjects were able to operate the system successfully and use the fully automatic control of grasping.

The autonomous operation was not flawless but the subjects were able to correct most of the errors by exploiting the AR feedback to fine-tune the decisions of the stereovision grasp decoder (semi-automatic control). The subjects successfully understood the meaning of the AR feedback, perceiving correctly the information about the grasp type and size. Also, subjects understood the bidirectional control loop, the concept of control sharing and they operated the system smoothly through different phases comprising the full control cycle (i.e., TTA was only few seconds). 
They successfully used the AR feedback information about the prosthesis state as well as controller decisions to make appropriate corrective actions when needed, bringing the control of grasping very close to an ideal performance $(\sim 100 \%)$. Thus, all of this demonstrates the feasibility of the overall conceptual solution and its components, such as stereovision grasp decoder and AR bidirectional communication interface. In addition, the rate of failure in the task accomplishment due to incorrect grasp control (TA2) was consistently very small $(\sim 2-3 \%)$ in all the SEMI-AR conditions. Importantly, the transition from automatic to semiautomatic control was almost effortless and consisted in the introduction of a few additional simple myoelectric commands to adjust the decisions of the stereovision grasp decoder. The closed-loop system demonstrated very good robustness when the user was challenged by the introduction of the intentional errors into the automatic control (SEMI-AR-RE). The errors were present in every trial and the error size was such that in most of the cases it would have been impossible to accomplish the grasp without a correction. However, this did not influence the overall performance.

Finally, the feasibility of the AR feedback was further confirmed by the last test (SEMI-AR-RE versus SEMI-VIS-RE) which demonstrated that the subjects were able to exploit the novel AR feedback as easily as the normal visual feedback. The subjects perceived the virtual object embedded into the real scene and were able to correctly compare its size against the size of the real objects as easily as when comparing the size of the real objects directly (hand opening versus object size). It is important to emphasize that the scenarios SEMI-AR-RE and SEMI-VIS$\mathrm{RE}$ do not necessarily reflect the expected use of the AR and normal visual feedback in the potential real application. It is likely that trained subjects will learn to rely more on the feed-forward control and use feedback only when necessary.

When analyzing the task completion success rate across the experimental scenarios it is notable that the behavior of the conglomerate system was also affected by failures unrelated to the automatic/semi-automatic control of grasping, but to the myoelectric control and prosthesis design (myoelectric/prosthesis control failure rate (figures 7 and 9). This depended on several factors: (1) individual ability of the subject to generate and control EMG signals as well as dexterity in prosthesis handling, (2) intrinsic properties of the prosthetic device and its mounting system and (3) a simple method to process the EMG signals. Therefore, these errors could be minimized by providing the prospective users with more training so that they can generate more consistent muscle activations while manipulating the prosthesis, by improving the prosthesis mechanical design (e.g., silicone coating over the fingers to prevent object slipping), and by implementing more robust methods for myoelectric control. These errors are general issues common to any prosthetic system and, as explained before, they do not reflect the performance of the core feature of the novel approach (semi-automatic control of grasping).

\subsection{Computer vision and automatic control of grasping}

Computer vision analysis was based on the standard state-ofthe-art methods. The performance in the automatic control of grasping achieved during the experimental sessions indicates that the computer vision module was relatively robust and reliable. The robustness is further illustrated in figure 10, which demonstrates the system operation in a rich, cluttered environment. The figure depicts several scenes in which the subject successfully selected and grasped four target objects. It is worth noting that in both cases the scene included many other objects in addition to the target. Nevertheless, the system correctly located and segmented the target object as shown by the semi-transparent green overlay representing the pixels allocated to the object (figure 10, left). The segmentation, modeling, size estimation and grasp type selection (figure 10, right) were all correct despite the richness of the scene and the fact that the target object was partially occluded by the object(s) placed in front of it.

The analysis of the scene by using stereovision is much more powerful and robust compared to the use of a single camera [35], but still suffers from well-known limitations like pixel correspondence problems [51]. This was also registered in our experiments. During the object targeting phase, the computer vision module sometimes failed to segment out the target object or there was a 'spillover' from the area of the target into the neighboring objects or the background. However, this was quite a rare event $(<3 \%)$ and it did not significantly influence the performance due to the following countermeasures: (1) the 'snapshot and analysis' was repeated continuously during targeting and an occasional failure would be most often corrected in the very next cycle; (2) the users could rely on the AR feedback to assess the quality of segmentation and avoid triggering when large errors were evident.

The system developed is an illustrative example of how an artificial controller can be enriched with an additional, nonconventional information source (stereo camera pair) and high level processing (cognitive like reasoning) to achieve fully automatic control of the functions that are conventionally the responsibility of the user (e.g., hand preshaping). In this scheme, the user is able to 'release' predefined 'motor programs' performing relatively complex functions, instead of continuously monitoring the task. This substantially simplifies the myoelectric interface, which only needs to implement a simple triggering mechanism, thereby reducing the burden on the user. This can be advantageous especially in the case of modern dexterous prosthetic hands and/or full arm prostheses. The presented control concept scales smoothly with the system complexity. In fact, the more complex the system is, the higher and more obvious will be a discrepancy between the necessary user efforts and required system functionality. For example, in the case of an entire upper limb prosthesis, the stereovision control could be used both to preshape the hand and to navigate the arm to reach and grasp the selected target object (stereovision servoing [52]). The complex 'preshape and reach program' could be triggered via a simple myoelectric command. 


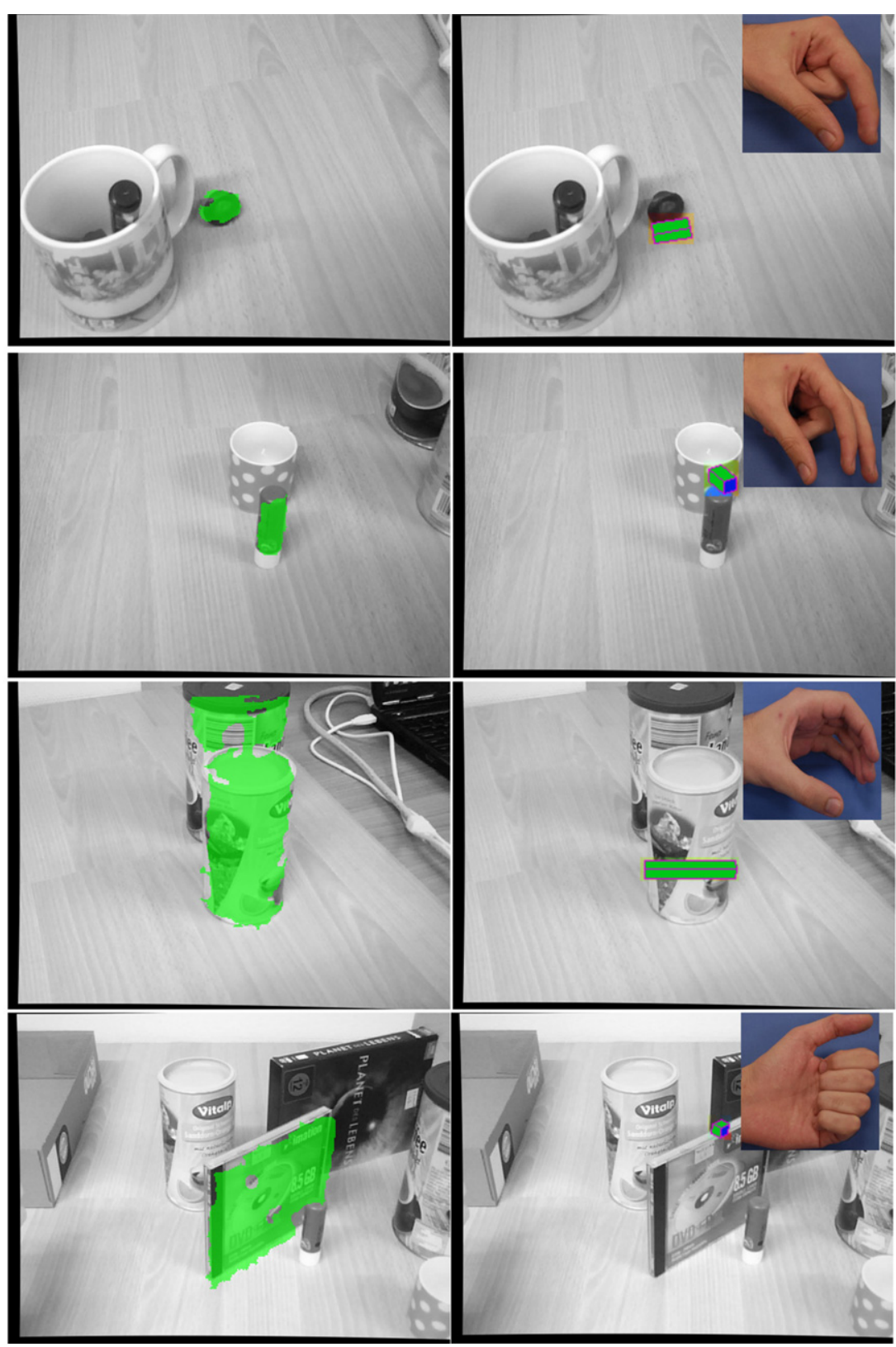

Figure 10. An example of system operation in a cluttered environment. The subject targeted several different objects (left side) placed within a realistic scene including several other colorful and textured objects and a background. The stereovision grasp decoder successfully segmented out the targets and selected an appropriate grasp type (grasp type icons) and size (the AR box with size similar to the target object) for each of them (right side).

\subsection{Augmented reality feedback and semi-automatic control}

The idea to close the loop 'through' the user by providing feedback about the state of the prosthesis is not novel [53]. However, the current work proposes a fundamentally novel interface to accomplish this goal. Compared to the 'classical' methods of electrical or direct mechanical stimulation, the most important advantage of the AR feedback is that it can exploit the high bandwidth and flexibility that is available within the visual communication channel. This is reflected both in the sheer volume of information that can be communicated to the subject as well as in the form in which that information can be presented. In the current work, the AR feedback was projected into the scene, next to the target object and a virtual box was used to implement 'artificial proprioception', i.e., the size of the box was proportional to the actual aperture size of the hand. As stated before, the system operation and experimental paradigm were not specifically designed to test the usefulness of the AR feedback and/or the potential advantages of the AR versus normal visual feedback. Instead, the aim was only to test the general feasibility since the AR interface has been used in this context for the first time, i.e., testing if the AR interface can be used as a component for implementing user corrections within a novel 
control system. However, even this first basic implementation demonstrates some possible benefits. Namely, as explained in the experimental protocol, in SEMI-AR and SEMI-AR-RE scenarios, the users operated the hand (i.e., correcting grasp type and size) without seeing it, i.e., the hand was outside the field of view and the subjects were looking at the object to be grasped. Note that this corresponds to the way grasping is performed in daily life by able-bodied subjects. Apart from promoting the normal use of the hand, artificial proprioception through AR can have some obvious advantages in certain situations, e.g., when the hand is partially occluded by some other object or is not completely visible from the user perspective, which is often the case during grasping (especially in cluttered environments). Moreover, the potential utility of AR feedback is even more evident when taking into account that the feedback could be much more sophisticated; for example, a full graphical model of the hand/prosthesis could be used to communicate complete and very detailed information in an intuitive way (e.g., position, contact and force of each individual finger). The flexibility and bandwidth of AR feedback were exploited in some other fields (e.g., robot-assisted surgeries [54]), but not in prosthetics which is a context with unique requirements. How to best utilize the potential of AR feedback in daily life prosthetic or clinical use is a very interesting and relevant question that will be addressed in the studies to follow.

To minimize the interference of AR feedback with the ongoing visual or other cognitive tasks, AR feedback could be simplified (e.g., a 2D object) and moved within the peripheral part of the visual field. The optimal form and positioning of AR feedback is an important question that will be addressed in future studies. It is also possible to combine several representations to best reconcile the needs of different tasks (e.g., unobtrusive, simple feedback most of the time and detailed feedback during specific, more demanding tasks).

A further novelty that was proposed in this work is the use of feedback not only to acknowledge to the user about the state of the device but also to allow him to monitor the decisions of the artificial controller. This gives the opportunity to the subject to supervise the automatic operation and take over control when needed, effectively implementing a bilateral communication between the user and controller. In the semi-automatic framework, the control is therefore shared between the two agents (user and controller) and the optimal integration of the two control loops (manual and automatic) will be an important question to address in future research.

\subsection{Perspectives and future work}

An important feature of the presented concept and corresponding implementation is a modular design. Strictly speaking, both stereovision and AR feedback could be used on their own, independently of each other. For example, the stereovision could implement automatic control, while the corrections are performed using conventional visual feedback (as in the SEMI-VIS-RE condition). Or, the AR could be used to provide feedback about the prosthetic device that is controlled 'manually' using a classic myoelectric control. The placement of the sensor element is also flexible. In the current implementation, the cameras were embedded within the front glasses, but a miniature sensor could be placed on the sides or above the glasses, for example, into the glass frame, or even incorporated into the hand or a prosthetic socket. Truly wearable and cosmetically acceptable, mobile solutions for the AR, such as specialized glasses and even contact lenses, are being developed and some models (e.g., Google Glass project [46]) are already available in limited quantities and are expected to become widely accessible very soon. This, together with the development and availability of fast processing cores within small form factor embedded systems, can provide the necessary technological framework for the practical implementation of the presented control concept. From the technical standpoint, the developed system relies on state-of-the-art technologies that are in the focus of current research efforts and are also fast developing. Although some of the components might not be ready for immediate practical application in daily life, this might change in the very near future. Stereovision is not the only method to implement the proposed control concept. The necessary information for the control of grasping is an estimate of the 3D shape of the target object and any technology providing a similar output (i.e., $3 \mathrm{D}$ point cloud) could be plugged into the control scheme in a straightforward manner. For example, the active methods based on IR depth sensing derived from Microsoft Kinect are more robust than passive stereovision. In general, this technology is developing very fast, evolving towards solutions that will soon become low cost and physically small, and thereby very convenient for practical applications (e.g., Prime Sense [55], Creative Senz3D [56]). Finally, advanced scene processing [57] and grasp planning methods [58] that were investigated intensively in robotics research could be used to improve the prosthesis preshape module, but the candidate solutions could be only those approaches that can cope with the constraints of this specific application (e.g., incomplete input information, responsiveness to user decisions, ad hoc scene etc).

Importantly, the presented approach and its components are a rather general solution that could be applied with relatively minor adjustments in many contexts. In principle, it could be used with any assistive system that includes a multi-grasp end effector (e.g., electrical stimulator, hand exoskeleton, hand prosthesis). To port the control scheme (figure 3) to a different device, the prosthesis control module would have to be replaced with a module providing a low level interface to that specific system. All the other components could remain unchanged or, in the worst case, they would have to be somewhat adapted to support the new application (e.g., available grasps). Alternatively, only a part of the functionality 
could be ported. For example, stereovision grasp decoder and myoelectric control could be used for grasp type and size selection in EMG-triggered FES. Similarly, AR glasses could be used not for control as in this study, but only to provide AR feedback, supplementing the impaired sensations in patients suffering from a neurological condition (e.g., stroke). As explained below, stereovision can be extended to the control of orientation, and in this case, the scope of potential applications is even larger: a rehabilitation robot for assistance in reaching, full arm prosthesis, a hybrid system combining a reaching robot and an FES device for grasping. Finally, the presented control system could indeed be used in daily life for prosthetic or orthotic applications, which is similar to the scenario presented in the current study, but also only during a limited time period, for therapy (e.g., functional electrical therapy, robotic rehabilitation) or training (e.g., simplifying myoelectric training for a complex prosthesis).

The presented system is an example of the sensor fusion approach to the control of prosthetic devices [34]. In the current system, control was implemented by combining visual information and myoelectric commands. This approach could be extended further. The 3D information about the scene and the target object could be integrated with information about the pose of the prosthesis provided by inertial measurement sensors placed on the device, resulting in an adaptive system that can reconfigure automatically depending on the side and angle of 'attack'. For example, the hand aperture could readjust depending on the side the user is approaching the object or in the case the hand is equipped with wrist rotator and/or flexion/extension, the system can control the hand orientation. The future steps will be system improvement, in terms of more functions and practicality, followed by an evaluation in the specific context and on the actual target population, i.e., amputees or patients, depending on the selected application. The goal of these tests will be to assess the usability, acceptance and finally the efficiency of the proposed interface.

We believe that there are many potential benefits of using the AR interface versus normal visual feedback or tactile stimulation, and investigating this will be the topic of the studies to follow. For example, as a separate study, we plan to perform an in-depth analysis of the performance and possible applications of proposed AR feedback concepts. Specifically, one of the goals would be to compare the regular open-loop (visual inspection) and closed-loop (AR feedback) prosthesis control in more complex real-life scenarios (e.g., cluttered environment with occlusions, adjusting the hand while reaching for the object). The current AR interface could also be easily extended to feedback additional signals. Specifically, the AR channel could be used for the closed-loop control of the grasping force or prosthesis orientation. For example, the grasping force could be visualized as a vertical bar in the peripheral visual field with the height of the bar being proportional to the force amplitude. These developments are also something that we plan to address in future studies, as we believe they are the key for unlocking the full potential of the AR feedback interface.

We are well aware that there are drawbacks that could jeopardize some of the potential applications. For example, when applied in prosthetic or orthotic scenarios, the system could provide simple and effective control of a complex device, but at the expense of adding additional components. In the current version, AR glasses with stereo camera pair have to be worn by the subject. For the user of a prosthesis, this could be an additional nuisance that could further compromise the already sensitive process of device acceptance. However, as always, this ultimately depends on the cost benefit ratio: if the user gets a complex multi-degree of freedom prosthesis that can react to a simple command by automatically reaching and grasping for the desired object, we could expect that he/she would likely be much more eager to wear an additional component. In fact, the control scheme proposed here was filed as a joint patent [59] with our industrial partner (Otto Bock Healthcare GmbH, Vienna, AT), one of the leading manufacturers of prosthetic equipment. Importantly, there are some recent technological developments that could significantly improve the system appearance, thereby overcoming the present drawbacks. Finally, this issue is less likely to be a serious obstacle when considering the potential short term system application (therapy or training). There are studies reporting the application of virtual reality equipment in patients with paralysis [60] as well as in amputees [61]. In conclusion, this study presented a set of novel methods and demonstrated their feasibility using a first developed prototype. The next steps are to further improve the system (ongoing work) and then to benchmark the novel approach against the current state-of-the-art prosthetic systems (semiautomatic versus manual myoelectric control, normal visual and/or tactile feedback versus AR feedback).

\section{Acknowledgments}

We acknowledge financial support by the German Ministry for Education and Research (BMBF) via the Bernstein Focus Neurotechnology (BFNT) Göttingen (grant 1GQ0810) (DF) and the Italian Ministry of Education University and Research, under the FIRB-2010 MY-HAND Project (RBFR10VCLD). 


\section{Appendix. Control flow in detail}

Table A1. Control flow.

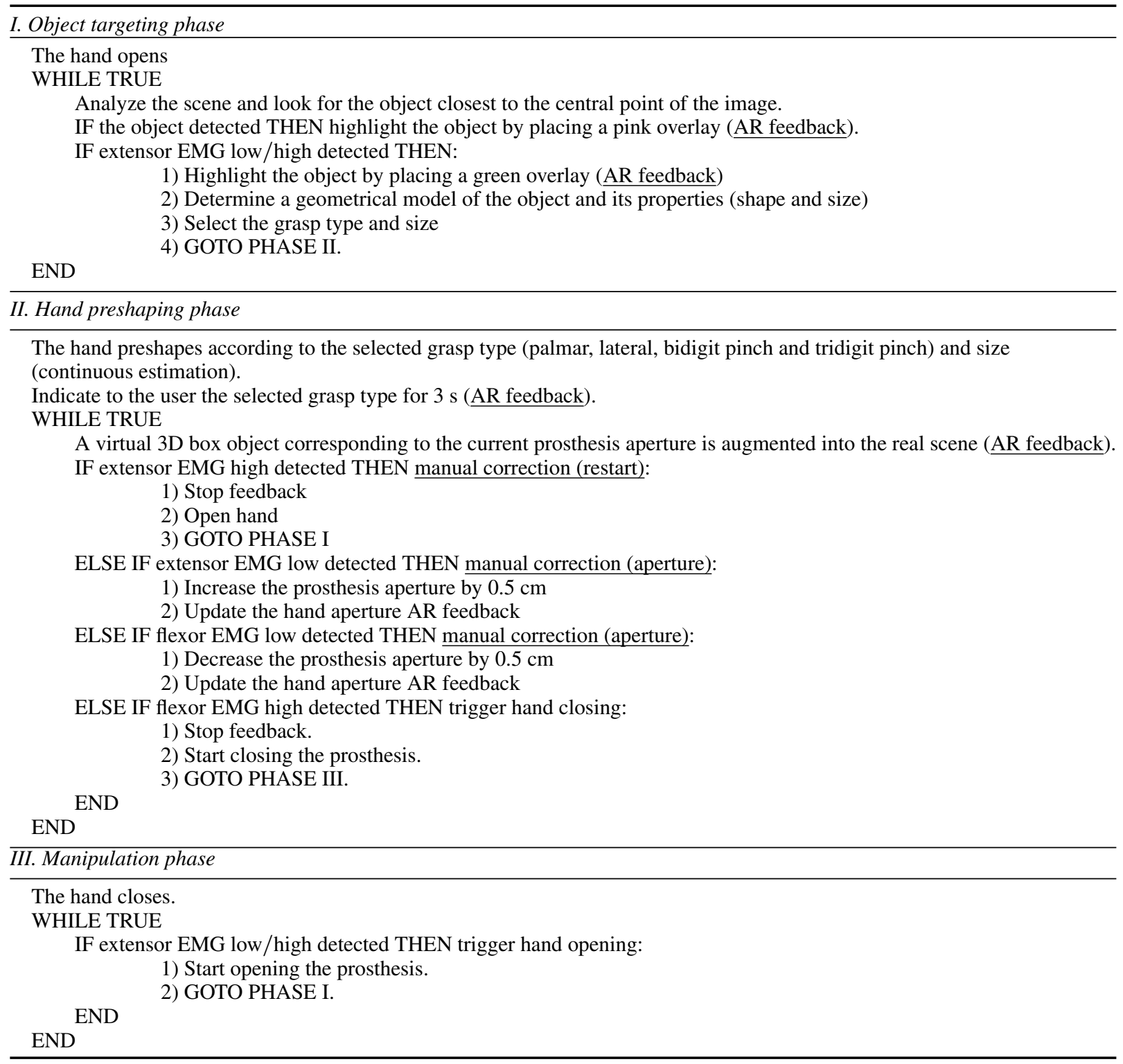

\section{References}

[1] MacKenzie C and Iberall T 2010 The Grasping Hand (Amsterdam: Elsevier)

[2] Bootsma R J and van Wieringen P C W 1992 Spatio-temporal organisation of natural prehension Hum. Mov. Sci. $11205-15$

[3] Connolly J D and Goodale M a 1999 The role of visual feedback of hand position in the control of manual prehension Exp. Brain Res. 125 281-6

[4] Popović M B 2003 Control of neural prostheses for grasping and reaching Med. Eng. Phys. 25 41-50

[5] Balasubramanian S, Klein J and Burdet E 2010 Robot-assisted rehabilitation of hand function Curr. Opin. Neurol. 23 661-70

[6] Cipriani C, Controzzi M and Carrozza M C 2011 The SmartHand transradial prosthesis J. Neuroeng. Rehabil. 829
[7] Heo P, Gu G M, Lee S, Rhee K and Kim J 2012 Current hand exoskeleton technologies for rehabilitation and assistive engineering Int. J. Precis. Eng. Manuf. 13 807-24

[8] Touch Bionics iLimb 2014 www.touchbionics.com/products/ active-prostheses/

[9] Bebionic Hand 2014 http://bebionic.com/the_hand

[10] Parker P, Englehart K and Hudgins B 2006 Myoelectric signal processing for control of powered limb prostheses J. Electromyogr. Kinesiol. 16 541-8

[11] Xiao R and Ding L 2013 Evaluation of EEG features in decoding individual finger movements from one hand Comput. Math. Methods Med. 2013243257

[12] Pistohl T, Schulze-Bonhage A, Aertsen A, Mehring C and Ball T 2012 Decoding natural grasp types from human ECoG NeuroImage 59 248-60

[13] Carrozza M C, Persichetti A, Laschi C, Vecchi F, Lazzarini R, Vacalebri P and Dario P 2007 A wearable biomechatronic 
interface for controlling robots with voluntary foot movements IEEE/ASME Trans. Mechatronics 12 1-11

[14] Johansen D, Popović D B, Struijk L N S A, Sebelius F and Jensen S 2012 A novel hand prosthesis control scheme implementing a tongue control system Int. J. Eng. Manuf. 214

[15] Controzzi M, Cipriani C, Popovic D B and Carrozza M C 2013 Controlling hand-assistive devices: utilizing electrooculography as a substitute for vision IEEE Robot. Autom. Mag. 20 40-52

[16] Asghari Oskoei M and Hu H 2007 Myoelectric control systems-a survey Biomed. Signal Process. Control 2 275-94

[17] Peerdeman B et al 2011 Myoelectric forearm prostheses: state of the art from a user-centered perspective J. Rehab. Res. Develop. 48 719-37

[18] Tenore F V G, Ramos A, Fahmy A, Acharya S, Etienne-Cummings R and Thakor N V 2009 Decoding of individuated finger movements using surface electromyography IEEE Trans. Biomed. Eng. 56 1427-34

[19] Cipriani C, Antfolk C, Controzzi M, Lundborg G, Rosen B, Carrozza M C and Sebelius F 2011 Online myoelectric control of a dexterous hand prosthesis by transradial amputees IEEE Trans. Neural Syst. Rehabil. Eng. $19260-70$

[20] Micera S et al 2011 Decoding of grasping information from neural signals recorded using peripheral intrafascicular interfaces J. Neuroeng. Rehabil. 853

[21] Baker J J, Scheme E, Englehart K, Hutchinson D T and Greger B 2010 Continuous detection and decoding of dexterous finger flexions with implantable myoelectric sensors IEEE Trans. Neural Syst. Rehabil. Eng. 18 424-32

[22] Szeto A Y and Saunders F A 1982 Electrocutaneous stimulation for sensory communication in rehabilitation engineering IEEE Trans. Biomed. Eng. 29 300-8

[23] Kaczmarek K A, Webster J G, Bach-y-Rita P and Tompkins W J 1991 Electrotactile and vibrotactile displays for sensory substitution systems IEEE Trans. Biomed. Eng. 38 1-16

[24] Patterson P E and Katz J A 1992 Design and evaluation of a sensory feedback system that provides grasping pressure in a myoelectric hand J. Rehabil. Res. Dev. 29 1-8

[25] Meek S G, Jacobsen S C and Goulding P P 1989 Extended physiologic taction: design and evaluation of a proportional force feedback system J. Rehabil. Res. Dev. 26 53-62

[26] Scott R N, Brittain R H, Caldwell R R, Cameron A B and Dunfield V A 1980 Sensory-feedback system compatible with myoelectric control Med. Biol. Eng. Comput. 18 65-69

[27] Pylatiuk C, Kargov A and Schulz S 2006 Design and evaluation of a low-cost force feedback system for myoelectric prosthetic hands J. Prosthet. Orthot. 18 57-61

[28] Cipriani C, D’Alonzo M and Carrozza M C 2012 A miniature vibrotactile sensory substitution device for multifingered hand prosthetics IEEE Trans. Biomed. Eng. 59 400-8

[29] D'Alonzo M, Dosen S, Cipriani C and Farina D 2013 HyVE: hybrid vibro-electrotactile stimulation for sensory feedback and substitution in rehabilitation IEEE Trans. Neural Syst. Rehabil. Eng. 22 290-301

[30] Dhillon G S and Horch K W 2005 Direct neural sensory feedback and control of a prosthetic arm IEEE Trans. Neural Syst. Rehabil. Eng. 13 468-72

[31] Karnati N, Kent B A and Engeberg E D 2013 Bioinspired sinusoidal finger joint synergies for a dexterous robotic hand to screw and unscrew objects with different diameters IEEE/ASME Trans. Mechatronics 18 612-23

[32] Malhotra M, Rombokas E, Theodorou E, Todorov E and Matsuoka Y 2012 Reduced dimensionality control for the ACT hand IEEE Int. Conf. on Robotics and Automation pp 5117-22

[33] Matrone G C, Cipriani C, Carrozza M C and Magenes G 2012 Real-time myoelectric control of a multi-fingered hand prosthesis using principal components analysis J. Neuroeng. Rehabil. 940

[34] Dosen S, Muller K-R and Farina D 2012 Myoelectric control of artificial limbs-is there a need to change focus? IEEE Signal Process. Mag. 29 150-2

[35] Dosen S, Cipriani C, Kostić M, Controzzi M, Carrozza M C and Popović D B 2010 Cognitive vision system for control of dexterous prosthetic hands: experimental evaluation J. Neuroeng. Rehabil. 742

[36] Tomovic R and Boni G 1962 An adaptive artificial hand IRE Trans. Autom. Control 7 3-10

[37] Tomovic R, Bekey G and Karplus W 1987 A strategy for grasp synthesis with multifingered robot hands Proc. IEEE Int. Conf. on Robotics and Automation vol 4 pp 83-89

[38] Chappell P H, Nightingale J M, Kyberd P J and Barkhordar M 1987 Control of a single degree of freedom artificial hand J. Biomed. Eng. 9 273-7

[39] Otto Bock Sensor Hand Speed 2014 www.ottobock.de/cps/rde/ xchg/ob_com_en/hs.xsl/3652.html

[40] Engeberg E D and Meek S G 2013 Adaptive sliding mode control for prosthetic hands to simultaneously prevent slip and minimize deformation of grasped objects IEEE/ASME Trans. Mechatronics 18 376-85

[41] Wettels N, Parnandi A R, Loeb G E and Sukhatme G S 2009 Grip control using biomimetic tactile sensing systems IEEE/ASME Trans. Mechatronics 14 718-23

[42] Light C M, Chappell P H, Hudgins B and Engelhart K 2002 Intelligent multifunction myoelectric control of hand prostheses J. Med. Eng. Technol. 26 139-46

[43] Cipriani C, Zaccone F, Micera S and Carrozza M C 2008 On the shared control of an EMG-controlled prosthetic hand: analysis of user-prosthesis interaction IEEE Trans. Robot. 24 170-84

[44] Corbett E A, Körding K P and Perreault E J 2013 Real-time evaluation of a noninvasive neuroprosthetic interface for control of reach IEEE Trans. Neural Syst. Rehabil. Eng. 21 674-83

[45] Kragic D, Miller A T and Allen P K 2001 Real-time tracking meets online grasp planning ICRA'01: Proc. IEEE Int. Conf. on Robotics and Automation vol 3 pp 2460-5 (Cat. No.01CH37164)

[46] Google Glass Project 2014 www.google.com/glass/start/

[47] Geiger A, Roser M and Urtasun R 2010 Computer Vision-ACCV (Lecture Notes in Computer Sciences vol 6492) (Berlin: Springer)

[48] Bouguet J-Y 2013 Camera Calibration Toolbox for Matlab www.vision.caltech.edu/bouguetj/calib_doc/

[49] Fischler M A and Bolles R C 1981 Random sample consensus: a paradigm for model fitting with applications to image analysis and automated cartography Commun. ACM 24 381-95

[50] Cutkosky M R 1989 On grasp choice, grasp models, and the design of hands for manufacturing tasks IEEE Trans. Robot. Autom. 5 269-79

[51] Siebert J P and Cyganek B 2009 An Introduction to 3D Computer Vision Techniques and Algorithms (New York: Wiley) p 504

[52] Hutchinson S, Hager G D and Corke P I 1996 A tutorial on visual servo control IEEE Trans. Robot. Autom. 12 651-70 
[53] Childress D S 1980 Closed-loop control in prosthetic systems: historical perspective Ann. Biomed. Eng. $8293-303$

[54] The da Vinci $®$ Surgical System 2014 www.davincisurgery.com/da-vinci-surgery/ da-vinci-surgical-system/

[55] Prime Sense Sensors 2014 www.primesense.com/

[56] Creative Senz3D 2014 http://de.creative.com/p/web-cameras/ creative-senz $3 \mathrm{~d}$

[57] PCL 2014 http://pointclouds.org/

[58] Ciocarlie M T and Allen P K 2009 Hand posture subspaces for dexterous robotic grasping Int. J. Rob. Res. 28 851-67
[59] Dosen S, Markovic M, Popović D B, Farina D and Graimann B Sensor fusion for control of upper limb protheses: integration of myoelectric control with stereovision, augmented reality and inertial sensing Patent number EP 13171671.42013

[60] Subramanian S, Knaut L A, Beaudoin C, McFadyen B J, Feldman A G and Levin M F 2007 Virtual reality environments for post-stroke arm rehabilitation J. Neuroeng. Rehabil. 420

[61] Murray C D, Pettifer S, Howard T, Patchick E L, Caillette F, Kulkarni J and Bamford C 2007 The treatment of phantom limb pain using immersive virtual reality: three case studies Disabil. Rehabil. 29 1465-9 


\section{APPENDIX 2: SENSOR FUSION AND COMPUTER VISION FOR CONTEXT-AWARE CONTROL OF A MULTI DEGREE-OF- FREEDOM PROSTHESIS}


Sensor Fusion for Closed-loop Control of Upper-limb Prostheses 
Sensor fusion and computer vision for context-aware control of a multi degree-of-freedom prosthesis

This content has been downloaded from IOPscience. Please scroll down to see the full text.

2015 J. Neural Eng. 12066022

(http://iopscience.iop.org/1741-2552/12/6/066022)

View the table of contents for this issue, or go to the journal homepage for more

Download details:

IP Address: 134.76.10.66

This content was downloaded on 11/02/2016 at 21:01

Please note that terms and conditions apply. 


\title{
Sensor fusion and computer vision for context-aware control of a multi degree-of-freedom prosthesis
}

\author{
Marko Markovic ${ }^{1}$, Strahinja Dosen $^{2}$, Dejan Popovic ${ }^{3}$, \\ Bernhard Graimann ${ }^{1}$ and Dario Farina ${ }^{2,4}$ \\ ${ }^{1}$ Department of Translational Research and Knowledge Management, Otto Bock HealthCare GmbH, \\ D-37115 Duderstadt, Germany \\ ${ }^{2}$ Department of NeuroRehabilitation Engineering, Bernstein Focus Neurotechnology Göttingen, Bernstein \\ Center for Computational Neuroscience, University Medical Center Göttingen, Georg-August University, \\ D-37075 Göttingen, Germany \\ ${ }^{3}$ Faculty of Electrical Engineering, University of Belgrade, Bulevar kralja Aleksandra 73, 11020 Belgrade, \\ Serbia
}

E-mail: marko.markovic@ottobock.de, strahinja.dosen@bccn.uni-goettingen.de,dbp@etf.rs, bernhard. graimann@ottobock.de and dario.farina@bccn.uni-goettingen.de

Received 2 March 2015, revised 25 September 2015

Accepted for publication 2 October 2015

Published 4 November 2015

\begin{abstract}
Objective. Myoelectric activity volitionally generated by the user is often used for controlling hand prostheses in order to replicate the synergistic actions of muscles in healthy humans during grasping. Muscle synergies in healthy humans are based on the integration of visual perception, heuristics and proprioception. Here, we demonstrate how sensor fusion that combines artificial vision and proprioceptive information with the high-level processing characteristics of biological systems can be effectively used in transradial prosthesis control. Approach. We developed a novel context- and user-aware prosthesis (CASP) controller integrating computer vision and inertial sensing with myoelectric activity in order to achieve semi-autonomous and reactive control of a prosthetic hand. The presented method semiautomatically provides simultaneous and proportional control of multiple degrees-of-freedom (DOFs), thus decreasing overall physical effort while retaining full user control. The system was compared against the major commercial state-of-the art myoelectric control system in ten able-bodied and one amputee subject. All subjects used transradial prosthesis with an active wrist to grasp objects typically associated with activities of daily living. Main results. The CASP significantly outperformed the myoelectric interface when controlling all of the prosthesis DOF. However, when tested with less complex prosthetic system (smaller number of DOF), the CASP was slower but resulted with reaching motions that contained less compensatory movements. Another important finding is that the CASP system required minimal user adaptation and training. Significance. The CASP constitutes a substantial improvement for the control of multi-DOF prostheses. The application of the CASP will have a significant impact when translated to real-life scenarious, particularly with respect to improving the usability and acceptance of highly complex systems (e.g., full prosthetic arms) by amputees.
\end{abstract}

S Online supplementary data available from stacks.iop.org/JNE/12/066022/mmedia

\footnotetext{
4 Author to whom any correspondence should be addressed.
} 
Keywords: sensor fusion, context awareness, user awareness, reactive control, semi-autonomous, upper limb prosthesis, control of grasping

\section{Introduction}

Human hands are highly dexterous manipulators that integrate a variety of somatosensory and motor systems with the complex musculoskeletal structure in order to generate reaching and grasping movements [1]. During this motor task, the hand is transported to an appropriate location in the vicinity of the object and then oriented and preshaped conveniently to grasp the object by forming an optimal opposition space for a stable grip [1, 2]. Vision provides critical input for the planning and execution of hand transport and prehension, since it allows the nervous system to estimate the extrinsic properties of the target object. The perception of the object's location, size and shape, and orientation with respect to the environment enables the brain to plan the movement by selecting an appropriate reach and grasp strategy. Vision provides feedback during the execution of the movement [35] to allow for corrections, especially in the late phase when the object is approached. This closed-loop operates continuously, providing the flexibility and adaptability that are characteristic of human grasping, where movement planning and execution can be modified at any moment in time to better adapt to the current context. One characteristic example is the change in orientation and preshape during the target approach phase in response to the decision to grasp the object from a different side.

Since we are so heavily dependent on our hands, their loss due to amputation is a traumatic experience with devastating psychophysical effects, dramatically shaping the way affected individuals interact with their environment and others. It has been estimated that in the US alone there are 541000 people living with the loss of an upper limb [6]. The profound negative impact that amputation has on a person's life can (to some extent) be alleviated through the adoption of a hand prosthesis, acting as a partial morphological and functional substitute for the lost limb.

Prosthetic systems have clearly improved over time, with the first devices being simple cosmetic replacements, later developing into passive body-powered mechanical systems before finally being transformed into actuated, battery-powered devices controlled via myoelectric signals [7] approximately 60 years ago. Nowadays, there is a great variety of active systems offering very different functionality [8]: from simple single-degrees-of-freedom (DOF) grippers (e.g., Sensor Hand Speed developed by Otto Bock [9]) over multigrasp systems with an active wrist (e.g., Michelangelo Hand by Otto Bock [10]) to highly dexterous multi-DOF devices closely replicating the structure (number of DOFs) and capabilities (grasping patterns) of the human limbs (e.g., i-Limb developed by Touch Bionics, DARPA's prosthetic arm [11, 12]).

Man-machine interfaces for prosthesis control have not advanced as rapidly as robotic technology [13]. Therefore, most commercially available devices still implement classic sequential and proportional control, which is the first concept proposed for myocontrol [13]. This is partially due to the fact that most transradial prostheses have a small number of DOFs. In these SOA systems the user has to switch between the DOFs, adjusting them one by one, which is a tedious and non-intuitive process [14]. In research, pattern recognition has been extensively tested as a method to improve prosthesis control by allowing the user to select from a predefined set of prosthesis commands through generating an appropriate pattern of muscle activity or other biosignals (e.g., EEG $[15,16]$, voice [17], foot pressure [18]). Despite the promising laboratory results, pattern recognition has so far had only limited translation into clinical applications, mainly due to a lack of robustness. There is only one recently presented, commercially available system advocating this approach (COAPT [19]). Contrary to pattern recognition, in which the user still needs to switch between a limited set of classes, biologically-inspired invasive [20, 21] and non-invasive [2224] methods for direct simultaneous and proportional control of multiple DOFs are being developed with increasing effort.

The current approaches for myocontrol share the same overall structure. As explained above, human grasping proceeds through a sequence of phases, from planning to execution, and involves the integration of sensory information from different sources (e.g., vision and proprioception). In the classic control scheme, the user is responsible for most of the steps, including context assessment, grasp planning, and the generation of control biosignals, while the artificial controller is at the end of the chain, i.e., it acquires the signals and translates them into prosthesis actions. Our research, presented in this paper, advocates an alternative approach that can be classified as symbiotic. Namely, in natural settings, decisions are made on the basis of multisensory information. In the context of movement control the sensors used are vision, muscle spindles, Golgi tendon organs, joint receptors, skin receptors, and the overall neural network at several levels of the central nervous system. This is to say that, apart from capturing myoelectric signals, there are many other sensors that can be used to assist in the control of grasping, as demonstrated in robotics research $[25,26]$. If equipped with such sensors, the prosthesis controller can emulate the highlevel processes traditionally considered the responsibility of the user. By fusing information from different sources, the controller is also able to detect and analyze the current context, plan the grasping action, and simultaneously and proportionally control multiple DOFs available in the prosthesis.

The concept of semi-autonomous control of upper limb prostheses was developed long ago [27-29]. In the prosthesis presented in [28], one of the two available grasp types (pinch or lateral) could be selected automatically depending on the point of first contact with the object as detected by touch sensors. Similarly, in [29] a conceptual solution for triggering the automatic wrist pronation/supination by monitoring the forearm inclination was presented. The idea was to detect the 
intent of the user to rotate the hand by detecting the start of the compensatory movement (e.g., forearm rotation, shoulder abduction/adduction) and then complete the motion automatically, implementing essentially a pronation/supination amplifier [30]. In [31] a full arm prosthesis was envisioned to be operated as a state machine triggering different autonomous functions, such as maintaining constant orientation of the object during transport using inertial sensing (e.g., to prevent spilling of liquid from a glass). Some aspects of the semi-autonomous control concept have also been demonstrated in several subsequent developments [32-35]. In these systems, the user delivered high-level commands (e.g., grasp, squeeze, hold), which the prosthesis would implement automatically based on input provided by the embedded prosthesis sensors (e.g., position, touch). Finally, a simplified form of semi-autonomous control has been translated into a commercially available system with automatic slip prevention [9].

More recently, the control methods were presented based on the fusion of signals from multiple sources. For example, gaze direction was assessed using electrooculography or eye tracking and combined with a brain-computer interface (BCI) or computer vision in order to guide an orthosis [36] or a robotic exoskeleton [37, 38] towards the target. Electrooculography was also employed [39] to communicate the object size to the prosthesis controller as the user scanned the object perimeter using his/her gaze. The controller would then automatically select the grasp type and size suitable for grasping the object. Sensor fusion using electrophysiological signals as well as pressure sensors and inertial units was applied to improve intention detection in the classic patternrecognition- and regression-based manual control systems [40]. One recently presented system [41] combined a BCI with gaze tracking and computer vision in order to trigger and automatically guide the reach-grasp-drop motion of the modular upper limb prosthesis which is mounted next to the user. Models of shared control are being addressed extensively in robotics literature [42] from a somewhat different perspective with the aim to improve the collaboration between humans and external robotics systems, but the concepts are relevant also in the context of robotic rehabilitation.

These developments imply that the semi-autonomous sensor-fusion approach to the control of assistive systems will likely continue to gain in momentum, especially as these systems are becoming increasingly complex (dexterous hands, full-arm prostheses). This is also clearly emphasized in the relevant strategic documents, such as the Multi-Annual Roadmap for Robotics in Europe [43].

In this study, we demonstrate how the sensor-fusion approach can be exploited to improve the control of a multifunction prosthetic system. More specifically, we present a novel controller equipped with artificial vision and proprioception to perceive the state of the user, the prosthesis and the environment. Based on this information, the controller makes autonomous decisions and automatically configures the prosthesis parameters, simultaneously and proportionally adjusting multiple DOFs according to the task demands and reactively to the user's intentions. Therefore, the method presented here exploits a unique and comprehensive combination of sensing units, comprising myoelectric recording, computer vision, inertial measurements and embedded prosthesis sensors (position and force), to develop a controller endowing a multi-DOF prosthesis with the abilities characteristic for advanced robotic systems. The method is based on sensor fusion which allows for the continuous and simultaneous perception of the user (proprioception), the environment (exteroception) and their interaction, leading to online simultaneous and proportional control of multiple DOFs through context-dependent behavior (e.g., reactive response). To the best of our knowledge, this is the first prosthetic system with such a level of autonomy and component integration. Importantly, this approach should not be considered as a substitute for the existing prosthesis control methods. The aim is to integrate the new ideas and solutions with those methods and enhance them with the new functionality. In the current study, we specifically, demonstrated and evaluated how user and context awareness can enrich the classic myocontrol system, leading to autonomous operation using sensor fusion.

\section{Material and methods}

\subsection{Sensor fusion for context-aware semi-autonomous prosthesis control}

The novel concept is depicted in figure 1. The context-aware sensor-fusion controller (CASP) integrates automatic (ACU, figure 1(1)) and myoelectric (MCU, figure 1(2)) control units. The user employs the MCU for manual control (e.g., a classic sequential multi-DOF scheme [44]) and for triggering of the automatic operation. The ACU comprises the artificial exteroception (AEM, figure 1(3)), artificial proprioception (APM, figure 1(4)), and sensor-fusion (SFM, figure 1(5)) modules, providing for the automatic simultaneous and proportional control of multiple DOFs (e.g., a multi-grasp prosthesis with an active wrist).

The AEM (figure 1(3)) uses computer vision to acquire information about the 3D structure of the scene. It analyzes the scene, segments out the object that is closest to the center of the camera's field-of-view, and estimates its shape, size, and orientation. To accommodate arbitrary objects, the AEM does not rely on the predefined database, but analyzes the extracted cloud of points and approximates the target object using common geometrical models.

The APM (figure 1(4)) receives data from the position sensors embedded into the prosthesis providing the size of the hand aperture and wrist rotation relative to the socket. In addition, an inertial unit is used to track the absolute orientation of the prosthesis with respect to an external coordinate system. Therefore, the APM determines the current state of the hand on the basis of commands sent to the prosthesis (hand to socket angles and aperture) as well as volitional user movements (hand to external reference).

The SFM (figure 1(5)) integrates the outputs of the sensing modules (i.e., the hand and object state) and 


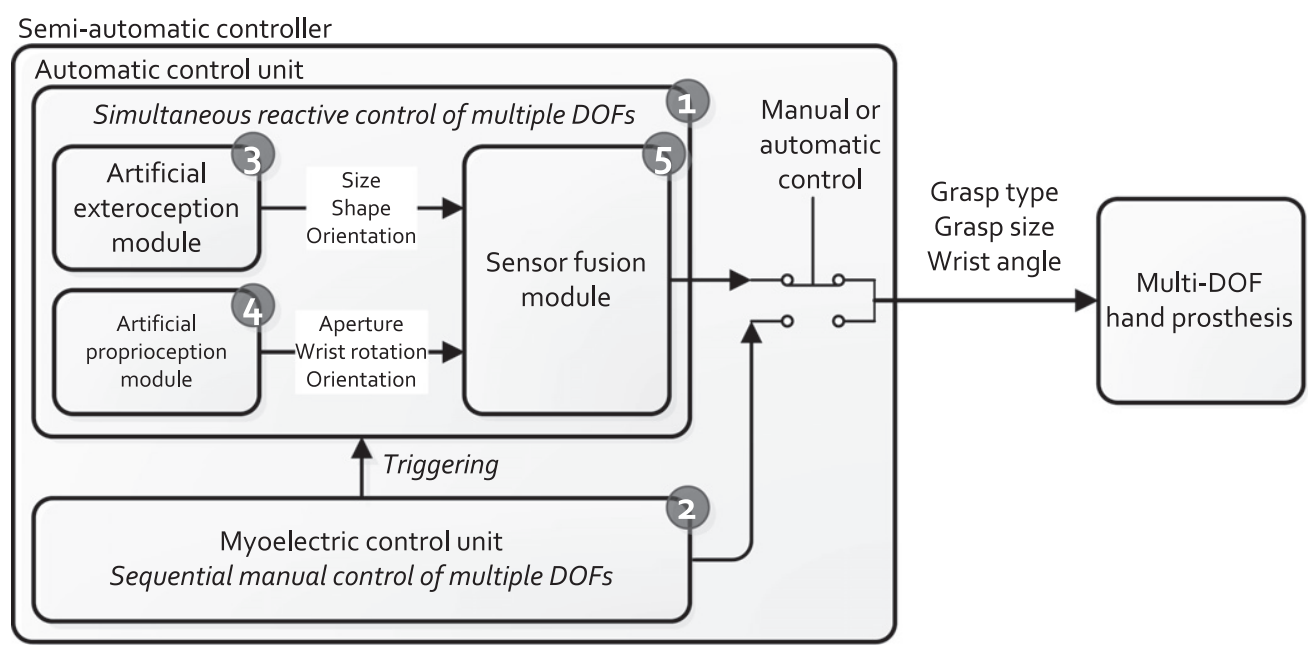

Figure 1. Conceptual scheme of the novel control approach. The semi-automatic controller integrates the myoelectric interface and automatic control unit. The latter provides simultaneous, proportional and reactive control of multiple DOFs (grasp type, size, and wrist rotation) based on the fusion of computer vision (exteroception) and position/orientation measurements (proprioception). The user employs the myoelectric interface for manual control and triggering of the automatic operation.

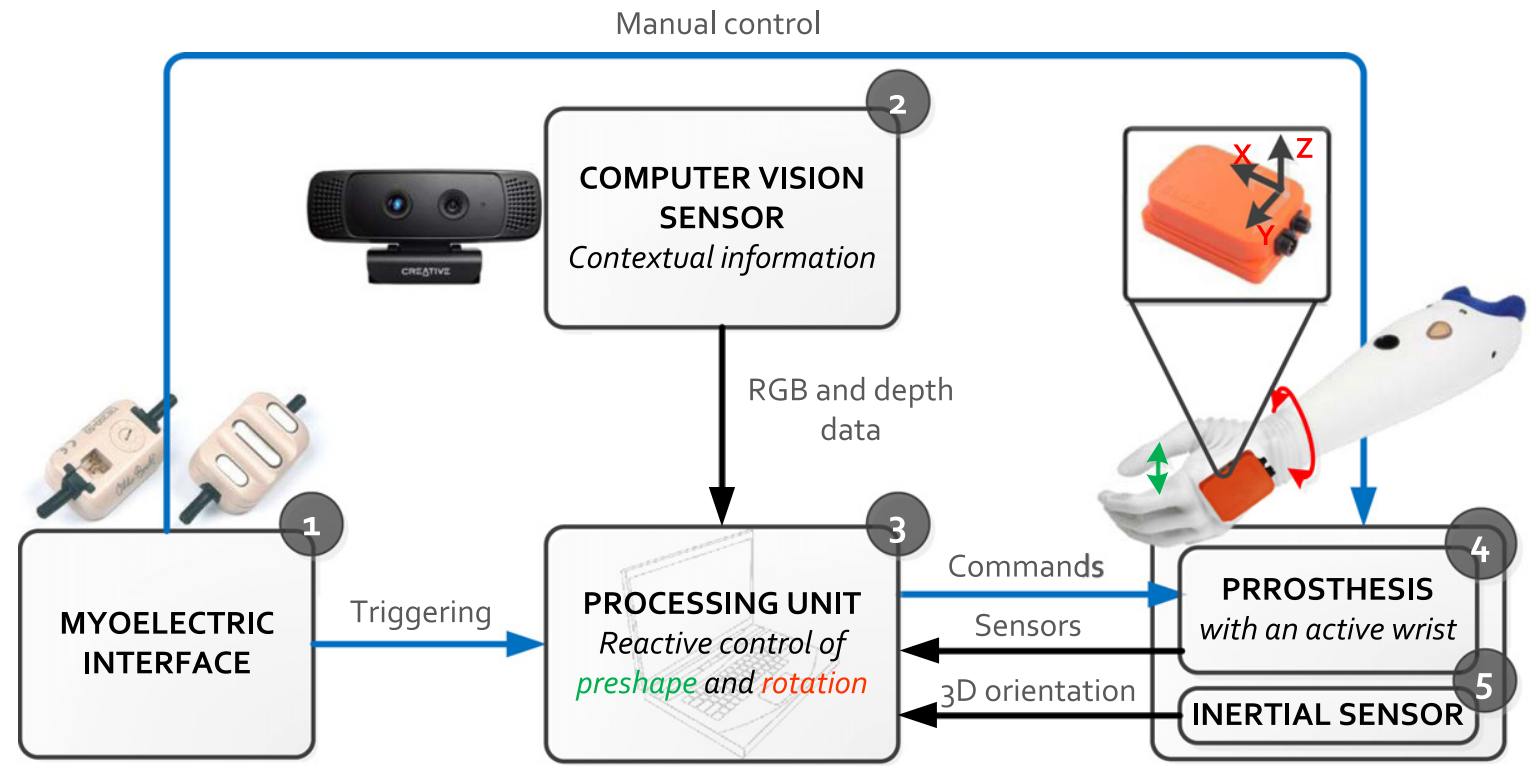

Figure 2. CASP system prototype. When the user generates the trigger command, the processing unit fuses the data acquired from the sensors (i.e., prosthesis aperture, grasp type, orientation, and depth image) in order to perform automatic and real-time updates of the prosthesis parameters. Based on the current state of the prosthesis and the estimated properties of the target object (shape, size, orientation), the prosthesis posture (i.e., grasp type, size, and wrist angle) is configured so that the hand is prepared for grasping the target object. Additionally, the user also has full manual control of the prosthesis through the myoelectric interface, thus being able to correct or fine-tune the autonomous decisions (semi-automatic control).

chooses the grasping strategy. Specifically, the SFM selects the optimal preshape (grasp type and size) and orientation (wrist rotation) for the prosthesis in order to ensure that the prosthesis is suitably configured for grasping the target object.

The semi-automatic controller is continuously active, acquiring and processing the sensor data, and is thus able to reconfigure the prosthesis when necessary (reactive operation). For example, the controller selects a different grasp type and/or readjusts the aperture size if the user decides to change the side from which he/she intends to grasp the object. Importantly, in this control scheme, the system and the user share the responsibility (semi-automatic control). The system automatically controls (e.g., preshapes and rotates) the prosthesis in order to accommodate different objects and situations, as described above, while the user triggers, supervises, corrects and fine-tunes the system decisions through manual control via the MCU. He/she can intervene at any time to manually readjust any of the prosthesis parameters. 


\subsection{System prototype}

The CASP prototype comprises the following components (figure 2): (1) two 13E200 dry EMG electrodes with integrated amplifiers (Otto Bock Healthcare GmbH, Vienna, AT)

(2) Creative Senz3D camera (Creative Technology Ltd, SG) (3) a processing unit (i.e., standard PC with 16GB RAM and four-core i7@2.9 GHz CPU), (4) Michelangelo left-hand prosthesis with a wrist rotator (Otto Bock Healthcare $\mathrm{GmbH}$, Vienna, AT) and (5) an MTx inertial measurement unit (IMU) (Xsens Technologies B.V., Enschede, NL).

The Michelangelo Hand provides simultaneous opening and closing of all fingers with two grasp types (palmar and lateral), as well as and wrist pronation and supination (i.e., three DOFs in total) [10]. The hand was instrumented with three position encoders (thumb, fingers, and wrist) and a single force transducer positioned at the base of the thumb, measuring the hand aperture, grasping force, and hand orientation relative to the socket. The prosthesis was connected to the host PC via a Bluetooth (BT) interface implementing a bidirectional communication protocol running at $100 \mathrm{~Hz}$. This communication channel was used to receive the sensor data from the prosthesis and to send the control commands to the prosthesis.

The IMU was externally attached to the prosthesis (figure 2). The IMU measured the absolute orientation of the prosthetic hand with respect to the laboratory coordinate system, i.e., yaw, roll and pitch angles. The IMU was connected to the battery-powered acquisition and wireless transmission unit (XBus, Xsens Technologies B.V., Enschede, NL) sending data to the host $\mathrm{PC}$ at a sampling rate of $20 \mathrm{~Hz}$.

A Creative Senz3D camera simultaneously acquired color and depth images (RGB-D) [45] and was mounted on the custom-designed support glasses worn by the subject, thus ensuring that the camera was always directed towards the same scene at which the user was currently looking. The acquired image streams were transmitted to the host $\mathrm{PC}$ via a USB port at a $30 \mathrm{~Hz}$ refresh rate and a resolution of $640 \times 480$ pixels for RGB and $320 \times 240$ pixels for depth images, respectively.

The myoelectric interface comprised two active electrodes placed on the forearm over the wrist and hand flexor and extensor muscles. The electrodes with adjustable gain acquired the EMG data at $1 \mathrm{kHz}$ and directly provided the smoothed signals (linear envelopes), as in a commercial system developed by Otto Bock. The linear envelopes were sampled at $100 \mathrm{~Hz}$ and transferred to the host PC via the Bluetooth connection.

The data from the prosthesis, inertial unit, and camera were received by the host $\mathrm{PC}$, where data processing, sensor fusion, and control algorithms were performed. The host PC also provided a user interface for high-level control (e.g., starting and stopping) and system monitoring and setup. The control algorithm was implemented using object-oriented programming in MATLAB 2013a (MathWorks, Natick, US-MA).
The control flow in the prototype was implemented as a finite-state machine in which the state-transitions were triggered manually by the user via the myoelectrical control interface or automatically by external events (i.e., object contact). The operation of the system is depicted in figure 3 . The system is ready and waits for the user input (figure 3(1)). The prosthesis is in the neutral position/preshape, i.e., relative rotation (hand to socket) and absolute rotation (hand to laboratory coordinate system) are close to zero and aperture is $100 \%$. The user directs his head towards the target object resting on the table surface in front of him and indicates his intention to grasp the object by generating a short burst of extensor muscle activity. The system responds by automatically rotating and preshaping the hand conveniently for grasping the object (figure 3(2)). In this particular example, the hand rotated counterclockwise by $90^{\circ}$, preparing to grasp the object from the left side, and simultaneously preshaped into the palmar grasp with an aperture of $30 \%$, thereby adapting to the size of the object. Once the automatic hand preshaping is accomplished, the user assumes full manual control. $\mathrm{He} / \mathrm{she}$ can employ the myoelectric interface to fine-tune and/or correct the automatic decisions or to close the hand and grip the object, via classic sequential and proportional control. Co-contractions are used to switch between the DOFs, while the currently active DOF is operated proportionally, i.e., the velocity of closing/opening, wrist pronation/supination, and grasping force are proportional to the muscle activation level. In addition, the system continues to track the absolute prosthesis orientation in order to be able to assist grasping by reacting to the user's movements. In the current example (figure 3(3)), instead of approaching the object from the side as initially expected, the user decided to grasp the object from the top. Consequently, the user started pronating the prosthesis (compensatory action) and this was detected by the system, which reacted by readjusting the prosthesis, i.e., the hand was automatically rotated so that the palm was in a horizontal (absolute orientation $0^{\circ}$ ); the system thus completed the pronating movement on behalf of the user. Simultaneously, the grasp type was changed to lateral and aperture was set to $20 \%$. Due to this reactive action, there is a theoretical possibility for the user and a prosthesis to enter into a race condition, i.e., the situation in which the CASP and the user would continuously compete to correct each other. This has been prevented through a fundamental design assumption of the CASP system, which is that the user always has priority over automatic control. Therefore, the system stops the automatic adjustment of the prosthesis' posture immediately upon detecting that the user employs the myoelectric interface to manually steer the prosthesis. To grip the object, the user manually closed the prosthesis (figure 3(4)) by activating the flexor muscles (proportional myoelectric control). When the object is contacted, the automatic control is switched off. The operation loop restarts immediately upon the object release. 

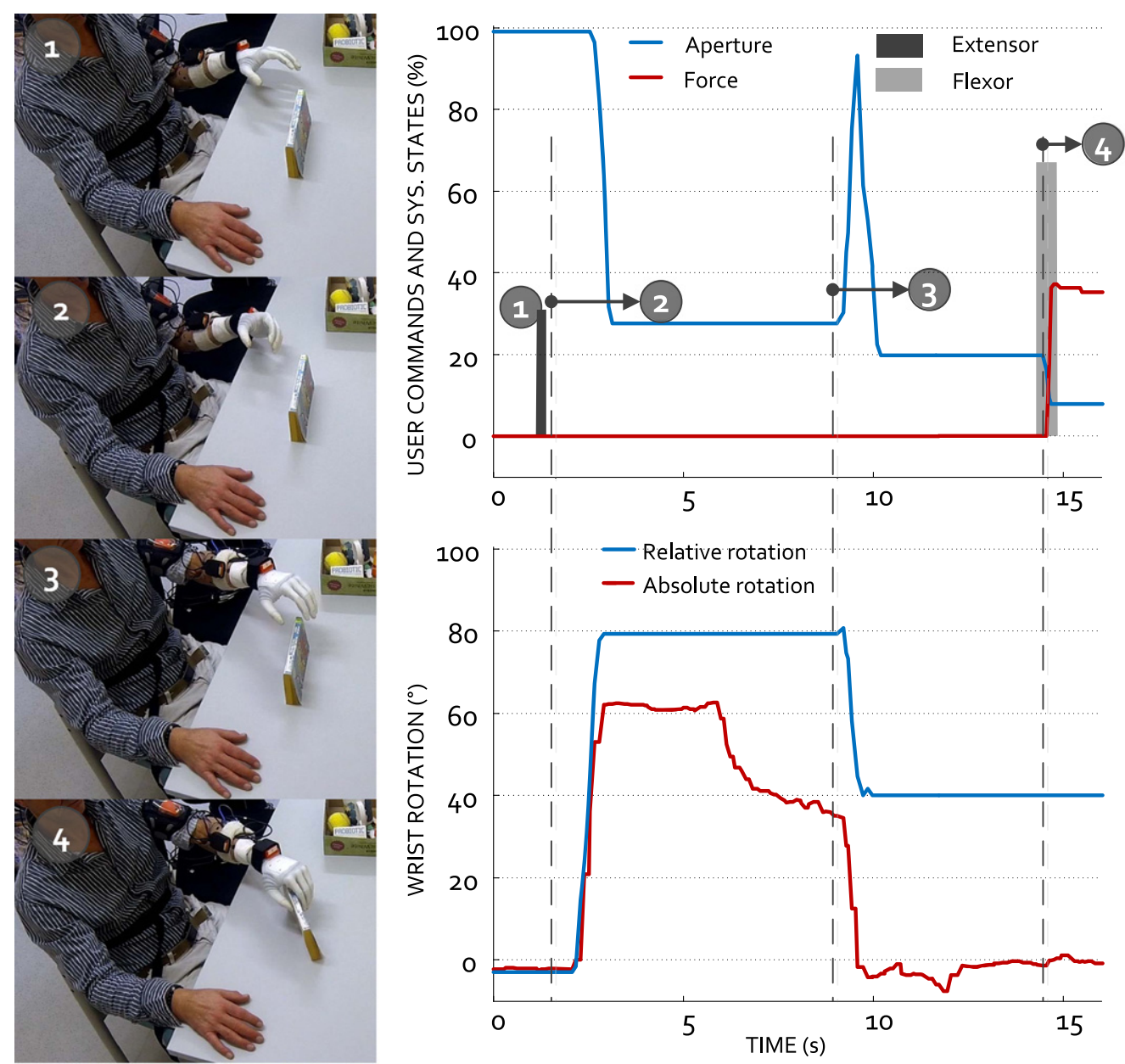

Figure 3. An example of the system workflow. The plot depicts the system states (i.e., absolute/relative rotation, aperture and force of the prosthetic hand) and user commands (i.e., activity of the flexor and extensor muscles). Each number on the snapshot and plot indicates an event triggering a state transition. For the explanation, see the text.

\subsection{Experimental protocol and setup}

The overall goal of the experiment was to test the performance of the proposed CASP system with respect to the SOA manual myoelectric control (MAN).

Subjects. Experimental tests were performed in ten ablebodied subjects $(26 \pm 3 \mathrm{yrs}$, six with prior experience in myoelectric prosthesis control) and one amputee (55 years, 35 years since amputation, active 1-DOF prosthesis user). The local ethics committee approved the study and the subjects signed an informed consent form before starting the experiment.

Procedure. The subjects were seated comfortably in an adjustable chair in front of a table and the system was mounted (figure 4). For able-bodied subjects, the hand was attached to a custom-made ergonomic splint and strapped firmly using Velcro straps to the subjects' left forearm, so that it was positioned directly beneath and perpendicular to the subjects' hand. Due to the space constraints, two EMG electrodes were placed on the contra-lateral arm (figure 4(1)), over the finger and wrist flexor and extensor muscles, which is a common placement for the myocontrol of transradial prostheses. The position was determined by palpating the contracted muscles. For the amputee subject, the prosthesis was mounted using a custom-made socket with integrated electrode placement. Therefore, the myoelectric interface was positioned on the ipsilateral side, as in a real-life application (figure 4(2)). The subjects wore the glasses with the attached depth sensor and the XSens acquisition unit was strapped around the waist and placed on the back.

In addition to the components comprising the CASP system, two extra inertial sensors were placed on the left forearm and upper arm to measure the orientation of the subject's arm. These data were used only for offline analysis to evaluate the employed reach and grasp strategies. In order to simplify the data interpretation the subject's trunk was immobilized using two set of straps to fix the trunk to the chair thus providing a stationary reference for the measurements of arm orientation using inertial sensors. The sensors were positioned so that the local coordinate systems were aligned, with the $X$ - and $Y$-axis pointing in the proximal-distal and median-lateral directions, respectively. The present experimental setup imposes specific constraints (fixed trunk, seating position, prosthetic splint) that likely influence the resulting limb kinematics. Nevertheless, the aim of the present 


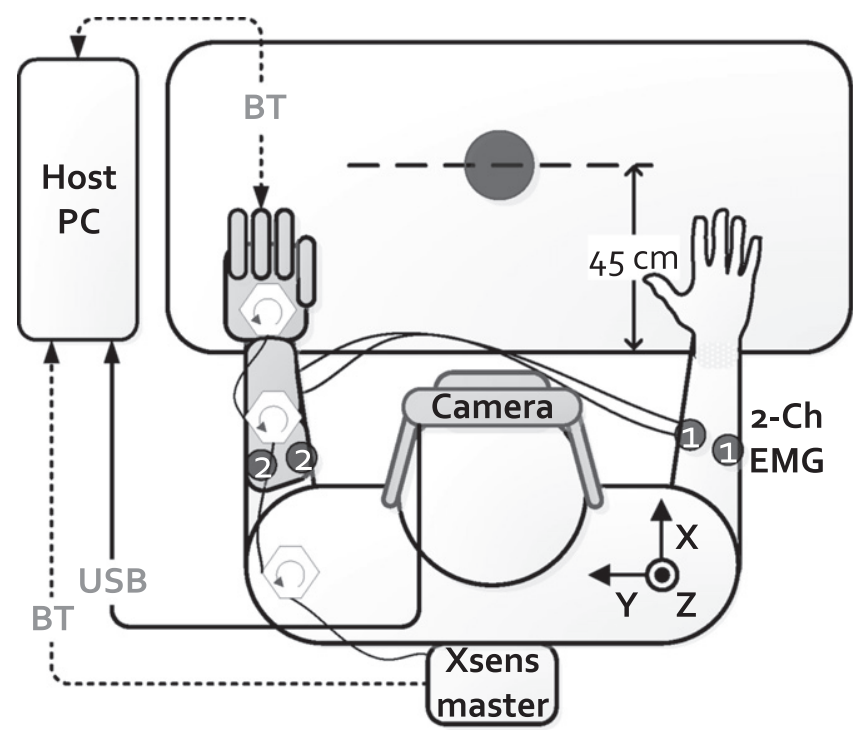

Figure 4. Experimental setup. The user was comfortably seated in front of a table where different objects were presented to him, at the indicated distance. Note that only one inertial sensor, mounted on the prosthetic hand, was used by the sensor-fusion system (other two were used for offline data analysis). Due to the specific prosthesis mounting and space constraints, placement of the EMG electrodes differed for able-bodied (1) and amputee (2) subjects.

study was to capture the kinematic trajectories characteristic for the employed control systems in the given conditions as an additional measure for a more thorough comparison between different solutions.

The outcome of the study is the comparison of the performance between CASP and three, progressively more complex, manual control scenarios MAN $n(n=1,2$, and 3 denotes the number of manually controllable DOFs):

(1) MAN1: proportional control of the prosthesis velocity of closing and opening and grasping force, hand in palmar grasp, wrist orientation fixed in the neutral position.

(2) MAN2: as in MAN1 plus the subjects selected between the palmar or lateral grasps using co-contractions.

(3) MAN3: as in MAN2 plus the subjects proportionally controlled the velocity of wrist rotation (pronation and supination), co-contractions were used to switch between the DOFs in the following order: palmar grasp, lateral grasp, and wrist rotation.

(4) CASP: prosthesis operated as described in section 2.2.

The test comprised training and evaluation session conducted in two consecutive days. In both sessions, the subjects followed the same protocol, i.e., they grasped, lifted, transported and released a set of common daily objects (see table 1) using the four control conditions. The initial (resting) position for the hand and the initial position for the target object were marked on the table surface. A box container to which the objects had to be transported and released after being grasped was also placed on the table, as depicted in figure 4 . The four conditions were performed in a random order and each comprised 17 grasping trials.
The myocontrol parameters were adjusted at the beginning of the sessions. The maximum command to the prosthesis was generated with the muscle activity at approximately $70 \%$ of the maximum voluntary contraction. The attached inertial sensors were calibrated while the subject was holding his arm fully extended, in front of the body and parallel to the table surface.

The aim of the training session was to familiarize the subject with the setup, protocol and tasks to be performed during the experiment. The subjects were explained how to operate the prosthesis using the commercial SOA myocontrol interface and then practiced switching between the active DOFs by generating co-contractions as well as the proportional control of the selected DOF by modulating the level of muscle activity. The subjects were also trained in using CASP system as a whole, including triggering, reactive operation, and the use of manual control to fine-tune/override the system decisions. For each control scenario, the subjects had a short introduction 5-10 min, after which they continued the training by performing the grasping trials.

In each grasping trial, the subjects were presented with a single object and instructed to adjust the prosthesis so that the hand was configured appropriately for grasping the object, as specified in table 1 . There was no time limit for performing the trial. The subjects were instructed to perform the task correctly and as fast as possible. In MAN conditions, this was accomplished by manually operating the available DOFs. In MAN1 and 2, the wrist was inactive and the subjects had to use the proximal arm joints to orient the hand properly for grasping. The grasp type in table 1 was ignored in MAN1 (i.e., all objects grasped using palmar grasp). With CASP, the subjects triggered the system, assessed the outcome of the automatic decisions and corrected the system using manual control when necessary (wrong decision). After the hand was preshaped, the subjects used manual control to close it, lift, transport the object, release it into the container and finally return the prosthesis back to the initial position. The experimenter observed the task execution and the trial was repeated if the gross error has been made and hand has not assumed the correct posture (i.e., the subject or the system employed wrong grasp type or orientation). Concerning the orientation, the absolute precision was not of interest, similar to the real life application where there is a reasonable margin of error under which the task can be successfully accomplished. For example, the user can fine-tune the alignment between the hand and the object using other degrees of freedom (e.g., shoulder joint). Therefore, we deemed that asking the user to control precisely the orientation would be highly artificial (and likely worse in the manual control scenario, where it is done using a naked eye). Instead, the subjects were instructed in general terms to adjust the orientation to complement that of the object, and only if it was completely wrong (e.g., hand vertical and the object horizontal), the experimenter decided to repeat the task. Importantly, the prosthesis posture (grasp type, orientation) was not altered in-between the trials, i.e., the initial posture in the current trial was the posture used to grasp the object in the previous trial. This has been done in order to simulate the real-life usage of the prosthesis, as when 
Table 1. Overview of the test trials, objects used and grasping instructions given.

\begin{tabular}{lllll}
\hline Trial tag & Object description and dimension $(\mathrm{cm})$ & $\begin{array}{l}\text { Object placement on the table } \\
\text { surface }\end{array}$ & Grasp type & $\begin{array}{l}\text { Hand orientation in respect to table } \\
\text { surface }\end{array}$ \\
\hline P1_0_P & Tennis ball $(6.5)$ & Horizontal & Palmar & Parallel \\
P2_90_P & Mug $(9 \times 8)$ & Vertical & Palmar & $90^{\circ}$ supination \\
P3_0_P & Juice-bottle $(169 \times 7.5)$ & Horizontal & Palmar & $\begin{array}{l}\text { Parallel } \\
\text { P3_45_P }\end{array}-$ \\
P3_90_P & - & Leaning $45^{\circ}$ & Palmar & $45^{\circ}$ supination \\
P4_0_P & Tea-box $(139 \times 7.59 \times 6.5)$ & Vertical & Palmar & $90^{\circ}$ supination \\
P4_45_P & - & Horizontal & Palmar & Parallel \\
P4_90_P & - & Leaning $45^{\circ}$ & Palmar & $45^{\circ}$ supination \\
P5_90_P & Espresso cup $(59 \times 5)$ & Vertical & Palmar & $90^{\circ}$ supination \\
L1_0_L & Thin pen $(149 \times 1.5)$ & Vertical & Palmar & $90^{\circ}$ supination \\
L1_45_L & - & Vertical & Lateral & Parallel \\
L2_45_L & Fork $(189 \times 1)$ & Leaning $45^{\circ}$ & Lateral & $45^{\circ}$ pronation \\
L3_0_L & Thick pen $(129 \times 2.5)$ & Leaning $45^{\circ}$ & Lateral & $45^{\circ}$ pronation \\
B1_0_L & Medicine-box $(9 \times 7 \times 1.5)$ & Vertical & Lateral & Parallel \\
B1_90_P & - & Vertical & Lateral & Parallel \\
B2_0_L & Book $(15 \times 9 \times 2)$ & Vertical & Palmar & $90^{\circ}$ supination \\
B2_90_P & - & Vertical & Lateral & Parallel \\
\hline
\end{tabular}

the user grasps objects in succession readjusting the hand from the configuration attained in the last grasp to the one convenient for the next target object. Therefore, even though the task was divided in trials for convenience (e.g., data logging), it could be regarded as an uninterrupted sequence of actions in which the task of the user was to pick up and transport a set of objects. The only exception was if the trial had to be repeated, in which case the posture was reset to the one the hand assumed at the beginning of the trial.

\subsection{Data analysis}

The primary outcome measure was the time to grasp (TTG) an object using a specific control scheme, assessing the efficacy in operating the prosthesis employing that particular control. The TTG was also used to compare the performance between the training and evaluation sessions for the same control scheme to assess if there was an improvement due to the training. The TTG was measured from the start of the trial until the hand contacted the object (force $>$ threshold).

Secondary outcome measures were the shoulder joint angles computed from the inertial data, recorded during the evaluation session only, assessing the arm configuration just before the hand grasped the object $(0.5 \mathrm{~s}$ before contact). They were calculated as the Euler angles of the upper arm with respect to the immobilized trunk.

To assess the system responsiveness, the reaction time (RT) of the CASP to user trigger and reactive response was monitored during the experiment. The former was measured as the time from the detection of the user's trigger until the commands have been sent to the hand and it therefore includes sensor-fusion processing. The RT for the reactive responses comprises the time between the detection of the movement (compensatory rotation) and command transmission. It is important to note that this time is actually already integrated in the TTG performance metric. The trial failure rate was also determined. This index indicated the number of repeated trials, when either the user (MAN1-3) or the system (CASP) made a gross error in adjusting the prosthesis' posture. The gross error referred to an incorrect grasp type or completely wrong orientation (as explained above).

Friedman test was used to assess the statistically significant difference within the group of conditions in the evaluation session, since the data did not pass the test of normality (Liliefor test). For the pairwise comparison, Tukey's honestly significant difference criterion was applied. Finally, to compare the same condition between the training and testing, a Wilcoxon signed-rank test was employed. A $p$ value of 0.05 was selected as the threshold for the statistical significance.

\section{Results}

In total, 1496 trials $(11$ subjects $\times 2$ days $\times 4$ series $\times 17$ trials) were performed. They were allocated evenly between the four control conditions. The results are reported as mean \pm standard deviation and are presented separately for able-bodied and the amputee subject.

Figure 5 shows the average TTG for each of the four test conditions during training and evaluation. During the evaluation session, the TTG in the manual control scenarios (MAN1-3) increased consistently with the number of controllable DOFs, i.e., it was $3.7 \pm 1 \mathrm{~s}$ for MAN1, $4.3 \pm 1.7 \mathrm{~s}$ for MAN2, and then it increased substantially to $11.2 \pm 4.1 \mathrm{~s}$ in MAN3. The differences were statistically significant between all MAN conditions. The TTG with CASP was $5.9 \pm 1.9 \mathrm{~s}$, which was slower than in MAN1 and MAN2 but substantially faster than in MAN3. Using CASP, the subjects could grasp an object in approximately twice less time compared to MAN3. There was virtually no improvement between the training and evaluation sessions with CASP, 


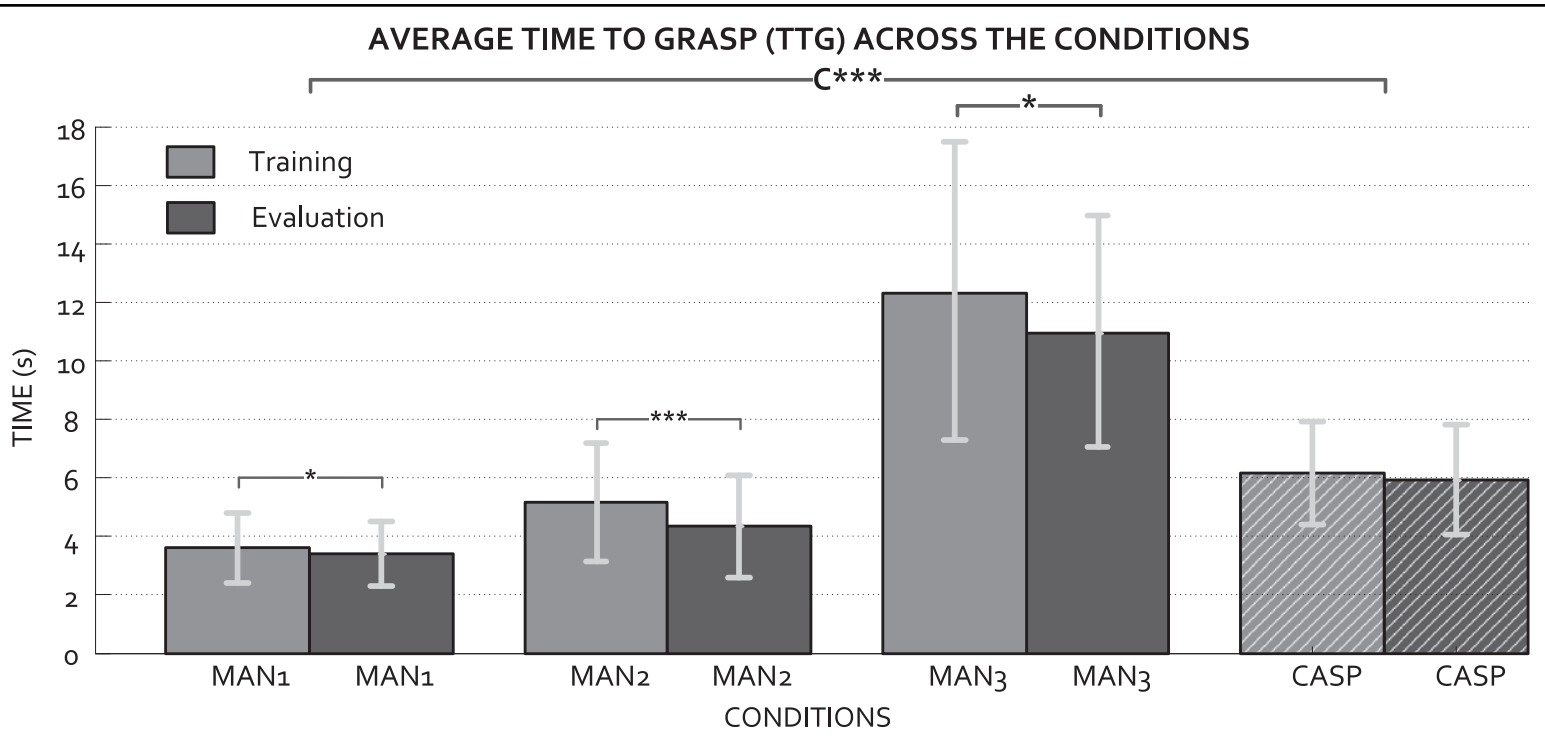

Figure 5. Summary results (mean \pm standard deviation) for average time to grasp (TTG) an object. The statistically significant differences are denoted by a star $\left({ }^{*}, p<0.05 ;{ }^{* *}, p<0.01 ;{ }^{* * *}, p<0.001\right)$ while the symbol ' $\mathrm{C}$ ' indicates that the difference exists across all conditions that were performed within the same session. Notations: MAN $n$-manual control of $n$ DOFs; CASP — context-aware sensor-fusion prosthesis (3 DOFs).

contrary to MAN conditions, which all improved with training.

The RT of the CASP system for user trigger and reactive response were less than 0.75 and $0.1 \mathrm{~s}$, respectively. The cumulative trial failure rate, overall all subjects and trials, was consistently small across all control scenarios: four in MAN2, five in MAN3, and seven in CASP condition, which accounts for less than $1 \%$ of the total number of performed trials.

Similar trends were also observed in the amputee subject where the average TTG was $2.5 \pm 1 \mathrm{~s}, \quad 4.7 \pm 1.8 \mathrm{~s}$, $10 \pm 2.2 \mathrm{~s}$, and $5.5 \pm 1.8 \mathrm{~s}$ for MAN1-3 and CASP conditions respectively. The subject successfully learned how to use the CASP system and, although he was an experienced user of a classic myoelectric prosthesis, the results were similar to the ones obtained for the able-bodied subjects. The CASP system was approximately twice as fast as the manual control for the same number of DOFs (MAN3). The subject reported that the CASP was easy to use and that he liked the approach, especially the fact that the system reactively readjusted the wrist-hand configuration, which added to the overall easiness of the grasp execution.

The summary results for the recorded shoulder-joint angles are given in figure 6(A). The conditions in which there was no wrist rotation control (i.e., MAN1, MAN2) differed significantly in shoulder abduction and external rotation compared to the conditions with manual (MAN3) or automatic (CASP) rotation control. Between MAN1 and 2, the angles were similar, which also held for MAN3 versus CASP.

Figures 6(B) and (C) depicts graphically the arm configurations when the cup positioned horizontally (figure 6(B)) and vertically (figure $6(\mathrm{C})$ ) was grasped using MAN1 (figures 6(B), (C-1)) and CASP (figures 6(B), (C-2)). In MAN1, the user had to perform extensive compensatory movements consisting of either shoulder abduction and external rotation (figure 6(B)) or adduction and internal rotation (figure $6(\mathrm{C})$ ) in order to orient the hand appropriately for grasping the object. The shoulder joint movements were employed in order to compensate for the lack of pronation/ supination at the wrist, and the exact strategy was dependent on object orientation (horizontal versus vertical). On the other side, there were no such over-extensive movements when using CASP and the shoulder angles remained virtually unaffected by the object orientations, since the automatic control adjusted the hand orientation accordingly, using the active wrist joint.

\section{Discussion}

In this study, we have presented a novel concept for the semiautonomous, simultaneous and proportional control of a multi-DOF prosthesis, which is based on fusion of information from a variety of sensing technologies, including computer vision, inertial sensing, position and force sensors, embedded into the prosthesis and myoelectric signal acquisition. These inputs equip the artificial controller with the artificial vision and proprioception for context estimation and autonomous operation, and implement the integration of the biological and artificial control. By exploiting the sensor fusion, the artificial controller is able to emulate the high-level functions that are traditionally regarded as the responsibility of the biological control, such as, grasp planning and execution, and online reactivity to dynamically changing user intentions. At the same time, the status of the user is not compromised, since he/she still has a supreme control over the system.

The system presented here should be considered as an early stage prototype (lab-based device) which is used to demonstrate and evaluate the novel concepts for prosthesis control on able-bodied and amputee subjects. This is an 
AVERAGE SHOULDER ANGLES ACROSS THE CONDITIONS

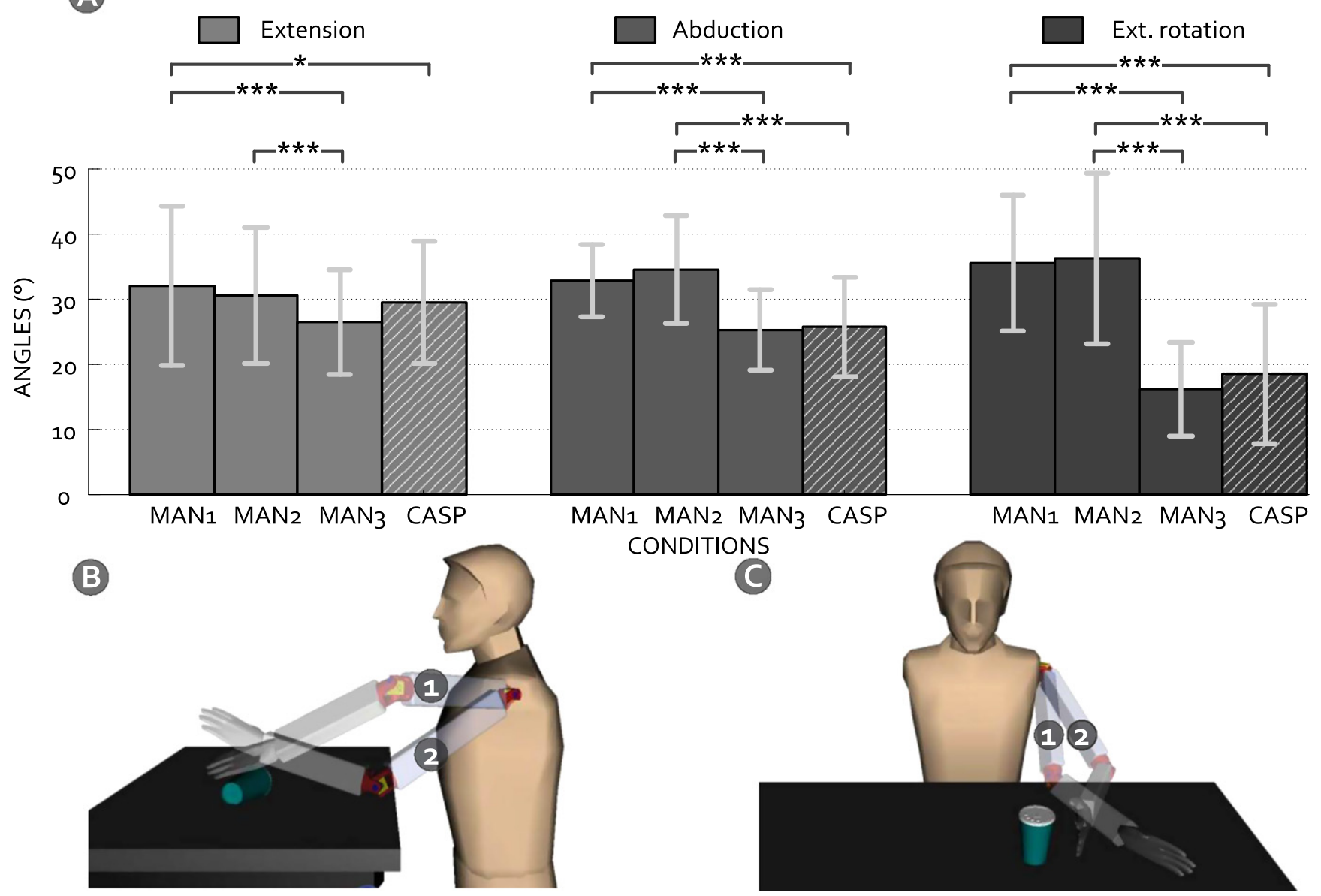

Figure 6. Summary results (mean \pm standard deviation) for average shoulder joint angles (A) across different conditions and 3D model showing the arm positions recorded shortly before the object was grasped (B), and (C). Object placed horizontally (B) and vertically (C) was grasped using MAN1 ((B1) and (C1)) and CASP ((B2) and (C2)) control schemes. Note that the subject employed compensatory maneuvers at the shoulder joint when there was no active wrist rotation ((B) and (C), MAN1), and these movements depended on the orientation of the given object.

important step since the semi-autonomous sensor-fusion approach to prosthesis control opens up a number of possibilities for enhancing the device with automatic functions characteristic for context-aware robotic systems [43]. However, determining which of these functions is feasible and most useful still needs testing. This assessment should identify those functions that deserve to be translated into the future practical version of the system. Even though the current state-of-the-art technologies (e.g., the sensor size [45] and computational requirements) prevent its full integration, the practical relevance of the proposed approach is indicated by the industrial interest in this technology (joint patent application with Otto Bock Healthcare GmbH, Vienna, AT) [46]. Moreover, the wearable technology trends set by the IT industry leaders, i.e., ergonomic glasses integrating inertial units, depth sensors, and augmented reality, are becoming available on the market from a variety of vendors (e.g., Microsoft HoloLens [47], Meta glasses [48]).

The prototype described in the present study is based on our previous work [49-51]. Its performance in correctly estimating the prosthesis aperture and orientation is in the range of $0.75 \pm 1.1 \mathrm{~cm}$ and $9 \pm 5^{\circ}$, respectively, as reported in $[49,52]$. Importantly, it represents a substantial step ahead with respect to the earlier developments, demonstrating for the first time several important concepts realized thanks to the fusion of information from multiple sensors. First, the system integrates the control of an active wrist by combining computer vision (environment analysis) and the measurement of the prosthesis orientation. Second, in addition to assessing the environment, the artificial controller monitors the behavior of the user (his/her movements) via inertial sensing. By integrating this information and the user's voluntary commands, the controller is capable of generating context-dependent decisions, reacting to the dynamically changing state of both the user and the environment. Importantly, the sensor-fusion in the present prototype lays down the basis for a powerful approach, allowing the prosthesis to incorporate many additional intelligent behaviors, which will be tested in the future (see section 4.4). In addition to demonstrating the approach, the present study compares for the first time the performance of this semi-autonomous sensor-fusion prototype to that of the classic manual control (commercial benchmark). The tests 
were conducted for several configurations of the manual interface, determining the pros and cons of the semi-automatic control through a range of functionally relevant assessments.

The fusion of information from multiple sources has been used for decades for grasp planning and execution in industrial and humanoid robotics [53, 54]. However, the control of prostheses is a unique context, since the artificial controller has only a partial control of the system. For example, in the case of a hand prosthesis, the user transports the hand and orients it in space through elbow and shoulder actions, whereas the prosthesis controller operates the wrist and hand closing/opening. This specific scenario on one side relaxes some performance requirements, i.e., the user can compensate for the mistakes of the autonomous controller. On the other hand, it also imposes some specific constraints with respect to typical robotics applications. For example, to properly implement the autonomous actions, the prosthetic controller needs to consider both the prosthesis and the user.

\subsection{Comparison with a commercial myoelectric control}

Single DOF control in MAN1 (hand open/close) was a simple scenario, in which the subjects only had to reach for the object and close the hand, resulting in the smallest TTG overall. However, the subjects had to employ compensatory strategies characterized with the excessive shoulder movements in order to accommodate for the lack of active DOFs (figure 6). In MAN2, the subjects could additionally select between the two grasp types and the control scheme has increased in complexity, as the subjects had to perform the co-contractions to switch between the grasps. This had a statistically significant impact on the overall performance, which slightly deteriorated. However, the addition of a novel grasp-function did not change the reaching strategy, and the same compensatory movements were still present, i.e., the shoulder angles employed in MAN1 and MAN2 were not statistically different. Only in MAN3, in which the wrist was also actively controllable, the overall shoulder angles displayed a significant reduction, especially in abduction and external rotation. This was also obtained during the CASP control condition, meaning that the reaching movements employed in MAN3 and CASP were kinematically similar, i.e., there was no statistical difference between the respective angles. However, although the increased flexibility eliminated the compensatory movements, it also substantially increased the complexity of control since the subjects had to perform tiresome co-contractions several times in a single grasping trial. Consequently, the TTG increased dramatically with respect to MAN1 and 2.

The TTG with the CASP was higher when compared to the MAN1 and 2, but also substantially lower when compared to the MAN3. Therefore, the CASP system is somewhat slower than MAN1 and 2 but results in grasping strategies and arm configurations that are similar to those employed in MAN3, with the benefit that it is substantially faster than the latter. Therefore, when using the full potential of the prosthetic system, the CASP outperforms the myoelectric control significantly. Furthermore, the CASP system was easy to adopt and use, which is demonstrated by the fact that there was no significant improvement in performance between the two experimental sessions (training versus evaluation), which is contrary to MAN conditions.

\subsection{Integration with other methods}

As stated before, the CASP was not designed to compete with the myoelectric interfaces but rather to encapsulate those methods within the semi-autonomous framework and thus enhance their functionality and performance. The present study, for example, demonstrated how adding the CASP on the top of the classic control scheme (commercial benchmark) influenced time efficiency as well as the grasp kinematics. The currently implemented two-channel proportional myocontrol is just one of the many system components that will be improved in the next developments. Future studies will investigate how beneficial would be to integrate the CASP system with advanced myoelectric control schemes, such as methods for simultaneous and proportional myocontrol of multiple DOFs [22, 55]. The concept of the experiment would be similar as in the present study, i.e., the subject performance with an advanced myoelectric interface would be compared to the performance when using the same myoelectric interface integrated within the CASP framework. However, it could be also relevant to compare the CASP against manual control even when the myoelectric interfaces are not the same. In this case, the CASP could allow similar performance as the sophisticated manual control, such as, the multichannel simultaneous and proportional multiple DOF control, but by using a substantially simpler human-machine interfacing, e.g., a two-channel EMG interface triggering automatic simultaneous and proportional control of multiple DOFs. However, these assumptions still need to be confirmed through dedicated experiments.

Similar approach could be also applied in the patients that underwent targeted muscle reinnervation [21] and therefore need to control a full prosthetic arm. Semi-autonomous control could be used to assist direct control through independent signal sources available in these patients. For example, CASP controller could operate the shoulder and elbow joints transporting the hand near the target object, which is a challenging task to perform manually, and then the user could take over and perform the grasp by operating the hand and wrist using direct approach.

Importantly, there are many other possibilities for integration of various manual control methods with the CASP approach, and the aim is to investigate this in the future studies. In addition to controlling a multi-DOoF prosthesis, the presented approach can be extended to other similar applications, e.g., improving the control of an upper-limb rehabilitation robot or functional electrical stimulation. Importantly, the semi-autonomous sensor-fusion control in both prosthetics and rehabilitation robotics can benefit substantially from a large body of work regarding the grasp planning and execution already available in the robotics literature (e.g., visual servoing [53, 54, 56]). However, these 
methods need to be integrated into the context of shared control with its specific constraints and challenges, as explained in section 4 .

\subsection{Sensor fusion and reactive control}

Control based on sensor fusion is a central feature of the proposed system. By integrating the artificial proprioception and vision estimating the orientation of the prosthesis and the object, respectively, we could implement the control of wrist rotation (grasp planning) and an automatic response (artificial reflex) triggered by the change in the approach to the object during hand transport (automatic prosthesis readjustment). The main principle when implementing the autonomously controlled readjustment was that it was synchronized with the users' intentions. In the present prototype, this action was integrated as a reflex, i.e., an automatic response triggered by a discrete sensor input. More specifically, the system monitored the hand orientation (pro/supination axis) and when it detected that the user started rotating, crossing a predefined angular threshold, the system would complete the rotation on behalf of the user as well as readjust the preshape parameters. The threshold was set in the pilot tests to guarantee robust triggering. The system therefore 'amplified' an ongoing movement of the subject, performing the action only when the certainty about the user intention was high. Hence, the reactive behavior was regarded as intuitive for the subjects (verbal report), the subjects easily learned the function, and it did not lead to overshooting of the target or race conditions in any of the trials.

These can be considered as basic actions demonstrating the potential of the sensor fusion for prosthesis control, which can be used to implement many other functional high-level behaviors. For example, by adding inertial sensors to the able hand, in the form of a miniature bracelet or clip attached to clothing, the CASP could adjust the posture of the prosthesis automatically when it detects that the able hand approaches the prosthesis in order to hand over an object. This activity belongs to the class of symmetric bimanual tasks, during which one hand essentially mirrors the orientation of the other. To achieve this, the CASP would read the momentary orientation of the able hand and adjust the prosthesis accordingly. Importantly, this configuration (inertial units placed bilaterally) would open up possibilities for other, more sophisticated scenarios, including automatic intention detection from hand/prosthesis motion as well as automatic support to the other types of bimanual activities. This functionality would be of interest for unilateral amputees, who often use the prosthesis to support the dexterous activities performed by the contralateral (unaffected) side [57].

We hypothesize that the CASP could yield two additional important advantages. First, the automatic functions (e.g., grasp planning) could potentially decrease the cognitive effort of the user. The sensor fusion could allow the user to focus on the task rather than on how to steer the prosthesis. In the current implementation, for example, the user needed to issue only a single, simple command (extensor contraction) and the automatic control would adjust all the prosthesis DOFs on his/her behalf. In MAN3, on the contrary the muscles had to be activated several times, including multiple co-contractions, to achieve the same task. Second, the autonomous operation could facilitate the integration of the prosthesis into the body scheme, since the sensor fusion could improve the coordination between the prosthesis and rest of the body. For example, with automatic control, the user could issue the trigger command and immediately start transporting the hand towards the target. The controller would automatically adjust prosthesis' DOFs during reaching, which resembles a simultaneous evolution of reaching and grasping characteristic for able-bodied subjects [1]. In addition, the artificial reflexes such as reactive readjustment (present study) or future work in bimanual tasks represent the prosthesis actions that are triggered by and synchronized to the volitional movements of the user. However, the decrease in the cognitive effort and the improvement in coordination with CASP are currently only the hypotheses that need to be evaluated objectively via a set of psychometrically validated tools such as NASA Task Load Index [58, 59].

\subsection{Study limitations and future developments}

The CASP approach can be developed in several directions. First, other sensors can be used to monitor the state of the prosthesis and the user and estimate the features of the environment (e.g., laser scanners, ultrasound rangers, time-offlight cameras, RFID tags etc) These sensing elements can be considered and evaluated as the potential information sources for the sensor fusion. For example, RFID tags, or a similar technology such as Bluetooth equipped miniature chips [60], could be used to tag objects in a known environment (e.g., at home). The prosthesis equipped with an integrated RFID reader [61] could detect the tag when approaching the object, read the programmed grip pattern, and preshape automatically [62]. As demonstrated in [63], the tags can be placed also on the user to trigger prosthesis functions each time the user approaches the tag. This approach fits well with the concept proposed in the present study, where additional sensors are placed on the user (e.g., miniature inertial sensing bracelet) to monitor his/her behavior for context-dependent control. Moreover, as shown in [64], RFID tags could be used not only to identify the object and associated grasp, but also to determine its orientation, providing an additional exteroceptive input for the semi-autonomous control. Second, methods for estimating the contextual features from the sensory modalities have to be developed. Inertial units, for example, can be used to detect the movement phases (e.g., start and end of reaching) as well as other features (e.g., movement speed, direction). The present evaluation considered a simple scene including one object at a time. However, even at this early phase of development, the system would be able to handle more complex and cluttered environments, as partly demonstrated with the previous prototype (see figure 10 in [49]). The overall system robustness and performance in a realistic environment is the main scope of these developments. For example, a depth sensor was selected rather than a stereo-camera, the computer vision module 
assumes that the objects are unknown rather than using a predetermined database (limited object set), etc. The scene and user analysis is being continuously improved. We are currently developing the sophisticated SLAM-based [65] approaches in conjunction with advanced clusterization algorithms [66]. This would ultimately result in advanced object modeling schemes capable of simultaneously handling and identifying multiple objects of interest together with their respective features (e.g., object affordances, multiple objects stacked together, etc). Yet another improvement can be implemented in the algorithm for object selection, which is certainly sub-optimal since the user first needs to turn his head towards the object and then trigger the system. Namely, it has been shown that during grasping the subjects mostly move the eyes rather than the head [67]. Therefore, the object selection could be improved by using a practical eye tracking system, embedded into the glasses [68], but such systems are still rather expensive. Another, more feasible and cost effective, solution would be to perform hand tracking algorithm using fusing vision [69, 70] and advanced inertial sensing [71]. This would allow the CASP system to infer the object that is likely to be grasped by the user (e.g., the one towards which the hand moves) thus eliminating the need for explicit head movement.

In the present study, the grasp kinematics was influenced by the specific experimental setup. Therefore, the joint angles were not measured in order to identify normal versus abnormal movements in real life conditions, but mainly to compare how different control systems influence the kinematics in an identical setup. When the CASP system reaches the stage of development in which the components are properly integrated and ergonomically attached to the subjects (e.g., prosthesis on the socket, sensors miniaturized), the kinematics will be acquired and compared to those of able-bodied subjects to assess the naturalness of the movement.

The experimental evaluation performed in this study was designed to test the novel system prototype for its potential transferability and overall applicability in the prosthesis control, and especially how the semi-autonomous control at this stage of development compares to the classic manual operation (commercial and clinical benchmark). Therefore, the study focus was set primarily on performing a set of functionally relevant ad-hoc experiments (e.g., grasping and lifting differently oriented objects) designed for heavy-duty multi-DOF prosthesis usage, taking into account also the constraints of the setup (e.g., vision sensor worn by the users, prosthesis mounted on the splint). The ad-hoc test used in the present study was therefore the result of a compromise and was not psychometrically validated. However, such tests are not unusual in the scientific literature, even when evaluating ready-to-use systems $[22,72]$. We are fully aware of the importance of standardized evaluations and of the current efforts in the scientific community towards establishing such benchmarks [73, 74]. Therefore, in the more mature development phase, as the system approaches its clinical applicability, and amputees are able to utilize it in a practical setup (integrated components), the current test will be substituted by the psychometrically relevant measures (e.g., SHAP [75]).

\section{Acknowledgments}

We acknowledge financial support by the German Ministry for Education and Research (BMBF) via the Bernstein Focus Neurotechnology (BFNT) Göttingen (grant \#1GQ0810) (DF) as well as the EU-funded MYOSENS project (grant\#FP7PEOPLE-2011-IAPP-26208).

\section{References}

[1] MacKenzie C L and Iberall T 1994 Advances in Psychology: The Grasping Hand vol 104 (Amsterdam: North Holland)

[2] Cutkosky M R 1989 On grasp choice, grasp models, and the design of hands for manufacturing tasks IEEE Trans. Robot. Autom. 5 269-79

[3] Churchill A, Hopkins B, Rönnqvist L and Vogt S 2000 Vision of the hand and environmental context in human prehension Exp. Brain Res. 134 81-9

[4] Connolly J D and Goodale M A 1999 The role of visual feedback of hand position in the control of manual prehension Exp. Brain Res. 125 281-6

[5] Ingram H A, van Donkelaar P, Cole J, Vercher J L, Gauthier G M and Miall R C 2000 The role of proprioception and attention in a visuomotor adaptation task Exp. Brain Res. 132 114-26

[6] Ziegler-Graham K, MacKenzie E J, Ephraim P L, Travison T G and Brookmeyer R 2008 Estimating the prevalence of limb loss in the United States: 2005 to 2050 Arch. Phys. Med. Rehabil. 89 422-9

[7] Fougner A, Stavdahl O, Kyberd P J, Losier Y G and Parker P A 2012 Control of upper limb prostheses: terminology and proportional myoelectric control-a review. IEEE Trans. Neural Syst. Rehabil. Eng. 20 663-77

[8] Belter J T, Segil J L, Dollar A M and Weir R F 2013 Mechanical design and performance specifications of anthropomorphic prosthetic hands: A review J. Rehabil. Res. Dev. 50 599-618

[9] Otto Bock HealthCare GmbH, Sensor Hand Speed ${ }^{\circledR}$ (http:// ottobock.de/cps/rde/xchg/ob_com_en/hs.xsl/3652.html)

[10] Otto Bock HealthCare GmbH, Michelangelo ${ }^{\circledR}$ (http:// ottobock.com/cps/rde/xchg/ob_com_en/hs.xsl/ 49464.html)

[11] Touch Bionics iLimb (http://touchbionics.com/products/ active-prostheses /)

[12] DARPA (http://darpa.mil/Our_Work/BTO/Programs/ Revolutionizing_Prosthetics.aspx)

[13] Ning J, Dosen S, Muller K-R and Farina D 2012 Myoelectric control of artificial limbs-is there a need to change focus? IEEE Signal Process. Mag. 29 152-150

[14] Amsuess S, Goebel P, Graimann B and Farina D 2014 Extending mode switching to multiple degrees of freedom in hand prosthesis control is not efficient Proc. Ann. Int. Conf. IEEE Engineering in Medical and Biology Society pp 658-61

[15] Fifer M S et al 2014 Simultaneous neural control of simple reaching and grasping with the modular prosthetic limb using intracranial EEG IEEE Trans. Neural Syst. Rehabil. Eng. 22 695-705

[16] Xiao R and Ding L 2013 Evaluation of EEG features in decoding individual finger movements from one hand Comput. Math. Methods Med. 2013243257 
[17] Suzuki R, Ogino K, Nobuaki K, Kogure K and Tanaka K 2013 Development of meal support system with voice input interface for upper limb disabilities Proc. of the IEEE 8th Conf. on Industrial Electronics and Applications (ICIEA) pp 714-8

[18] Carrozza M C, Persichetti A, Laschi C, Vecchi F, Lazzarini R, Vacalebri P and Dario P 2007 A wearable biomechatronic interface for controlling robots with voluntary foot movements IEEE/ASME Trans. Mechatronics 12 1-11

[19] Coapt (http://coaptengineering.com/)

[20] Baker J J, Scheme E, Englehart K, Hutchinson D T and Greger B 2010 Continuous detection and decoding of dexterous finger flexions with implantable myoelectric sensors IEEE Trans. Neural Syst. Rehabil. Eng. 18 424-32

[21] Kuiken T A, Dumanian G A, Lipschutz R D, Miller L A and Stubblefield K A 2004 The use of targeted muscle reinnervation for improved myoelectric prosthesis control in a bilateral shoulder disarticulation amputee Prosthet. Orthot. Int. 28 245-53

[22] Amsuess S, Gobel P, Graimann B and Farina D 2014 A multiclass proportional myocontrol algorithm for upper limb prosthesis control: validation in real-life scenarios on amputees IEEE Trans. Neural Syst. Rehabil. Eng. 23 827-36

[23] Jiang N, Englehart K B and Parker P A 2009 Extracting simultaneous and proportional neural control information for multiple-DOF prostheses from the surface electromyographic signal IEEE Trans. Biomed. Eng. 56 1070-80

[24] Castellini C, Passig G and Zarka E 2012 Using ultrasound images of the forearm to predict finger positions IEEE Trans. Neural Syst. Rehabil. Eng. 20 788-97

[25] Bodenhagen L, Fugl A R, Jordt A, Willatzen M, Andersen K A, Olsen M M, Koch R, Petersen H G and Kruger N 2014 An adaptable robot vision system performing manipulation actions with flexible objects IEEE Trans. Autom. Sci. Eng. 11 749-65

[26] Chitta S, Jones E, Ciocarlie M and Hsiao K 2012 Mobile manipulation in unstructured environments: perception, planning, and execution IEEE Robot. Autom. Mag. 19 $58-71$

[27] Swain I D and Nightingale J M 1980 An adaptive control system for a complete hand/arm prosthesis J. Biomed. Eng. 2 163-6

[28] Tomovic R and Boni G 1962 An adaptive artificial hand IRE Trans. Autom. Control 7 3-10

[29] Dillon S S 1995 Automatic control of wrist rotation in artificial arm Proc. of the MyoElectric Controls Prosthetics Symp. pp 110-1

[30] Banziger E 1996 Wrist rotation activation in myoelectric prosthetics - an innovative approach: a case study $A C P O C$ News 30 12-4

[31] Nightingale J M 1985 Microprocessor control of an artificial arm J. Microcomput. Appl. 8 167-73

[32] Chappell P H, Nightingale J M, Kyberd P J and Barkhordar M 1987 Control of a single degree of freedom artificial hand J. Biomed. Eng. $9273-7$

[33] Tomovic R, Bekey G and Karplus W 1987 A strategy for grasp synthesis with multifingered robot hands Proc. of the IEEE Int. Conf. on Robotics and Automation vol 4 pp 83-9

[34] Wettels N, Parnandi A R, Loeb G E and Sukhatme G S 2009 Grip control using biomimetic tactile sensing systems IEEE/ ASME Trans. Mechatronics 14 718-23

[35] Light C M, Chappell P H, Hudgins B and Engelhart K 2002 Intelligent multifunction myoelectric control of hand prostheses J. Med. Eng. Technol. 26 139-46

[36] Goto S, Nakamura M and Sugi T 2008 Development of meal assistance orthosis for disabled persons using EOG signal and dish image Int. J. Adv. Mechatron. Syst. 1107
[37] Novak D and Riener R 2013 Enhancing patient freedom in rehabilitation robotics using gaze-based intention detection Proc. of the IEEE Int. Conf. on Rehabilitation Robotics

[38] Muelling K, Venkatraman A, Valois J-S, Downey J, Weiss J, Javdani S, Hebert M, Schwartz A B, Collinger J L and Bagnell J A 2015 Autonomy infused teleoperation with application to BCI manipulation (arXiv:1503.05451)

[39] Controzzi M, Cipriani C, Popovic D B and Carrozza M C 2013 Controlling hand-assistive devices: utilizing electrooculography as a substitute for vision IEEE Robot. Autom. Mag. 20 40-52

[40] Novak D and Riener R 2014 A survey of sensor fusion methods in wearable robotics Rob. Auton. Syst. 73 $155-70$

[41] McMullen D et al 2014 Demonstration of a semi-autonomous hybrid brain-machine interface using human intracranial EEG, eye tracking, and computer vision to control a robotic upper limb prosthetic IEEE Trans. Neural Syst. Rehabil. Eng. 22 784-96

[42] Johnson M, Bradshaw J M, Feltovich P J, Jonker C M, Van Riemsdijk M B and Sierhuis M 2014 Coactive design: designing support for interdependence in joint activity J. Human-Robot Interact. 343

[43] EUrobotics 2015 Robotics 2020 Multi-Annual Roadmap pp 178-228

[44] Muzumdar A (ed) 2004 Powered Upper Limb Prostheses (Berlin: Springer) pp 35-54

[45] Creative Technology Ltd, Senz3D ${ }^{\circledR}$ (http://de.creative.com/ $\mathrm{p} /$ web-cameras /creative-senz3d)

[46] Dosen S, Markovic M, Popović D B, Farina D and Graimann B 2013 Sensor fusion for control of upper limb protheses: integration of myoelectric control with stereovision, augmented reality and inertial sensing EP 13171671.4

[47] Microsoft Corporation, Microsoft HoloLens ${ }^{\circledR}$ (https:// microsoft.com/microsoft-hololens/en-us)

[48] Meta Inc., Meta 1 developer kit (https://getameta.com/)

[49] Markovic M, Dosen S, Cipriani C, Popovic D and Farina D 2014 Stereovision and augmented reality for closed-loop control of grasping in hand prostheses J. Neural Eng. 11 046001

[50] Dosen S, Cipriani C, Kostić M, Controzzi M, Carrozza M C and Popović D B 2010 Cognitive vision system for control of dexterous prosthetic hands: experimental evaluation J. Neuroeng. Rehabil. 742

[51] Matija Š, Kočović S, Marković M and Popović D B 2014 Microsoft kinect-based artificial perception system for control of functional electrical stimulation assisted grasping Biomed. Res. Int. 201412

[52] Marković M, Graimann B, Došen S and Farina D 2014 Computer vision for automatic control of orientation and preshape in a hand prosthesis with an active wrist MEC2014 pp 143-5

[53] Luo R C, Chang C C and Lai C C 2011 Multisensor fusion and integration: theories, applications, and its perspectives IEEE Sens. J. 11 3122-38

[54] Pugh A 1991 The robot story Comput. Contr. Eng. J. 2 13-21

[55] Smith L H, Kuiken T A and Hargrove L J 2014 Real-time simultaneous and proportional myoelectric control using intramuscular EMG J. Neural Eng. 11066013

[56] Kermorgant O and Chaumette F 2011 Multi-sensor data fusion in sensor-based control: application to multi-camera visual servoing Proc. of the IEEE Int. Conf. on Robotics and Automation pp 4518-23

[57] Jones L E and Davidson J H 1999 Save that arm: a study of problems in the remaining arm of unilateral upper limb amputees Prosthet. Orthot. Int. 23 55-8 
[58] Hart S G and Staveland L E 1988 Development of NASA-TLX (Task Load Index): Results of Empirical and Theoretical Research (Amsterdam: Elsevier) vol 52

[59] Gonzalez J, Soma H, Sekine M and Yu W 2012 Psychophysiological assessment of a prosthetic hand sensory feedback system based on an auditory display: a preliminary study J. Neuroeng. Rehabil. 933

[60] Touch Bionics, Inc., Grip chips (http://touchbionics.com/ sites/default/files/files/Grip Chip datasheet May 2014.pdf)

[61] IBT, morph (http://i-biomed.com/morph.html)

[62] Vilarino M 2014 Technical Note: A Novel Wireless Controller for Switching among Modes for an Upper-Limb Prosthesis (www.oandp.org/AcademyTODAY/2014Jan/4.asp)

[63] Vilarino M, Moon J, Rogner K, Varghese J, Ryan T, Thakor N V and Kaliki R 2015 Outcomes and perception of a conventional and alternative myoelectric control strategy: a study of experienced and new multiarticulating hand users Am. Acad. Orthot. Prosthetics 7 53-62

[64] Krigslund R, Dosen S, Popovski P, Dideriksen J L, Pedersen G F and Farina D 2013 A novel technology for motion capture using passive UHF RFID tags IEEE Trans. Biomed. Eng. 60 1453-7

[65] Aulinas J, Petillot Y, Salvi J and Lladó X 2008 The SLAM problem: a survey in Frontiers Artif. Intell. Appl. 184 363-71

[66] Stein S C, Worgotter F, Schoeler M, Papon J and Kulvicius T 2014 Convexity based object partitioning for robot applications Proc. of the IEEE Int. Conf. on Robotics and Automation (ICRA) pp 3213-20
[67] Shumway-Cook A and Woollacott M H 2007 Motor Control: Translating Research Into Clinical Practice (Philadelphia, PA: Lippincott Williams and Williams)

[68] SensoMotoric Instruments Inc., SMI Eye Tracking Glasses ${ }^{\circledR}$ (http://eyetracking-glasses.com/)

[69] Intel Corporation, RealSense ${ }^{\mathrm{TM}}$ SDK (https://software.intel. com/en-us/intel-realsense-sdk/details)

[70] Sun L, Liu G and Liu Y 2014 3D hand tracking with head mounted gaze-directed camera IEEE Sens. J. 14 1380-90

[71] El-Gohary M and McNames J 2015 Human joint angle estimation with inertial sensors and validation with a robot arm IEEE Trans. Biomed. Eng. 62 1759-67

[72] Cipriani C, Zaccone F, Micera S and Carrozza M C 2008 On the shared control of an EMG-controlled prosthetic hand: analysis of user-prosthesis interaction IEEE Trans. Robot. 24 170-84

[73] Hill W, Kyberd P, Hermanssom L, Hubbarad S, Stavdahl O, Swanson S and Cooper M 2009 Upper limb prosthetic outcome measures (UPLOM): a working group and their findings J. Prosthetics Orthot. 21 69-82

[74] Hill W, Stavdahl Ø, Liselotte H, Kyberd P, Swanson S and Hubbarad S 2009 'Functional outcomes in the WHOICF model: establishment of the upper limb prosthetic outcome measures group J. Prosthetics Orthot. 21 115-9

[75] Light C M, Chappell P H and Kyberd P J 2002 Establishing a standardized clinical assessment tool of pathologic and prosthetic hand function: normative data, reliability, and validity Arch. Phys. Med. Rehabil. 83 776-83 


\section{APPENDIX 3: SENSORY FEEDBACK IN PROSTHETICS: A STANDARDIZED TEST BENCH FOR CLOSED-LOOP CONTROL}


Sensor Fusion for Closed-loop Control of Upper-limb Prostheses 


\title{
Sensory Feedback in Prosthetics: A Standardized Test Bench for Closed-Loop Control
}

\author{
Strahinja Dosen, Member, IEEE, Marko Markovic, Cornelia Hartmann, Student Member, IEEE, and \\ Dario Farina, Senior Member, IEEE
}

\begin{abstract}
Closing the control loop by providing sensory feedback to the user of a prosthesis is an important challenge, with major impact on the future of prosthetics. Developing and comparing closed-loop systems is a difficult task, since there are many different methods and technologies that can be used to implement each component of the system. Here, we present a test bench developed in Matlab Simulink for configuring and testing the closed-loop human control system in standardized settings. The framework comprises a set of connected generic blocks with normalized inputs and outputs, which can be customized by selecting specific implementations from a library of predefined components. The framework is modular and extensible and it can be used to configure, compare and test different closed-loop system prototypes, thereby guiding the development towards an optimal system configuration. The use of the test bench was demonstrated by investigating two important aspects of closed-loop control: performance of different electrotactile feedback interfaces (spatial versus intensity coding) during a pendulum stabilization task and feedforward methods (joystick versus myocontrol) for force control. The first experiment demonstrated that in the case of trained subjects the intensity coding might be superior to spatial coding. In the second experiment, the control of force was rather poor even with a stable and precise control interface (joystick), demonstrating that inherent characteristics of the prosthesis can be an important limiting factor when considering the overall effectiveness of the closed-loop control. The presented test bench is an important instrument for investigating different aspects of human manual control with sensory feedback.
\end{abstract}

Index Terms-Closed-loop prosthetic system, electrocutaneous stimulation, electrotactile stimulation, sensory feedback, vibrotactile stimulation.

\section{INTRODUCTION}

$\mathbf{U}$ PPER extremity prostheses are devices that aim to replace, morphologically and functionally, the hand and a part of the arm or even the whole limb that is lost due to an amputation. The flexible musculoskeletal structure of

Manuscript received March 14, 2014; revised September 14, 2014; accepted October 25, 2014. Date of publication November 20, 2014; date of current version March 05, 2015. This work was supported by the European Commission under the MYOSENS (FP7-PEOPLE-2011-IAPP-286208) Project and by the German Ministry for Education and Research (BMBF) via the Bernstein Focus Neurotechnology (BFNT) Göttingen under Grant 01GQ0810.

The authors are with the Department of Neurorehabilitation Engineering, University Medical Center Göttingen, Georg-August University, Göttingen, Germany (e-mail: strahinja.dosen@bccn.uni-goettingen.de; marko.markovic@bccn.uni-goettingen.de; cornelia.hartmann@bccn.unigoettingen.de; dario.farina@bccn.uni-goettingen.de).

Color versions of one or more of the figures in this paper are available online at http://ieeexplore.ieee.org.

Digital Object Identifier 10.1109/TNSRE.2014.2371238 the human arm allows for dexterous reaching, grasping and manipulation, but a smooth, effortless, and highly adaptive execution of these movements would not be possible without a network of sensors providing proprioceptive and exteroceptive information to the brain. In an ideal case, an artificial replacement of the hand/arm should restore both motor and sensory functions by implementing a bidirectional communication between the system and the user's brain. However, there are still no commercially available devices implementing any kind of (intentional) somatosensory feedback to the user.

A specific challenge when developing a closed-loop prosthetic system is that there are many potential methods and technologies, and in order to select an optimal configuration based on objective criteria, different systems should be implemented, tested and compared. The general components comprising the control loop are well defined: the input interface providing command signals, the system to be controlled, and the stimulation interface transmitting feedback information to the user. However, in reality, many different technologies are available to implement each of these components and there are multiple internal parameters that need to be adjusted. For example, a myoelectric prosthesis can be controlled using surface [1] or intramuscular [2] electromyography (EMG) with a varying number of input channels, from a conventional two-channel interface to a high density EMG [1], [3]. Moreover, commercially available hand prostheses have very different mechanical characteristics; they can be simple open/close grippers (e.g., Sensor Hand Speed from Otto Bock [4]), more flexible systems implementing two grasp types (Michelangelo Hand from Otto Bock [4]), or dexterous mechanisms with individually controllable fingers (Bebionic Hand from RSLSteeper [5], i-Limb from Touch Bionics [6]). The feedback information can be transmitted through different sensory modalities, using the method of sensory substitution [7]. The tactile sense for example can be stimulated by using miniature mechanical actuators (e.g., vibration motors, linear pushers) or electrical currents activating cutaneous afferents [8]. In both cases, the stimulation can be delivered using different coding schemes, i.e., through single or multiple stimulation units by modulating stimulation parameters and/or active channel(s) [8]. Finally, in some investigations it may be useful to simulate virtual dynamic systems (e.g., inverted pendulum [9], prosthesis models [10]) instead of real prostheses in order to evaluate and compare the feedback methods.

Many studies have investigated sensory feedback and closedloop control in prosthetics [11]. In most cases, a specific configuration of the closed-loop system was selected based on the individual preferences/heuristics of the authors and/or hardware 

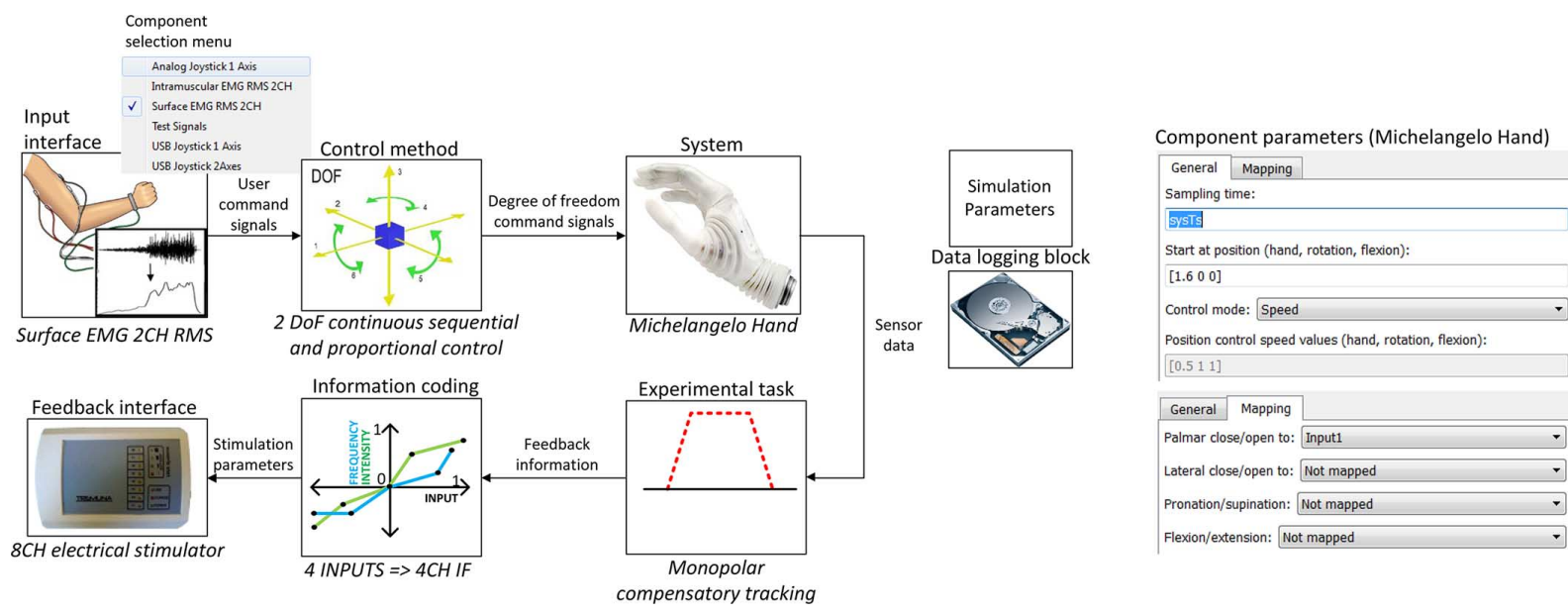

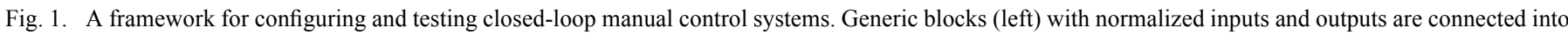

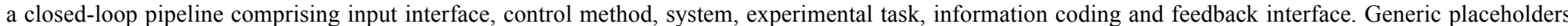

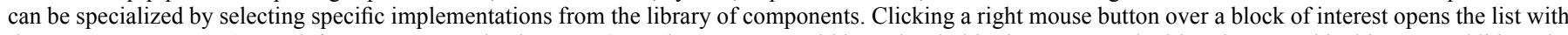

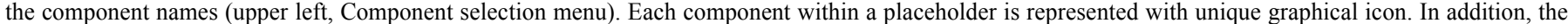

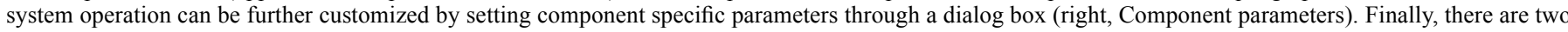
accessory blocks with global scope, i.e., general simulation parameters and data logging setup (middle blocks).

availability, and the performance was then evaluated. To this aim, different tests have been used: lifting real objects [12], handling specially designed test samples [13], generating target forces or angles [14], etc. Due to different setups and tests, it is very difficult to compare the results of the studies. Finally, there are very few studies directly comparing different closed-loop systems under the same conditions [15].

In this paper, we present a flexible test bench for testing the human manual control with sensory feedback. The test bench is modular and extensible and operates in real time. It allows fast and easy setup of specific closed-loop system configurations through a simple selection of the desired components from predefined libraries. By utilizing the test bench we aim to achieve an objective, standardized (i.e., same conditions and tasks), and direct comparison of different solutions for the closed-loop control of prosthetic devices. In the following, we describe the system and illustrate its application by means of two pilot experiments. The framework was used to address specific and relevant aspects related to both feedback and feedforward components of a closed-loop system.

\section{MethodS}

\section{A. Closed-Loop Test Bench}

The test bench was implemented using Matlab 2013a (MathWorks, US), Simulink, Simulink 3D Animation (3DANI) and Real Time Windows Target toolbox (RTWT). Using Simulink, the system was built by connecting functional blocks, while RTWT allowed real time execution of the Simulink model. 3DANI was used for visualization.

The test bench is depicted in Fig. 1. It comprises a set of generic blocks (placeholders) connected into a prototype closed-loop system. The connections between the blocks represent the flow of multidimensional signals, where all the signals are normalized to intervals $[0,1]$ or $[-1,1]$, and have well-defined semantics. This generic closed-loop system can be customized into a number of specific configurations by selecting a particular implementation for each of the generic blocks. To this aim, each block corresponds to the library of components (Fig. 2), and the currently active implementation can be selected by choosing from the list of component names. The components are also represented by an intuitive graphical icon. Importantly, since the blocks share a common interface (i.e., normalized, well-defined signals), all the components from the libraries are interoperable and can be therefore combined with virtually no constraints. Thus, any configuration of components selected by the user comprises a valid system that can be executed and tested. Once the components are selected, the system can be further customized by adjusting the internal parameters of each component (Fig. 1).

The input interface block implements the generation, acquisition and preprocessing of the signals from different information sources (e.g., muscle activity, joystick, data file). The block outputs monopolar signals, which can be commands generated by the user (e.g., when using a joystick or myoelectric control) or predefined signals read from the file implementing specific tests/scenarios. In this context, each signal represents a normalized high-level (user) command, whose exact semantics is determined by the subsequent blocks in the loop (e.g., controlled system). Some of the components that are currently implemented in the library are: multichannel surface EMG, multichannel intramuscular EMG, single axis analog joystick, two axes USB joystick, load cell, file input, etc. As explained, all input interfaces share a common structure: each channel of EMG outputs one monopolar normalized signal, whereas a joystick axis provides two, i.e., one for the movement of the handle in each direction (e.g., left and right inclination). The joystick button triggers the co-activation of both channels, which is equivalent to a co-contraction in myocontrol. The blocks also implement preprocessing (e.g., baseline correction, dead zone, normalization, filtering etc.) 
The control method block implements the mapping from the user-level to the system-level commands. This is an arbitrary transformation of a set of monopolar input signals into a set of bipolar output signals. The latter are meant to drive active degrees of freedom (DoFs) of the controlled system in both directions. Currently, there are components in the library implementing continuous and discrete proportional control of single and multiple DoFs, as well as simple state-based methods. For example, 2 DoF continuous sequential and proportional control block subtracts two monopolar inputs (e.g., two EMG channels) to obtain a bipolar command and also detects the co-activation at the two channels to switch the bipolar signal between the two outputs. In other blocks, signals can be discretized into a set of predefined levels (discrete sequential and proportional control block) or used to trigger state transitions (state machine control block).

The system block encapsulates a low-level interface to a real or simulated system that is controlled in the closed loop. The block defines intrinsic system parameters and also the mapping between the input control signals and the available system DoFs, defining which signal commands which system function. The outputs from the block are monopolar signals delivering the sensor data assessing the current state of the system, such as, position and/or exerted (interaction) force. The components currently available in the library are inverted pendulum model, Michelangelo hand (4 DoF), virtual hand model (3 DoF) and a model of an arbitrary 2 DoF system. For example, using the prosthesis block, the user can define an initial configuration (finger and wrist positions), command mode (absolute position or speed control) and also connect, in an arbitrary order, the control signals coming from a selected input interface (e.g., EMG or joystick) to the four available active DoFs (palmar grasp open/close, lateral grasp open/close, wrist pronation/supination, and wrist flexion/extension). As an illustration, a single axis joystick can be combined with a 1 DoF continuous proportional control block and set to control either a prosthesis open/close or pronation/supination individually (i.e., dedicated single DoF tests). Alternatively, the same input interface can be used with a 2 DoF continuous sequential and proportional control block to operate proportionally both of those DoFs using co-activation to switch between the two (sequential control).

The experimental task block compares the current versus desired system state, as defined by sensor data (system block) and the goal of the experiment, respectively. Based on this error signal, it determines the signals that should be fed back to the user. Currently, monopolar and bipolar compensatory and pursuit tracking as well as reach the target window protocols are implemented in the library. For example, if the compensatory tracking task is selected, the goal for the subject is to null (compensate) the tracking error. Therefore, the block subtracts the current system state from the predefined reference trajectory to compute the momentary tracking error (e.g., a difference between the current and desired grasping force). This is the feedback signal to be transmitted to the user.

The information coding block translates the normalized feedback information signals into the normalized stimulation parameters (i.e., frequency and intensity) for a generic multichannel feedback device. Different generic information coding schemes can be defined and saved, e.g., single and multichannel spatial coding and intensity modulations. The input feedback information signals are then simply "connected" to the desired mappings to implement particular feedback configurations. For example, a prosthesis aperture can be fed back using an array of electrodes (spatial coding), while the grasping force could be transmitted using a single channel intensity or frequency modulation. Currently, there is a range of predefined mappings defined in the library using lookup tables. Also, a simple user interface is provided to create new lookups.

Finally, the feedback interface block encapsulates a low-level communication with a device that is used to deliver the feedback. The block sends device specific commands to adjust the stimulation parameters to the normalized frequency and intensity values supplied by the information coding block. Currently, the supported devices are multichannel electrical stimulators with concentric and matrix electrodes, vibration motors [16], and several forms of visual feedback (e.g., virtual stimulator, signal scope, object tracking and virtual hand). In the case of vibration motors, the translation of normalized parameters is straightforward: they are mapped into intensity and frequency of the generated vibrations. When using electrical stimulation, the user can select if the intensity should be implemented through pulse width or amplitude modulations.

\section{B. Experimental Evaluation}

To demonstrate the working principles and potential applications, we present two pilot experiments conducted using the developed framework. In the first experiment, several feedback configurations were evaluated by controlling a simulated system. In the second, two feedforward interfaces were compared while operating a real prosthesis.

1) Experiment 1: Comparing Feedback Interfaces: In this test, the generic blocks were configured as follows: analog joystick single axis $=>1$ DoF continuous proportional control $=>$ inverted pendulum model $=>$ information coding $[12 \mathrm{CH}-\mathrm{S}$, $8 \mathrm{CH}-\mathrm{SI}, 8 \mathrm{CH}-\mathrm{I}, 2 \mathrm{CH}-\mathrm{I}]=>2 \times$ electrotactile stimulator. Note that several components were used for the information coding block corresponding to the feedback interfaces that were tested (annotation: $\mathrm{CH}$ - number of channels, $\mathrm{S}$ - spatial and I-intensity coding). The task for the subject was to stabilize a simulated planar inverted pendulum using an analog joystick, while the feedback about the pendulum inclination was provided via electrotactile stimulation. The joystick inclination was proportional to the torque at the base of the pendulum. To deliver the electrotactile feedback, 12 concentric electrodes (CoDe 501500 $\varnothing 40 \mathrm{~mm}$, SpesMedica, IT) were positioned along the dorsal and volar side of the forearm, six electrodes on each side. The stimulation was provided by using multichannel stimulation unit (RehaStim, Hasomed Gmbh, DE).

The side of the forearm (dorsal, volar) to which the stimulation was delivered denoted the side toward which the pendulum was inclined (left, right), and the amount of inclination was coded using spatial and/or intensity coding. The following configurations were tested:

- Spatial coding with $2 \times 6$ channels $(12 \mathrm{CH}-\mathrm{S})$. The $n$th electrode was activated if the inclination angle was within 
the interval $[(n-1) \pi / 12, n \pi / 12], n=1,2 \ldots 6$, otherwise it was off (i.e., only one electrode active at a time).

- Combined spatial and intensity coding with $2 \times 4$ channels (8CH-SI). The $n$th electrode was activated if the inclination angle was within the interval $[(n-1) \pi / 8, n \pi / 8], n=$ $1,2,3,4$. In addition, the stimulation intensity of the currently active electrode was modulated, linearly mapping the corresponding interval of inclinations to the entire dynamic range of the electrode.

- Intensity coding with $2 \times 4$ channels $(8 \mathrm{CH}-\mathrm{I})$. The stimulation intensity was modulated proportionally to the inclination angle on all four electrodes simultaneously, linearly mapping the full interval of inclinations $[0, \pi / 2]$ to the entire dynamic range of each electrode.

- Intensity coding with $2 \times 1$ channel $(2 \mathrm{CH}-\mathrm{I})$. The same as $8 \mathrm{CH}-\mathrm{I}$, but using only one electrode at each side of the forearm.

For the spatial coding (12CH-S), the stimulation at each electrode was constant and equal to $\mathrm{ST}+0.5 \cdot \mathrm{R}$, with $\mathrm{R}=(\mathrm{PT}-$ $\mathrm{ST}$ ), where ST and PT are the sensation and pain thresholds, respectively. For the intensity coding, the dynamic range was determined as $[\mathrm{ST}+0.1 \cdot \mathrm{R}, \mathrm{ST}+0.8 \cdot \mathrm{R}]$. The ST and PT were estimated for each electrode using the method of limits [17]. Pulsewidth modulation was employed to regulate the stimulation intensity, while the current amplitude was set at $3 \mathrm{~mA}$ and frequency at $100 \mathrm{~Hz}$. This setup was used since it resulted in well perceived, discriminable sensations.

Six subjects signed a consent form and participated in the experiment ( $30 \pm 5$ years), which was approved by the local ethic committee. The experiment included two sessions performed on two consecutive days. During the first introductory session, which lasted 30 to $45 \mathrm{~min}$, the subjects were introduced to the task. They stabilized the pendulum using visual feedback (planar pendulum), simultaneous visual and electrotactile feedback (2CH-I) and only electrotactile feedback. In the main testing session, the subjects performed the pendulum stabilization tasks using feedback interfaces in the following order: $12 \mathrm{CH}-\mathrm{S}, 8 \mathrm{CH}-\mathrm{SI}, 8 \mathrm{CH}-\mathrm{I}$ and $2 \mathrm{CH}-\mathrm{I}$. The subjects started with the most intuitive interface (simple spatial coding) and finished with the most difficult one (intensity coding, two electrodes only). Intensity coding is more demanding for the subjects, since he/she has to discriminate a continuously changing sensation, but it also implements a finer resolution of information transmission. Our pilot tests indicated that the selected challenging control task (i.e., stabilizing an unstable system) would be very difficult if not impossible for the subjects if they would start with the intensity coding interface. The aim of the current experiment was to test if the aforementioned gradual transition between the feedback interfaces would allow the subjects to eventually accomplish the task and even exploit the inherent advantage of the intensity coding. Eleven 90-s long trials were performed in each condition. When the pendulum fell (inclination angle $>=\pi / 2$ ), a short beep was generated, the pendulum position reset (inclination zero), a short destabilizing impulse was delivered to the pendulum, and the trial continued. The pendulum parameters were adjusted so that it reproduced the dynamics of the falling human body rotating around the ankles [18].
2) Experiment 2: Comparing Feedforward Interfaces: In this test, the generic blocks were configured as follows: input interface [analog joystick single axis, surface EMG 2 channels] $=>$ 1 DoF continuous proportional control $=>$ Michelangelo hand $=>$ Monopolar pursuit tracking $=>$ Virtual scope. Note that two components were used for the input interface block corresponding to the two feedforward command interfaces that were compared. The task was to control a prosthetic hand using visual feedback. Specifically, the subjects had to reproduce a reference trapezoidal profile of grasping force: increase the force from the baseline to a predefined level ( $\left.F_{\text {PLATEAU }}\right)$ at a constant rate $\left(F_{\text {Plateau }} / 10 \mathrm{~s}\right)$, stay for $10 \mathrm{~s}$ at the plateau $\left(F_{\text {PlateaU }}\right)$, and then decrease the force back to the baseline at a constant rate $\left(-F_{\text {PLATEAU }} / 10 \mathrm{~s}\right)$. This task emulates the following real-life scenario: the user of a prosthesis grasps an object, slowly increases the force to a target level, holds the object, and then slowly decreases the force to release the object. Three levels were used for $F_{\text {PLATEAU }}$, i.e., $30 \%, 50 \%$ and $70 \%$ of the maximum force the prosthesis can generate $(\sim 110 \mathrm{~N})$. Visual force feedback was provided to the subjects using a graphical scope window plotting the reference and generated force profiles (virtual scope block).

A joystick was used as the benchmark command interface, since it allows precise and stable control signal generation. Myoelectric interface was employed because it is a standard control method for commercially available prostheses. The prosthesis was operated in the conventional velocity mode. Namely, the joystick inclination (left/right) or the level of muscle activity (wrist and finger flexors/extensors) was proportional to the hand velocity (closing/opening) and grasping force (increasing/decreasing). For myocontrol, two channels of bipolar EMG were used (AnEMG12, OTBioelettronica, IT). The EMG was sampled at $1 \mathrm{kHz}$ and processed by calculating the root mean square within a 150 ms sliding window with $90 \%$ overlap.

Five subjects signed a consent form and participated in the experiment ( $28 \pm 6$ years), which was approved by the local ethic committee. Before placing disposable $\mathrm{Ag} / \mathrm{AgCl}$ electrodes (Ambu Neuroline 720), the skin was prepared by applying a small amount of abrasive gel. The order of feedforward interfaces and target force levels was randomized between subjects. The subjects performed ten tracking trials in each condition (feedforward method $\times$ target force level).

3) Data Analysis: For the pendulum test, the number of falls and the average absolute deviation of the pendulum from vertical were computed over each trial. The deviation was expressed in percent of the maximum $(\pi / 2)$. Total energy of the control signal was computed as the surface below the rectified control signal normalized by $C_{\mathrm{MAX}} \cdot T$, where $C_{\mathrm{MAX}}$ is the maximal possible control value $(=1)$ and $T$ is the trial duration. Average time between two successive falls of the pendulum was determined to compare the performance of $2 \mathrm{CH}-\mathrm{I}$ in introductory versus test session. In the force control test, the performance index was the root mean square (RMS) of the tracking error, evaluating the difference between the reference and generated force profiles. RMS was computed for the whole profile as well as over the subsegments of the trajectory, i.e., positive and negative slopes corresponding to force increase and decrease, respectively. When computing 

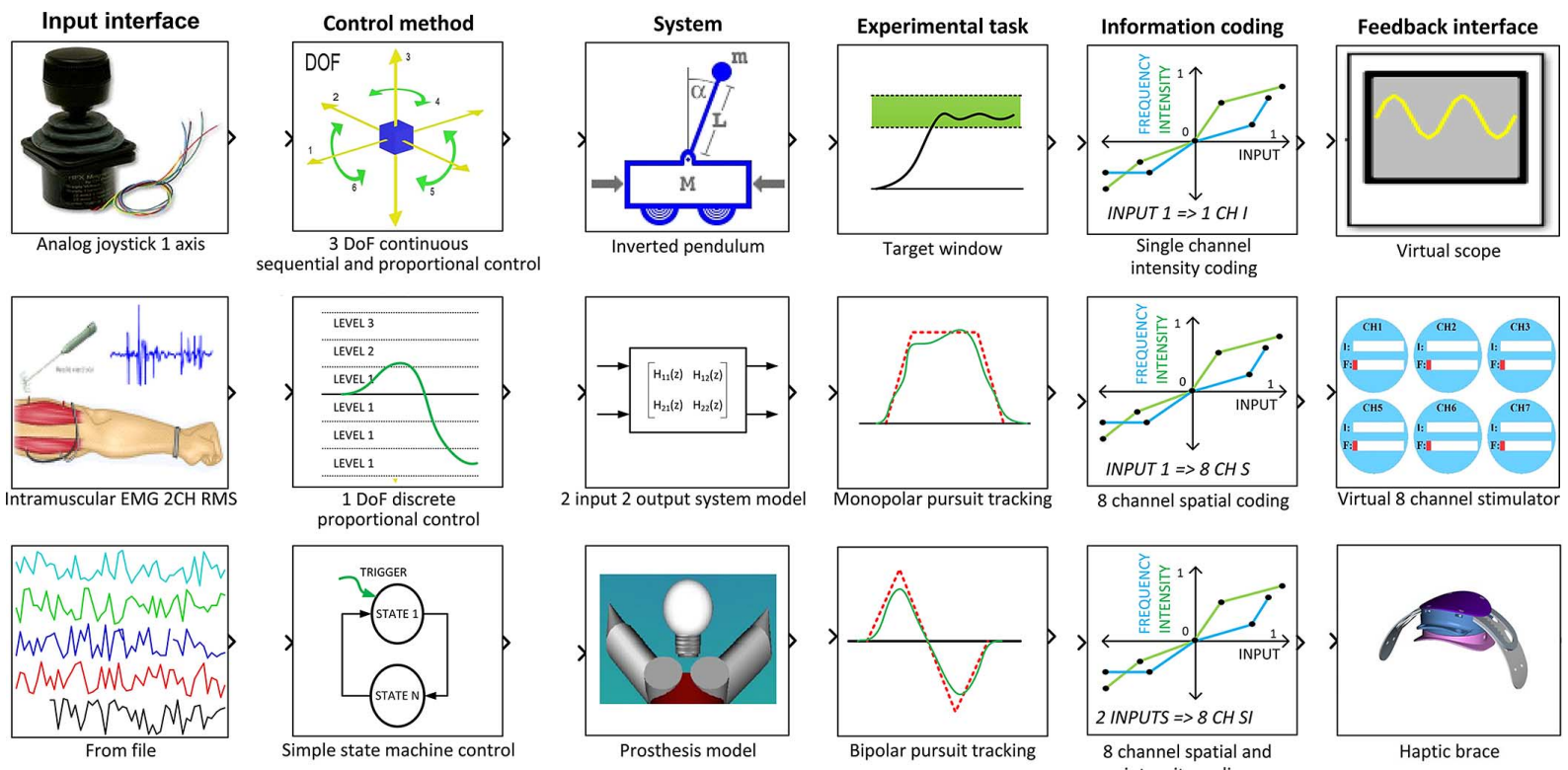

Fig. 2. Several examples from the library of the available components. Column name corresponds to the component type, i.e., the name of the generic block that can be customized by a specific component. Each component is represented by a name and an icon. Some components are sources (input interface), some sinks (feedback interface), and most have both inputs and outputs. Information coding block has the same icon (lookup table mapping) but different lookups were predefined and saved to implement different feedback configurations as suggested by the block annotation.

a)
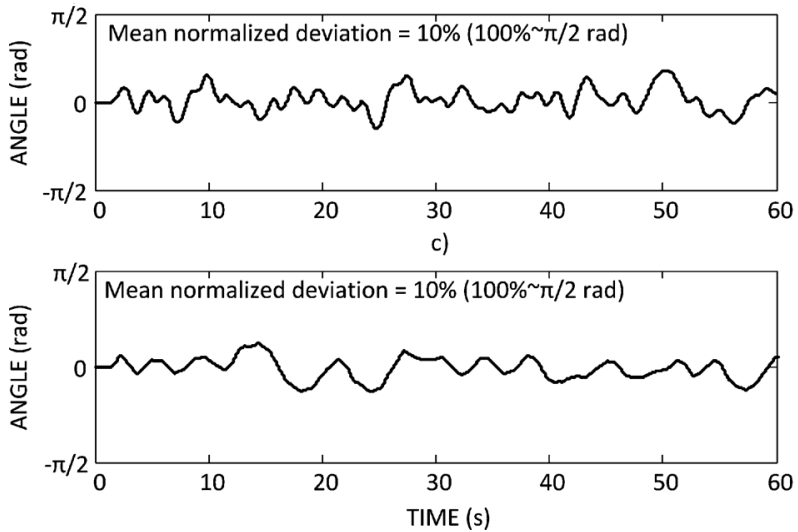

b)
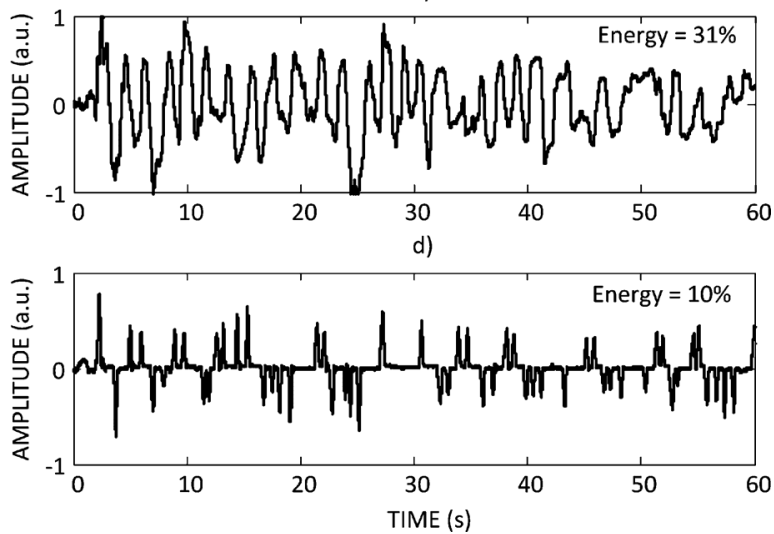

Fig. 3. Example trials of pendulum stabilization using joystick with visual VIS, [a] and [b] and electrotactile feedback 8CH-I, [c] and [d]. Plots (b) and (d) show the control input (joystick movement) and the plots (a) and (c) depict the pendulum inclination. Note that the performance was similar with both feedback types, but the control strategy was very different. With electrotactile feedback, the subjects controlled impulsively. (a) Pendulum angle (VIS), (b) Joystick signal (VIS), (c) Pendulum angle (8CH-I), (d) Joystick signal (8CH-I). The abbreviation $8 \mathrm{CH}-\mathrm{I}$ denotes electrotactile feedback using eight electrodes and intensity coding.

the average performance for both pendulum stabilization and force tracking task, the first five trials in each condition were regarded as training, and they were not used in the calculation. The data analysis and statistical comparison between different experimental conditions was performed in Matlab 2013a (MathWorks, US). We applied Friedman ANOVA and a post hoc test (Tukey's honestly significant difference) for multiple comparisons. Threshold was set to $p<0.05$. To compare two specific conditions, we used Wilcoxon signed-rank test. Numerical results are reported in the text as mean \pm standard deviation.

\section{RESUlts}

1) Comparing Feedback Interfaces: Two example trials of the pendulum stabilization recorded from the same subject using visual (introductory session) and electrotactile (test session) feedback are shown in Fig. 3. The subject successfully stabilized the pendulum for $60 \mathrm{~s}$ with small deviations from the vertical. The performance was similar between the feedback types, but interestingly, the control strategy was very different. When visual feedback was available, the subject continuously modulated the control input (joystick inclination). With electrotactile feedback, however, the control was intermittent, i.e., the subject generated short impulses separated by the intervals of zero control action. Such intermittent control was used consistently by all subjects in electrotactile conditions. The transition from the continuous to intermittent control when switching from visual to electrotactile feedback was observed in four subjects. In the two remaining subjects the transition was not as clear, since they tended to control impulsively even with the visual feedback. The change in the nature of control was reflected by the average normalized energy of the control 
a)
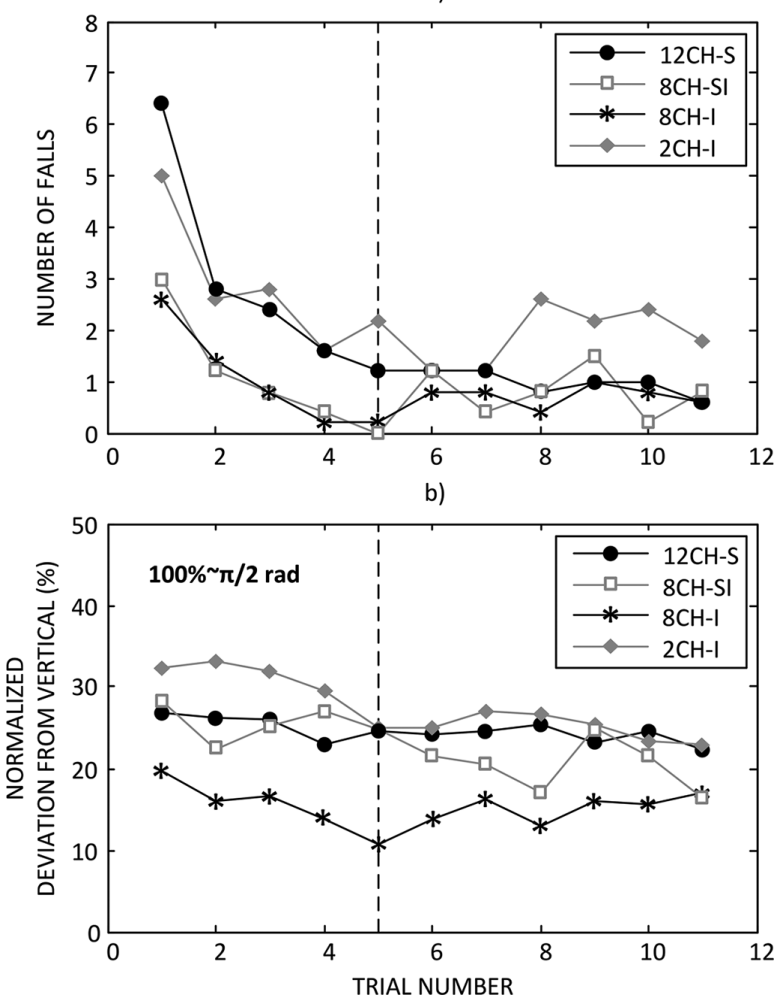

Fig. 4. Results of the pendulum stabilization task across trials: (a) average number of falls (inclination $>\pi / 2$ ), and (b) average deviation from vertical. There was a trend for performance improvement in the first few trials, especially in terms of decreasing the number of falls. After the fifth trial, the performance was more stable. Note that only the mean values are given to reduce the clutter. Each line corresponds to a different feedback interface $(12 \mathrm{CH}$ $8 \mathrm{CH}$ and $2 \mathrm{CH}$ - the number of channels, $\mathrm{S}$ and I-spatial and intensity coding). (a) Average number of falls across trials, (b) Average deviation across trials.

signal, which decreased from $30 \% \pm 11 \%$ for the visual to $20 \% \pm 10 \%$ (12CH-S), $17 \% \pm 11 \%$ (8CH-SI, $p<0.05)$, $16 \% \pm 9 \%(8 \mathrm{CH}-\mathrm{I}, p<0.05)$, and $23 \% \pm 11 \%(2 \mathrm{CH}-\mathrm{I})$ for the electrotactile conditions.

The summary results for the pendulum stabilization task using four electrotactile feedback interfaces are given in Figs. 4 and 5. The subject performance showed a trend of improvement in the first few trials (Fig. 4), with a faster drop in the number of falls [Fig. 4(a)]. However, after the fifth trial, the performance became more stable. Overall, the best and the worst results were obtained using the intensity coding, i.e., 8CH-I and 2CH-I, respectively [Fig. 5(a) and (b)]. More specifically, in terms of keeping the pendulum dynamically stable, the interfaces $12 \mathrm{CH}-\mathrm{S}, 8 \mathrm{CH}-\mathrm{SI}$ and $8 \mathrm{CH}-\mathrm{I}$ resulted in a similar performance, and they were all superior to 2CH-I [Fig. 5(b)]. Although the average deviations were similar for $12 \mathrm{CH}-\mathrm{S}$ and 2CH-I [Fig. 5(a)], the spatial interface still resulted in significantly less falls per trial [Fig. 5(b)]. The interface 8CH-I led to the most precise control [Fig. 5(a)], i.e., the subjects were most successful in minimizing the pendulum oscillations when using this feedback configuration. Also, this configuration was characterized with significantly lower energy of the control signal compared to $12 \mathrm{CH}-\mathrm{S}$ and $2 \mathrm{CH}-\mathrm{I}$. The subject control actions were therefore better timed and/or graded. a)

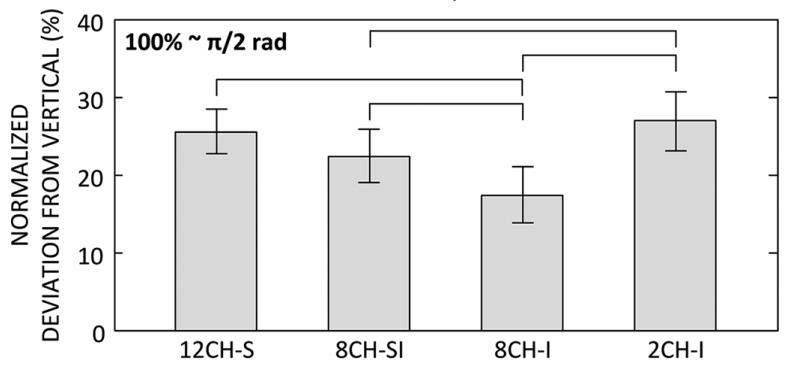

b)

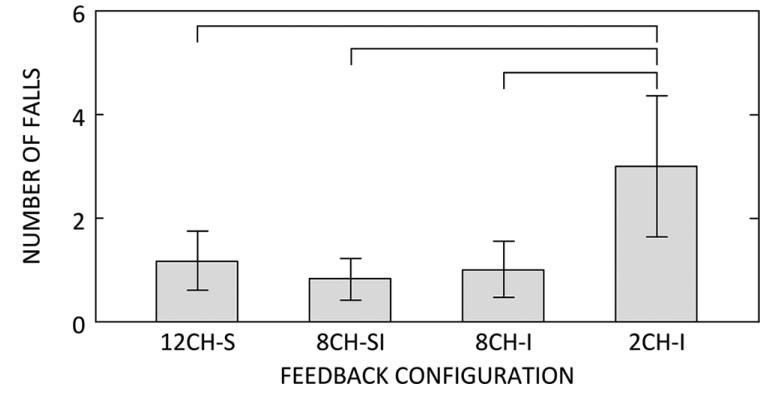

Fig. 5. Results of the pendulum stabilization task across conditions (mean \pm standard error): (a) average deviation from vertical, and (b) average number of falls. Best and the worst performance resulted when $8 \mathrm{CH}-\mathrm{I}$ and $2 \mathrm{CH}-\mathrm{I}$ feedback configurations were used, respectively. Notation: $12 \mathrm{CH}, 8 \mathrm{CH}$ and $2 \mathrm{CH}$ - the number of channels, S and I-spatial and intensity coding. Horizontal bars indicate statistically significant differences. (a) Average deviation across conditions, (b) Average number of falls across conditions.

In the introductory session, all the subjects experienced the control of the pendulum using electrotactile feedback with only two channels (2CH-I) as very challenging, and this was also reflected in the poor performance. They had almost no control of the pendulum movements, and thereby it would fall within a few seconds. However, in the test session, the performance with the same feedback interface (2CH-I) improved substantially and the improvement was immediate. The average time between falls for $2 \mathrm{CH}$-I in the introductory session was $10 \pm 5$ s and it increased threefold ( $32 \pm 29 \mathrm{~s})$ already for the first five trials with the same interface in the testing session.

2) Comparing Feedforward Interfaces: Two force tracking trials, one using the joystick and the other with the myocontrol, recorded in the same subject are depicted in Fig. 6. With both interfaces, to increase the grasping force, the subject increased the control input gradually, whereas the prosthesis responded to this command abruptly, with sudden jumps in the grasping force. Once the desired level of force was attained, there was no need for the further control input, since the prosthesis was non-backdrivable. Therefore, the subject relaxed the muscles or released the joystick back to the center position. To decrease the grasping force, the subject commanded the hand to open, thereby releasing the grip on the object. This release had to be gradual and the control input was proportional to the hand opening velocity. Therefore, the subject cautiously generated low-level intermittent opening commands [i.e., negative "pulses", Fig. 6(a)]. Sometimes the hand would not respond (control pulse too small), and sometimes it would open too fast (control pulse too high). The latter case would result in an abrupt and large decrease in force. For example, in Fig. 6(b), the force 

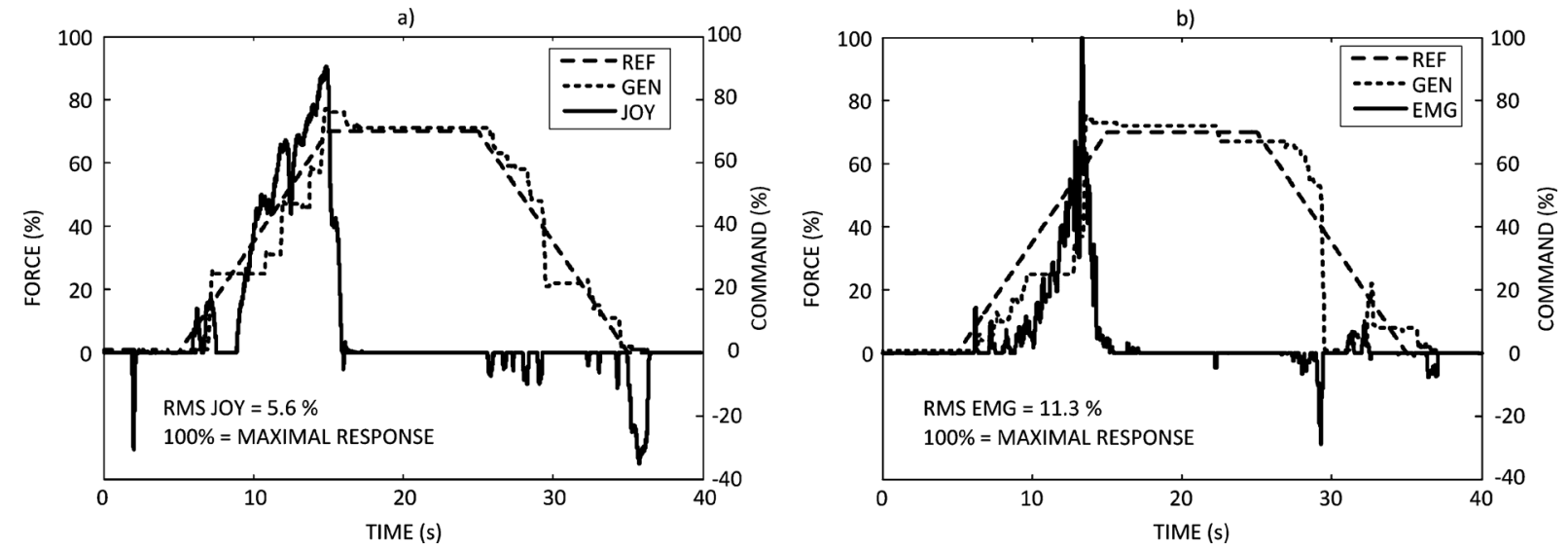

Fig. 6. Representative force tracking trials recorded in the same subject using (a) joystick and (b) myocontrol. Plots depict the reference force profile (REF), generated grasping force (GEN) and the control input (EMG, JOY). Note that the plots have two ordinate axes, left for the force and right for the control. Positive and negative command signal corresponds to the flexor and extensor activity with the myocontrol and left and right inclination of the joystick, respectively. Note that the prosthesis responds with abrupt jumps in the grasping force, leading to a poor tracking performance. (a) Reference and generated force (JOY), (b) Reference and generated force (EMG).

a)

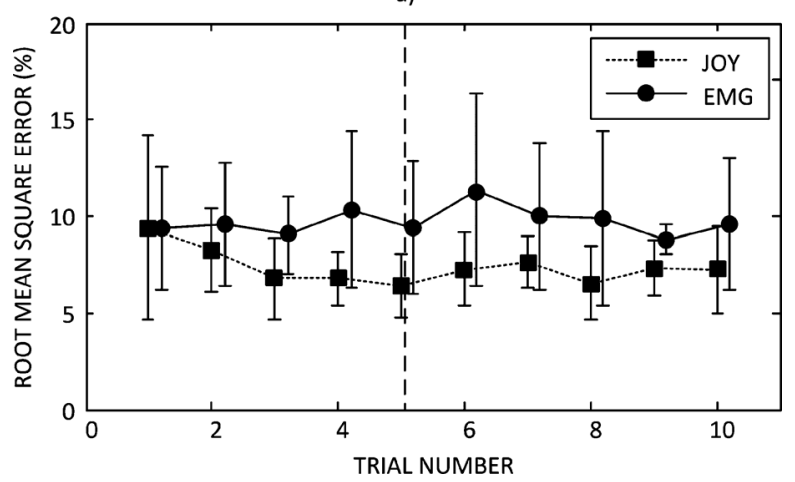

Fig. 7. Average root mean square error (mean \pm standard deviation) for the force tracking task across trials (50\% force target). Performance was overall relatively stable. Vertical dashed line marks the fifth trial. Notation: JOY and EMG denote that the control interface was joystick and myocontrol, respectively. (a) Average root mean square error per trial.

dropped almost instantly from approximately $50 \%$ all the way down to zero, due to a poorly controlled EMG (i.e., see a sudden and strong pulse just preceding the drop).

The performance across trials for the force tracking task using two feedforward control interfaces is shown in Fig. 7. The performance was relatively stable, with a tendency for some improvement in the first few trials when using joystick. The summary results are shown in Fig. 8. As expected, the average tracking errors were lower when using joystick, and the difference was statistically significant for $50 \%$ and $70 \%$ [Fig. 8(a)]. With both EMG and joystick, the absolute tracking errors, expressed as percent of maximal prosthesis force, increased with the higher target force from $6 \% \pm 2 \%$ to $9 \% \pm 3 \%$ for the joystick and from $9 \% \pm 4 \%$ to $12 \% \pm 4 \%$ for myocontrol [Fig. 8(a)]. However, when normalized to the target level, the tracking errors decreased with force from $21 \% \pm 6 \%$ and $29 \% \pm 12 \%$ to $12 \% \pm 4 \%$ and $17 \% \pm 5 \%$ for the joystick and EMG, respectively [Fig. 8(b)]. Overall, the mean tracking errors achieved with the joystick and EMG were not as different, i.e., there was less than $4 \%$ of difference in the absolute errors in all the conditions [Fig. 8(a)].

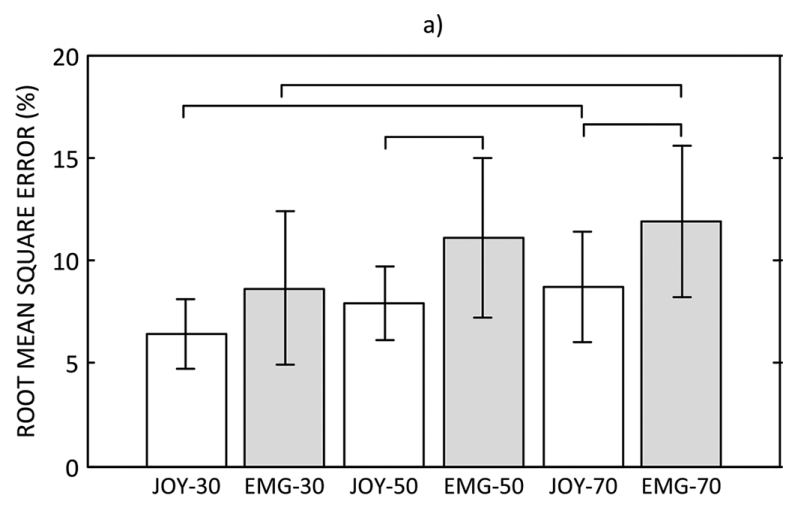

b)

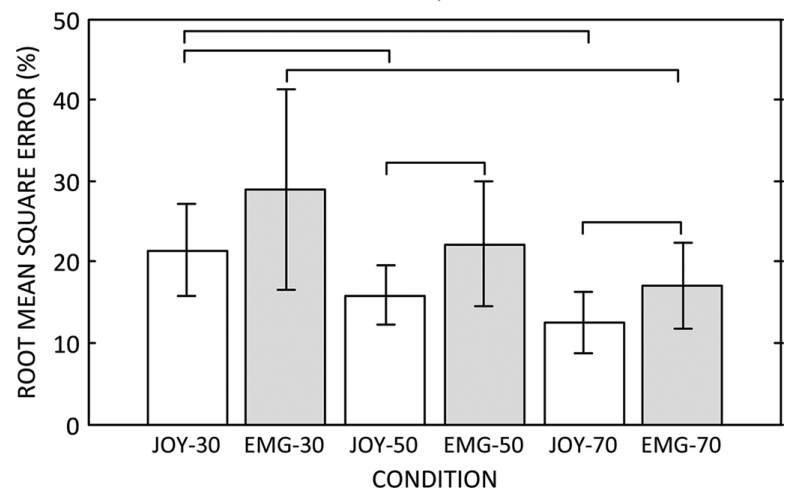

Fig. 8. (a) Absolute and (b) relative average root mean square error (mean \pm standard deviation) for the tracking task across conditions. Joystick control resulted in better tracking compared to EMG, and the absolute tracking error increased for the higher target force levels. Notation: JOY and EMG - control interface, 30, 50, and 70 - the target force level in percent of maximal prosthesis force. Horizontal bars indicate statistically significant differences. To reduce the clutter, only statistical differences between JOY-X versus JOY-Y, EMG-X versus EMG-Y and JOY-X versus EMG-X were depicted ( $\mathrm{X}$ and $\mathrm{Y}$ denote 30, 50, and 70). (a) Absolute error across conditions, (b) Relative error across conditions.

Fig. 9 depicts the absolute tracking errors separately for the force increase [Fig. 9(a)] and decrease [Fig. 9(b)], i.e., positive and negative slope in the reference trajectory (Fig. 6). The error values for both tracking directions exhibit similar trends across increasing target forces. However, for the negative slope, 
a)

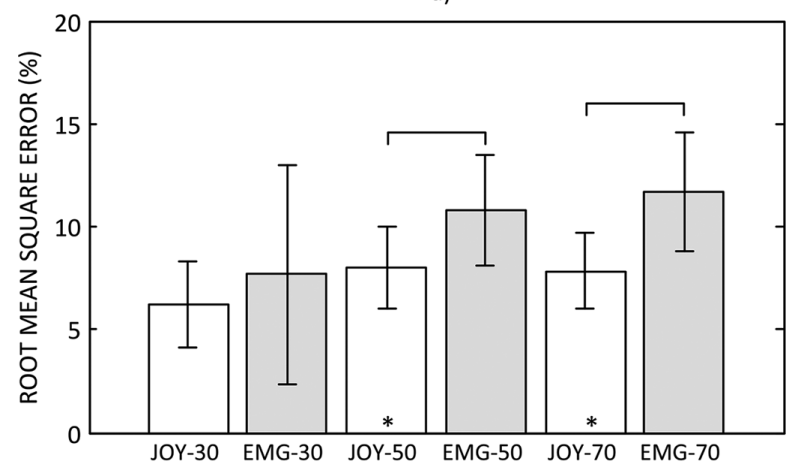

b)

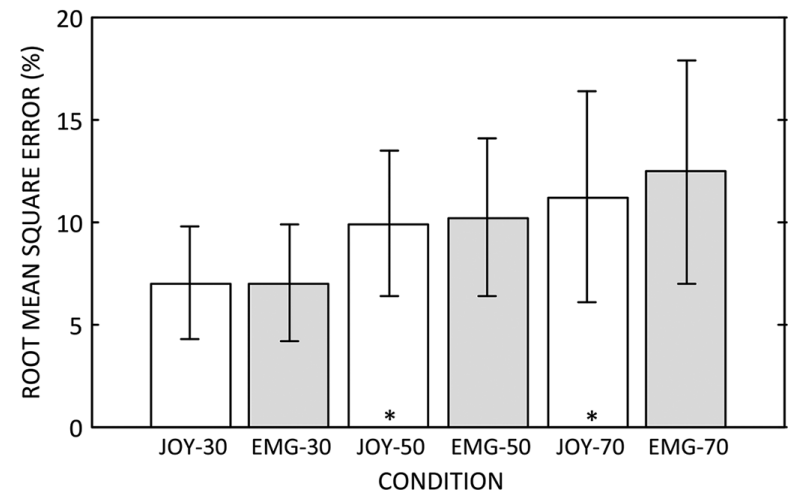

Fig. 9. Absolute average root mean square errors (mean \pm standard deviation) during the tracking of the (a) positive slopes (force increase) and (b) negative slopes (force decrease) of the reference force profile. Note that there were no significant differences between the joystick and EMG during the force decrease. Notation: JOY and EMG - control interface, 30, 50, and 70-the target force level in percent of maximal prosthesis force. Horizontal bars indicate statistically significant differences between the conditions for the same slope (i.e., JOY-X versus EMG-X) and asterisks are for the significant differences between the same conditions for different slopes (i.e., JOY-X for increase versus JOY-X for decrease, and EMG-X for increase versus EMG-X for decrease). (a) Absolute error (force increase), (b) Absolute error (force decrease).

there is no difference in performance between the joystick and EMG (compare Figs. 9(b) to (a) and Fig. 8). The performance with myocontrol was similar for the force increase and decrease, whereas with the joystick the tracking got significantly worse for the negative slope.

\section{DISCUSSION}

1) Closed-Loop Test Bench: We have presented a test bench for the evaluation of closed-loop prosthetic systems in standardized settings. The framework includes a number of ready-to-use components supporting many possible testing scenarios which are configured effortlessly through a few mouse clicks. The test bench was demonstrated by conducting pilot tests evaluating two important aspects of a closed-loop prosthetic system: 1) the configuration of the feedback and 2) the influence of the feedforward interface combined with the inherent characteristics of the prosthesis.

Note that to switch from the control of a pendulum to the control of a real prosthesis, as described in the tests, the user needs to change only one block of the closed-loop framework, i.e., the controlled system. With this simple change, a joystick driven pendulum with electrotactile feedback on inclination turns into a joystick operated prosthesis in which the same electrotactile interface provides information on the prosthesis aperture or force. Due to the virtue of well-defined interfaces between the blocks, the framework integrates different methods and technologies, allowing numerous possibilities to customize the system in a simple way. Myocontrolled prosthesis can be replaced by an EMG-driven pendulum, electrotactile feedback can be substituted by a vibration feedback, and a sequential control using two-channel EMG or a single axis joystick with co-activation (co-contractions) can be replaced by a fully proportional and simultaneous control of two DoFs using a multi axes USB joystick. The framework therefore saves considerable time and effort compared to a conventional approach, which would imply the full development of the components and/or their reintegration into different control configurations. Also, when an additional component is developed (e.g., a novel input interface), the framework provides all the other elements necessary to close the loop. Therefore, the new component can be deployed and tested.

2) Comparing Feedback Interfaces: The comparison of feedback interfaces demonstrated that the intensity coding could be superior to spatial and combined spatial-intensity coding. Spatial coding is intuitive, since the subjects can easily perceive and discriminate the feedback information, but the spatial code is inherently discrete and therefore provides a low resolution. On the other side, the intensity coding provides a virtually continuous representation of the system state. When the subject is trained and therefore familiar enough with the system dynamics, he/she can exploit the higher resolution of the intensity modulation to improve the control. However, the intensity modulated information is harder to perceive correctly, and therefore in order to be effective, it has to be properly amplified. When only one stimulation channel $(2 \mathrm{CH}-\mathrm{I})$ was used instead of four ( $8 \mathrm{CH}-\mathrm{I})$, the performance had changed from overall best to overall worst, although in both cases the same coding was used to deliver the same information. i.e., the only difference being the total area of the skin that was stimulated. Finally, in the case of the combined coding, the spatial information was again easy to interpret, but the intensity was still modulated using only one electrode. It was therefore difficult for the subject to exploit the latter and improve the control (8CH-SI versus $8 \mathrm{CH}-\mathrm{I})$. In the current experiment, we opted for the spatial and intensity coding. Frequency modulation could have been also used to communicate the information with high resolution [19]. Both intensity and frequency coding rely on the physiological mechanisms for information representation, i.e., nerve fiber recruitment and rate of firing, respectively, and both were previously used for trans- [20] as well as sub-cutaneous [21], [22] implementation of artificial sensory feedback. It would be relevant to compare which of these two methods leads to a better performance during closed-loop control tasks, especially since there are psychometric studies implying that human subjects might be more sensitive to changes in the pulse rate [23]. The choice of the modulation method depends on many confounding factors (e.g., stimulator capabilities, subject preferences) and objective pros and cons are yet to be determined.

The current experiments provide optimistic implications for the potentials of electrotactile feedback in system control. 
Namely, they demonstrate that the subjects could successfully accomplish a demanding control task, i.e., managing an unstable system, although they used a nonconventional source of feedback, i.e., a cutaneous sensation communicating the spatial variable (pendulum angle). However, the tests also revealed that the control strategy could change substantially when switching from visual to electrotactile feedback. The latter introduces considerable delays [24], due to uncertainties in cognitive perception and processing of the elicited stimuli. In order to cope with these limitations, the subjects assumed an intermittent style of control. They delivered a short control action (a pulse) and then waited to assess the outcome. This method is known in the system control theory as an approach to deal with feedback delays [25], and it is also hypothesized that intermittent processes operate in the human motor control [26]. While the intermittency seems to be a matter of choice in visual tasks [18], it might be the only feasible approach with electrotactile feedback (at least if not extensively trained).

All these observations have also practical implications. First, they demonstrate a potential training paradigm for electrotactile system control. In the introductory session, the subjects were unable to achieve any meaningful control using the most challenging electrotactile feedback configuration (2CH-I). They also reported general confusion and characterized the task as too difficult. However, after being introduced to the feedback interfaces gradually, starting with the most intuitive one (12CH-S), the subjects could handle the most difficult configuration (2CH-I) substantially better when using it for the second time, i.e., at the end of the test session. Next, designing the closed-loop prosthetic systems can directly benefit from understanding and integrating the motor control strategies of the prosthesis users, especially since they might be very different from the conventional control with visual feedback (as revealed by the current experiment). Finally, the experiments demonstrated that in a trained user, the properly amplified intensity coding allows higher precision. Therefore, a feedback interface for a prosthesis can be envisioned combing the coding methods: the same multichannel electrode array could be used with the spatial coding (intuitive, low effort) and then switched by the user into the more demanding but also potentially more precise intensity coding when necessary (e.g., handling a sensitive object).

3) Comparing Feedforward Interfaces: The second test targeted an issue that is currently largely overlooked in the context of closed-loop prosthesis control. Namely, the overall utility and efficacy of the closed-loop control depends strongly on both constituent components: the feedback and the feedforward. The latter is influenced by the selected control method but also by the inherent characteristics of the system that is to be controlled (mechanical design and local control loops). A prosthesis can exhibit a range of effects, from inertia in responding to nonlinear static and dynamic friction [27]. As demonstrated by the experiment, the prosthesis dynamics can be an important factor, placing the upper limit on the quality of the closed-loop control. The use of a stable and precise control interface (joystick) indeed improved the performance of the force tracking task compared to a more noisy method (myocontrol), but this improvement was more or less marginal. Rough nature of the system operation essentially equalized the two control methods, preventing the user in exploiting the better interface to improve the quality of the control. During force steering, the prosthesis responded proportionally to the control input, as conventionally assumed, but the actual behavior was complex and nonlinear. Overall, the fine control was not feasible. Regardless of the control interface, the subjects could obtain only five to six discrete force levels when increasing the force between 0 and $70 \%$ of the maximum. Similarly rough control characterized the force decrease. In addition, very different mechanisms were operating during the force increase versus decrease and this reflected on the control. The prosthesis was more sensitive during the decrease, and large, abrupt drops in force were almost unavoidable in each trial. As a result, a more consistent interface (joystick) did not translate into a better performance. Importantly, the results of the current experiment were recorded from a specific prosthesis, but the conclusions are general, since many myoelectric prosthesis share similar construction. Obviously, providing a sophisticated high-resolution sensory feedback in this context might be superfluous, or even distracting to the user. It seems that an improvement of the feedforward pathway [27] might be essential in order to fully exploit the potential benefits of the feedback.

4) Future Steps: The main purpose of this communication was twofold: 1) to introduce the test bench as an efficient research and development framework, and 2) to demonstrate its application by conducting two experiments addressing important aspects related to feedback and feedforward pathways of a closed-loop system. Both experiments revealed several basic as well as practical insights relevant for the closed loop prosthesis control and worth exploring further. In parallel, the test bench will be developed by adding more components, such as multiple degree of freedom pattern recognition or simultaneous and proportional control [28]. The goal is to have a flexible platform supporting a broad range of psychophysical and closed-loop control studies (e.g., intensity versus frequency modulation, as mentioned before). Currently, the test bench can be obtained by contacting the authors, and will soon be freely available online.

\section{REFERENCES}

[1] M. A. Oskoei and H. Hu, "Myoelectric control systems-A survey," Biomed. Signal Process. Contr., vol. 2, no. 4, pp. 275-294, Oct. 2007.

[2] E. N. Kamavuako, J. C. Rosenvang, R. Horup, W. Jensen, D. Farina, and K. B. Englehart, "Surface versus untargeted intramuscular EMG based classification of simultaneous and dynamically changing movements," IEEE Trans. Neural Syst. Rehabil. Eng., vol. 21, no. 3, p. 1, Mar. 2013.

[3] J. M. Hahne, B. Graimann, and K.-R. Müller, "Spatial filtering for robust myoelectric control," IEEE Trans. Biomed. Eng., vol. 59, no. 5, pp. 1436-1443, May 2012.

[4] Sensor Hand Speed and Michelangelo Hand, [Online]. Available: http:/ /www.ottobock.com/cps/rde/xchg/ob com en/hs.xsl/3633.html.

[5] Bebionic Hand 2013, [Online]. Available: [Online]. Available: http:// bebionic.com/

[6] Touch Bionics iLimb 2013, [Online]. Available: [Online]. Available: $\mathrm{http}: / /$ www.touchbionics.com/

[7] K. A. Kaczmarek, J. G. Webster, P. Bach-y-Rita, and W. J. Tompkins, "Electrotactile and vibrotactile displays for sensory substitution systems," IEEE Trans. Biomed. Eng., vol. 38, no. 1, pp. 1-16, Jan. 1991.

[8] A. Y. Szeto and F. A. Saunders, "Electrocutaneous stimulation for sensory communication in rehabilitation engineering," IEEE Trans. Biomed. Eng., vol. 29, no. 4, pp. 300-308, Apr. 1982.

[9] P. P. Kadkade, B. J. Benda, P. B. Schmidt, and C. Wall, "Vibrotactile display coding for a balance prosthesis," IEEE Trans. Neural Syst. Rehabil. Eng., vol. 11, no. 4, pp. 392-399, Dec. 2003. 
[10] N. D. Jorgovanovic, S. Dosen, D. Djozic, K. Goran, and Farina, "Virtual grasping: Closed-loop force control using electrotactile feedback," Comput. Math. Methods Med., 2013.

[11] C. Antfolk, M. D'Alonzo, B. Rosén, G. Lundborg, F. Sebelius, and C. Cipriani, "Sensory feedback in upper limb prosthetics," Expert Rev. Med. Devices, vol. 10, no. 1, pp. 45-54, Jan. 2013.

[12] C. Cipriani, F. Zaccone, S. Micera, and M. C. Carrozza, "On the shared control of an EMG-controlled prosthetic hand: Analysis of user-prosthesis interaction," IEEE Trans. Robot., vol. 24, no. 1, pp. 170-184, Feb. 2008.

[13] S. G. Meek, S. C. Jacobsen, and P. P. Goulding, "Extended physiologic taction: Design and evaluation of a proportional force feedback system," J. Rehabil. Res. Dev., vol. 26, no. 3, pp. 53-62, Jan. 1989.

[14] A. Chatterjee, P. Chaubey, J. Martin, and N. Thakor, "Testing a prosthetic haptic feedback simulator with an interactive force matching task," JPO J. Prosthetics Orthot., vol. 20, no. 2, pp. 27-34, Apr. 2008.

[15] H. J. B. Witteveen, E. A. Droog, J. S. Rietman, and P. H. Veltink, "Vibro- and electrotactile user feedback on hand opening for myoelectric forearm prostheses," IEEE Trans. Biomed. Eng., vol. 59, no. 8, pp. 2219-2226, Aug. 2012.

[16] A. Ninu, S. Dosen, D. Farina, F. Rattay, and H. Dietl, "A novel wearable vibro-tactile haptic device," 2013.

[17] F. A. A. Kingdom and N. Prins, Psychophysics: A Practical Introduction. New York, NY, USA: Academic, 2009, p. 304.

[18] I. D. Loram, H. Gollee, M. Lakie, and P. J. Gawthrop, "Human control of an inverted pendulum: Is continuous control necessary? Is intermittent control effective? Is intermittent control physiological?," $J$. Physiol., vol. 589, no. Pt 2, pp. 307-324, Jan. 2011.

[19] A. Y. J. Szeto and J. Lyman, "Comparison of codes for sensory feedback using electrocutaneous tracking," Ann. Biomed. Eng., vol. 5, no. 4, pp. 367-383, Dec. 1977

[20] G. F. Shannon, "A comparison of alternative means of providing sensory feedback on upper limb prostheses," Med. Biol. Eng., vol. 14, no. 3, pp. 289-294, May 1976.

[21] S. Raspopovic, M. Capogrosso, F. M. Petrini, M. Bonizzato, J. Rigosa, G. Di Pino, J. Carpaneto, M. Controzzi, T. Boretius, E. Fernandez, G. Granata, C. M. Oddo, L. Citi, A. L. Ciancio, C. Cipriani, M. C. Carrozza, W. Jensen, E. Guglielmelli, T. Stieglitz, P. M. Rossini, and S. Micera, "Restoring natural sensory feedback in real-time bidirectional hand prostheses," Sci. Transl. Med., vol. 6, no. 222, p. 222ra19, Feb. 2014.

[22] G. S. Dhillon and K. W. Horch, "Direct neural sensory feedback and control of a prosthetic arm," IEEE Trans. Neural Syst. Rehabil. Eng., vol. 13 , no. 4, pp. $468-472$, Dec. 2005.

[23] A. Y. J. Szeto, J. Lyman, and R. E. Prior, "Electrocutaneous pulse rate and pulse width psychometric functions for sensory communications," Hum. Factors J. Hum. Factors Ergon. Soc., vol. 21, no. 2, pp. 241-249, Apr. 1979.

[24] H. P. Schmid and G. A. Bekey, "Tactile information processing by human operators in control systems," IEEE Trans. Syst. Man. Cybern., vol. 8 , no. 12 , pp. 860-866, Dec. 1978.

[25] T. Insperger, "Act-and-wait concept for continuous-time control systems with feedback delay," IEEE Trans. Contr. Syst. Technol., vol. 14, no. 5, pp. 974-977, Sep. 2006.

[26] P. Gawthrop, I. Loram, M. Lakie, and H. Gollee, "Intermittent control: A computational theory of human control," Biol. Cybern., vol. 104, no. $1-2$, pp. 31-51, Feb. 2011.

[27] E. D. Engeberg and S. Meek, "Improved grasp force sensitivity for prosthetic hands through force-derivative feedback," IEEE Trans. Biomed. Eng., vol. 55, no. 2 Pt 2, pp. 817-821, Mar. 2008.

[28] N. Jiang, K. B. Englehart, and P. A. Parker, "Extracting simultaneous and proportional neural control information for multiple-DOF prostheses from the surface electromyographic signal," IEEE Trans. Biomed. Eng., vol. 56, no. 4, pp. 1070-1080, Apr. 2009.

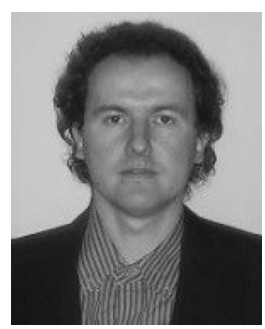

Strahinja Dosen (M'05) received the Diploma of Engineering in electrical engineering and the M.Sc. degree in biomedical engineering in 2000 and 2004 , respectively, from the Faculty of Technical Sciences, University of Novi Sad, Serbia, and the Ph.D. degree in biomedical engineering from Aalborg University, Aalborg, Denmark, in 2008.

Currently, he is a Research Scientist at the Department of Neurorehabilitation Engineering, University Medical Center Göttingen (UMG), Göttingen, Germany. His main research interest is the closed-loop control of movements and assistive systems.

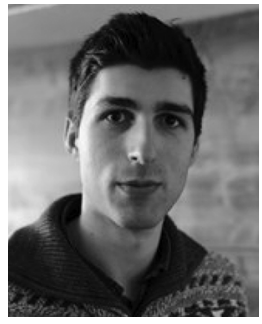

Marko Markovic received the Master of Engineering degree at the University of Belgrade, Serbia, in 2011. He is working towards the Ph.D. degree at University Medical Center Göttingen (UMG), Göttingen, Germany.

Between 2012 and 2014, he was employed as a Research Assistant at the Department of Neurorehabilitation Engineering, University Medical Center Goettingen, Germany. Currently, he is employed by Ottobock HealthCare $\mathrm{GmbH}$ as a MYOSENS-project Fellow. His research is focused on the evaluation and usage of the upper limb prosthetic devices in closed-loop control scenarios. Specifically, these topics include: development of flexible training paradigms together with novel feedback modalities, and exploiting modern computer vision algorithms for dexterous prosthesis control.

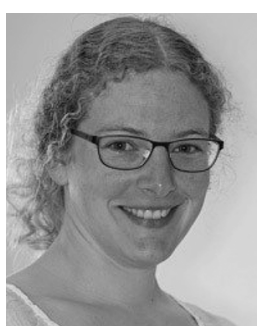

Cornelia Hartmann (S'14) received the B.Sc degree and Diploma in electrical engineering and information technology from Technische Universität München, Munich, Germany, in 2006 and 2008, respectively. She is working toward the Ph.D. degree in theoretical and computational neuroscience at the Department of Neurorehabilitation Engineering, University Medical Center Göttingen, Georg August University, Göttingen, Germany.

From 2008 to 2012, she was with the Research and Development Department of Otto Bock Healthcare $\mathrm{GmbH}$, Germany. Her research interests include closed-loop prosthesis control as well as sensory feedback for prosthesis users.

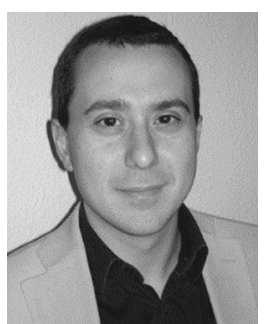

Dario Farina (M'01-SM'09) received the M.Sc. degree in electronics engineering from Politecnico di Torino, Torino, Italy, in 1998, and the Ph.D. degree in automatic control and computer science and in electronics and communications engineering from the Ecole Centrale de Nantes, Nantes, France, and Politecnico di Torino, respectively, in 2002.

During 2002 to 2004, he was a Research Assistant Professor at Politecnico di Torino and in 2004 through 2008 an Associate Professor in Biomedical Engineering at Aalborg University, Aalborg, Denmark. From 2008 to 2010, he was a Full Professor in Motor Control and Biomedical Signal Processing and Head of the Research Group on Neural Engineering and Neurophysiology of Movement at Aalborg University. In 2010, he was appointed Full Professor and Founding Chair of the Department of Neurorehabilitation Engineering at the University Medical Center Göttingen, Georg-August University, Germany, within the Bernstein Center for Computational Neuroscience. He is also the Chair for NeuroInformatics of the Bernstein Focus Neurotechnology Göttingen. His research focuses on biomedical signal processing, modeling, neurorehabilitation technology, and neural control of movement. Within these areas, he has (co)-authored approximately 350 papers in peer-reviewed journals and over 400 among conference papers/abstracts, book chapters and encyclopedia contributions.

Dr. Farina has been the President of the International Society of Electrophysiology and Kinesiology (ISEK) from 2012 and 2014. He is the recipient of the 2010 IEEE Engineering in Medicine and Biology Society Early Career Achievement Award for his contributions to biomedical signal processing and to electrophysiology and in 2012 he was elected Fellow of the American Institute for Medical and Biological Engineering (AIMBE) for his contributions to neurotechnologies. He is an Associate Editor of IEEE TRANSACTIONS ON BIOMEDICAL ENGINEERING. 


\section{APPENDIX 4: EMG BIOFEEDBACK FOR ONLINE PREDICTIVE CONTROL OF GRASPING FORCE IN A MYOELECTRIC PROSTHESIS}


Sensor Fusion for Closed-loop Control of Upper-limb Prostheses 


\title{
EMG Biofeedback for online predictive control of grasping force in a myoelectric prosthesis
}

\author{
Strahinja Dosen ${ }^{1}$, Marko Markovic $^{2}$, Kelef Somer', Bernhard Graimann² and Dario Farina ${ }^{1 *}$
}

\begin{abstract}
Background: Active hand prostheses controlled using electromyography (EMG) signals have been used for decades to restore the grasping function, lost after an amputation. Although myocontrol is a simple and intuitive interface, it is also imprecise due to the stochastic nature of the EMG recorded using surface electrodes. Furthermore, the sensory feedback from the prosthesis to the user is still missing. In this study, we present a novel concept to close the loop in myoelectric prostheses. In addition to conveying the grasping force (system output), we provided to the user the online information about the system input (EMG biofeedback).

Methods: As a proof-of-concept, the EMG biofeedback was transmitted in the current study using a visual interface (ideal condition). Ten able-bodied subjects and two amputees controlled a state-of-the-art myoelectric prosthesis in routine grasping and force steering tasks using EMG and force feedback (novel approach) and force feedback only (classic approach). The outcome measures were the variability of the generated forces and absolute deviation from the target levels in the routine grasping task, and the root mean square tracking error and the number of sudden drops in the force steering task.

Results: During the routine grasping, the novel method when used by able-bodied subjects decreased twofold the force dispersion as well as absolute deviations from the target force levels, and also resulted in a more accurate and stable tracking of the reference force profiles during the force steering. Furthermore, the force variability during routine grasping did not increase for the higher target forces with EMG biofeedback. The trend was similar in the two amputees.

Conclusions: The study demonstrated that the subjects, including the two experienced users of a myoelectric prosthesis, were able to exploit the online EMG biofeedback to observe and modulate the myoelectric signals, generating thereby more consistent commands. This allowed them to control the force predictively (routine grasping) and with a finer resolution (force steering). The future step will be to implement this promising and simple approach using an electrotactile interface. A prosthesis with a reliable response, following faithfully user intentions, would improve the utility during daily-life use and also facilitate the embodiment of the assistive system.
\end{abstract}

Keywords: Closed-loop prosthesis control, Myoelectric prosthesis, EMG biofeedback, Sensory feedback, Routine grasping, Force steering, Grasping consistency

\section{Background}

Human hand is a dexterous end-effector and a sophisticated instrument for sensory exploration [1]. After an amputation, these important motor and sensory functions are abruptly lost. Myoelectric hand prostheses can be used to restore grasping. The control signal (input voltage) driving the prosthesis motor is obtained by applying simple processing (smoothing) to the electromyography (EMG) signals

\footnotetext{
* Correspondence: dario.farina@bccn.uni-goettingen.de

${ }^{1}$ Department of Neurorehabilitation Engineering, University Medical Center Göttingen (UMG), Georg-August University, 37075 Göttingen, Germany Full list of author information is available at the end of the article
}

recorded from the user muscles. The commercial state-ofthe-art myoelectric interface uses two channels of EMG: the activity of hand and wrist flexor muscles is proportional to the prosthesis closing speed and grasping force, while the extensor activity controls proportionally the speed of opening $[2,3]$. Therefore, the commercial myoelectric prostheses provide the grasping function by restoring the feedforward pathway between the user's brain and the artificial hand, but there is no sensory feedback from the prosthesis to the user. There is only one commercially available system [4], presented recently, implementing a simple feedback about the
Ciomed Central (c) 2015 Dosen et al. This is an Open Access article distributed under the terms of the Creative Commons Attribution License (http://creativecommons.org/licenses/by/4.0), which permits unrestricted use, distribution, and reproduction in any medium, provided the original work is properly credited. The Creative Commons Public Domain Dedication waiver (http:// creativecommons.org/publicdomain/zero/1.0/) applies to the data made available in this article, unless otherwise stated. 
hand grasping force. In principle, sensor data can be transmitted from the prosthesis to the user invasively, through a direct stimulation of the nerves [5], and non-invasively, by electrically [6] and/or mechanically [7] stimulating the skin. Closing the loop in myoelectric prostheses was acknowledged as an important future goal by the prospective users as well as researchers in the field [8]. Sensory feedback might improve the utility of the assistive devices as well as facilitate the embodiment [9].

Two-channel myoelectric interface is a simple and intuitive control method since the user operates the prosthesis by activating the same muscles (finger flexors/extensors) that were responsible for those functions (hand open/close) before the amputation. However, the EMG signals acquired using surface electrodes are noisy and variable, due to inherent limitations of the recording setup (e.g., detection separated from the signal source), and the control is thereby rather imprecise [10]. For this reason, as demonstrated in [11], the prosthesis may respond inconsistently to the user intentions. Repeatedly closing the prosthesis to generate the same grasping force was characterized with a large variability, which also increased with higher target forces. The subjects could not repeat muscle contractions in a reliable manner using the natural proprioceptive feedback from own muscles to provide consistent control signals. Imprecise control can produce user frustration, often leading to the abandonment of the prosthesis [12]. Furthermore, it can be a limiting factor for the effectiveness of the sensory feedback [11]. Indeed, it can be rather useless for the user to sense the state of the system (e.g., aperture or grasping force), if he/she cannot produce a sequence of commands driving the prosthesis reliably towards the desired state (e.g., target aperture or grasping force). Improving the consistency of the command is thereby an extremely relevant goal. A reliable control loop would allow the benefits of the sensory feedback to be fully expressed. A well-controllable prosthesis following faithfully the user intentions would also better emulate the operation of its biological counterpart, potentially facilitating embodiment.

In the current study, we propose a novel concept for closing the loop in myoelectric prostheses, designed specifically to improve the consistency of the prosthesis response by allowing the user to reduce the variability of the control signals he/she generates by muscle activation. The new approach was tested experimentally and the tests demonstrated that it significantly improved the performance both in routine grasping and force steering tasks.

\section{Methods \\ Proposed method \\ General concept}

In the classic approach to closing the loop in myoelectric prostheses, the system output (e.g., grasping force) is delivered to the user. The novel concept proposed and investigated in this study (Fig. 1) is to provide feedback on the control input that the user generates (prosthesis command) in addition to the consequence of such input (grasping force). Specifically, the generated and processed myoelectric signals are transmitted to the prosthesis as commands and simultaneously to the user as online feedback information. In the conventional approach to prosthesis control, the myoelectric signals are latent variables, whereas in the novel scheme (Fig. 1) these signals become explicit (observable) through the application of the EMG biofeedback. The user can therefore modulate the control input by using a local closed

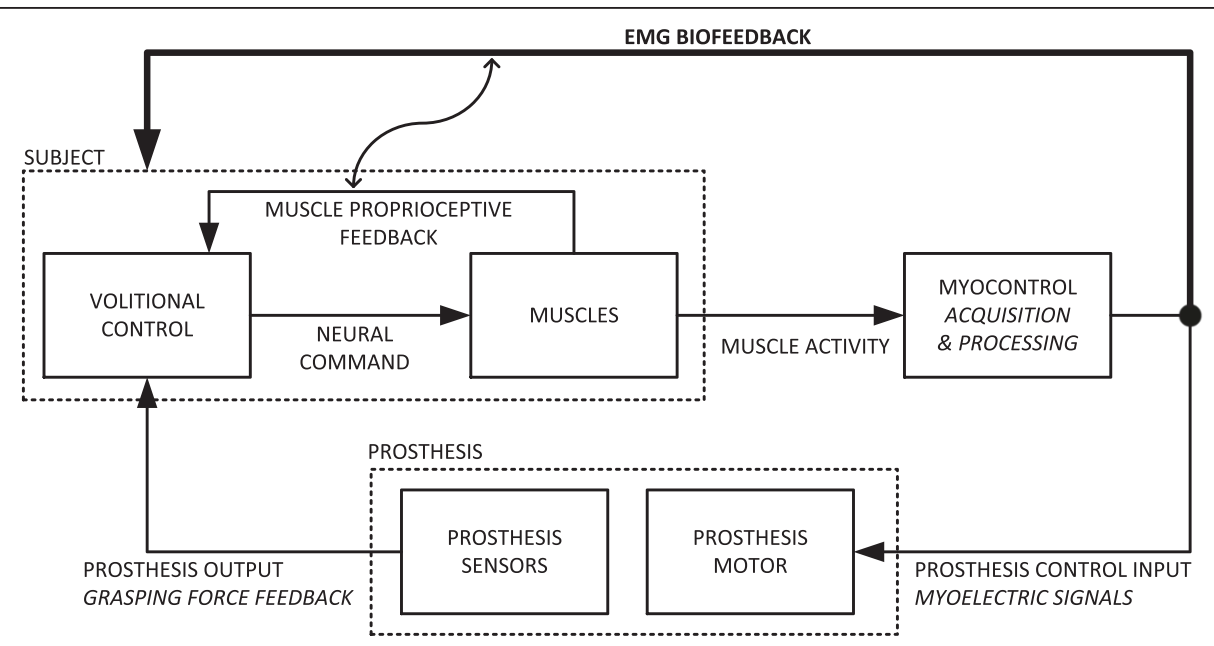

Fig. 1 Conceptual scheme for the application of EMG biofeedback to improve force control in myoelectric prostheses. The user receives online information about the level of the myoelectric signals he/she generates. Since the grasping force is approximately proportional to the input myoelectric signals at the moment of contact, the user can control the grasping force predictively, i.e., by adjusting his/her myoelectric signals during the prosthesis closing. EMG biofeedback can also facilitate the modulation of the grasping force once the hand is closed (see text) 
loop (bold line in Fig. 1), allowing him/her to produce consistent and reproducible commands, actively compensating for the inherent variability of the surface myoelectric interface. The proposed method was tested in two representative prosthesis control tasks, namely, routine grasping and force steering.

\section{Routine grasping task}

Routine grasping refers to a smooth and straightforward closing of the prosthesis so that the desired grasping force is reached immediately after contacting the object, avoiding thereby a careful (and tedious) adjustment of the prosthesis force [11]. This resembles the way in which able-bodied persons grasp objects in daily life. When using a prosthesis, the routine grasping is accomplished by generating and holding a certain level of muscle contraction (as a percent of the maximum voluntary contraction, MVC); since the closing speed and grasping force are proportional to the command input, the prosthesis closes at a certain speed (as a percent of the maximum speed), which becomes "converted" into a corresponding force (percent of the maximum force) once the motor stalls (contact with the object). In a conventional closed-loop system, the user regulates his/her myoelectric output indirectly, by modulating the intensity of contraction relying solely on the proprioceptive feedback from own muscles. The user is therefore unaware of the exact control signal that is being delivered to the prosthesis. Only after contact, the user receives the force feedback, which also reveals the actual command that was applied to the prosthesis during closing; however, this information comes too late since the grasp is already formed (e.g., object broken due to an excessive force). When the EMG biofeedback is provided, as proposed in the novel scheme (Fig. 1), the task becomes explicit. The user is able to modulate the muscle activity reaching the desired signal level (as a percent of MVC) and then maintain that level by relying on the EMG biofeedback closed loop. The hand starts closing, and the user, by monitoring and controlling his/her myoelectric activity, predictively controls the level of force that will be generated once the object is contacted (grasped).

\section{Force steering task}

In this task, the aim is to modulate the grasping force while the hand is closed around an object (e.g., grasping an object and then strengthening the grip) [13]. There are two mechanisms characteristic for myoelectric prostheses making the modulation of force challenging.

First, the prosthesis is non-backdrivable, allowing the user to relax the muscles while the prosthesis continues holding the attained level of force. This frees the user from having to maintain a prolonged muscle contraction. When the force needs to be increased, however, the user must activate the flexor muscle and increase the contraction from the resting state until the control signal is higher than the level corresponding to the current grasping force. Since in the classic control scheme, the user does not know the exact value of the control signal that is being generated, he/she cannot be sure when the prosthesis will start reacting. Therefore, the eventual increase in force often comes as a surprise, leading to a poor control of the force increments. By providing the EMG biofeedback, the moment the prosthesis will respond becomes explicit, since the user can monitor online (and precisely modulate) how the control input approaches the current level of force.

Second, a completely different mechanism is active when decreasing the force. In this case, the user releases the grip by commanding the prosthesis to open, where the velocity of opening is proportional to the myoelectric signal recorded from the extensor muscle. In order to decrease the force gradually, the hand must be opened very slowly, by activating the extensor just above the threshold level. Again, in the classic approach, this is difficult to accomplish since the current level of the generated myoelectric signal is unknown to the user. Often, the prosthesis force suddenly drops to zero as a result of a higher extensor activation, which opens the hand and breaks the contact with the object. Again, with the EMG biofeedback, the user can fine-tune the low-level control signal and thereby decrease the force gradually and in a controllable manner.

In both of these tasks (force steering and routine grasping), the EMG biofeedback can be regarded as assisting and enhancing already existing natural proprioceptive feedback from the muscles, which alone is not a reliable indication of the level of muscle contraction and prosthesis response [11]. In that sense, the EMG biofeedback can be applied as a training instrument facilitating the subject to better utilize (interpret) the natural muscle proprioceptive feedback for prosthesis control. This is denoted in Fig. 1 by the "S"-shaped arrow connecting the two feedback channels. However, this potential application of the EMG biofeedback was outside the scope of the current study.

\section{Experimental setup and protocol}

The setup comprised: 1) Michelangelo Hand prosthesis (Otto Bock Healthcare Products GmbH, Vienna, AT), 2) EMG amplifier (INTEMG, OTBioelettronica, IT), and 3) a standard desktop computer with a 22" screen. Figure 2 depicts the components and the control loop as it was implemented in the real-time framework for the assessment of the manual closed-loop control systems [14].

The Michelangelo Hand [15] is a two degree-of-freedom prosthesis with mechanically-coupled fingers flexing and extending around the metacarpophalangeal joints plus the thumb which can also move into opposition. Therefore, the 


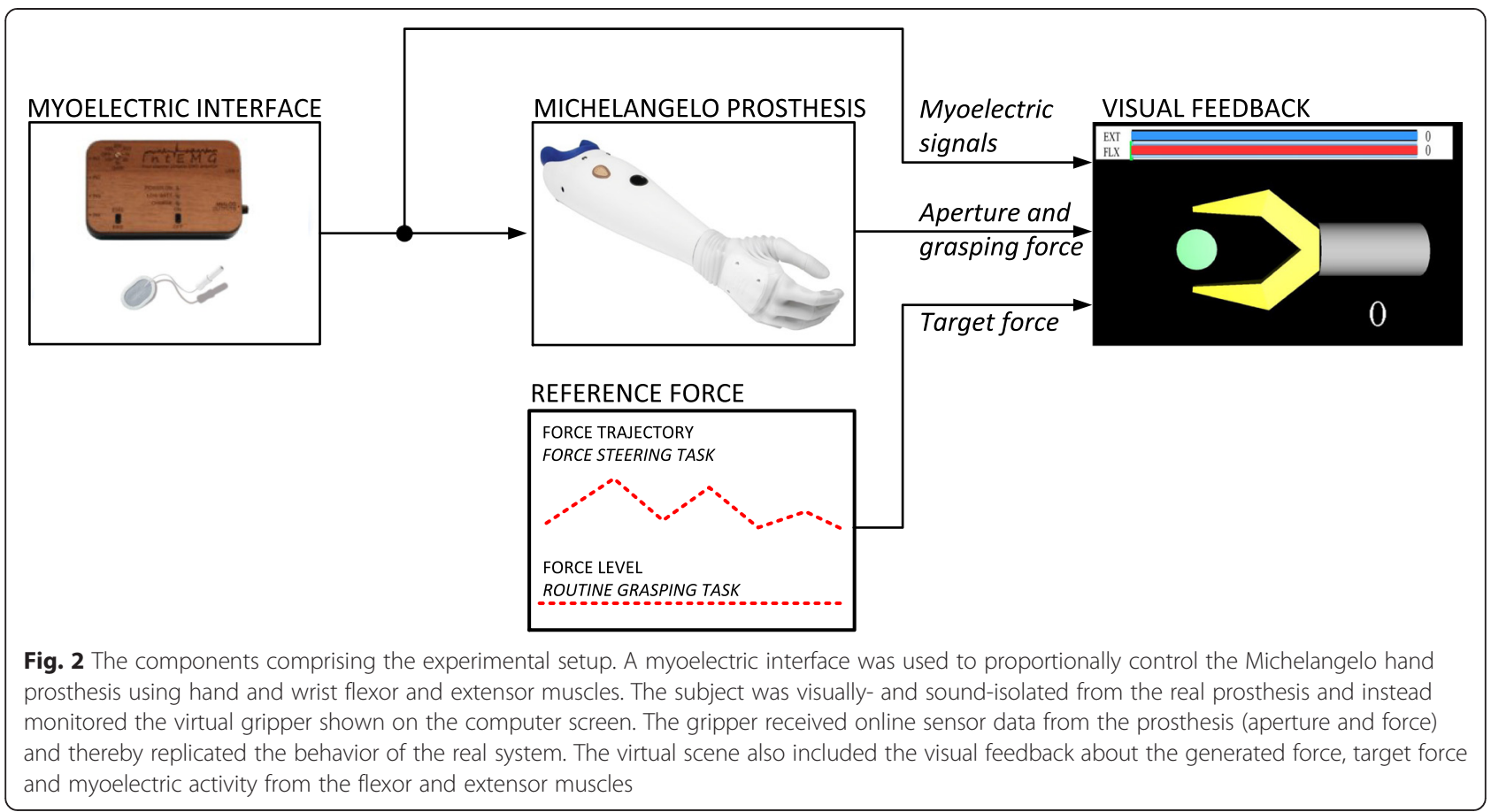

hand can implement lateral (between the thumb and index) and pinch (between fingertips) grasps, where only the latter was used in the current study. The hand integrates a Bluetooth interface through which a normalized command signal can be sent to the prosthesis. The hand response profiles mapping the constant command input to the closing speed and grasping force, respectively, were recorded and then linearized to obtain an ideal correspondence (i.e., $\mathrm{X} \%$ of $\mathrm{MVC} \Rightarrow \mathrm{X} \%$ of maximum speed $\Rightarrow \mathrm{X} \%$ of maximum force). Two channels of bipolar EMG were recorded from the hand and wrist flexor and extensor muscles, proportionally controlling the hand closing/opening and grasping force. Standard pre-gelled $\mathrm{Ag} / \mathrm{AgCl}$ electrodes were used (Neuroline 720, Ambu, US). A stiff cylindrical object was positioned and secured between the prosthesis fingers so that the hand grasped it when closed. During the experiment, the prosthesis and the object were placed in another room, while the subjects were looking into the computer screen showing a geometrical model of a simple gripper grasping a stiff cylinder (Fig. 3). Therefore, the subjects controlled the real prosthesis (Michelangelo Hand) through the myoelectric interface. The prosthesis sensor data (position and force) were sampled internally by the embedded controller $(100 \mathrm{~Hz})$ and then sent to the host PC to update the visual feedback displayed on the computer screen. The gripper replicated the movement (aperture) of the prosthesis and the grasping force was displayed using a horizontal bar, as described below. The setup provided a standardized feedback across subjects and conditions. By detaching the subjects from the prosthesis, some sources of feedback were eliminated (e.g., motor/mechanism sound, haptic feedback through the socket, deformation of the silicone skin when grasping an object). However, as in a reallife application, the subjects could still monitor the prosthesis movements, and the setup was configured specifically to facilitate this observation (e.g., clear, lateral view of the prosthesis). This was done considering that the prosthesis closing velocity is an important information, since it can be used to control the grasping force predictively, as demonstrated in [11].

The EMG was sampled at $1 \mathrm{kHz}$ and its root mean square was computed over time intervals of $250 \mathrm{~ms}$ and with $90 \%$ of overlap. The control loop running at the PC (Fig. 1) operated at $200 \mathrm{~Hz}$. The acquired data from the EMG amplifier were sent to the PC via USB, filtered using a first-order low-pass Butterworth filter with a cutoff at $1 \mathrm{~Hz}$, and finally thresholded and normalized to the interval $[0,1]$, where 0 and 1 corresponded to the sub-threshold activity and $70 \%$ of $\mathrm{MVC}$, respectively. This was done in order to map the prosthesis force range to the user sense of effort (high force, high effort) but still avoid fatigue during repeated contractions. The exact level used in the present experiment was adopted based on pilot tests, since to our knowledge there are no studies investigating the optimal mapping between the prosthesis force and user myoelectric range. In practice, amputees adjust this mapping according to personal preferences by, for example, turning a potentiometer on the electrode (Otto Bock systems). The resulting command signal was sent to the hand prosthesis and to the block implementing the virtual scene on the computer screen. The scene (Fig. 3) included a geometrical model 


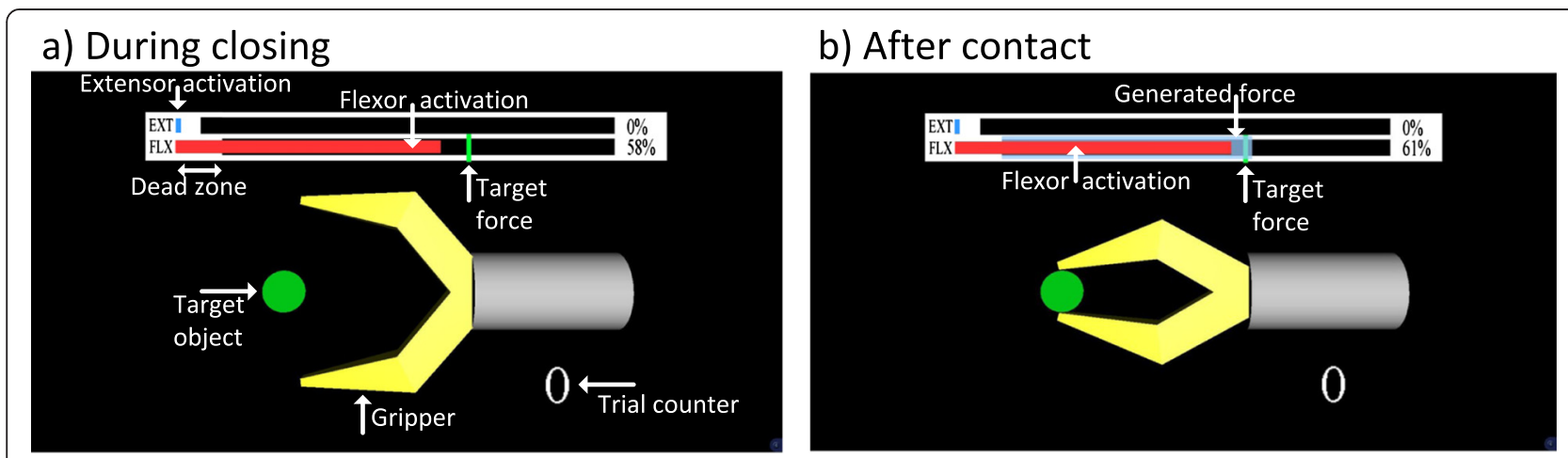

Fig. 3 Visual scene shown to the subjects during the experiments including a snapshot of the screen (a) before contact and (b) after contact. The real prosthesis (Michelangelo hand) hidden from the subjects' view grasped a stiff cylindrical object, and this was presented to the subject in the form of a virtual gripper grasping a virtual target object. Horizontal bars, red for the flexor and blue for the extensor, showed a continuous feedback about the current level of muscle activity (prosthesis control signals). As long as the myoelectric bars did not reach the respective black lines, the myoelectric activity was subthreshold (i.e., a dead zone area resulting in zero control input to the prosthesis). Semi-transparent blue bar indicated the hand grasping force and the green vertical line was the target force level. During the routine grasping, the target force was stationary, while in the force steering task, it was moving according to the time profile of a reference force trajectory

of a simple gripper and horizontal bars just above the gripper, providing the current value of the flexor and extensor myoelectric signals (EMG biofeedback) as well as the generated and target grasping force (classic force feedback). Note that during the prosthesis closing (Fig. 3[a]), while the grasping force was zero, the subject activated his/her flexor muscle so that the generated myoelectric signal (red bar) was close to the target force level (green line). As a result, after contact (Fig. 3[b]), the generated grasping force (semi-transparent blue bar) reached close to the desired level. Once the grasp was formed, the prosthesis reacted by increasing the force only when the myoelectric activity was higher than the current force level (i.e., red bar overtaking the semitransparent blue bar); otherwise, the prosthesis held the current force due to the non-backdrivable operation. Similarly, the prosthesis started decreasing force only after the extensor signal crossed the dead-zone threshold (i.e., blue bar crossing into the respective black line). As explained before, the aim of the current study was to present and for the first time test the validity and benefit of the novel approach. Therefore, an ideal visual feedback was used to transmit the information to the user. However, the information transmission can be easily translated into another modality, e.g., electro- or vibrotactile, as discussed later.

Ten able-bodied subjects ( $23 \pm 3$ years) and two amputees (55 and 43 years) participated in the study, and signed the informed consent forms for the experiment approved by the Ethical Committee of the University Medical Center Göttingen. First amputee, hereafter denoted as amputee 1, was an experienced and active user (50 h/week) of a myoelectric prosthesis (Sensor Hand, Otto Bock), with the left hand amputated 30 years ago at the transradial level. Second amputee (amputee 2) was congenital (wrist level, right hand), and also experienced but occasional user (10 h/week) of the same type of myoelectric prosthesis. The subjects were comfortably seated in a chair in front of a table, looking into a computer screen positioned approximately $50 \mathrm{~cm}$ away. The positions for the placement of the EMG electrodes were determined by palpating and visually observing muscle contractions in the dominant forearm of able-bodied subjects and residual limb of amputees, and the skin was prepared with a small amount of abrasive gel (everi, Spes Medica, IT). The forearm and hand of able-bodied subjects were placed within an orthopedic splint so that the subjects controlled the prosthesis by generating nearly isometric muscle contractions. The arm was held in a self-selected comfortable position (e.g., vertically next to the trunk or on the table). The principle of prosthesis operation was explained to the subjects and they were allowed to practice both tasks for a short time (10-15 min).

The task for the subjects during the routine grasping test was to close the prosthetic hand from the fully open position, grasp the object and reach the desired level of force as indicated by the target force bar. The subject then relaxed the muscles to mark the end of the trial, and this triggered an automatic opening of the hand. The maximum force attained during the trial was adopted as the trial outcome. The subjects were instructed to activate the muscles and close the hand so that the target grasping force was reached directly after contact (no force steering). During training, if the experimenter noticed that the subjects corrected the force after contact, he discouraged them from doing so in the next trials. In addition, the control algorithm ignored any extensor input from the user (no force decrease). The subjects grasped repeatedly in two blocks of 50 trials with the target forces equal to 30, 50 and $70 \%$ of the maximum, with simultaneous EMG 
and force feedback (EMG/FORCE, novel approach) and with force feedback only (FORCE, classic closed-loop scheme). In the latter condition, the bars indicating the current level of muscle activity (Fig. 3, red for flexor, blue for extensor) were not shown. At the beginning of the trial, the target force (vertical green line) was displayed, and after contact, the momentary grasping force (semitransparent blue bar) was indicated to the subject. In total, there were 300 trials in both feedback conditions. The first ten trials in each block were regarded as a warming up and were not used for data analysis. Due to a routine grasping paradigm, the trials were fast and lasted few seconds; to reach the target force, the prosthesis had to be closed at a certain velocity and this determined the trial duration. For example, for $70 \%$ target force, the time from the start of the prosthesis closing to reaching a stable grasping force was less than $2 \mathrm{~s}$.

In the force steering test, the task was to control the force of an already closed prosthesis so that it tracked a 110-s long pseudorandom reference trajectory comprising a sequence of gradual, increasing and decreasing slopes. This time the subjects had to control manually both force increase and decrease using flexor and extensor muscles, respectively (no auto-open). The reference force level was indicated by the target force bar (Fig. 3, green line) moving according to the time profile of the reference trajectory, and the task for the subject was to produce the muscle activity generating the grasping force that would track the moving reference as close as possible (Fig. 3, semitransparent blue bar following the green target line). The subjects performed four tracking trials using simultaneous EMG and force feedback (EMG/FORCE, novel approach) and force feedback only (FORCE, classic approach). The first trial was regarded as a warming up and was not used for the data analysis. In both routine grasping and force tracking, the order of the feedback conditions was randomized between the subjects.

\section{Data analysis}

The variability of the generated forces was expressed as interquartile range (IQR) and used to evaluate the consistency in the control of force (i.e., precision). The accuracy was assessed by computing the absolute error defined as the absolute value of the difference between the generated and desired grasping force. Bartlett multiple-sample test for equal variances was applied to determine statistically significant difference in dispersions within the conditions overall, followed by AnsariBradley two-sample test with Bonferroni correction for pairwise comparisons of the force variability between the conditions. The quality of force tracking was assessed by calculating the root mean square tracking error (RMSE) between the generated and reference force profiles. All the results were reported as normalized forces, either in fractions or percent, i.e., 1 or $100 \%$ corresponded to the maximum force of the prosthesis $(\sim 100 \mathrm{~N})$. The stability of force control during force steering was assessed by determining the number of sudden drops in force. A drop was detected if the force fell below $10 \%$ over those segments of the reference trajectory where the reference force was $20 \%$ and higher. The statistically significant difference in absolute errors during the routine grasping and in RMSE during the force tracking between the two feedback conditions were evaluated using Wilcoxon signed rank test, as the data did not pass the normality test (one sample Kolmogorov-Smirnov). The threshold for the statistical significance was adopted at $p<0.05$.

\section{Results}

\section{Routine grasping}

Figure 4 shows a representative result from an ablebodied subject performing the routine grasping task in two feedback conditions (EMG/FORCE and FORCE) and with three levels of target force (30\%, $50 \%$ and $70 \%)$. When the EMG biofeedback was provided (Fig. 4[a]), the generated forces were stable and consistent across trials, i.e., the points closely concentrated around the corresponding reference force levels. The lines connecting the points were parallel and well separated. With the force feedback only (Fig. 4[b]), the generated forces were more variable across trials. The connecting lines deviated from the reference, sometimes closely approaching the (wrong) neighboring force level. In addition, the subjects spent few initial trials $(<10)$ tuning the prosthesis control in order to reach the desired force. The initial contractions for 50 and $70 \%$ target were too low and the subject gradually increased the strength, through several trials, before finally arriving into the vicinity of the desired force. When the EMG biofeedback was provided, there was no need for this iterative adjustment, i.e., the subjects used the feedback to adjust the muscle contraction, generating the myoelectric signal that was close to the reference, and thereby producing the desired level of force already in the first trial (zero warmup).

Summary results for the able-bodied subjects and all conditions are presented in Fig. 5(a). Providing the EMG biofeedback significantly improved the consistency in generating the grasping forces at all three force levels. Without the EMG biofeedback, the IQR was $10 \%, 14 \%$ and $16 \%$ for the target force of $30 \%, 50 \%$ and $70 \%$, respectively, and it was approximately twofold lower when the EMG biofeedback was transmitted (i.e., $6 \%, 6 \%$ and $7 \%$, respectively). With the force feedback only, the force variability increased significantly for the higher target forces (FORCE (30 \%) vs. FORCE (50 \%) and FORCE (70 \%) in Fig. 5[a]), which is a known trend [11]. When the EMG biofeedback was present, however, the dispersion was 


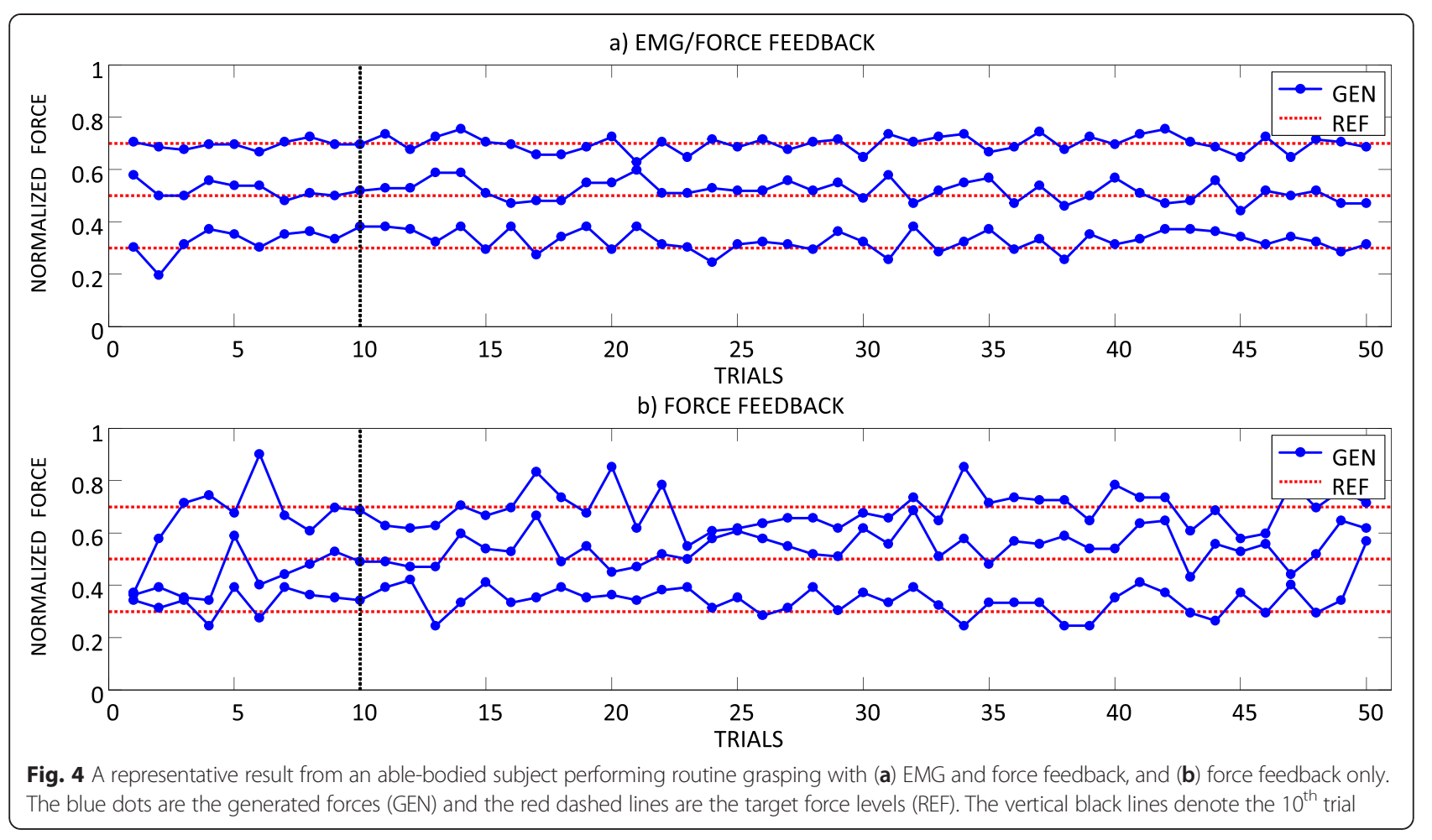

similar across all target force levels (no statistically significant differences). The occasional outliers in the generated forces, characteristic for the routine grasping using myocontrol [11], were less far from the median force when the EMG biofeedback was provided. Finally, the absolute errors (mean \pm standard deviation) from the desired forces were twice smaller with the EMG biofeedback ( $5 \pm 4 \%$ vs. $10 \pm 8 \%)$, and this difference was statistically significant $(p<0.001)$.

The results for the two amputee subjects are shown in Fig. 5(b), demonstrating the similar trend as in ablebodied subjects. The provision of the EMG biofeedback reduced the IQR of the generated forces from $13 \%, 9 \%$ and $16 \%$ for FORCE to $9 \%, 8 \%$, and $10 \%$ for EMG/ FORCE for the target forces of $30 \%, 50 \%$, and $70 \%$, respectively. The relative improvement was however less than in able-bodied subjects. Likewise, the amputee subjects were more accurate in generating the target forces with EMG biofeedback, which reduced the absolute errors (mean \pm standard deviation) from $11 \pm 10 \%$ for FORCE to $6 \pm 6 \%$ for EMG/FORCE.

\section{Force steering}

The representative trials of force tracking recorded from an able-bodied subject in two feedback conditions are depicted in Fig. 6. In both cases, the generated force increased/decreased in sharp, discrete steps. This discontinuous modulation of force is an inherent characteristic of the prosthesis operation, related to e.g. intrinsic friction effects. However, with the EMG biofeedback, the steps were smaller in magnitude, and the generated force trajectory resembled the reference profile, although the resolution of the generated profile was coarser. With the force feedback only, the control of the force increment/decrement magnitudes was rather poor, and the generated trajectory oscillated around the reference with large under and overshoots. The overall profile of the reference was poorly represented in the generated trajectory. Several times, especially during the decreasing segments, the force dropped suddenly to zero. Summary results for the quality of tracking over all able-bodied subjects are given in Fig. 7(a) and (b). Providing the EMG biofeedback reduced the tracking errors. The decrease was modest but statistically significant $(15.5 \pm 2 \%$ for FORCE vs. $13.5 \pm 2 \%$ for EMG/FORCE, $p<0.001)$. Similarly, the presence of EMG biofeedback improved the stability of tracking, since the number of force drops decreased from $10 \pm 4$ for FORCE to $7 \pm 3$ for EMG/FORCE $(p<0.001)$.

The results for the quality of tracking in amputee subjects are presented in Fig. 7(c) and (d). The outcome measures were better when EMG biofeedback was provided. The tracking errors decreased from $16.8 \%$ and $18.8 \%$ in FORCE to $13 \%$ and $17.8 \%$ in EMG/FORCE for the amputee 1 and 2, respectively. The number of drops in amputee subjects was higher compared to able-bodied, and it also decreased when using EMG biofeedback, from 18 and 24 in FORCE to 14 and 11 in EMG/FORCE for amputee 1 and 2 , respectively. 


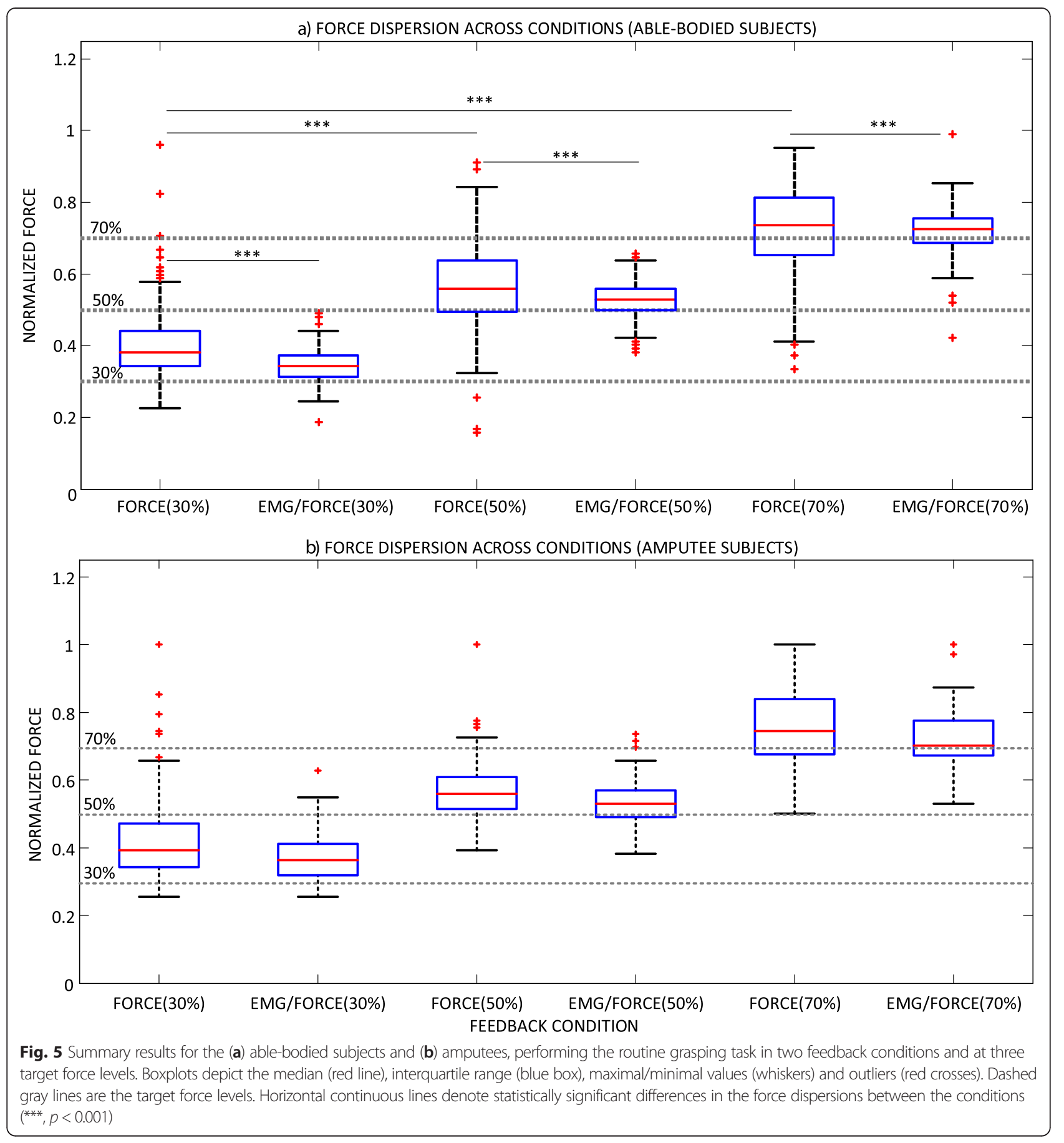

\section{Discussion}

A novel concept for closing the loop in myoelectric prostheses was demonstrated. In addition to feeding back the system output (generated grasping force), which is the classic method [9], in the novel approach the system input (myoelectric control signal) was also transmitted back to the user. The tests demonstrated that the provision of the EMG biofeedback improved the performance in both routine grasping and force tracking tasks. In the routine grasping, the online information about the prosthesis input allowed the subjects to adjust the motor command during the closing of the prosthesis so that the desired level of grasping force was achieved when the object was contacted. The subjects employed this simple predictive control scheme to anticipate the resulting grasping force. The EMG biofeedback also assisted the modulation of force while the prosthesis was closed (force tracking). During this task, the biofeedback 


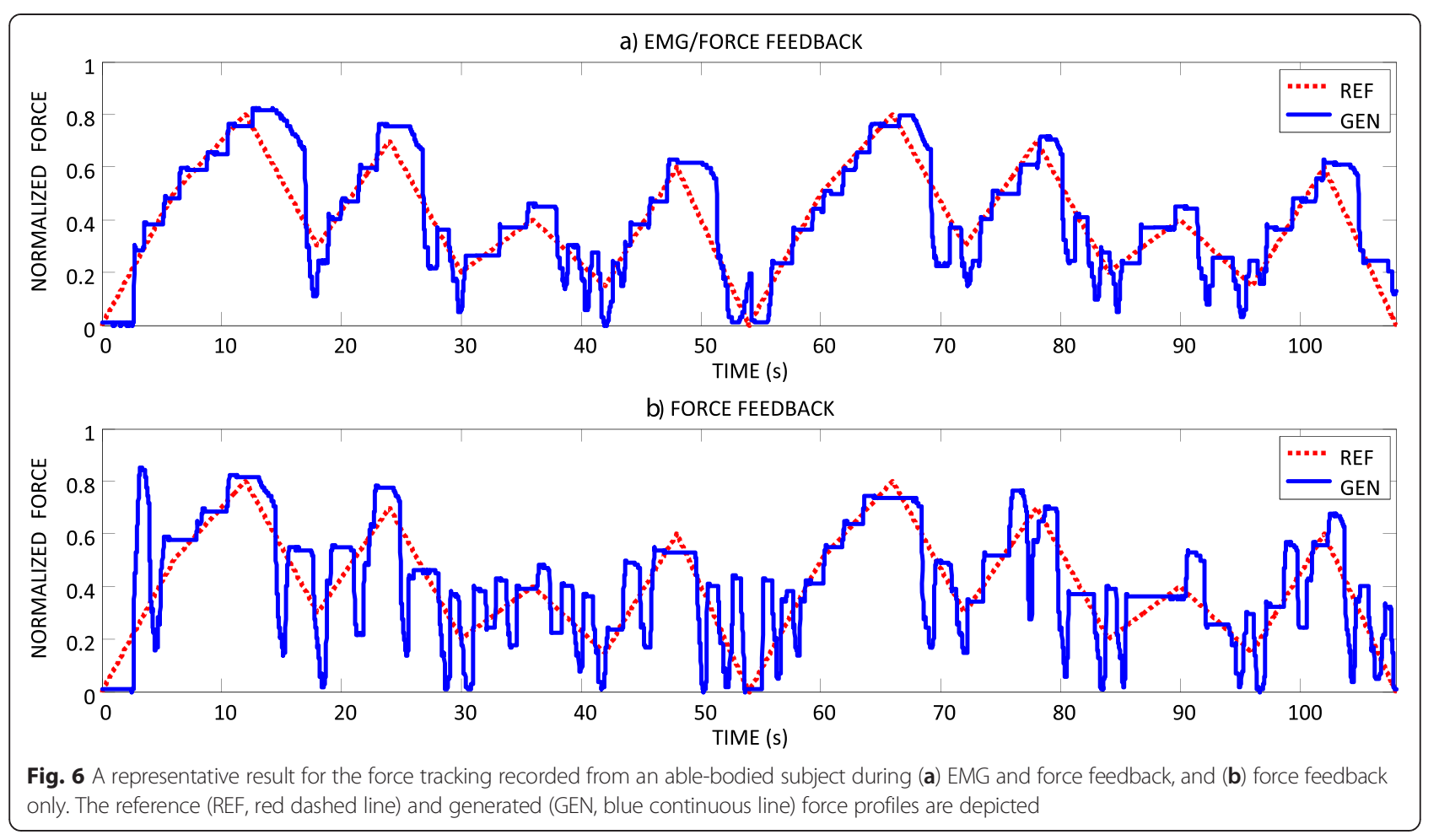

allowed the subjects to monitor the ongoing myoelectric activity and compare it to the current thresholds for the prosthesis activation (force increase/decrease). With this, they were able to finely regulate the myoelectric signals around the respective threshold levels and thereby control the timing as well as the magnitude of the force increase/decrease, improving the effective resolution of the generated force trajectory as well as the stability of tracking (fewer force drops). The statistically significant but overall modest decrease in the RMSE reflects the inherent limitations of the force modulation mechanism in the prosthesis (force jumps) as well as the nature of the task (continuous force tracking). The reference force trajectory was such that the subjects gradually modulated the strength of the muscle contraction. The advantage of the EMG biofeedback might be even better expressed during a step force regulation: grasp an object with a certain force, relax muscles (prosthesis maintains the force), and then increase/decrease the force to a higher/ lower force level. Importantly, the tests in two amputee subjects demonstrated that the EMG biofeedback can improve the performance even in experienced users of myoelectric prostheses. This is a preliminary but optimistic result that will be further evaluated in a future study including a larger pool of amputee subjects.

EMG biofeedback has been extensively used in the past in many fields of application, including rehabilitation, but the context was different [16]. For example, it is used during the user training to explain the principle of operation of the myoelectric prosthesis (e.g., as a didactic instrument) [17]. To our knowledge, this study is the first demonstration that the subjects can employ this type of information to improve the online control of the prosthesis grasping forces. The envisioned goal is to integrate this feedback as a standard component to enhance a daily-life prosthesis application. For the latter, the EMG biofeedback would have to be delivered through a tactile interface, as discussed later. Another possibility would be to implement the same protocol as in the current study by using a wearable augmented reality module (e.g., Google Glass). The module could connect to the prosthesis directly via a Bluetooth link and the EMG biofeedback bars could be shown on the wearable displays in the peripheral vision field. This was however outside the scope of the current proof-of-concept study. Nevertheless, even the current setup, with a host PC and the EMG biofeedback delivered on the computer screen, could be used as an instrument for the functional prosthesis training. It could assist the subjects in learning consistent force control, since it explicitly depicts the predictive mapping between the myoelectric command and the resulting grasping force. In addition, the EMG biofeedback could be utilized in daily life (electrotactile, augmented reality) or in the lab (host PC setup) to train the subjects to better exploit the natural proprioceptive feedback coming from their own muscles for the closedloop prosthesis control. By controlling the prosthesis while assisted through the EMG biofeedback, the subjects could learn the mapping between the sensation of muscle 


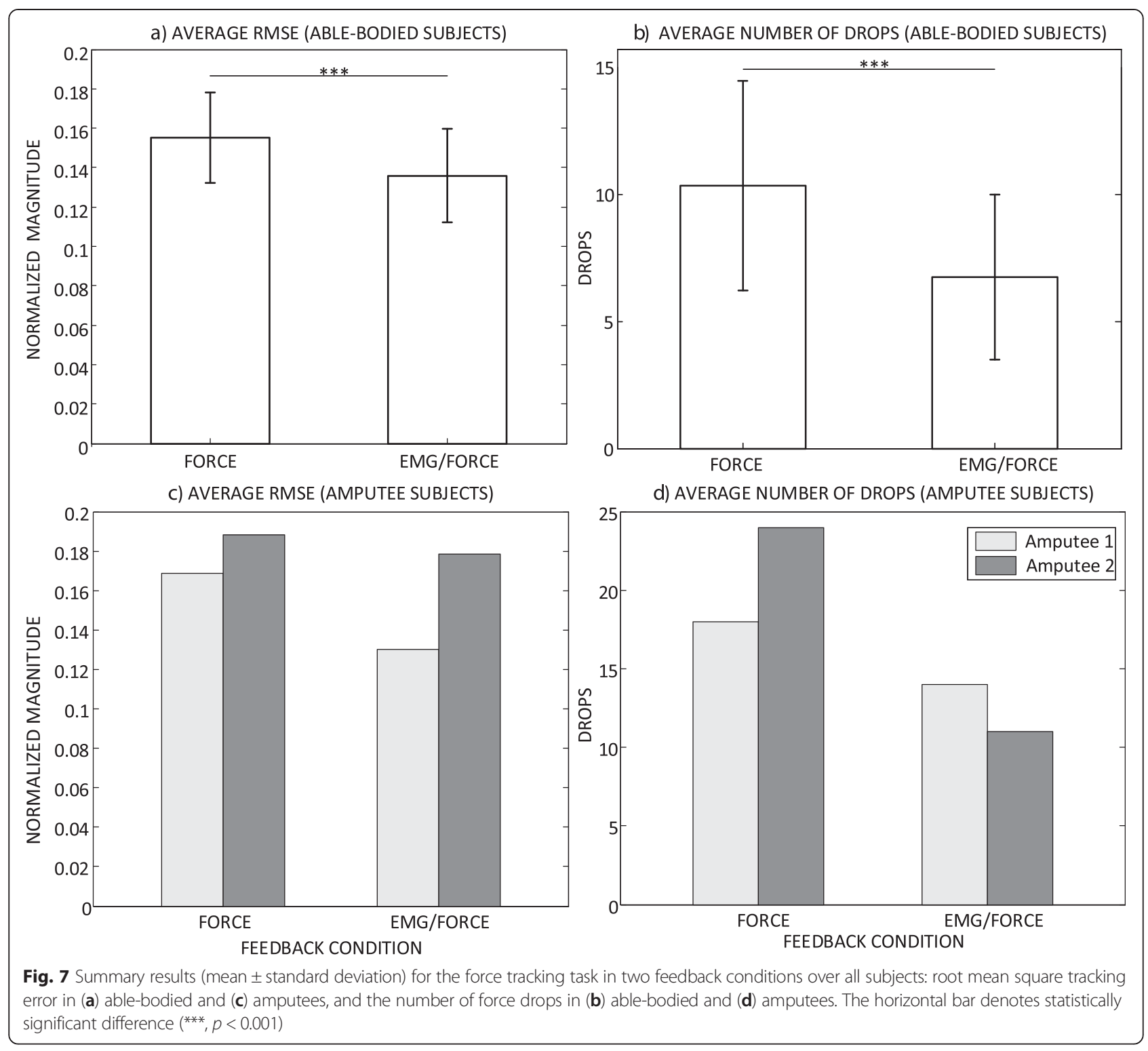

contraction, including the sense of effort, and the resulting grasping force. After some time, this mapping could stabilize and even render the EMG biofeedback redundant. To investigate this possibility, a future study will include a multi-session biofeedback protocol. In that sense, it would be especially relevant to test this training in the subjects that are experienced in myoelectric control. These subjects might have already learned to utilize the muscle proprioceptive feedback for control and the EMG biofeedback might not improve the performance substantially. However, the preliminary tests in the present study as well as the results in [11] point out that this might not be the case.

The presented approach can be related to a model of the biological motor control $[18,19]$. It is hypothesized that humans acquire internal models of the body dynamics and use them to control the movements in a predictive manner.
By applying the motor commands to the forward models, the system can be simulated to predict the expected sensory consequences of the movement (reafference). The estimated reafference can then be used for the closed-loop control, compensating for the delays that are inherent to the "conventional" sensory feedback transmitted through the peripheral neural pathways. In essence, the EMG biofeedback can be regarded as a simple feedforward simulation of a linearized prosthesis. It provides the subject with an estimate (prediction) of the grasping force, which will be developed when the hand contacts the object. This allows the subject to adjust the current online command (reafference-based control) even before the force begins developing (control based on the online sensory feedback).

In our previous work [11], we demonstrated that the velocity of prosthesis closing can be used for a predictive 
control of grasping force. In the present study, the subjects had access to this information indirectly, since they had a clear view on the virtual gripper. Yet, the EMG biofeedback still improved the performance of force control. One more possibility would be to provide the closing velocity explicitly, using a visual bar (as for the EMG). However, implementing the predictive force control using EMG rather than velocity has several advantages. First, the feedback on velocity belongs to a classic scheme, in which the system state is transmitted to the user. Therefore, the system dynamics is still in the loop, i.e., the modulation of velocity is limited by the system responsiveness to user commands, including both mechanical (e.g., inertia) and computational (e.g., command processing and implementation) factors. On the other side, the modulation of EMG is virtually instantaneous. Second, feedback on velocity is meaningless after contact, since the velocity becomes zero. Therefore, it cannot be used to assist force steering. Thirdly, the EMG biofeedback can be implemented using standard prosthesis components, while to transmit the velocity one needs a velocity sensor (gyroscope) or a position sensor, where the latter has to provide a signal good enough to allow differentiation (which is not the case in Michelangelo Hand).

The aim of the current study was to describe the approach and test the concept feasibility. Therefore, the feedback was provided using an ideal interface (visual bar). The same approach could be implemented using electrotactile stimulation by transmitting the information about the magnitude of the control signal through a single-channel intensity and/or frequency and/or multichannel spatial modulation. In the latter case, multiple stimulation electrodes can be used to implement an electrotactile equivalent of the visual bar, i.e., each electrode is associated to a signal range, and the current level of EMG is communicated by the currently active electrode within the array. Since the prosthesis is linearized, this also indicates the corresponding level of grasping force, once the prosthesis contacts the object. In order to produce a certain grasping force, the subject needs to activate the muscles so that a desired electrode starts stimulating. Providing the EMG biofeedback in this manner could result in a self-contained prosthetic system with an improved consistency of force control. The users would be able to produce a desired level of force repeatedly and reliably, eliminating the baseline variability as well as sudden large outliers that are characteristic for classic myocontrol [11]. Implementing the electrotactile EMG biofeedback to test these hypotheses is the work in progress.

This is not however a simple task since there a number of questions still to be answered. Ideally, two variables (EMG and force) need to be communicated to the user. This can be accomplished by using separate interfaces (dedicated electrodes) or the same interface with separate coding (see the video EMGBiofeedback.wmv and accompanying explanation in the Additional file 1). In any case, this adds an additional complexity to the system and also for the user, regarding his/her ability to perceive and utilize this information. In principle, however, the system can be simplified by implementing only the EMG biofeedback. Leaving out the force feedback would not affect the performance during routine grasping and the upward force steering, since in these cases the force corresponds to the level of EMG (linearized prosthesis). For the downwards force steering, the feedback would not communicate the current force level (force feedback), but the user would still be able to control the force transitions (EMG biofeedback). In any case, substituting the visual with a tactile interface, certainly decreases the quality of the information transfer. Pure spatial coding, for example, is intuitive for the subject to understand, but also limited to transmitting a set of discrete levels (each electrode one level). Mixed coding can increase the resolution but also the user cognitive effort. There are also limitations due to the technologies, such as, narrow dynamic range in electrostimulation due to discomfort at the higher stimulation intensities. All in all, it is still to be investigated how these factors (e.g., decrease in resolution, cognitive efforts) would affect the hereby demonstrated advantages of the EMG biofeedback as well as the overall user experience and acceptance of this approach.

Importantly, there are also limitations that must be considered when applying this approach in amputees. In the present study, the quality of myoelectric interfacing was improved by applying abrasive gel. In the real-life application, this is not available as only normal gel is used to moisturize the skin. Also, the quality of the myoelectric signals will depend on the condition of the residual limb (e.g., weaker muscles, scar tissue). This can compromise the myoelectric control in both cases, with classical force and EMG biofeedback. The impact of these factors and possible mitigation strategies have to be tested in the future work.

The consistency and accuracy of grasping reflect how reliable the system is in reproducing the user intention to grasp an object with a specific force, repeatedly and routinely. This is relevant for utility but also embodiment. Human hand is a reliable end effector, which responds promptly and consistently to user intentions, and if the artificial substitute would have similar characteristics, this would promote the effective substitution, both functionally and psychologically. In addition to improving the repeated grasping with the same force, the EMG biofeedback could also facilitate switching between forces across trials, as explained in the previous paragraphs. From the functional viewpoint, the provision of feedback makes the task demands explicit, i.e., the user can establish a mapping 
between daily life tasks and the grasping forces that are necessary to perform those tasks. If the user is also confident that he/she can generate those forces accurately and consistently, this could facilitate the optimal utilization of the prosthesis (economical grasping paradigm [20]). For example, if the EMG biofeedback is implemented using electrotactile stimulation with spatial coding at $\mathrm{N}$ levels, the user would know that he/she can generate $\mathrm{N}$ levels of force reliably. Through the use of the prosthesis, he/she would learn that specific tasks can be accomplished using certain forces, e.g., to pick grapes without squeezing them the force should be set at the level 2. Therefore, the user would determine the target force based on experience, and then generate that force fast and reliably using the EMG biofeedback interface.

The quality of force steering assessed through RMSE is relevant for object holding and manipulation. For example, when the force is gradually applied to a delicate object (e.g., wine glass) or when the force needs to be gradually decreased, e.g., for a smooth passing of an object from the prosthesis to a contralateral hand or to another person. In practice, unilateral amputees accomplish such sensitive tasks most often using a healthy hand, due to a poor controllability and other limitations [21]. A system that would improve the force modulation could increase the applicability of the prosthesis, and therefore improve the tradeoff between the efforts (training, mounting, maintenance) and gained functionality.

In the present experiment, some of the feedback cues that would normally be available to the prostheses users have been blocked. For example, most of the present day prostheses, including Michelangelo Hand, produce noise during movement and force modulation. However, it is unlikely that these additional feedback sources would affect the results and conclusions of the present study. Those cues indicate the prosthesis state (aperture and force), which was anyway clearly disclosed to the subjects using visual feedback (virtual gripper and force bar). Due to this and the phenomenon of visual dominance [22], it is unlikely that the additional cues, such as sound, would significantly improve the state assessment and therefore affect the overall performance. However, in a real-life application when the feedback is communicated through a practical electrotactile and/or vibrotactile interface and a visual assessment is non-ideal (e.g., viewing angle, occlusions), the incidental feedback could be more relevant. Importantly, this would mainly affect the force control using classic force feedback. From that point of view, the EMG biofeedback is rather robust, since the myoelectric command is adjusted based on the feedback about the state of the user (and not that of the prosthesis).

Myoelectric control can also be improved by applying specialized processing to the surface signals [23] and/or acquiring better signals through implanted interfaces [24].
Both approaches can substantially improve the stability and precision of the myoelectric waveforms. Importantly, these developments do not rule out the usefulness of the EMG biofeedback. More consistent signals lead to more consistent control, but the mapping between the subjective sense of muscle contraction and the resulting grasping force would still remain elusive. The latter connection can be made explicit by providing the EMG biofeedback to the user.

In this study, we have used a state of the art myoelectric hand, the latest model from Otto Bock. Importantly, the obtained insights and conclusions are general, since most myoelectric prostheses share the same principle of operation. Furthermore, the EMG biofeedback is not specific to force control. It could be utilized in a similar manner to facilitate the control of other prosthesis variables/degrees-of-freedom (e.g., velocity of opening/ closing, velocity of wrist rotation).

\section{Conclusions}

The present study proposes a novel paradigm to close the loop in a myoelectric prosthesis. In the classic approach, the feedback transmits to the user the state of the prosthesis (aperture, velocity and/or force), whereas in the novel method the feedback also informs the user about his/her own latent variables, i.e., the myoelectric signals he/she generates (EMG biofeedback). The experiments demonstrated that the provision of the EMG biofeedback improved the quality of force control both in routine grasping and force steering tasks, and both in able-bodied subjects and two amputees who were experienced users of myoelectric prostheses. With the EMG biofeedback displayed as a visual bar on the computer screen, the subjects could see and modulate the current level of their muscle activity, and thereby explicitly control the command they send to the prosthesis. In the conventional approach, the myoelectric signals are latent variables, which can be controlled only by using indirect cues, such as subjective experience (sensation of muscle contraction) and/or observable consequences (e.g., prosthesis movement). These sources are however unreliable, especially due to the inherent variability of the myoelectric signals recorded using surface electrodes. EMG biofeedback allows the user to improve the precision and accuracy of myoelectric commands using active control, i.e., fast local loop in which the user modulates the strength of muscle contraction based on the online EMG biofeedback. The present study demonstrated the feasibility, and the next step is the implementation of this approach using practical interfaces, such as electrotactile stimulation and augmented reality glasses, and the validation in a larger pool of subjects. Therefore, there are many practical questions still to address (e.g., functional gain vs. user efforts vs. acceptability), but the present results are very optimistic. The prosthesis 
equipped with the EMG biofeedback might increase the user confidence in the system, by allowing consistent and reliable force control, and this can improve the utility, embodiment and ultimately the acceptance rate. Furthermore, the EMG biofeedback could be also considered as a temporary add-on to the prosthesis, an instrument for training the subject to exploit the natural feedback from his/her own muscles for the closed-loop prosthesis control.

\section{Additional file}

\section{Additional file 1: The file contains a short movie}

(EMGBiofeedback.wmv) showing an amputee subject modulating the force of a prosthesis while holding an object. The force feedback and EMG biofeedback were implemented using electrotactile stimulation. The movie is explained in more detail in the accompanying text file (EMGBiofeedback.doc).

\section{Abbreviations}

EMG: Electromyography; MVC: Maximum voluntary contraction;

IQR: Inter-quartile range; RMSE: Root mean square error.

\section{Competing interests}

MM and BG are employed by Otto Bock HealthCare Gmbh, which is a company that produced the prosthesis used in the study (Michelangelo Hand).

\section{Authors' contributions}

SD, MM, BG, DF conceptualized the study. MM and KF conducted the experiments. SD, MM and KF analyzed the data. All authors participated in writing the manuscript. All authors read and approved the final manuscript.

\section{Acknowledgment}

This work is financially supported by the German Ministry for Education and Research (BMBF) via the Bernstein Focus Neurotechnology (BFNT) Göttingen under the grants No. $01 \mathrm{GQ0817}$ and 01GQ0810, and the European Commission under the MYOSENS (FP7-PEOPLE-2011-IAPP-286208) projects.

\section{Author details}

'Department of Neurorehabilitation Engineering, University Medical Center Göttingen (UMG), Georg-August University, 37075 Göttingen, Germany. ${ }^{2}$ Otto Bock HealthCare GmbH, 37115 Duderstadt, Germany.

Received: 18 December 2014 Accepted: 2 June 2015

Published online: 19 June 2015

\section{References}

1. MacKenzie C, Iberall T. The Grasping Hand. Amsterdam: Elsevier B.V; 2010.

2. Parker $P$, Englehart $K$, Hudgins B. Myoelectric signal processing for control of powered limb prostheses. J Electromyogr Kinesiol. 2006;16(6):541-8.

3. Asghari Oskoei M, Hu H. Myoelectric control systems-A survey. Biomed Signal Process Control. 2007;2(4):275-94.

4. "Vincent Evolution 2 Hand." [Online]. Available: http://vincentsystems.de/en/ prosthetics/vincent-evolution-2/. [Accessed: 09-Oct-2014].

5. Raspopovic S, Capogrosso M, Petrini FM, Bonizzato M, Rigosa J, Di Pino G, et al. "Restoring natural sensory feedback in real-time bidirectional hand prostheses.,". Sci Transl Med. 2014;6:222. ra19.

6. Szeto AY, Saunders FA. Electrocutaneous stimulation for sensory communication in rehabilitation engineering. IEEE Trans Biomed Eng. 1982;29(4):300-8.

7. Kaczmarek KA, Webster JG, Bach-y-Rita P, Tompkins WJ. "Electrotactile and vibrotactile displays for sensory substitution systems,". IEEE Trans Biomed Eng. 1991;38:1-16. no. 1. leee.

8. Peerdeman B, Boere D, Witteveen $H$, Huis in 'tVeld R, Hermens H, Stramigioli S, et al. "Myoelectric forearm prostheses: State of the art from a user-centered perspective,". J Rehabil Res Dev. 2011;48(6):719.
9. Antfolk C, D'Alonzo M, Rosén B, Lundborg G, Sebelius F, Cipriani C. Sensory feedback in upper limb prosthetics. Expert Rev Med Devices. 2013;10(1):45-54.

10. Clancy E, Morin E, Merletti R. Sampling, noise-reduction and amplitude estimation issues in surface electromyography. J Electromyogr Kinesiol. 2002;12(1):1-16.

11. Ninu A, Dosen S, Muceli S, Rattay F, Dietl H, Farina D. Closed loop control of grasping with a myoelectric hand prosthesis: which are the relevant feedback variables for force control? IEEE Trans Neural Syst Rehabil Eng. 2014;22(5):1041-52.

12. Biddiss EA, Chau TT. Upper limb prosthesis use and abandonment: a survey of the last 25 years. Prosthet Orthot Int. 2007;31(3):236-57.

13. Bouwsema $\mathrm{H}$, van der Sluis $\mathrm{CK}$, Bongers RM. "Effect of feedback during virtual training of grip force control with a myoelectric prosthesis.,". PLoS One. 2014;9(5):e98301.

14. Dosen S, Markovic M, Hartmann C, Farina D. Sensory feedback in prosthetics: a standardized test bench for closed-loop control. IEEE Trans Neural Syst Rehabil Eng. 2015;23(2):267-76.

15. "Otto Bock Michelangelo Hand," 2014. [Online]. Available: http://www.living-with-michelangelo.com/gb/home/.

16. Giggins OM, Persson UM, Caulfield B. Biofeedback in rehabilitation. J Neuroeng Rehabil. 2013;10:60.

17. "Myoboy training system." [Online]. Available: http//professionals. ottobockus.com/cps/rde/xbcr/ob_us_en/ifu_647g265_myoboy_757m11.pdf. [Accessed: 09-Oct-2014].

18. Franklin DW, Wolpert DM. Computational mechanisms of sensorimotor control. Neuron. 2011;72(3):425-42.

19. Wolpert DM, Miall RC. Forward models for physiological motor control. Neural Netw. 1996;9(8):1265-79.

20. Saunders I, Vijayakumar S. "The role of feed-forward and feedback processes for closed-loop prosthesis control.,". J Neuroeng Rehabil. 2011;8(1):60.

21. Østlie K, Lesjø IM, Franklin RJ, Garfelt B, Skjeldal OH, Magnus P. Prosthesis use in adult acquired major upper-limb amputees: patterns of wear, prosthetic skills and the actual use of prostheses in activities of daily life. Disabil Rehabil Assist Technol. 2012;7(6):479-93.

22. Hecht $\mathrm{D}$, Reiner M. Sensory dominance in combinations of audio, visual and haptic stimuli. Exp Brain Res. 2009;193(2):307-14.

23. Sanger TD. Bayesian filtering of myoelectric signals. J Neurophysiol. 2007;97(2):1839-45.

24. Pasquina PF, Evangelista M, Carvalho AJ, Lockhart J, Griffin S, Nanos G, et al. "First-in-man demonstration of a fully implanted myoelectric sensors system to control an advanced electromechanical prosthetic hand". J Neurosci Methods. 2015;244:85-93.

\section{Submit your next manuscript to BioMed Central and take full advantage of:}

- Convenient online submission

- Thorough peer review

- No space constraints or color figure charges

- Immediate publication on acceptance

- Inclusion in PubMed, CAS, Scopus and Google Scholar

- Research which is freely available for redistribution 



\section{Curriculum Vitae - Marković Marko}

PERSONAL DATA

Early Stage Researcher

Translational Research and Knowledge

Management

Ottobock HealthCare GmbH

37115 Duderstadt

004917671911745

April 30, 1987

Belgrade

Serbia

marko.markovic@ottobock.de

\section{RESEARCH INTERESTS / RESEARCH PROFILE}

Main interests: Closed loop upper limb prosthesis control, Sensor-fusion algorithms, Computer-vision, Augmented reality

Secondary interests: System development in MATLAB Simulink environment

\section{EDUCATION}

Enrolled in PhD program „Humanwissenschaften in der Medizin“ at University of Göttingen, 2014

Master of Engineering, University of Belgrade, 2011, Biomedical Engineering (GPA 10 out of 10)

Bachelor of Engineering, University of Belgrade, 2010, Biomedical Engineering (GPA 8.78 out of 10)

\section{PROFESSIONAL EXPERIENCE}

Ottobock HealthCare GmbH, Germany, 2014 - 2016, Early stage researcher in European MYOSENS project

University of Göttingen, Germany, 2012-2014, Research Assistant at Department of Neurorehabilitation Engineering, European DEMOVE project

Institute of Physics Belgrade, Serbia, 2011-2012, Research Assistant, European ATLAS-CERN collaboration project 


\section{PUBLICATIONS}

\section{$\underline{\text { Refereed Journal Publications }}$}

[1] M. Markovic, S. Dosen, C. Cipriani, D. Popovic, and D. Farina, "Stereovision and augmented reality for closed-loop control of grasping in hand prostheses.," J. Neural Eng., vol. 11, no. 4, p. 046001, Aug. 2014.

[2] Š. Matija, S. Kočović, M. Marković, and D. B. Popović, "Microsoft KinectBased Artificial Perception System for Control of Functional Electrical Stimulation Assisted Grasping," Biomed Res. Int., vol. 2014, p. 12, 2014.

[3] S. Dosen, M. Markovic, C. Hartmann, and D. Farina, "Sensory feedback in prosthetics: a standardized test bench for closed-loop control.," IEEE Trans. Neural Syst. Rehabil. Eng., vol. 23, no. 2, pp. 267-76, Mar. 2015.

[4] S. Dosen, M. Markovic, N. Wille, M. Henkel, M. Koppe, A. Ninu, C. Frömmel, and D. Farina, "Building an internal model of a myoelectric prosthesis via closed-loop control for consistent and routine grasping.," Exp. brain Res., vol. 233, no. 6, pp. 1855-65, Jun. 2015.

[5] S. Dosen, M. Markovic, S. Kelef, B. Graimann, and D. Farina, "EMG Biofeedback for online predictive control of grasping force in a myoelectric prosthesis," J. Neuroeng. Rehabil., vol. 12, no. 55, pp. 1-13, 2015.

[6] M. Markovic, S. Dosen, D. Popovic, B. Graimann, and D. Farina, "Sensor fusion and computer vision for context-aware control of a multi degree-offreedom prosthesis.," J. Neural Eng., vol. 12, no. 6, p. 066022, Nov. 2015

[7] M. Schweisfurth, M. Markovic, S. Dosen, F. Teich, and D. Farina, "Electrotactile EMG feedback improves the control of prosthesis grasping force," J. Neural Eng., 2015, in review.

\section{Conference and Workshop Proceedings (Reviewed)}

[1] M. Štrbac and M. Marković, "Stereovision system for estimation of the grasp type for electrotherapy," Serbian J. Electr. Eng., vol. 8, no. 1, pp. 17-25, 2010.

[2] M. Canela, A. J. Ama, and J. L. Pons, Converging Clinical and Engineering Research on Neurorehabilitation, vol. 1. Berlin, Heidelberg: Springer Berlin Heidelberg, pp. 277-281, 2013.

[3] C. Castellini, P. Artemiadis, M. Wininger, A. Ajoudani, and M. Alimusaj, "Proceedings of the first workshop on Peripheral Machine Interfaces : going beyond traditional surface electromyography," Front. Neurorobot., 2014

[4] M. Marković, B. Graimann, S. Došen, and D. Farina, "Computer vision for automatic control of orientation and preshape in a hand prosthesis with an active wrist," in MEC 2014, pp. 143-145, 2014. 


\section{PATENTS}

[1] S. Dosen, M. Markovic, D. B. Popović, D. Farina, and B. Graimann, "Sensor Fusion for Control of Upper Limb Protheses: Integration of Myoelectric Control with Stereovision, Augmented Reality and Inertial Sensing," EP 13171671.4, 2013.

20.01.2016 
